Draft V. 2011 JuLY 27

Preprint typeset using LATEX style emulateapj v. 5/2/11

\title{
THE STAR FORMATION REFERENCE SURVEY. I. SURVEY DESCRIPTION AND BASIC DATA
}

\author{
M. L. N. Ashby ${ }^{1}$, S. Mahajan ${ }^{1,2}$, H. A. Smith ${ }^{1}$, S. P. Willnner ${ }^{1}$, G. G. Fazio ${ }^{1}$, S. Raychaudhury ${ }^{2}$, A. Zezas $^{1,3}$, \\ P. Barmby ${ }^{4}$, P. Bonfini ${ }^{3}$, C. CaO ${ }^{5}$, E. González-Alfonso ${ }^{6}$, D. Ishihara ${ }^{7}$, H. Kaneda ${ }^{7}$, V. Lyttle $^{4}$, S. Madden $^{8}, \mathrm{C}^{2}$

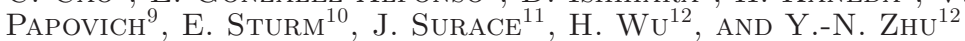 \\ Draft v. 2011 July 27
}

\begin{abstract}
Star formation is arguably the most important physical process in the cosmos. It is a fundamental driver of galaxy evolution and the ultimate source of most of the energy emitted by galaxies. A correct interpretation of star formation rate (SFR) measures is therefore essential to our understanding of galaxy formation and evolution. Unfortunately, however, no single SFR estimator is universally available or even applicable in all circumstances: the numerous galaxies found in deep surveys are often too faint (or too distant) to yield significant detections with most standard SFR measures, and until now there have been no global, multi-band observations of nearby galaxies that span all the conditions under which star-formation is taking place. To address this need in a systematic way, we have undertaken a multi-band survey of all types of star-forming galaxies in the local Universe. This project, the Star Formation Reference Survey (SFRS), is based on a statistically valid sample of 369 nearby galaxies that span all existing combinations of dust temperature, SFR, and specific SFR. Furthermore, because the SFRS is blind with respect to AGN fraction and environment it serves as a means to assess the influence of these factors on SFR. Our panchromatic global flux measurements (including GALEX FUV+NUV, SDSS ugriz, 2MASS $J H K_{s}$, Spitzer 3-8 $\mu \mathrm{m}$, and others) furnish uniform SFR measures and the context in which their reliability can be assessed. This paper describes the SFRS survey strategy, defines the sample, and presents the multi-band photometry collected to date.
\end{abstract}

Subject headings: Infrared: galaxies; — catalogs — stars: formation

\section{INTRODUCTION}

Star formation has been the most important single physical process since recombination. Not only have stars created most of the luminous energy in the Universe, they have produced the heavy elements needed for planets and life. Star formation has naturally been the subject of vast numbers of studies, leading, for exam-

\footnotetext{
${ }^{1}$ Harvard-Smithsonian Center for Astrophysics, 60 Garden St., Cambridge, MA 02138, USA [e-mail: mashby@cfa.harvard.edu]

${ }^{2}$ School of Physics and Astronomy, University of Birmingham, Edgbaston, Birmingham B15 2TT, UK

${ }^{3}$ University of Crete, Physics Department \& Institute of Theoretical and Computational Physics, 71003 Heraklion, Crete, Greece

${ }^{4}$ Department of Physics \& Astronomy, University of Western Ontario, London, ON N6A 3K7, Canada

${ }^{5}$ School of Space Science and Physics, Shandong University at Weihai, Weihai, Shandong 264209, China

${ }^{6}$ Universidad de Alcalá de Henares, Deptamento de Física, Campus Universitario, E-28871 Alcalá de Henares, Madrid, Spain

${ }^{7}$ Graduate School of Science, Nagoya University, Furo-cho, Chikusa-ku, Nagoya, Aichi 464-8602, Japan

8 Service d'Astrophysique, CEA/Saclay, l'Orme des Merisiers, 91191 Gif-sur-Yvette, France

${ }^{9}$ Department of Physics, Texas A \& M University, College Station, TX 77843-4242

${ }^{10}$ Max-Planck-Institute for Extraterrestrial Physics (MPE), Gieenbachstrae 1, D-85748 Garching, Germany

11 Spitzer Science Center, MS 220-6, California Institute of Technology, Jet Propulsion Laboratory, Pasadena, CA 91125, USA

12 Key Laboratory of Optical Astronomy, National Astronomical Observatories, Chinese Academy of Sciences, 20A Datun Road, 100012 Beijing, China
}

ple, to our current understanding of the star formation history of the Universe (e.g., Lilly et al. 1996, Hopkins \& Beacom 2006; Madau et al. 1998) and the discovery of the Schmidt Law (Schmidt 1959; updated by Kennicutt et al. 1998) relating the SFR in galaxies to the gas surface density.

A general inadequacy of existing work is the lack of a self-consistent treatment of SFR across the full electromagnetic spectrum: ultraviolet (UV) continuum only samples the SFR in the absence of dust; $\mathrm{H} \alpha$ and [O II] measure ionizing photons coming from only the highmass $\left(\gtrsim 5 \mathrm{M}_{\odot}\right)$ population. Emission in the polycyclic aromatic hydrocarbon (PAH) bands is taken as a SFR measure (e.g., Madden et al. 2006; Wu et al. 2005) but may be unreliable for low-luminosity (or low-metallicity) galaxies (Hogg et al. 2005) or in the presence of a hard radiation field. Far-infrared dust re-radiation samples a broad range of star formation $\left(\sim 1-10 \mathrm{M}_{\odot}\right.$ for reasonable IMFs) but only becomes a precise SFR measure in the optically-thick limit (Kennicutt 1998). Nonthermal radio emission comes from Type II supernova remnants (e.g., Rieke et al. 1980) and therefore only samples higher-mass SF.

Star formation activity is by no means evenly distributed throughout galaxies. Instead, stars form within the densest regions of giant molecular clouds; this phenomenon manifests in the IRAS bands as far-infrared radiation when the (hot) young stellar objects deeply embedded in their natal clouds illuminate the surrounding interstellar material (e.g., Parker 1991), which then reradiates that energy at long wavelengths. In a detailed case study of Milky Way GMC complexes (e.g., the California 
Nebula) Lada, Lombardi, \& Alves (2010) demonstrated a remarkably tight correlation between SFR and the total mass of dense gas, lending further support to this view.

There is considerable evidence in the literature that our understanding of star formation depends on making consistent use of all available wavebands. For example, Kartaltepe et al. (2010) showed that without photometry at wavelengths longer than $100 \mu \mathrm{m}$, total farIR luminosities (and thus SFRs) are typically underestimated by 0.2 dex; in some cases the discrepancy can be much larger. This can be interpreted as an inability to adquately measure emission from a cold dust component (if present) when such far-IR data are lacking. These issues are now becoming better understood as a result of $A K A R I$ and Herschel programs that reach deeper into the far-IR than could IRAS, ISO, or Spitzer. The situation remains complex, however. In Herschel-selected galaxies, dust attenuation appears to strongly impact the UV detection fraction and the relationship between UV and IR SFR indicators (Buat et al. 2010). There are strong hints of systematic discrepancies between AKARI and IRAS photometry at $\sim 100 \mu \mathrm{m}$ (Jeong et al. 2007; Figs. 1 and 7 of Takeuchi et al 2010). These unresolved issues can in principle be addressed with thoughtful controls. But there are also systematic effects intrinsic to the galaxies themselves. For example, there is evidence that the specific SFR (sSFR, the SFR per unit stellar mass) depends on stellar mass (e.g., Sobral et al. 2011, Elbaz et al. 2011). For many years there have been suggestions that dust temperature was dependent on luminosity (e.g., Sanders et al. 2003). And there is as yet no systematic treatment of the contribution of the older, quiescent stellar components to the SFR tracers most commonly used.

To better understand the complexities of star formation, a comprehensive treatment examining the influence of all the major parameters - stellar mass, dust mass and temperature, and metallicity - on SFR indicators is needed. The importance and timeliness of this subject is attested by numerous recent efforts to grapple with the nuances of SFR estimation. Notable examples include the Spitzer Infrared Nearby Galaxies Sample (SINGS; Kennicutt et al. 2003; Calzetti et al. 2010), the Local Volume Legacy Survey (LVLS; Dale et al. 2009), the Herschel Reference Survey (HRS; Boselli et al. 2010), the Great Observatories All-sky LIRG Survey (GOALS; Armus et al. 2009), and the Multi-Wavelength Extreme Starburst Sample (MESS; Laag et al. 2010), among others. Each of these undertakings has been designed to attack specific aspects of the star-formation phenomenon, but each also has limitations that prevent it from providing a comprehensive picture of star formation in galaxies.

This paper presents the Star Formation Reference Survey (SFRS), a sample of nearby galaxies having a unique capacity to describe star formation under all conditions in which it occurs in the local Universe. Section 2 describes the selection criteria used to define the SFRS. Section 3 presents the SFRS datasets obtained to date. Section 4 shows how the SFRS complements the abovementioned projects, presents an estimate of AGN prevalence and the ramifications for far-infrared SFR measures, and briefly describes SFRS-related observing campaigns now in progress.

\section{SAMPLE SELECTION}

The complexities of the real Universe mean that an understanding of global galaxy properties will inherently be statistical in nature. Progress, even in the local Universe, requires uniform measurements of the properties of a well-chosen, sufficiently large number of galaxies that they sample star formation across the full range of galaxy properties. In other words, having the proper study sample is critical to the success of this undertaking.

The SFRS selection criteria were defined objectively to guarantee that the sample spans the full range of properties exhibited by star-forming galaxies in the local Universe. We began with the IRAS $60 \mu \mathrm{m}$ luminosity as an unbiased (but perhaps not always correct!) star formation tracer by virtue of the breadth and uniformity of IRAS coverage and because of the proximity of the $60 \mu \mathrm{m}$ band to the SED peak associated with star formation. Although the selection was based squarely on the $60 \mu \mathrm{m}$ flux, this should not be taken as an assertion that all issues of interpretation have been resolved. Some fraction of the $60 \mu \mathrm{m}$ luminosity, at least in some galaxies, must arise from dust illuminated by the older stellar population. Addressing this matter is one of the aims of the SFRS project and a primary motivation for the assembly of the datasets described in Sec. 3.

The parent sample for the present study is the $\mathrm{PSCz}$ catalog (Saunders et al. 2000), a full-sky database of 15,000 nearby star-forming galaxies brighter than $0.6 \mathrm{Jy}$ at $60 \mu \mathrm{m}$. Most PSCz objects are closer than $z=0.2$. The PSCz is not biased toward relatively rare ultraluminous objects (unlike the 1 Jy sample of Kim, Veilleux, $\&$ Sanders 2002) and is more representative than more restricted samples (e.g., the IRAS Bright Galaxy Sample; $F_{60}>5.24 \mathrm{Jy}$; Sanders et al. 2003). PSCz galaxy luminosities range from $L(60 \mu \mathrm{m})=10^{7}$ to $10^{12} \mathrm{~L}_{\odot}$.

Star-forming galaxies have a wide range of properties, but aside from absolute luminosity, the specific SFR and the color temperature of far infrared dust emission are arguably the most important. These parameters reflect the relative importance of present and past star formation and the densities of the regions where stars are forming. High color temperature is usually taken to indicate that dust grains are close to the newly-formed stars, i.e., it is a measure of active star formation. A low color temperature (high $F_{100} / F_{60}$ ratio), on the other hand, can be interpreted as reflective of a situation in which the ambient UV field from older stars in the galaxy disk is illuminating dust in the ISM. Figure 1 illustrates one way in which dust temperature and luminosity relate. Nearly all 'cool' galaxies (i.e., those with a far-IR flux density ratio $F_{100} / F_{60} \geq 2$ ) reside in the low-luminosity regime. The situation is different for 'warmer' $\left(F_{100} / F_{60}<2\right)$ galaxies, however - this population is roughly evenly divided between high- and low-luminosity sources. To capture this behavior in a study sample, it is not adequate to make a simple luminosity cut: one must account for the dust temperature or be at risk of under-representing the mode (warm or cool) at which star formation occurs for some luminosities.

Figure 1 also reveals a dichotomy in the relationship of sSFR and luminosity. While essentially all the low-sSFR galaxies are low-luminosity objects, those with high sSFRs are a mix, with both low- and high-luminosity galax- 

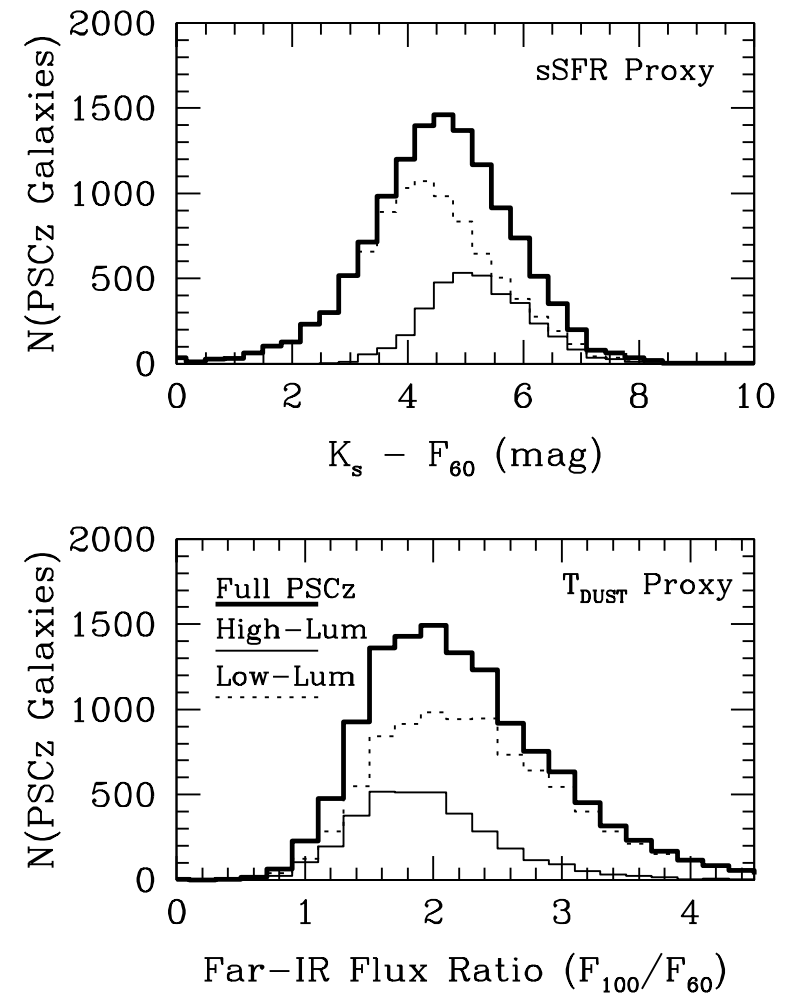

FIG. 1. - Histograms of sSFR and dust temperature proxies within the parent PSCz galaxy sample. Thick lines show galaxy numbers for the full sample. The PSCz has been divided into two subsamples at $\nu L_{\nu}(60 \mu \mathrm{m})=10^{9.5} \mathrm{~L}_{\odot}$. Thin solid lines indicate the distributions for the high-luminosity subsample, and dashed lines indicate the distributions for the low-luminosity subsample. Top panel: Abscissa is the ratio of $K_{s}$ to $60 \mu \mathrm{m}$ flux densities expressed as the difference in AB magnitudes. Bottom panel: Abscissa is the ratio of 100 to $60 \mu \mathrm{m}$ flux densities $\left(F_{\nu}\right)$.

TABLE 1

Bin Boundaries Applied to Parent PSCz Sample

\begin{tabular}{llll}
\hline \hline Parameter & Q1/Q2 & Q2/Q3 & Q3/Q4 \\
& & & \\
\hline sSFR Proxy $\left(K_{s}-F_{60}\right)$ & 3.78 & 4.56 & 5.34 \\
$T_{D U S T}$ Proxy $\left(F_{100} / F_{60}\right)$ & 1.71 & 2.14 & 2.69 \\
& & & \\
\hline NoTE. - The quartile boundaries applied to the sSFR \\
and $T_{D U S T}$ proxies used to define the SFRS sample. The \\
third selection dimension, $60 \mu$ m luminosity, was binned in \\
decades starting at $\log \left(\mathrm{L}_{60}\right)=10^{6.5} \mathrm{~L}_{\odot}$ with an additional \\
half-decade bin boundary at $10^{10} \mathrm{~L}_{\odot}$, near the peak of the \\
distribution (Fig. 2).
\end{tabular}

ies present. A selection on luminosity alone is therefore extremely unlikely to include sources with the full range of sSFRs that exist, and a high-luminosity selection will miss the low-sSFR sources entirely.

A full sampling of star-forming galaxy properties requires that the SFRS be defined in a three-dimensional space spanning the full ranges occupied by $\mathrm{PSC}$ galaxies in $60 \mu \mathrm{m}$ luminosity, flux ratio $F_{60} / K_{s}$, and far-IR flux density ratio $F_{100} / F_{60}$. In this scheme 2 MASS $K_{s}$ serves as a proxy for stellar mass, the far- to near-infrared flux ratio measures specific SFR, while the far-IR flux density ratio acts as a measure of dust temperature. This threedimensional parameter space was binned by decade in
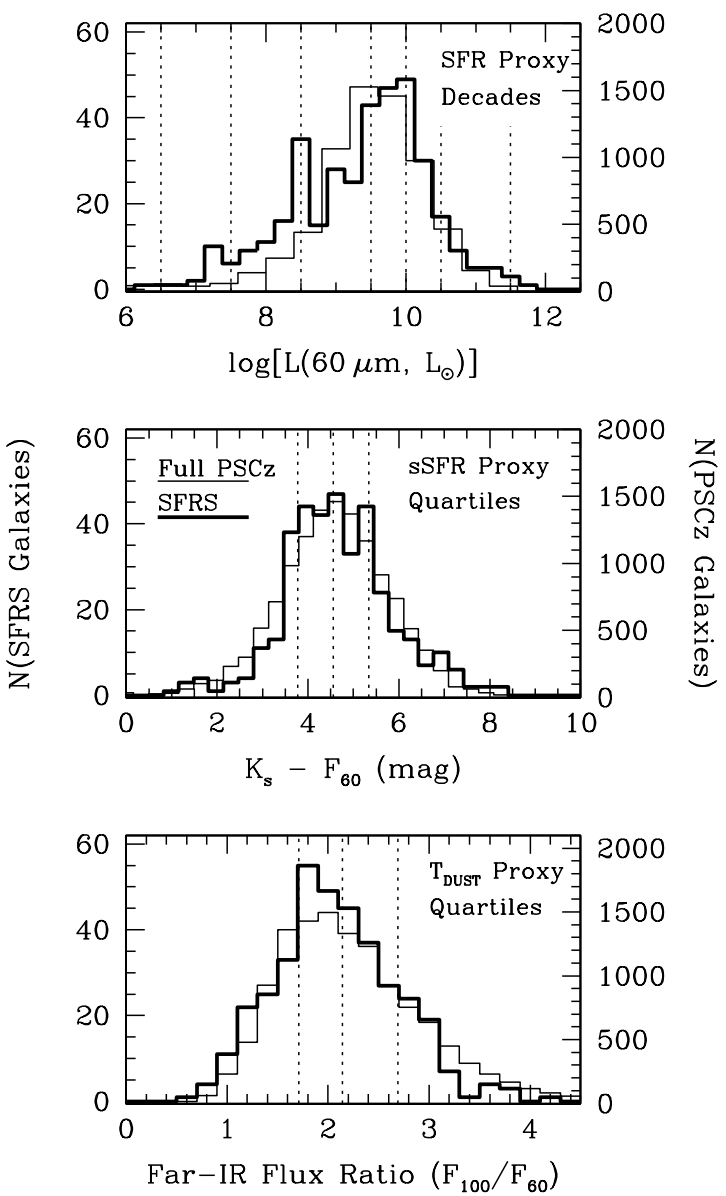

FIG. 2.- The distributions of the SFRS galaxies in each of the three parameter spaces used to select the sample. Thick histograms indicate the SFRS galaxies and are referenced to the left-hand axes, while thin histograms represent the larger PSCz sample from which the SFRS sample is drawn, and are referenced to the right-hand axes. The distributions are very similar in all three panels. The vertical dotted lines in each panel indicate the boundaries defining the bins (Tab. 1)

$60 \mu \mathrm{m}$ luminosity and by quartiles in both the $K_{s}-60 \mu \mathrm{m}$ color and the $F_{100} / F_{60}$ flux density ratio (Fig. 2). The bins are defined in Table 1. This ensured that the sample contains all existing combinations of high- and lowsSFR with far-IR color and luminosity. The changeover from cool dust/low sSFR to warm dust/high sSFR occurs around $L(60)=10^{9.5}$ to $10^{10.5} \mathrm{~L}_{\odot}$, which is the most heavily populated decade in luminosity. We therefore split this range into two half-decades in $L(60)$ to sample this important regime more finely. The result is $4 \times 4$ bins in each of two colors and eight bins in luminosity: 128 bins altogether.

After the bin boundaries were determined from the complete PSCz, a representative subsample was selected as follows. First, only galaxies with measured positive redshifts were included. Second, galaxies outside the Sloan Digital Sky Survey (SDSS) and NRAO VLA Sky Survey (NVSS; Condon et al. 1998) of the northern Galactic cap were excluded. This constraint ensures that visible and radio detections, and hence radioderived SFRs, would be available for all galaxies and simultaneously minimizes foreground confusion because the Galactic plane is $>20^{\circ}$ distant from all sources. Out 


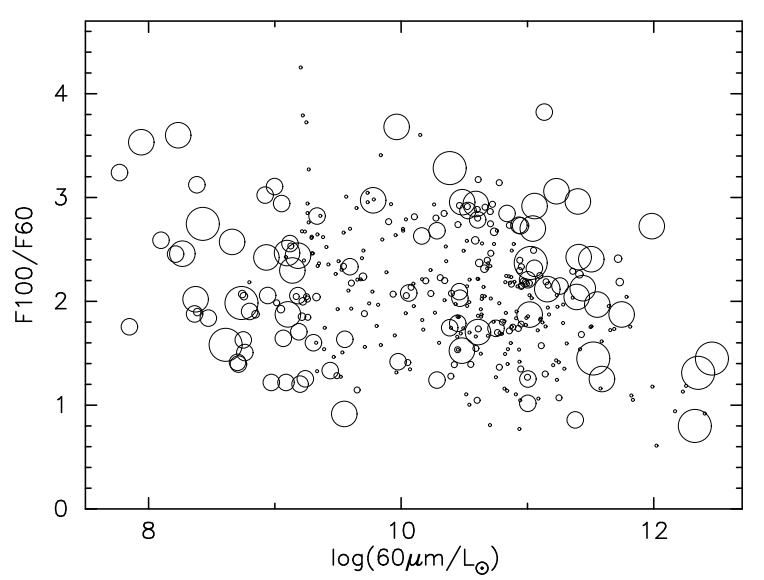

FIG. 3.- The three-dimensional SFRS galaxy distribution projected into the two-dimensional space defined by $60 \mu \mathrm{m}$ luminosity and far-IR flux density ratio. The symbol size is inversely proportional to weight: large symbols indicate relatively rare objects that occupy sparsely-populated bins; they significantly enlarge the parameter space explored by our sample and may be under-represented by programs not implementing selections similar to the SFRS.

of the 15,000 $\mathrm{PSC} z$ galaxies, 2564 meet these restrictions. Third, the few nearest galaxies were eliminated in order to avoid time-consuming Spitzer mapping of objects with large angular diameters and the attendant uncertainties in calculating global measurements. The exact distance limit was made luminosity-dependent: at $L(60)<10^{7.5} \mathrm{~L}_{\odot}$ and $L(60)>10^{10} \mathrm{~L}_{\odot}$, all galaxies were eligible because they were either sufficiently small or distant that they could be imaged efficiently with Spitzer. At intermediate luminosities, galaxies with recession velocities $c z<0.05 * L(60)^{1 / 2} \mathrm{~km} \mathrm{~s}^{-1}$ were excluded, where $L(60)$ is expressed in units of $\mathrm{L}_{\odot}$. This criterion excluded 194 galaxies. Because all these restrictions are based strictly on galaxy positions in three-dimensional space, they in no way bias the sample, and they ensure that low-luminosity sources are retained.

The final SFRS consists of a representative number of galaxies from each bin. The number chosen from each bin containing $N$ galaxies was $\sqrt{N}$, rounded up to a maximum of 10 galaxies. The specific galaxies chosen were the brightest within each bin. This selection sets the sample size at 369 galaxies and guarantees that the sample will be representative of the much larger $\mathrm{PSCz}$, and by inference, representative of star forming galaxies generally. The distributions of the sample galaxies in the two-dimensional projections of the three-dimensional selection space are illustrated in Figs. 3 and 4. The SFRS distributions are very similar in all three selection parameters (Fig. 2) to those for the full PSCz. The resulting sample is thus statistically well-defined, and covers the entire range of star formation properties seen locally: five decades in luminosity (and thus SFR); a factor of nearly 200 in specific SFR, and all masses, morphologies, and sizes.

The relative prevalence of a galaxy of any type within the sample is reflected in its weight: each SFRS galaxy in a given selection bin is assigned a weight determined by the ratio of the total number of $\mathrm{PSCz}$ galaxies in that bin to the number of sample galaxies drawn from that bin into the SFRS sample. Relatively rare galaxy types have low weights, while those from heavily populated

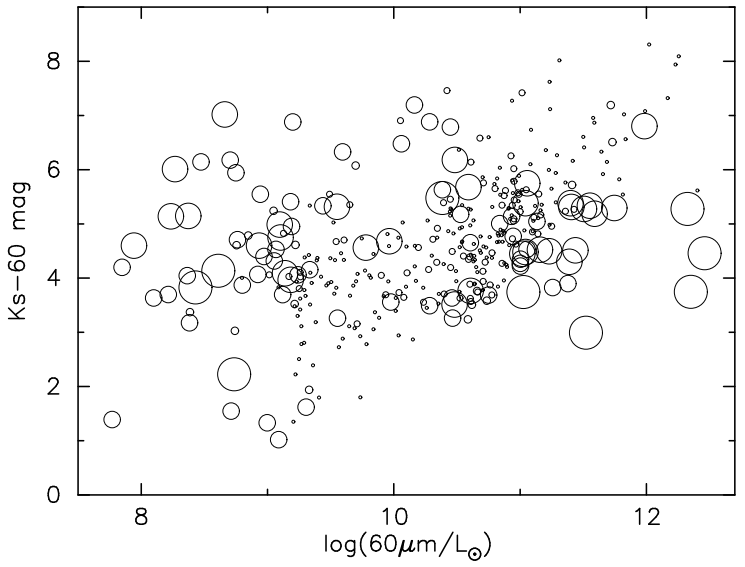

FIG. 4.- The three-dimensional SFRS galaxy distribution projected into the two-dimensional space defined by $60 \mu \mathrm{m}$ luminosity and near- to far-IR color (specific SFR proxy). Symbols as in Fig. 3.

bins have large weights. The names, positions, individual weights, and IRAS 60 and $100 \mu \mathrm{m}$ fluxes used to define the sample are given in Table 2 . These weights project the SFRS sample back to the parent $\mathrm{PSCz}$ population. Additional weighting based on the volume in which a galaxy would enter the PSCz would be needed to define a true volume-limited sample.

Because of the rigid selection criteria, the well-known quasar 3C 273 and the the blazar OJ 287 are members of the SFRS. Each is the only galaxy in its bin (weight三1). While we retain these objects to preserve the demographics of our selection, they can be ignored for studies relating purely to star formation.

\section{BASIC DATA}

In addition to the 2MASS and IRAS data used to define the selection criteria, many other resources are available. These are summarized in Table 3 and described in detail beginning with Sec. 3.2.

\subsection{Distances}

A significant fraction of the SFRS galaxies are very nearby, and can have peculiar velocities comparable to their Hubble flow velocities. Tully et al. (2008) have recently accumulated redshift-independent distance measurements for nearby galaxies by using alternate methods including the Tully-Fisher relation (Tully \& Fisher 1977), Cepheids (Freedman et al. 2001), the luminosity of stars at the tip of the red giant branch (Karachentsev et al. 2004, 2006), and surface brightness fluctuations (Tonry et al. 2001). These measurements yield quality distances for nearby $\left(v<3000 \mathrm{~km} \mathrm{~s}^{-1}\right)$ galaxies with distance modulus uncertainties $<0.1$ mag. Tully et al. (2008) estimated the distances of additional galaxies based on their association with groups/clusters. In total, their catalog ${ }^{13}$ includes 3,529 distance measurements (Tully, 2010, private communication.) for galaxies with $v<10,000 \mathrm{~km}$ $\mathrm{s}^{-1}$, of which 127 are in the SFRS.

For the 242 galaxies lacking quality distances, the heliocentric velocities in the PSCz catalog were converted to a corrected recession velocity taking into account the

\footnotetext{
13 The Extra-galactic Distance Database,
} 
TABLE 3

Summary of Avallable Data for SFRS Galaxies

\begin{tabular}{ccc}
\hline \hline Bandpass & Observatory & Sample Coverage \\
\hline $1.4 \mathrm{GHz}$ & VLA/NVSS & $100 \%$ \\
$12,25,60,100 \mu \mathrm{m}$ & IRAS & $100 \%$ \\
$65,90,140,160 \mu \mathrm{m}$ & AKARI FIR All-Sky Survey & $95 \%$ \\
$24 \mu \mathrm{m}$ & Spitzer/MIPS & $70 \%$ \\
$3.6,4.5,5.8,8.0 \mu \mathrm{m}$ & Spitzer/IRAC & $100 \%$ \\
$J H K_{s}$ & 2MASS & $100 \%$ \\
$u g r i z$ & SDSS & $100 \%$ \\
Optical Spectra & SDSS (fiber) & $57 \%$ \\
H $\alpha$ & NAOC & $30 \%$ (campaign ongoing) \\
$0.13-0.28 \mu \mathrm{m}$ & GALEX & $90 \%$ to date \\
& & \\
\hline
\end{tabular}

$\mathrm{Z}$

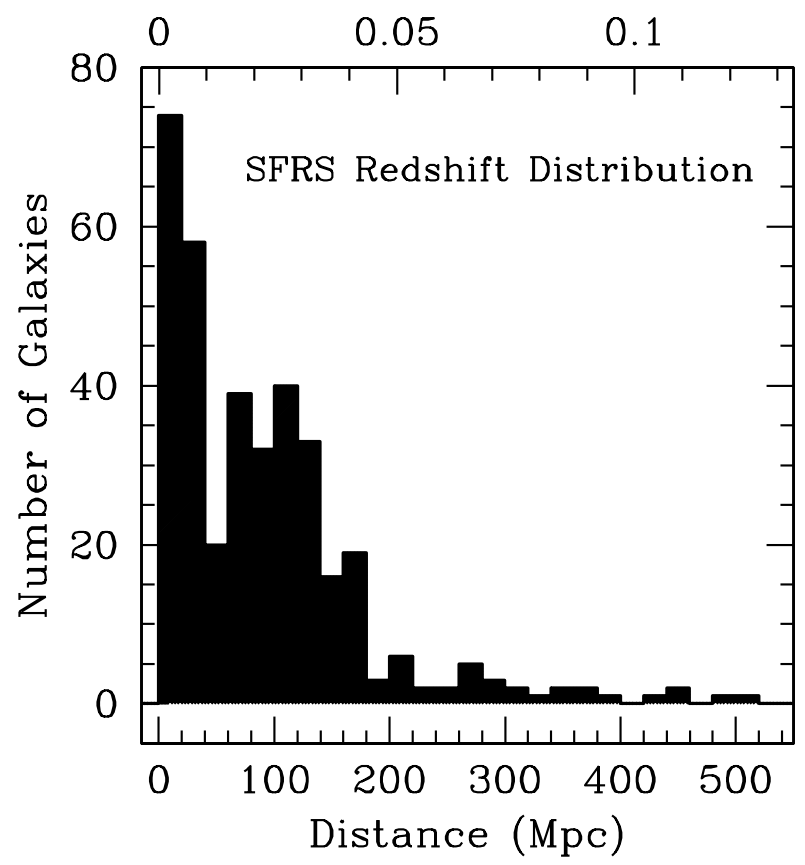

FIG. 5. - The distance/redshift distribution of the SFRS, in $20 \mathrm{Mpc}$ bins. Because of the $60 \mu \mathrm{m}$ IRAS selection, most of the sample galaxies are nearby $(z<0.05)$, but the distribution has a tail of higher-luminosity galaxies towards higher redshifts. The plot has been scaled to emphasize the nearby galaxies; the four most distant objects are not shown (3C 273, IRAS $11267+1558$, IRAS $13218+0552$, and OJ 287, at 660, 740, 850, and $1260 \mathrm{Mpc}$, respectively; Table 2).

velocity field of Virgo, the Great attractor and the Shapley supercluster, following Mould et al. (2000). All cataloged $\mathrm{PSC}$ heliocentric velocities agree with those provided by the NASA Extra-galactic Database (NED) ${ }^{14}$, within the uncertainties.

The resulting corrected velocities were used to estimate distances for all SFRS galaxies, assuming $H_{0}=73 \mathrm{~km}$ $\mathrm{s}^{-1} \mathrm{Mpc}^{-1}$. The distances are given in Table 2, and the overall distance distribution is plotted in Figure 5. The SFRS galaxies tend to be bright by virtue of their proximity $(60 \%$ are nearer than $100 \mathrm{Mpc}$, and $90 \%$ are closer than $180 \mathrm{Mpc}$ ) and are therefore easily accessible from a wide range of telescopes.

${ }^{14}$ http://ned.ipac.caltech.edu/

\subsection{IRAC Photometry}

The IRAC (Fazio et al. 2004) observations of most SFRS sources (i.e., all those lacking suitable archival data) were carried out during Cycle 5 (PID 50128) using standard observing parameters. Each of the 273 galaxies targeted by PID 50128 was observed with at least $6 \times 12 \mathrm{~s}$ full-array exposures using a cycling dither with a medium dither scale. All sources were observed in both IRAC fields of view, yielding a total exposure time of at least $72 \mathrm{~s}$ in each of the four IRAC bands at 3.6, 4.5, 5.8, and $8.0 \mu \mathrm{m}$. This relatively low exposure time consistently yielded $S / N>1000$ for these very bright galaxies. All sources not included in PID 50128 had already been observed by other programs, and the corresponding data were downloaded from the archive.

The archival and PID 50128 data were reduced together in as homogeneous a manner as possible. The basic IRAC data reduction was carried out by team members at the University of Western Ontario and at the Center for Astrophysics. In both instances it was based on the corrected Basic Calibrated Data (cBCD). The cBCD frames were object-masked and median-stacked on a per-AOR (Astronomical Observing Request) basis. The resulting stacked images were visually inspected and subtracted from individual cBCDs within each AOR. This was done to eliminate long-term residual images arising from prior observations of bright sources by the 3.6, 5.8, and $8.0 \mu \mathrm{m}$ arrays. The $4.5 \mu \mathrm{m}$ detector array did not suffer from residual images during Spitzer's cryogenic mission. Subtracting the median stacks also minimized gradients in the celestial backgrounds around each source. After these preliminaries, the data for each galaxy were mosaiced into four spatially-registered mosaics using IRACproc (Schuster et al. 2006). IRACproc augments the capabilities of the standard IRAC reduction software (MOPEX). The software was configured to automatically flag and reject cosmic ray hits based on pipeline-generated masks together with a sigma-clipping algorithm for spatially coincident pixels. IRACproc calculates the spatial derivative of each image and adjusts the clipping algorithm accordingly. Thus, pixels where the derivative is low (in the field) are clipped more aggressively than are pixels where the spatial derivative is high (point sources). This avoids downward biasing of point source fluxes in the output mosaics. The mosaics were resampled to 0 .'84 per pixel, so each pixel in the final mosaic subtends half the solid angle of the native IRAC pixels. 
Photometry was carried out with SExtractor (ver. 2.5.0; Bertin \& Arnouts 1996). Because our interest lies in global photometry and in particular in ensuring accurate global SEDs, we first registered all IRAC and GALEX mosaics (for which GALEX data were available; Sec. 3.7) to a common spatial scale using SWarp (version 2.17.1) and then applied SExtractor in two-image mode to photometer the galaxies. In two-image mode, SExtractor performs detection and characterization of the surface brightness distribution in one image and applies that surface brightness distribution to a second image. This was necessary because the infrared and ultraviolet mosaics in many instances have significant morphological differences; two-image mode prevents this fact from biasing the global color measurements. We used the IRAC $3.6 \mu \mathrm{m}$ mosaic - the best tracer of the stellar light distribution - as the detection image and photometered the GALEX and IRAC mosaics using the surface brightness distribution at $3.6 \mu \mathrm{m}$ to define the apertures. In a few cases where UV-bright features dominate, it was necessary to use the GALEX NUV image as the detection image in order to ensure that the SExtractor captures all the light in all six GALEX and IRAC bands. The IRAC photometry appears in Table 4.

\subsection{Radio Continuum Flux Densities}

Most of the radio observations come from the NVSS, a snapshot survey of $82 \%$ of the celestial sphere at $1.4 \mathrm{GHz}$ (Condon et al. 1998). The survey's key features are that it used VLA D-array, and the images were cleaned and restored with a $45^{\prime \prime}$ beam (Condon et al. 1998). Typical rms brightness fluctuations in the survey are $0.45 \mathrm{mJy} \mathrm{beam}^{-1}$, and the survey catalog reaches the $50 \%$ completeness level at $S=2.5 \mathrm{mJy}$. In practice, the SFRS sources were retrieved from the online catalog ${ }^{15}$ with a $30^{\prime \prime}$ search radius. Such queries returned results for 342 of the SFRS galaxies, all with unique matches. Figure 6 of Condon et al. (1998) shows a 1\% chance of finding a spurious match within this distance of an arbitrary position. Ten additional matches, two of them double, were found in a search radius of $60^{\prime \prime}$, and two additional matches were found with a $90^{\prime \prime}$ search. The probability of spurious matches at these distances is 5 $10 \%$, but such large position errors are highly unlikely for correct matches unless the source itself is very extended.

Condon et al. (2002) gave flux densities based on NVSS data for 192 of the SFRS galaxies. In all but 14 cases, the flux densities are essentially identical to the ones from the automated lookup. For the 14 discrepant cases and also for all cases where the NVSS position differed from the adopted galaxy position by more than $10^{\prime \prime}$, we examined the NVSS images ${ }^{16}$ together with the IRAS and IRAC infrared images. Most of the discrepancies were caused by blends of two radio sources, one of them being the SFRS galaxy and the second a nearby galaxy or QSO. This cause (blended sources) was found to explain discrepancies both in total measured flux and for discrepancies in the reported coordinates. In about half of the cases, the blended source was identifiable on the IRAC image or in NED. Most of the time, the IRAS (i.e.,

\footnotetext{
15 http://www.cv.nrao.edu/nvss/NVSSlist.shtml, lookup done in 2010 in catalog dated 2004

16 http://www.cv.nrao.edu/nvss/postage.shtml
}

SFRS) source was attributable to a single galaxy, but in some cases two galaxies are likely to contribute. The radio blends were deconvolved using IMFIT in the Common Astronomy Software Applications (CASA) package ${ }^{17}$, in each case with the minimal set of free parameters that led to a satisfactory fit ${ }^{18}$. Another cause of discrepancy was extended radio sources. The NVSS catalog fit Gaussians to all sources, but in some cases this is not an accurate description of the galaxy. In these cases, the radio flux was measured in a circular, rectangular, or polygonal beam as appropriate for the particular galaxy. In general, our results agree with those of Condon et al. (2002) but differ slightly because we took into account information from the IRAS images in determining how to fit the radio sources.

A final 16 SFRS sources either were not detected in the NVSS or had very low signal to noise. These were observed with the VLA in D configuration in 2008 July (program AA 319). ${ }^{19}$ The observations used the same observing frequencies and bandwidth as those of the NVSS but were $\geq 7$ minutes long as opposed to $30 \mathrm{~s}$ for the NVSS in the relevant declination bands. Data reduction was with CASA and followed the NVSS procedure in using superuniform weighting with $\mathrm{npix}=5$. The reductions differed in using 7".5 pixels and a restoring beam set by the actual baselines and weightings of each observation; typical beams were $30^{\prime \prime} \times 40^{\prime \prime}$. The two frequencies observed were imaged and cleaned separately and the resulting images averaged; the separate images were also inspected and source flux densities measured on each of them as well as on the combined image. This was especially important for three galaxies with bright radio sources (M87, 3C 273) in the outer part of the VLA primary beam. Flux densities were measured via Gaussian fitting (CASA IMFIT), by adding up pixels in rectangular areas, or where needed by deconvolution as discussed above. In many cases, the limiting noise source is sidelobes from strong, imperfectly cleaned sources in the field. The tabulated uncertainties are our best estimate taking these into account, especially by comparison of the images at the two frequencies and of different methods of measuring flux density.

All the radio flux densities are given in Table 5. Uncertainties are higher for sources marked as "extended" because the peak surface brightness is the best-measured parameter, and uncertainty in the source size contributes to the uncertainty in flux density. Unrecognized blends may give spuriously high flux densities, but this is likely to be the case for only a very few objects.

\subsection{MIPS 24 $\mu m$ Photometry}

Many SFRS galaxies are not well-detected by IRAS at 12 and $25 \mu \mathrm{m}$, leaving an obvious gap in the suite of useful SFR estimators (e.g., Calzetti et al. 2010). To fill this gap we photometered all available archival Spitzer/MIPS observations (Rieke et al. 2004) and were awarded time

\footnotetext{
17 http://casa.nrao.edu/index.shtml

18 When small-diameter objects could be identified, their positions were held fixed, and objects were forced to be point sources whenever possible. Extended objects had position angles held fixed when those could be determined from visible or IR data.

19 The AA 319 observations also included two galaxies that were in an early version of the SFRS sample but were later deleted.
} 
to observe the remainder of the sample via our own observing program (PID 50132, PI Fazio).

Our MIPS $24 \mu \mathrm{m}$ campaign was active from the start of 2008 November until the exhaustion of Spitzer's cryogen in 2009 May. A total of 178 SFRS galaxies were observed, all of them in Phot mode. Most used a small field size, $10 \mathrm{~s}$ exposures per position, and 2 cycles. For six relatively large galaxies, a large field size was used to ensure a sufficiently large source-free background was present in the final mosaics to permit an accurate background subtraction. In one instance (NGC 3338), a raster map had to be used to cover the full spatial extent of the source plus the nearby field.

All PID 50132 data were reduced using standard techniques. We used object-masked median stacks of all exposures of each target to eliminate array artifacts from the enhanced basic calibrated data (eBCD) before mosaicing. All mosaics were pixellated to 2.5 .

The archival observations were a heterogenous dataset employing a variety of exposure times and observing strategies. Our analysis of these 101 observations therefore began with the post-BCD data products, which retain some array-based artifacts but were nonetheless suitable for deriving global photometry. Two galaxies (NGC 4314 and 4418) exhibited saturation at their cores in the archival data, but apart from this we retrieved mosaics covering the full extent of a total of 101 of our sample galaxies. Together with the 178 objects from PID 50132, a total of roughly $3 / 4$ of the SFRS galaxies yielded useful mosaics.

We photometered all sources identically using SExtractor, accounting for differences in pixellation by an appropriate choice of convolution kernel. Typically the sources were detected with $S / N$ ratios of hundreds or even thousands in mosaics that were very far from being confused with unrelated background or foreground sources. Using the effective radii measured by SExtractor (KRON_RADIUS), we applied appropriate aperture corrections to the total fluxes following Table 4.13 of the MIPS Instrument Handbook. No special effort was made to identify and exclude foreground stars, but because of the typically very high galactic latitude of the sources this should not significantly bias the photometry.

The MIPS photometry was verified in two ways. First, all the SExtractor-generated background and object checkimages were inspected to make sure that the backgrounds were smooth on scales larger than the galaxies themselves and that SExtractor had identified all the pixels associated with a particular galaxy. Second, the results were compared to those reported for the 15 SFRS galaxies in common with the SINGS and LVLS samples (Table 6; Dale et al. 2007; Dale et al. 2009). All agree within $1 \sigma$ except for NGC 5474 and NGC 4395. The measurement for NGC 5474 (0.14 Jy) is $1.7 \sigma$ lower than the SINGS measurement (0.18 Jy). Among 15 independent measurements, a single discrepancy at the $1-2 \sigma$ level is acceptable agreement. NGC 4395 is arguably a different case because the photometry is discrepant at the $3 \sigma$ level. This galaxy is unusually extended with a particularly uneven surface brightness profile. The failure of the curve-of-growth to converge on a single level strongly suggested the MIPS photometry for NGC 4395 was biased low. For this single object the Dale et al. (2009) measurement was therefore adopted in preference to our own measurement.

The final aperture-corrected global MIPS $24 \mu \mathrm{m}$ photometry is given in Table 7 . The corresponding aperture corrections are given in Table 8 . Because the detections have such high $S / N$ ratios, the random (measurement) uncertainty is dominated by the systematic uncertainty in the absolute calibration of MIPS. According to the MIPS Instrument Handbook this uncertainty is between 4 and 8 percent; we therefore adopt $8 \%$ uncertainty as the most conservative estimate of the true error in all the MIPS photometry. For reasons having to do purely with scheduling, the galaxies lacking MIPS $24 \mu \mathrm{m}$ photometry tend to be those at lower Right Ascensions; eventually this lack will be corrected using photometry from the WISE mission.

\subsection{Far-Infrared Photometry from Planck}

Planck (Planck Collaboration 2011a) is a space-based mission now carrying out an all-sky survey in six bands from $25-1000 \mathrm{GHz}$ with a spatial resolution that progresses from $\sim 30^{\prime}$ to $5^{\prime}$ depending on the band. Although its main mission is to measure spatial anisotropies in the Cosmic Microwave Background, in the course of its repeated surveys of the sky it has detected thousands of foreground sources. The $1700+$ detections resulting from the first pass through the sky are tabulated in the Planck Early Release Compact Source Catalog (Planck Collaboration 2011b). Because the SFRS galaxies are bright, a significant number are detected by Planck: 176, 78, and 28 are detected by Planck's High-Frequency Instrument (Lamarre et al. 2010) at 350, 550, and $850 \mu \mathrm{m}$, respectively. This far-infrared photometry provides new and valuable constraints on the cold dust content of the detected SFRS galaxies, e.g., Planck Collaboration (2011c) found evidence for cold $(T<20 K)$ dust in their analysis of combined IRAS+Planck SEDs. The relevant photometry is presented in Table 7. No doubt the SFRS detection fraction will increase as Planck accumulates more complete passes over the sky and reaches correspondingly fainter flux limits.

\subsection{Visible and Near-Infrared Observations}

The visible photometry was taken from the data release 7 of the Sloan digital sky survey (SDSS; Abazajian et al. 2009). The SDSS consists of an imaging survey of $\pi$ steradians, mainly in the northern sky, in five passbands $u, g, r, i$, and $z$. The imaging was done in drift-scan mode, and the data were processed using the photometric pipeline Pното (Lupton et al. 2001) specially written for SDSS. All SFRS galaxies have photometry available in the five SDSS bands, although in six cases the cataloged values greatly understate the true values because they pertain only to the galaxy nuclei instead of the entire galaxy. We used the Petrosian magnitudes as the best measure of the total flux in the five SDSS bands (Blanton et al. 2001). The Petrosian magnitudes were calculated using the aperture set by the 'Petrosian radius' in the $r$-band, thus providing consistent measurements. SDSS Petrosian magnitudes should recover all the flux for an exponential galaxy profile independent of the axis ratio (Blanton et al. 2001) and about $80 \%$ of the flux for a de Vaucouleurs profile.

SDSS also acquired nuclear spectra for 210 SFRS galaxies. The spectra were obtained using two fiber- 
fed double spectrographs covering a wavelength range of 3800-9200 $\AA$. The resolution $\lambda / \Delta \lambda$ varies between 1850 and 2200. The SDSS fibers have a diameter of $3^{\prime \prime}$.

Near-infrared photometry was taken from the 2MASS extended source catalog. Although isophotal magnitudes had been used for the initial SFRS sample selection, total magnitudes were extracted from the 2MASS database for greater consistency with the global photometry measured in the other bands. 2MASS extrapolated total magnitudes are typically $\sim 0.3$ mag brighter than isophotal magnitudes. Typical measurement uncertainties are given as $0.03,0.04$, and $0.05 \mathrm{mag}$ at $J, H$, and $K_{s}$, respectively. Sec. 4.3.2 discusses the systematic biases in the 2MASS photometry.

The SDSS and 2MASS photometry is presented in Table 9 .

\subsection{GALEX Photometry in the Near-and Far-Ultraviolet Bands}

GALEX (Martin et al. 2005) is an astronomical satellite with sensitive wide-field ultraviolet imaging capability in two bands, the FUV (1350-1750^) and NUV (1750-2800A). The GALEX archive (release GR6) contains scientifically usable imaging (covering the full extent of our sources and therefore capable of yielding global flux measurements) in at least one of the GALEX bands for $332 / 369$ SFRS galaxies, or $90 \%$ of the sample. The fraction observed by GALEX in the NUV will increase as the survey portion of the mission continues, although FUV imaging is no longer possible.

Almost three-quarters of these imaging data in both wavebands were taken as part of GALEX's primary surveys $^{20}$ - the all sky survey (AIS) with an effective exposure time of $\sim 0.1 \mathrm{ks}$ and the relatively deep nearby galaxy survey (NGS) with an effective exposure time of $\sim 1.5 \mathrm{ks}$. With a resolution of $4^{\prime \prime}-6^{\prime \prime}$, GALEX images have lower spatial resolution than Spitzer/IRAC. However, since almost a third of the SFRS galaxies lie < $40 \mathrm{Mpc}$ away, together with a 1.25 degree GALEX field of view, the moderate GALEX spatial resolution nonetheless allows for robust measures of spatially-integrated fluxes and color which are comparable to data at other wavelengths. The GALEX observations and an analysis of the UV properties of the SFRS galaxies are described by S. Mahajan et al. (2012, in preparation; hereafter Paper 2).

\subsection{Ho Imaging from the National Astronomical Observatory of China}

In the spring of both 2008 and 2009, $\mathrm{H} \alpha$ imaging was acquired for SFRS sample galaxies from the NAOC $2.16 \mathrm{~m}$ telescope in Xinlong, Hebei Province, China. The Beijing Faint Object Spectrograph and Camera (BFOSC) was used to obtain on-source integrations lasting between 1800 and 3600s for each galaxy observed. The choice of exposure time was made based on prevailing conditions; longer exposures were used during brightsky time or for relatively faint targets. The $10^{\prime} \times 10^{\prime} .5$ BFOSC field of view illuminates a single CCD with $2048 \times 2080$ pixels. Each pixel subtends roughly 0'.3, well below the typical seeing during our campaign $\left(22^{\prime \prime} 0-22^{\prime \prime} 5\right)$. This spatial resolution is reasonably well-matched to the FWHM intrinsic to IRAC (1". $66-1$ 1".98). The BFOSC is equipped with a suite of 11 narrow $\mathrm{H} \alpha$ filters centered at wavelengths ranging from $6563 \AA$ to $7060 \AA$ at $50 \AA$ intervals. The filter bandpasses are $70 \AA$ wide. In the two observing campaigns carried out to date, a total of 105 SFRS galaxies were observed through one of these narrow-band $\mathrm{H} \alpha$ filters, the specific filter being chosen to cover the $\mathrm{H} \alpha+[\mathrm{NII}]$ complex at the target's redshift. Observations were also made of each galaxy through the standard Johnson $R$ band filter but with shorter exposure times $(400-600 \mathrm{~s})$ in order to measure the continuum flux.

The BFOSC data were reduced in the usual way. The lowest 32 rows of pixels were used for overscan correction. Standard IRAF tasks were used to subtract bias frames, construct and apply flatfields, coadd frames while simultaneously correcting for cosmic rays and known bad pixels using outlier rejection, and finally to photometer each of the 105 observed sources in both $\mathrm{H} \alpha$ and $R$. This work was carried out at the Chinese Academy of Sciences in Beijing. The quality of the final $\mathrm{H} \alpha$ mosaics is indicated by Fig. 6, which shows the outcome for UGC 8058, a typical case.

Figure 7 shows the relationship between the SFRs derived independently in $\mathrm{H} \alpha$ and the far-infrared for the 40 SFRS galaxies fully processed so far. The SFR estimates were made following the methods applied by Kewley et al. (2002) to 81 'pure' starburst galaxies in the Nearby Field Galaxy Survey (hereafter NFGS). Specifically, we estimated the $\mathrm{H} \alpha$-based SFR as $\operatorname{SFR}(\mathrm{H} \alpha)=7.9 \times 10^{-42} L(\mathrm{H} \alpha)$ and the far-infrared SFR as $\operatorname{SFR}(\mathrm{FIR})=4.5 \times 10^{-44} L($ TIR) (Kennicutt 1998), where we have estimated the total far-infrared luminosity $L(T I R)$ as described in Sec. 4.2.

Kewley et al. (2002) demonstrated that for 81 NFGS galaxies selected at visible wavelengths to cover a range of luminosities, $\operatorname{SFR}(\mathrm{H} \alpha)$ is discrepant with $\mathrm{SFR}(\mathrm{IR})$ in the sense that $\operatorname{SFR}(\mathrm{H} \alpha)$ tends to be significantly lower than SFR(IR). The discrepancy was measured to be a factor of nearly three $(2.7 \pm 0.3)$ and was shown to increase with increasing SFR. Both of those findings apply also to the SFRS galaxies shown in Fig. 7, but more so: as shown subsequently, the infrared-selected SFRS galaxies require a significantly larger mean reddening correction than did the NFGS galaxies to bring the $\mathrm{H} \alpha$ and farinfrared SFR indicators into agreement.

Most of the galaxies shown in Fig. 7 have GALEX photometry available. For all such objects an extinction correction $A_{H \alpha}$ appropriate for $\mathrm{H} \alpha$ was estimated based on the NUV photometry in Paper 2, which implemented the empirical IRX-based prescription following Eq. 1 of Buat et al. (2005). That estimate for $A_{N U V}$ was then converted into an $\mathrm{H} \alpha$ extinction estimate $A_{H \alpha}$ assuming a Calzetti (2001) dust law: $A_{H \alpha}=0.79\left(A_{V}+A_{M W}\right)=$ $0.79\left(0.77 \times A_{N U V}+A_{M W}\right)$ where $A_{M W}$ is the foreground extinction due to the Milky Way. Following this prescription the mean $\mathrm{A}_{H \alpha}$ was found to be $1.7 \mathrm{mag}$, corresponding to attenuation by a factor of 4.7. This likely reflects the fact that our sample is (primarily) infrared-selected, unlike the NFGS. A similar effect is seen for ultraviolet selection (Buat et al. 2005). Intriguingly, four galax- 

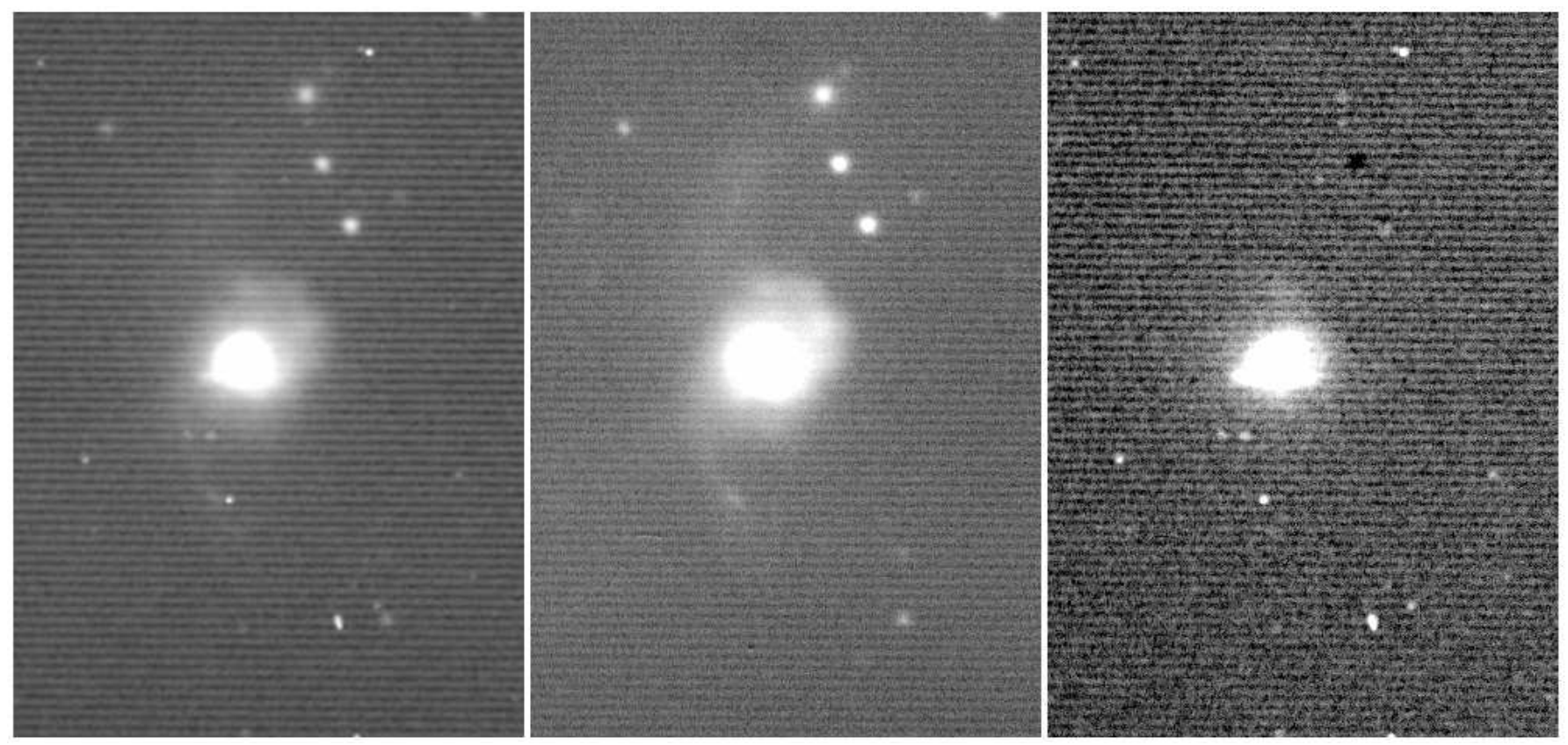

FIG. 6.- A typical example of the narrowband H $\alpha$ imaging being collected for the SFRS sample; the images shown here are for UGC 8058 . Left panel: the narrowband $\mathrm{H} \alpha$ emission-line image obtained at the NOAC. Center panel: the same field, seen in the continuum at $R$. Right panel: a difference image formed by subtracting the continuum image from the narrowband image, revealing the regions in which significant star formation is traced by the $\mathrm{H} \alpha$ emission.

ies (out of 34 with extinction estimates) lie more than an order of magnitude from the line of equality even when extinction-corrected. This suggests that for a minority of infrared-selected galaxies, either the intrinsic $\mathrm{H} \alpha$ emission does not accurately reflect the 'true' SFR. Alternately, the standard extinction corrections may not work well for such objects, as has been found for galaxies with large $\mathrm{SFR}(\mathrm{IR}) / \mathrm{SFR}(\mathrm{UV}$ ) ratios (Wuyts et al. 2011). The reason will remain unclear pending acquisition and analysis of $\mathrm{H} \alpha$ emission line intensities for the full SFRS. This will be possible as soon as the NAOC $\mathrm{H} \alpha$ imaging campaign is completed. In the summer of 2010 the BFOSC was upgraded with a new CCD having lower read noise. A subset of our team (led by $\mathrm{H}$. Wu) has been awarded time in the 2011 observing season to complete the imaging campaign for the roughly two-thirds of the SFRS for which $\mathrm{H} \alpha$ imaging has not yet been obtained.

We have also begun a longslit spectroscopic campaign (Sec. 4.3.1) to obtain dust reddening estimates for all SFRS galaxies via the Balmer decrement and thereby address the possibility that $A_{N U V}$ may not be the most reliable extinction indicator at visible wavelengths (Bell 2003). A detailed analysis of the global $\mathrm{H} \alpha$ emission line flux measurements for the full sample will be presented in a forthcoming paper (Y.-N. Zhu et al. 2012, in preparation).

\section{DISCUSSION}

\subsection{SFRS Compared to Other Nearby Galaxy Samples}

A number of recent survey programs have documented important aspects of star formation phenomenology. In addition to the studies mentioned above (SINGS, LVLS, and MESS), there are the NFGS (Kewley et al. 2002) and the Herschel Reference Survey (Boselli et al. 2010). The SFRS differs from these other efforts in significant ways.

SINGS is fundamentally different than SFRS because
TABLE 6

Galaxies in Common with Other SURVEYS

\begin{tabular}{lll}
\hline \hline \multicolumn{3}{c}{ Spitzer Infrared Nearby Galaxy Survey } \\
\multicolumn{3}{c}{ (SINGS) } \\
\hline NGC 3049 & NGC 3265 & NGC 5474 \\
NGC 3190 & NGC 3773 \\
\hline \multicolumn{3}{c}{ Local Volume Legacy Survey (LVLS) } \\
\hline NGC 2500 & NGC 4020 & NGC 5474 \\
NGC 2537 & NGC 4244 & NGC 5585 \\
NGC 2552 & NGC 4395 & \\
NGC 3274 & NGC 5204 & \\
& & \\
\multicolumn{3}{c}{ Herschel Reference Survey (HR) } \\
\hline NGC 3245 & NGC 4237 & NGC 4548 \\
NGC 3338 & NGC 4294 & NGC 4592 \\
NGC 3370 & NGC 4396 & NGC 4607 \\
NGC 3430 & NGC 4412 & NGC 4630 \\
NGC 3659 & NGC 4420 & NGC 4689 \\
NGC 3666 & NGC 4424 & NGC 4688 \\
NGC 3686 & NGC 4435 & NGC 4701 \\
NGC 3729 & NGC 4438 & NGC 4747 \\
NGC 4116 & NGC 4470 & UGC 8041 \\
NGC 4178 & NGC 4491 & NGC 5014 \\
NGC 4207 & NGC 4519 & NGC 5303 \\
& & \\
\hline
\end{tabular}

SINGS is comprised of a relatively small number (75) of very nearby, extended objects. SINGS therefore allows spatially-resolved measurements in numerous wavebands (Dale et al. 2007), but the SINGS galaxies sample primarily the low-luminosity end of the far-infrared luminosity function. This means SINGS isn't representative of star formation generally. Only five SINGS galaxies are present in the SFRS (Table 6).

The distinction between the LVLS and SFRS is less obvious because of the two-tiered optical+volume-limited 


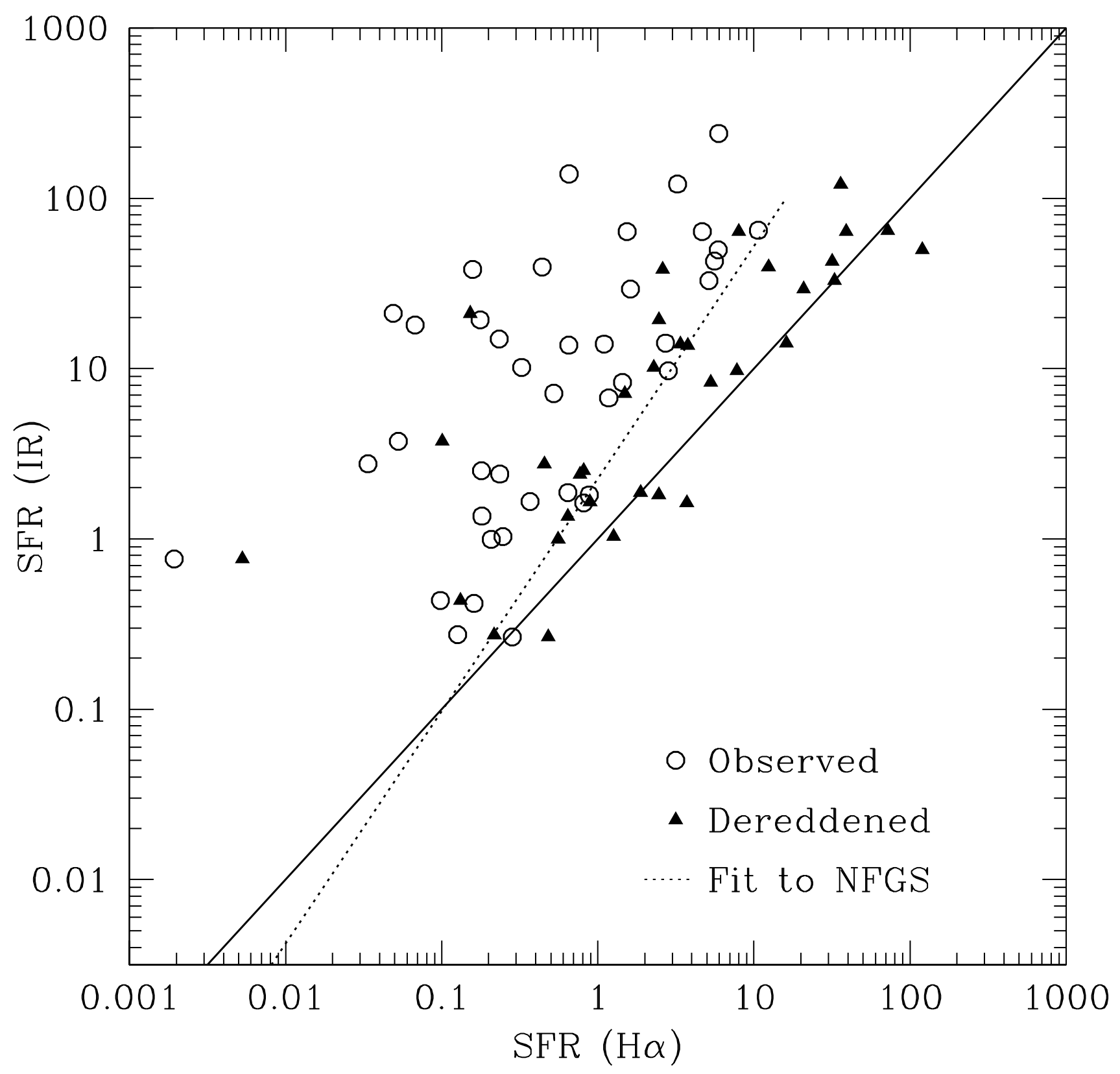

FIG. 7.- A comparison of far-infrared and $\mathrm{H} \alpha$ SFRs for an unrepresentative subset of the SFRS, following Fig. 1 of Kewley et al. (2002). Circles denote galaxies when SFR $(\mathrm{H} \alpha)$ is not correct for extinction due to dust. Solid triangles show the revised (larger) SFR(H $\alpha$ ) estimates that result when the extinction estimates from Paper 2 are applied. The solid line indicates where the points would fall if the Kennicutt (1998) relations were in agreement. The dashed line indicates the Kewley et al. (2002) fit to the corresponding data for the NFGS, not extinction-corrected.

nature of the selection used for the LVLS. The fundamental difference is that the LVLS is mostly comprised of lowluminosity dwarf galaxies, well below the break in the farinfrared luminosity function at $L_{F I R}=10^{10.25} \mathrm{~L} \odot$. Even so, the overlap between the LVLS and the SFRS samples is surprisingly small — only 10 objects (Table 6 ).

The MESS sample relies on a SDSS H $\alpha$ emission-line strength selection criterion to define a sample of 138 IRluminous galaxies that are very rapidly forming stars. MESS galaxy SFRs range from 11 to $61 \mathrm{M}_{\odot} \mathrm{yr}^{-1}$. Because it uses a single selection criterion, MESS does not control for ISM temperature or stellar mass and so can- not be representative of even high-intensity star formation generally. Furthermore, $\mathrm{H} \alpha$ fluxes systematically understate total SFRs compared to the far-infrared unless relatively large and uncertain extinction corrections are made, and MESS selects against star-forming galaxiess with large extinction. By design, MESS samples only galaxies with large SFRs.

GOALS (Armus et al. 2009) is more akin to the SFRS because it relies on a restricted $60 \mu \mathrm{m}$ brightness selection to construct the sample. All of the more than 200 GOALS galaxies are likewise bright, nearby objects for which an impressive range of multiband data have been 
made available, particularly HST and CXO imaging. But by design, GOALS selects for Luminous InfraRed Galaxies (LIRGs, galaxies with $L>10^{11} \mathrm{~L}_{\odot}$ ). The AGN fraction is significantly higher for high-luminosity galaxies. Indeed, it is a feature of the GOALS sample that it contains the full range of optical spectral types including many dominant AGNs as well as major-merger systems. While SFRS galaxies are by no means entirely free of AGN contributions (Sec. 4.2), they are a relatively minor contributor, and the fact that the SFRS spans the full range of both far-infrared color and luminosity simultaneously makes the SFRS less biased in characterizing star formation than GOALS.

The Herschel Reference Survey (Boselli et al. 2010) contains nearly as many galaxies as the SFRS (323 vs. 369) but does not capture the full range of star forming behavior because it is explicitly oriented toward highdensity environments. Consequently, it contains a much larger fraction of elliptical galaxies $(65 / 323)$ residing in Virgo and other major clusters. The IRAS $60 \mu \mathrm{m}$ detection fraction is about $3 / 4$, primarily because of a low detection fraction for HRS ellipticals. HRS contains 33 galaxies in common with the SFRS (Table 6), an overlap of about $10 \%$. Most important, HRS approximates two morphology-based volume-limited surveys, together spanning 'only' three decades in 60 micron luminosity. HRS has or soon will have a comprehensive suite of Herschel far-IR photometry available, but its selection makes it optimized for the study of how environment affects dust content rather than as an unbiased study of star formation.

\subsection{AGN Content}

The SFRS does not discriminate for AGN activity. For this reason it should be an excellent means of estimating AGN prevalence generally as well as the relative importance of AGNs for the overall energy budget of any farIR-selected sample, at least in the local Universe. Our ongoing campaign (Sec. 4.3.1) to acquire longslit spectra for all SFRS galaxies with the FAst Spectrograph for the Tillinghast Telescope (FAST; Fabricant et al. 1998) will eventually permit AGN detection to very faint levels via resolved emission line intensity ratios (e.g., Kewley et al. 2006). But even though the faint AGNs will be out of reach or a time, it is nonetheless possible to identify the relatively luminous AGNs immediately via their global infrared colors, and to exploit the spectroscopy that does exist to arrive at a preliminary estimate of AGN prevalence.

The most sensitive method for identifying AGNs is probably the BPT diagram (Baldwin, Phillips, \& Terlevich 1981; Kewley et al. 2001), an emission-line intensity ratio diagnostic that identifies AGNs via species that trace high-excitation conditions. Because it relies on visible lines, the BPT method will not find highlyobscured AGNs, but it can identify so-called 'transition' sources in which the contributions of star-forming activity and a central AGN are comparable. Figure 8 shows this method applied to a subsample of SFRS galaxies, i.e., all 165 objects for which the set of four necessary emission line intensities have been measured by SDSS. Of these 165 sources, 30 are identified as AGNs according to the Kewley et al. line-ratio criterion. Another 45 objects were observed spectroscopically by SDSS but do

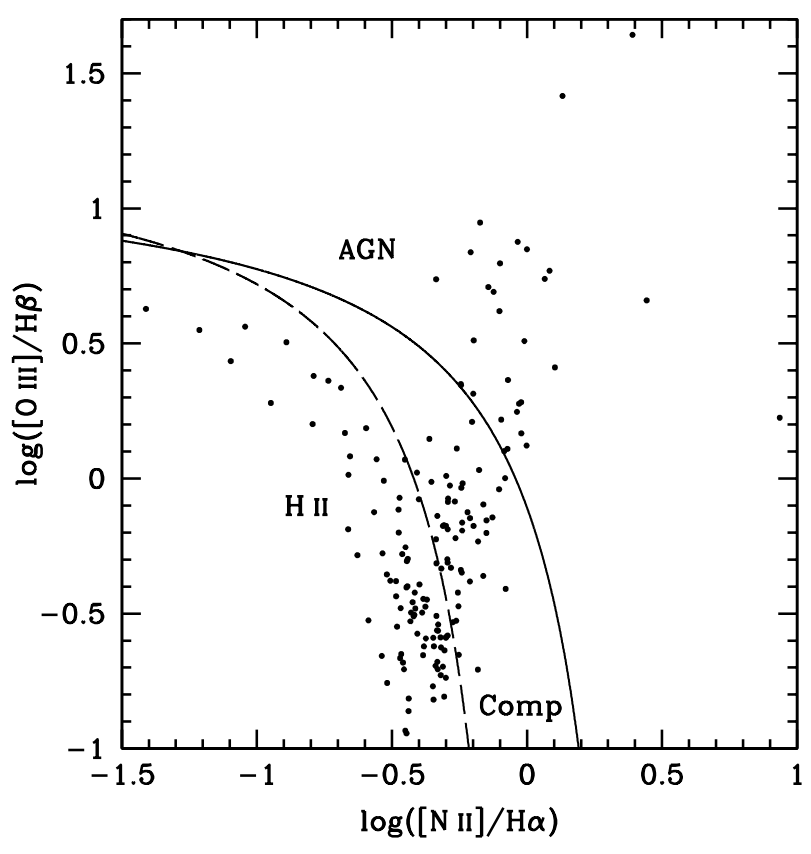

FIG. 8.- Line ratios from SDSS fiber spectra for 165 SFRS galaxies. Galaxies above the solid line are considered AGNs (Kewley et al. 2001), and those below the dashed line are considered purely star forming (Kauffmann et al. 2003), with emission-line intensity ratios closely matching those seen in HII regions. Those between the two lines are composite or transitional.

not exhibit the emission lines linked with AGNs. These 45 galaxies are unlikely to host strong AGNs. Thus the AGN fraction identified by the BPT diagram is roughly $1 / 7$.

Figure 9 shows the IRAC color-color plot first used by Stern et al. (2005) to empirically discriminate galaxies with infrared SEDs dominated by active nuclei from those dominated by star formation. It identifies 19 of the 369 SFRS galaxies ( $\sim 5 \%)$ as AGNs according to the empirical Stern et al. criteria. For these sources, emission from an active nucleus is a significant or even dominant contributor to the mid-IR SED. Moreover, some of the 18 galaxies outside the Stern et al. "wedge" but having redder [3.6] - [4.5] colors than the main group probably also contain AGNs. The most extreme such outliers are UGC 9560 (=II Zw 70) and UGC 5613, which have very different luminosities but are both strongly star-forming (Oconnell, Thuan, \& Goldstein 1978; Poggianti \& Wu 2000). An outlier with much smaller [5.8]- [8.0] color is IC 486 (=UGC 4155), which is a Sey 1 (Bonatto \& Pastoriza 1997). The outliers are thus a mixed group.

A third method to identify AGNs is based on the $25 \mu \mathrm{m}$ (or $24 \mu \mathrm{m}$ ) flux density (Sanders et al. 1988), specifically $F_{25} / F_{60}>0.2$. Despite their large $60 \mu \mathrm{m}$ fluxes, many SFRS galaxies are not reliably detected by IRAS in the $25 \mu \mathrm{m}$ band. We therefore used MIPS $24 \mu \mathrm{m}$ flux measurements, where available, to form the flux density ratio $F_{24} / F_{60}$, because MIPS $24 \mu \mathrm{m}$ fluxes track IRAS $25 \mu \mathrm{m}$ fluxes very closely on average (Dale et al. 2009). The distribution of the 313 SFRS galaxies with such data is shown in Figure 10. The distribution is qualitatively similar to that of the Revised Bright Galaxy Survey (Sanders et al. 2003). Only a small minority of the sources $(22 / 313$, or about $7 \%)$ lie at $F_{24} / F_{60}>0.2$, 


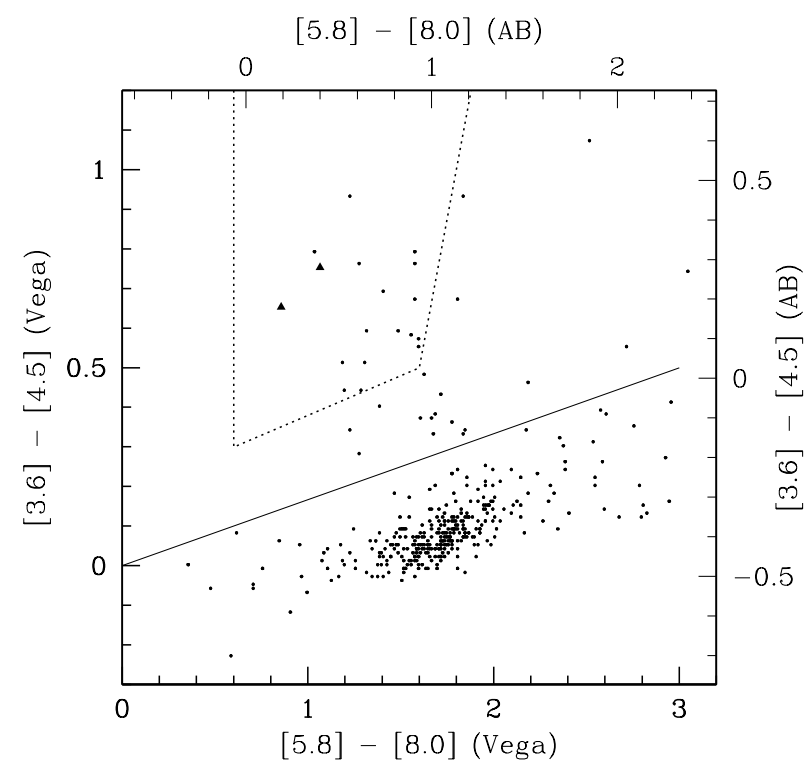

FIG. 9.- IRAC colors of SFRS galaxies. Colors are shown in both Vega and AB magnitudes on opposite axes. The solid triangles denote 3C 273 and OJ 287. The dotted line encompasses the empirically-determined region within which galaxy colors indicate the presence of an AGN (Stern et al. 2005). The solid line indicates the division between normal galaxies and outliers, which probably represent a mix of AGNs and strongly star-forming systems.

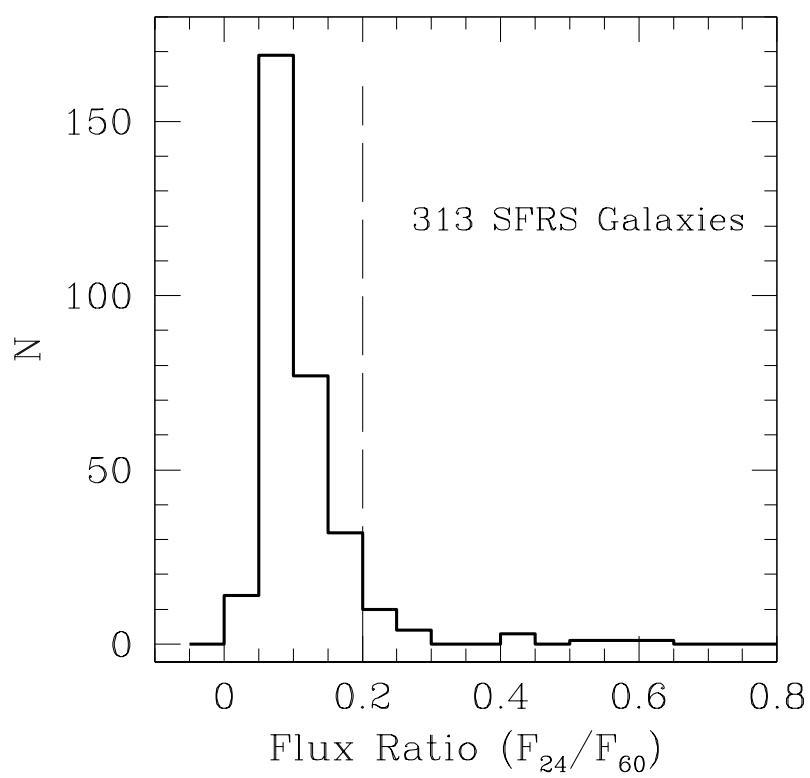

FIG. 10.- The distribution of the ratio $F_{24} / F_{60}$ for 313 SFRS galaxies for which MIPS $24 \mu \mathrm{m}$ fluxes (or their IRAS $25 \mu \mathrm{m}$ equivalents) are available. The vertical line marks the value above which Sanders et al. (1988) suggest that a relatively large mid-infrared flux indicates that an AGN contributes to the SED. Out of 313 SFRS galaxies 22 meet this criterion. They are listed in Table 10. which Sanders et al. (1988) identify as potentially arising from a significant contribution coming from an active nucleus. Most of the galaxies identified as AGNs in this way are also identified by one of the other two methods (Table 10).

No matter how they are identified, the AGNs in the SFRS do not have a noticeably different $F_{60} / F_{100}$ ratio than the non-AGNs. Figure 11 is a far-infrared colorcolor diagram showing the 313 SFRS galaxies with either IRAS $25 \mu \mathrm{m}$ or MIPS $24 \mu \mathrm{m}$ flux measurements, including the 22 objects identified as AGNs by the Sanders et al. (1988) criterion. This suggests that for most galaxies in the sample, AGNs contribute relatively little of the far-infrared light. Conversely, the far-infrared emission is an uncontaminated tracer of star formation. This result is consistent with Mullaney et al. (2011), who found that the SEDs of AGNs drop rapidly at wavelengths longer than $40 \mu \mathrm{m}$. It is also consistent with the outcome of Netzer et al. (2007), who found that the far-infrared properties of QSOs is almost entirely due to star formation. This view is confirmed by an examination of the FIR-radio correlation (e.g., Condon et al. 1992, Helou et al. 1985), the fact that the non-thermal radio emission and the thermal far-infrared emission correlate tightly over at least four orders of magnitude in far-infrared luminosity. Fig. 12 shows the FIR-radio correlation constructed using total infrared flux estimates $F(T I R)=$ $2.403 \nu f_{\nu}(25 \mu \mathrm{m})-0.2454 \nu f_{\nu}(60 \mu \mathrm{m})+1.347 \nu f_{\nu}(100 \mu \mathrm{m})$ in the usual way (Dale \& Helou 2002) except that we employ MIPS $24 \mu \mathrm{m}$ fluxes where available instead of the less precise IRAS $25 \mu \mathrm{m}$ fluxes. The solid line in the lower panel of Fig. 12 is an unweighted least-squares fit to all SFRS galaxies (except 3C 273 and OJ 287, which have extremely high radio continuum luminosities arising from dominant central AGNs). If all galaxies flagged as hosting AGNs by at least one of the three criteria above are excluded from the fit, the slope changes very little (to 1.09 from 1.086) and the scatter about the fit does likewise (decreasing to 0.29 dex from $0.31 \mathrm{dex}$ ). Thus, if the two dominant AGNs are excluded from the fit, the FIR-radio correlation is not significantly affected by the presence of AGNs in 52/367 (roughly one of every seven galaxies). This underlines the utility of the thermal farIR as an optimal probe of SFR.

The FIR-radio correlation obtained for the SFRS sample is higher than unity but nonetheless shallower than reported by Devereux \& Eales (1989), roughly 1.1 instead of 1.28. This could be due to selection effects: Devereux \& Eales (1989) employed an optical selection that sampled luminosities only up to $\sim 10^{11} \mathrm{~L}_{\odot}$. Yun, Reddy, \& Condon (2001) find a slope much closer to unity for their sample of bright infrared-selected galaxies, a result later confirmed by Bell (2003).

In summary, the three different AGN detection criteria identify three different AGN subsamples comprising from 5 to $10 \%$ of the SFRS study sample. There is only modest overlap among the three criteria (Table 10), which in hindsight validates the use of multiple tracers of AGN activity. AGNs are more prevalent at higher luminosities, despite the fact that they contribute little to the total far-infrared luminosities of star-forming galaxies. AGNs were detected in $\sim 15 \%$ of the sample (52/369 galaxies) when all three detection methods were combined. Taken 
TABLE 10

AGN Detections

\begin{tabular}{|c|c|c|c|c|}
\hline \multirow[t]{2}{*}{ Number } & \multirow[t]{2}{*}{ Name } & \multicolumn{3}{|c|}{ Detection Method } \\
\hline & & Emission Lines & $F_{24} / F_{60}$ & $3.6-8.0 \mu \mathrm{m}$ Colors \\
\hline 1 & IC 486 & $\mathrm{Y}$ & $\mathrm{Y}$ & $\ldots$ \\
\hline 7 & IRAS $08072+1847$ & $\ldots$ & Y & $\ldots$ \\
\hline 30 & OJ 287 & $\mathrm{Y}$ & $\mathrm{Y}$ & $\mathrm{Y}$ \\
\hline 32 & IRAS $08550+3908$ & $\mathrm{Y}$ & $\ldots$ & - \\
\hline 36 & IRAS $08572+3915 \mathrm{SW}$ & $\ldots$ & $\ldots$ & $\mathrm{Y}$ \\
\hline 55 & NGC 2893 & $\ldots$ & $\mathrm{Y}$ & - \\
\hline 57 & CGCG 238-066 & $\mathrm{Y}$ & $\mathrm{Y}$ & - \\
\hline 64 & CGCG 182-010 & $\ldots$ & $\mathrm{Y}$ & - \\
\hline 76 & IC 2551 & $\ldots$ & $\mathrm{Y}$ & - \\
\hline 79 & IRAS $10120+1653$ & $\mathrm{Y}$ & $\ldots$ & $\ldots$ \\
\hline 91 & UGC 5713 & $\ldots$ & Y & $\mathrm{Y}$ \\
\hline 100 & UGC 5881 & $\mathrm{Y}$ & $\ldots$ & $\ldots$ \\
\hline 107 & CGCG 95-055 & $\mathrm{Y}$ & $\ldots$ & $\ldots$ \\
\hline 109 & UGC 6074 & $\ldots$ & $\mathrm{Y}$ & ... \\
\hline 118 & IC 2637 & $\mathrm{Y}$ & $\ldots$ & $\ldots$ \\
\hline 121 & IRAS $11167+5351$ & $\ldots$ & $\ldots$ & $\mathrm{Y}$ \\
\hline 122 & NGC 3633 & $\ldots$ & $\mathrm{Y}$ & $\ldots$ \\
\hline 139 & NGC 3758 & $\ldots$ & $\ldots$ & $\mathrm{Y}$ \\
\hline 169 & UGC 7016 & $\mathrm{Y}$ & $\ldots$ & $\ldots$ \\
\hline 189 & NGC 4253 & $\cdots$ & $\mathrm{Y}$ & $\mathrm{Y}$ \\
\hline 194 & NGC 4385 & $\ldots$ & $\mathrm{Y}$ & . \\
\hline 198 & NGC 4418 & $\ldots$ & $\mathrm{Y}$ & $\mathrm{Y}$ \\
\hline 204 & $3 \mathrm{C} 273$ & $\ldots$ & $\mathrm{Y}$ & $\mathrm{Y}$ \\
\hline 233 & MCG 8-23-097 & $\mathrm{Y}$ & $\ldots$ & $\ldots$ \\
\hline 239 & UGC 8058 & $\ldots$ & $\mathrm{Y}$ & $\mathrm{Y}$ \\
\hline 244 & NGC 4922 & $\ldots$ & $\ldots$ & $\mathrm{Y}$ \\
\hline 248 & UGC 8269 & $\mathrm{Y}$ & $\ldots$ & $\ldots$ \\
\hline 253 & IRAS $13144+4508$ & $\mathrm{Y}$ & $\mathrm{Y}$ & $\ldots$ \\
\hline 259 & NGC 5104 & $\mathrm{Y}$ & $\ldots$ & $\ldots$ \\
\hline 263 & IRAS $13218+0552$ & $\ldots$ & $\ldots$ & $\mathrm{Y}$ \\
\hline 270 & IRAS $13349+2438$ & $\ldots$ & $\mathrm{Y}$ & $\ldots$ \\
\hline 276 & MK 268 & $\mathrm{Y}$ & $\ldots$ & $\mathrm{Y}$ \\
\hline 277 & NGC 5278 & $\mathrm{Y}$ & $\ldots$ & $\cdots$ \\
\hline 278 & NGC 5273 & $\mathrm{Y}$ & $\ldots$ & $\ldots$ \\
\hline 281 & UGC 8696 & $\mathrm{Y}$ & $\ldots$ & $\mathrm{Y}$ \\
\hline 283 & MK 796 & $\ldots$ & $\ldots$ & $\mathrm{Y}$ \\
\hline 284 & IRAS $13446+1121$ & $\mathrm{Y}$ & $\ldots$ & $\mathrm{Y}$ \\
\hline 286 & NGC 5313 & $\mathrm{Y}$ & $\ldots$ & $\ldots$ \\
\hline 288 & NGC 5347 & $\mathrm{Y}$ & $\mathrm{Y}$ & - \\
\hline 292 & UGC 8850 & $\ldots$ & $\mathrm{Y}$ & $\mathrm{Y}$ \\
\hline 296 & NGC 5403 & $\mathrm{Y}$ & $\ldots$ & $\ldots$ \\
\hline 303 & CGCG 074-129 & $\mathrm{Y}$ & $\mathrm{Y}$ & $\mathrm{Y}$ \\
\hline 309 & IC 4395 & $\mathrm{Y}$ & $\ldots$ & - \\
\hline 322 & UGC 9412 & $\ldots$ & $\mathrm{Y}$ & $\mathrm{Y}$ \\
\hline 329 & IRAS $14538+1730$ & $\mathrm{Y}$ & $\ldots$ & $\ldots$ \\
\hline 332 & UGC 9639 & $\mathrm{Y}$ & $\ldots$ & $\ldots$ \\
\hline 339 & IC 4553 & $\mathrm{Y}$ & $\ldots$ & - \\
\hline 341 & IC 4567 & $\mathrm{Y}$ & $\ldots$ & $\ldots$ \\
\hline 343 & NGC 5975 & $\mathrm{Y}$ & $\ldots$ & $\ldots$ \\
\hline 350 & UGC 10120 & $\ldots$ & $\mathrm{Y}$ & $\mathrm{Y}$ \\
\hline 352 & NGC 6040 & $\mathrm{Y}$ & $\ldots$ & $\ldots$ \\
\hline 358 & IRAS $16150+2233$ & $\mathrm{Y}$ & $\ldots$ & $\ldots$ \\
\hline & $\begin{array}{l}\text { Total } \\
\text { al }\end{array}$ & 30 & $\begin{array}{l}22 \\
52\end{array}$ & 19 \\
\hline
\end{tabular}

as a whole, these results suggest that the far-infrared selection described above (Sec 2) has successfully yielded a sample with bolometric luminosities dominated by star formation, and there is no compelling evidence that the AGNs significantly contaminate the far-infrared SEDS.

\subsection{Pending Observing Campaigns}

There are several ongoing observing campaigns for which significant data have been acquired but which are not yet complete. They are described below.

\subsubsection{Longslit Optical Spectroscopy with FAST}

Visible spectra from SDSS are available only for a fraction of the SFRS galaxies (Abazajian et al. 2009). More- over, the SDSS spectra were obtained through fibers centered on the nuclei and so do not uniformly measure or constrain the excitation conditions within the galaxy disks. We are therefore reobserving SDSS galaxies with a long-slit spectrograph in order to: 1.) build a consistent set of disk+nuclear spectra with better sky subtraction, 2.) take advantage of the spatial dimension to implement accurate starlight subtraction, and 3.) measure rotation curves.

The observations are currently being carried out at the Fred Lawrence Whipple Observatory $1.5 \mathrm{~m}$ telescope at Mt. Hopkins with FAST. FAST is being used with a $2^{\prime \prime}$ wide slit positioned along the major axis of the 


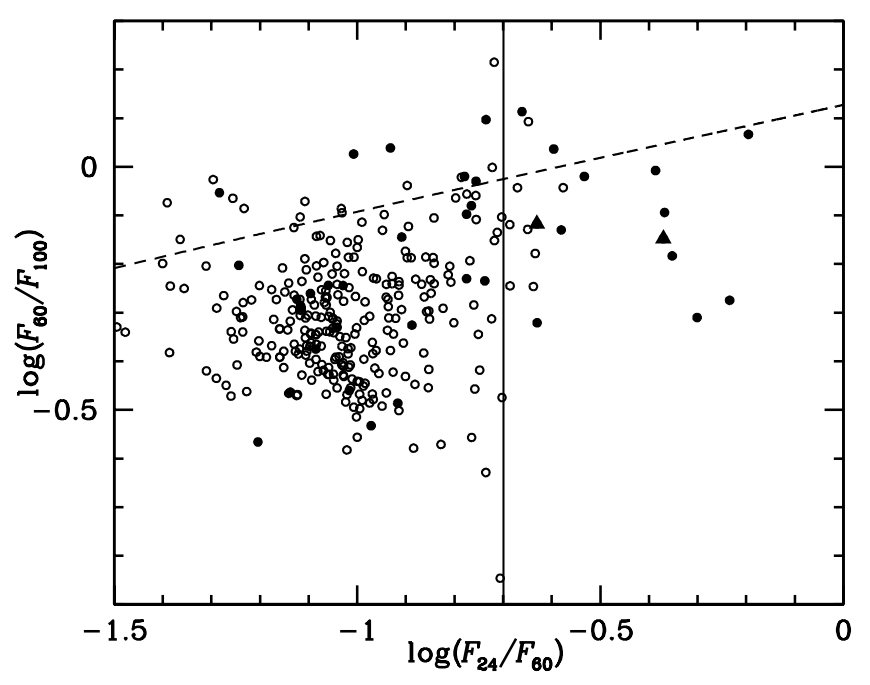

FIG. 11.- Far-infrared color-color diagram for 313 SFRS galaxies. The $24 \mu \mathrm{m}$ flux densities come from MIPS if available or IRAS $(25 \mu \mathrm{m})$ otherwise, and the 60 and $100 \mu \mathrm{m}$ flux densities come from IRAS. Galaxies to the right of the vertical line meet the Sanders et al. (1988) criterion for an active nucleus that affects the far-infrared colors. The dashed line indicates the colors of isothermal dust with emissivity $\epsilon \propto \nu^{-1}$. Filled symbols indicate galaxies identified as AGN on the basis of nuclear SDSS spectra (Fig. 8) or the Stern diagram (Fig. 9). The solid triangles correspond to 3C 273 and OJ 287. The 56 SFRS galaxies having only upper limits at $25 \mu \mathrm{m}$ are not plotted, but only 27 of them have $25 \mu \mathrm{m}$ upper limits large enough to potentially allow them into the AGN territory.

galaxies. For galaxies which extend beyond the slit ends we obtain either a second spectrum along the minor axis of the galaxy, or a second spectrum at a sky position away from the galaxy. The spectra are obtained with a $600 \mathrm{l} / \mathrm{mm}$ grating in two tilt positions in order to cover the blue $(3700-5700 \AA)$ and the red $(5500-$ $7500 \AA$ ) part of the spectrum with resolutions of $2.2 \AA$ and $2.7 \AA$ respectively. Throughout the modest redshift range occupied by our sample, this setup covers the standard diagnostic emission lines in the optical band: [O II] $\lambda 3727 \AA ; \mathrm{H} \beta$; [O III] $\lambda \lambda 4959,5007 \AA$; [O I] $\lambda 6300 \AA$; $[\mathrm{N}$ II] $] \lambda \lambda 6548,6583 \AA ; \mathrm{H} \alpha$; and $[\mathrm{S}$ II] $\lambda \lambda 6716,6731 \AA$. The exposure time for each nucleus has been chosen in order to achieve a signal-to-noise ratio $(S / N)$ of at least 40 at the $\mathrm{H} \alpha$ line, based on the available photometry from SDSS.

\subsubsection{PAIRITEL Near-IR Imaging}

As described in Sec. 2, 2MASS $K_{20}$ magnitudes were used as a stellar mass proxy to define the SFRS sample because of the well-characterized and uniform data quality and the full-sky coverage. However, a campaign has been initiated to replace the 2MASS photometry with significantly deeper near-IR observations in the same three bands, because the 2MASS observations are somewhat shallow. They are not optimal for detecting the faint outskirts of even nearby galaxies, where relatively high sky backgrounds obscure the outermost, low surface-brightness features. This leads to a systematic downward bias in the 2MASS photometry that is at present not fully characterized. Kirby et al. (2008) found the discrepancy to be highly variable, ranging up to $2.5 \mathrm{mag}$ in extreme cases. Karachentsev et al. (2002)

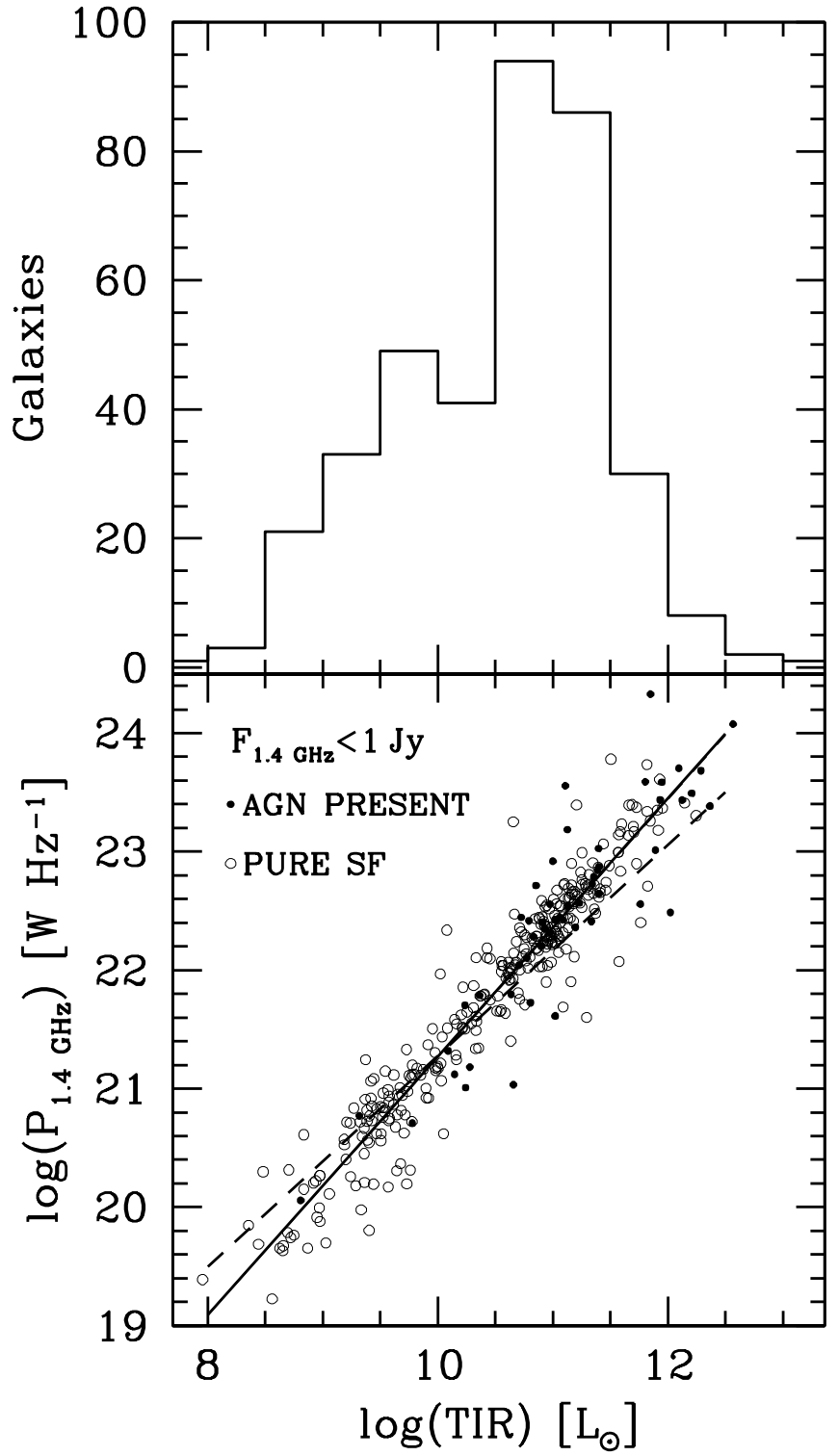

FIG. 12.- Upper panel: Histogram of total infrared luminosities calculated as described in the text. Lower panel: The FIR-radio correlation for 367 galaxies with radio continuum fluxes below $1 \mathrm{Jy}$, i.e., all but the two SFRS galaxies (3C 273 and OJ 287) dominated by emission from an AGN at radio wavelengths. Open circles indicate the 52 sources (Table 10) for which at least one of the three AGN criteria described in Sec. 4.2 is met. Solid circles indicate sources not known to have any AGN contribution. The line is an unweighted least-squares fit to the entire distribution. The same fitting procedure, when performed only for the 315 'pure' starforming galaxies, yields a correlation indistinguishable from that for all 367 galaxies on the scale of this plot. The dashed line illustrates a slope of unity.

found that the global 2MASS XSC photometry implied unphysical colors in some cases.

Deeper near-infrared observations will offer several advantages. They will greatly reduce both the measurement uncertainties and the bias in the existing photometry. They will better characterize the true extents and morphologies of the SFRS galaxies. They will also be a much better match to the existing Spitzer/IRAC data, which benefit from the very low backgrounds available 
from space. This will facilitate accurate K-corrections and stellar mass estimates planned for subsequent papers as well as reliable bulge/disk decompositions (because a greater extent and dynamic range in disk surface brightness is sampled).

Motivated by these considerations, the SFRS team sought and was awarded time for near-infrared imaging in 2009, 2010, and 2011 with the Peterson Automated Infrared Imaging Telescope (PAIRITEL; Bloom et al. 2006), the same telescope originally used for 2MASS. Useful $J H K_{s}$ imaging (i.e., flattened, backgroundsubtracted, and astrometrically correct mosaics encompassing all the emission from the targets) has been acquired for 259 SFRS galaxies to date. Exposure times were typically $20 \mathrm{~min}$ on-source, but in some cases the exposures were shorter because of circumstances related to weather or instrumentation. The PAIRITEL observations typically reach two magnitudes deeper than 2MASS. Based on a preliminary analysis of the $K_{s}$ imaging data reduced to date, it appears that the total magnitudes listed in Table 9 understate the galaxies' true output by about $0.3 \mathrm{mag}$ (P. Bonfini, 2011facility, private communication). The goal is to complete the deep $J H K_{s}$ coverage in the 2012a observing semester, after which the imaging and photometry will be presented together with a structural decomposition of the SFR galaxies (P. Bonfini et al., 2012, in preparation).

\subsubsection{Far-Infrared Detections by AKARI}

$A K A R I$ is a cryogenic space-based infrared telescope facility launched in 2006. Because it uses infrared detectors with pixels smaller $(0.5-0.9$; Jeong et al. 2007) than those used by IRAS, AKARI offers spatial resolution superior to that of IRAS despite having a primary mirror only slightly larger. For example, the Far-Infrared Surveyor (FIS) point spread functions have FWHMs ranging from $40^{\prime \prime}$ to $60^{\prime \prime}$ depending on the band (Kawada et al. 2007). AKARI therefore offers a way to improve upon IRAS photometry and likewise improve the fidelity of the far-IR SFRs inferred for our sample in cases of high foreground gradients arising from Galactic cirrus and/or confused IRAS sources, because AKARI has the potential to resolve out such contributions to the IRAS flux measurements.

Two all-sky surveys were carried out by the AKARI mission, covering $>94 \%$ of the sky more than twice $(\mathrm{Mu}-$ rakami et al. 2007). The central wavelengths of the six survey bands are 9 and $18 \mu \mathrm{m}$ with the Infrared Camera (IRC; Ishihara et al. 2010) and 65, 90, 140, and $160 \mu \mathrm{m}$ with the FIS (Kawada et al. 2007). All SFRS galaxies were detected in both the FIS and IRC all-sky surveys, which reached detection limits of 0.21 Jy at the $80 \%$ completeness level at $18 \mu \mathrm{m}$ and a $5 \sigma$ detection limit of $0.55 \mathrm{Jy}$ at $90 \mu \mathrm{m}$. The $90 \mu \mathrm{m}$ band is the most sensitive of the four FIS bands and covers a bandpass very similar to the IRAS $100 \mu \mathrm{m}$ band. A subsequent paper (H. Kaneda et al., in preparation) is planned to refine the far-IR SFR estimates with the full suite of $A K A R I$ photometry.

\section{CONCLUSION}

The Star Formation Reference Survey, by virtue of its reliance on a restricted but representative far-IR selection, ensures the capability to study obscured star formation in all of its varied manifestations in the local Universe. Its panchromatic resources, spanning UV to radio wavelengths and featuring $100 \%$ complete photometry in the visible to mid-infrared regimes (ugrizJHK $K_{s}$ and four IRAC bands) provides an opportunity to quantitatively assess the degree to which far-IR emission reflects total (not just obscured) SFR. This comprehensive collection of the most widely-used SFR indicators will furnish an invaluable resource for the interpretation of more distant galaxies, i.e., galaxies for which some or even nearly all such SFR metrics are inaccessible because of their relative faintness, so that a context exists in which to better understand the limited data available. Because the SFRS is fully representative of star-forming galaxies in the local Universe, it is an optimal benchmark for understanding star formation in the distant cosmos.

The interplay of various star formation and AGN indicators will be explored in future papers. Paper 2 will examine the relationship of UV SFR indicators to those obtained in the other bands. P. Bonfini et al. (2012, in preparation) will describe the methods whereby relatively faint AGNs in the sample are identified using structural decomposition. Y.-N. Zhu et al. (2012, in preparation) will examine a suite of narrow-band $\mathrm{H} \alpha$ imaging and compare the global $\mathrm{H} \alpha$-derived SFRs to those in the other bands compiled for the SFRS. All the related photometry will be made public to facilitate investigations by others in the community.

The authors gratefully acknowledge the assistance of R. Brent Tully, who generously supplied quality distances for many of our sample galaxies. This research has made use of the NASA/IPAC Extragalactic Database (NED) which is operated by the Jet Propulsion Laboratory, California Institute of Technology, under contract with the National Aeronautics and Space Administration. This work is based in part on observations made with the Spitzer Space Telescope, which is operated by the Jet Propulsion Laboratory, California Institute of Technology under a contract with NASA. Support for this work was provided by NASA. The National Radio Astronomy Observatory is a facility of the National Science Foundation operated under cooperative agreement by Associated Universities, Inc. $\mathrm{H}$. Wu is supported by NSFC grants 10833006, 10773014, and the 973 Program grant 2007CB815406. P. Barmby acknowledges research support through a Discovery Grant from the Natural Sciences and Engineering Research Council of Canada. S. Mahajan acknowledges support from a Smithsonian Institution Endowment Grant. A. Zezas and P. Bonfini acknowledge support from Marie-Curie IRG grant 224878 and European Union grant 206469. H. Smith acknowledges partial support from NASA grants NNX07AH49G and NNX10AD83G.

Facilities: VLA, Spitzer(IRAC, MIPS), GALEX, 2MASS, SDSS, NAOC.

\section{REFERENCES}


Baldwin, J. A., Phillips, M. M., \& Terlevich, R. 1981, PASP, 93, 5 Bell, E. F. 2003, ApJ, 586, 794

Bertin, E., \& Arnouts, S. 1996, A\&AS, 117, 393

Blanton, M. R., et al. 2001, AJ, 121, 2358

Bloom, J. S., Starr, D. L., Blake, C. H., Skrutskie, M. F., \& Falco, E. E. 2006, Astronomical Data Analysis Software and Systems XV, 351, 751

Bonatto, C. J., \& Pastoriza, M. G. 1997, ApJ, 486, 132

Boselli, A., et al. 2010, PASP, 122, 261

Buat, V., et al. 2005, ApJ, 619, L51

Buat, V., et al. 2010, MNRAS, 409, L1

Calzetti, D., et al. 2010, ApJ, 714, 1256

Calzetti, D. 2001, PASP, 113, 1449

Condon, J. J. 1992, ARA\&A, 30, 575

Condon, J. J., Cotton, W. D., Greisen, E. W., Yin, Q. F., Perley, R. A., Taylor, G. B., \& Broderick, J. J. 1998, AJ, 115, 1693

Condon, J. J., Cotton, W. D., Greisen, E. W., Yin, Q. F., Perley, R. A., Taylor, G. B., \& Broderick, J. J. 2002, VizieR Online Data Catalog, 8065, 0

Dale, D. A., \& Helou, G. 2002, ApJ, 576, 159

Dale, D. A., et al. 2005, ApJ, 633, 857

Dale, D. A., et al. 2007, ApJ, 655, 863

Dale, D. A., et al. 2009, ApJ, 703, 517

Devereux, N. A., \& Eales, S. A. 1989, ApJ, 340, 708

Elbaz, D., et al. 2011, arXiv:1105.2537

Fabricant, D., Cheimets, P., Caldwell, N., \& Geary, J. 1998, PASP, 110, 79

Fazio, G. G., et al. 2004, ApJS, 154, 10

Freedman, W. L., et al. 2001, ApJ, 553, 47

Impey, C. D., \& Neugebauer, G. 1988, AJ, 95, 307

Helou, G., Soifer, B. T., \& Rowan-Robinson, M. 1985, ApJ, 298, L7

Hogg, D. W., Tremonti, C. A., Blanton, M. R., Finkbeiner, D. P., Padmanabhan, N., Quintero, A. D., Schlegel, D. J., \& Wherry, N. 2005, ApJ, 624, 162

Hopkins, A. M., \& Beacom, J. F. 2006, ApJ, 651, 142

Ishihara, D. et al. 2010, A\&A, 514, A1

Jeong, W.-S., et al. 2007, PASJ, 59, 429

Jester, S. et al. 2005, AJ, 130, 873

Karachentsev, I. D., Mitronova, S. N., Karachentseva, V. E., Kudrya, Y. N., \& Jarrett, T. H. 2002, A\&A, 396, 431

Karachentsev, I. D., Karachentseva, V. E., Huchtmeier, W. K., \& Makarov, D. I. 2004, AJ, 127, 2031

Karachentsev, I. D., Kudrya, Y. N., Karachentseva, V. E., \& Mitronova, S. N. 2006, Astrophysics, 49, 450

Kartaltepe, J. S., et al. 2010, ApJ, 709, 572

Kauffmann, G., et al. 2003, MNRAS, 346, 1055

Kawada, M., et al. 2007, PASJ, 59, S389

Kennicutt, R. C., Jr. 1998, ARA\&A, 36, 189

Kennicutt, R. C., Jr., et al. 2003, PASP, 115, 928

Kewley, L. J., Dopita, M. A., Sutherland, R. S., Heisler, C. A., \& Trevena, J. 2001, ApJ, 556, 121

Kewley, L. J., Geller, M. J., Jansen, R. A., \& Dopita, M. A. 2002, AJ, 124,3135

Kewley, L. J., Groves, B., Kauffmann, G., \& Heckman, T. 2006, MNRAS, 372, 961

Kim, D.-C., Veilleux, S., \& Sanders, D. B. 2002, ApJS, 143, 277

Kirby, E. M., Jerjen, H., Ryder, S. D., \& Driver, S. P. 2008, AJ, 136,1866
Laag, E., Croft, S., Canalizo, G., \& Lacy, M. 2010, arXiv: 1010.1704

Lada, C. J., Lombardi, M., \& Alves, J. F. 2010, arXiv:1009.2985

Lamarre, J.-M., et al. 2010, A\&A, 520, A9

Lilly, S. J., Le Fevre, O., Hammer, F., \& Crampton, D. 1996, ApJ, 460, L1

Lupton, R., Gunn, J. E., Ivezić, Z., Knapp, G. R., \& Kent, S. 2001, Astronomical Data Analysis Software and Systems X, 238,269

Madden, S. C., Galliano, F., Jones, A. P., \& Sauvage, M. 2006, A\&A, 446, 877

Madau, P., Pozzetti, L., \& Dickinson, M. 1998, ApJ, 498, 106

Martin, D. C., et al. 2005, ApJ, 619, L1

Mould, J. R., et al. 2000, ApJ, 529, 786

Mullaney, J. R., Alexander, D. M., Goulding, A. D., \& Hickox, R. C. 2011, MNRAS, 414, 1082

Murakami, H., et al. 2007, PASJ, 59, S369

Netzer, H., et al. 2007, ApJ, 666, 806

Oconnell, R. W., Thuan, T. X., \& Goldstein, S. J. 1978, ApJ, 226, L11

Parker, N. D., 1991, MNRAS, 251, 63

Poggianti, B. M., \& Wu, H. 2000, ApJ, 529, 157

Rice, W., et al. 1988, ApJS, 68, 91

Rieke, G. H., Lebofsky, M. J., Thompson, R. I., Low, F. J., \& Tokunaga, A. T. 1980, ApJ, 238, 24

Rieke, G. H., et al. 2004, ApJS, 154, 25

Sanders, D. B., Soifer, B. T., Elias, J. H., Neugebauer, G., \& Matthews, K. 1988, ApJ, 328, L35

Sanders, D. B., Mazzarella, J. M., Kim, D.-C., Surace, J. A., \& Soifer, B. T. 2003, AJ, 126, 1607

Saunders, W., et al. 2000, MNRAS, 317, 55

Schuster, M. T., Marengo, M., \& Patten, B. M. 2006, Proc. SPIE, 6270,65

Sobral, D., Best, P. N., Smail, I., Geach, J. E., Cirasuolo, M., Garn, T., \& Dalton, G. B. 2011, MNRAS, 411, 675

Stern, D., et al. 2005, ApJ, 631, 163

Planck Collaboration 2011a, arXiv:1101.2022

Planck Collaboration 2011b, The Early Release Compact Source Catalog. arXiv:1101.2041

Planck Collaboration 2011c, arXiv:1101.2045

Schmidt, M. 1959, ApJ, 129, 243

Takeuchi, T. T., Buat, V., Heinis, S., Giovannoli, E., Yuan, F.-T. Iglesias-Páramo, J., Murata, K. L., \& Burgarella, D. 2010, A\&A, 514, A4

Tonry, J. L., Dressler, A., Blakeslee, J. P., Ajhar, E. A., Fletcher, A. B., Luppino, G. A., Metzger, M. R., \& Moore, C. B. 2001, ApJ, 546, 681

Tully, R. B., \& Fisher, J. R. 1977, A\&A, 54, 661

Tully, R. B., Shaya, E. J., Karachentsev, I. D., Courtois, H. M., Kocevski, D. D., Rizzi, L., \& Peel, A. 2008, ApJ, 676, 184

Wu, H., Cao, C., Hao, C.-N., Liu, F.-S., Wang, J.-L., Xia, X.-Y., Deng, Z.-G., \& Young, C. K.-S. 2005, ApJ, 632, L79

Wuyts, S., et al. 2011, arXiv:1106.5502

Yun, M. S., Reddy, N. A., \& Condon, J. J. 2001, ApJ, 554, 803 
The Star Formation Reference Survey

TABLE 2

Basic Galaxy Parameters for SFRS Sample Galaxies

\begin{tabular}{|c|c|c|c|c|c|c|c|c|c|c|c|}
\hline SFRS & Name & $\begin{array}{c}\text { Posi } \\
\text { (J2 }\end{array}$ & $\begin{array}{l}\mathrm{on}^{\mathrm{a}} \\
00)\end{array}$ & $\begin{array}{c}\text { Distance }^{b} \\
\text { (Mpc) }\end{array}$ & $\begin{array}{c}\log L_{60} \\
\left(\mathrm{~L}_{\odot}\right)\end{array}$ & $F_{100} / F_{60}$ & $\begin{array}{l}K_{s}-F_{60} \\
(\mathrm{AB} \mathrm{mag})\end{array}$ & Weight & $\begin{array}{l}\text { Axial } \\
\text { Ratio }\end{array}$ & $\begin{array}{c}\mathrm{T} \\
\text { Type }\end{array}$ & $\begin{array}{c}\text { Extinction } \\
\text { ( } B \text { mag })\end{array}$ \\
\hline 1 & IC 486 & $8: 00: 20.98$ & $26: 36: 48.7$ & 114.4 & 9.55 & 1.53 & 3.51 & 1.5 & 0.76 & 1 & 0.20 \\
\hline 2 & IC 2217 & 8:00:49.73 & $27: 30: 01.7$ & 76.1 & 9.57 & 1.96 & 4.92 & 13.2 & 0.81 & 20 & 0.25 \\
\hline 3 & NGC 2500 & $8: 01: 53.18$ & $50: 44: 13.7$ & 15.0 & 7.93 & 2.05 & 4.03 & 4.2 & 0.86 & 7 & 0.23 \\
\hline 4 & NGC 2512 & $8: 03: 07.85$ & $23: 23: 30.6$ & 69.3 & 9.67 & 1.92 & 4.31 & 7.4 & 0.76 & 3 & 0.30 \\
\hline 5 & MCG 6-18-009 & $8: 03: 28.94$ & $33: 27: 44.5$ & 164.3 & 10.11 & 1.87 & 4.45 & 1.5 & 0.83 & $\ldots$ & 0.27 \\
\hline 6 & MK 1212 & $8: 07: 05.52$ & $27: 07: 33.7$ & 173.3 & 10.21 & 1.80 & 5.33 & 7.4 & 0.94 & $\cdots$ & 0.25 \\
\hline 7 & IRAS $08072+1847$ & $8: 10: 15.17$ & $33: 57: 23.9$ & 70.8 & 9.55 & 1.10 & 6.37 & 8.1 & 0.98 & $\cdots$ & 0.23 \\
\hline 8 & NGC 2532 & $8: 10: 07.01$ & $18: 38: 18.1$ & 77.6 & 9.75 & 2.86 & 4.13 & 5.0 & 0.87 & 5 & 0.30 \\
\hline 9 & UGC 4261 & $8: 10: 56.21$ & $36: 49: 41.3$ & 93.2 & 9.45 & 1.28 & 5.39 & 4.3 & 0.71 & 15 & 0.29 \\
\hline 10 & NGC 2535 & $8: 11: 13.08$ & $25: 12: 15.3$ & 61.6 & 9.30 & 2.80 & 4.16 & 5.5 & 0.56 & 5 & 0.26 \\
\hline 11 & NGC 2543 & $8: 12: 57.91$ & $36: 15: 16.7$ & $26.3 \pm 5.5$ & 9.03 & 2.21 & 3.68 & 12.1 & 0.57 & 3 & 0.31 \\
\hline 12 & NGC 2537 & $8: 13: 14.74$ & $45: 59: 21.9$ & 15.0 & 7.82 & 1.85 & 3.52 & 3.2 & 0.74 & 9 & 0.23 \\
\hline 13 & IC 2233 & $8: 13: 58.82$ & $45: 44: 43.7$ & $13.7 \pm 2.8$ & 7.47 & 1.58 & 4.14 & 1.0 & 0.16 & 7 & 0.23 \\
\hline 14 & IC 2239 & $8: 14: 06.79$ & $23: 51: 58.9$ & 88.5 & 9.78 & 1.80 & 4.56 & 13.2 & 0.84 & $\cdots$ & 0.27 \\
\hline 15 & UGC 4286 & $8: 14: 16.50$ & $18: 26: 26.0$ & 73.5 & 9.10 & 2.08 & 6.48 & 3.0 & 0.41 & 3 & 0.26 \\
\hline 16 & UGC 4306 & $8: 17: 36.60$ & $35: 26: 50.7$ & 36.0 & 9.12 & 1.89 & 4.74 & 8.3 & 0.64 & 20 & 0.33 \\
\hline 17 & NGC 2552 & $8: 19: 19.58$ & 50:00:20.8 & $11.4 \pm 0.8$ & 7.25 & 2.46 & 6.01 & 1.0 & 0.67 & 9 & 0.21 \\
\hline 18 & IC 2339 & $8: 23: 34.20$ & $21: 20: 51.5$ & 79.3 & 9.43 & 1.75 & 5.63 & 3.0 & 0.52 & 5 & 0.25 \\
\hline 19 & IRAS $08234+1054 \mathrm{~B}$ & $8: 26: 07.90$ & $10: 44: 51.3$ & 272.6 & 10.61 & 1.75 & 6.61 & 6.6 & 0.58 & $\ldots$ & 0.26 \\
\hline 20 & IRAS $08269+1514$ & $8: 29: 45.19$ & $15: 04: 39.4$ & 134.5 & 9.60 & 2.87 & 5.18 & 3.0 & 0.46 & $\cdots$ & 0.24 \\
\hline 21 & NGC 2604 & $8: 33: 23.14$ & $29: 32: 19.7$ & $36.3 \pm 4.2$ & 8.57 & 2.18 & 4.70 & 4.7 & 0.92 & 6 & 0.26 \\
\hline 22 & NGC 2608 & $8: 35: 39.96$ & $46: 29: 28.1$ & $36.3 \pm 4.2$ & 8.83 & 2.60 & 3.37 & 12.1 & 0.66 & 3 & 0.26 \\
\hline 23 & MK 92 & $8: 35: 17.06$ & $28: 28: 30.9$ & 68.8 & 9.48 & 1.53 & 5.45 & 4.3 & 0.79 & $\ldots$ & 0.17 \\
\hline 24 & NGC 2623 & $8: 38: 24.00$ & $25: 45: 16.3$ & 81.6 & 10.62 & 1.16 & 6.95 & 15.5 & 0.45 & $\ldots$ & 0.20 \\
\hline 25 & CGCG 120-018 & $8: 39: 50.76$ & 23:08:36.1 & 107.9 & 9.99 & 1.42 & 5.80 & 8.1 & 0.99 & $\cdots$ & 0.18 \\
\hline 26 & NGC 2644 & $8: 41: 31.85$ & 4:58:49.2 & 25.0 & 8.49 & 2.37 & 3.91 & 4.6 & 0.44 & 15 & 0.22 \\
\hline 27 & UGC 4572 & $8: 45: 37.85$ & $36: 56: 04.7$ & 60.5 & 8.95 & 2.07 & 3.69 & 4.3 & 0.88 & 11 & 0.21 \\
\hline 28 & UGC 4653 & $8: 53: 54.62$ & $35: 08: 44.2$ & 234.0 & 10.54 & 2.13 & 4.51 & 1.0 & 0.70 & 3 & 0.19 \\
\hline 29 & IRAS $08512+2727$ & $8: 54: 16.78$ & $27: 15: 59.5$ & 265.3 & 10.15 & 2.70 & 4.48 & 1.0 & 0.87 & $\ldots$ & 0.23 \\
\hline 30 & OJ 287 & $8: 54: 48.86$ & $20: 06: 30.7$ & 1258.8 & 11.75 & 1.45 & 4.46 & 1.0 & 1.00 & $\cdots$ & 0.13 \\
\hline 31 & IRAS $08538+4256$ & $8: 57: 10.32$ & $42: 45: 23.1$ & 121.2 & 10.27 & 1.38 & 6.36 & 14.2 & 0.88 & $\cdots$ & 0.15 \\
\hline 32 & IRAS $08550+3908$ & $8: 58: 13.75$ & $38: 56: 31.9$ & 367.8 & 10.72 & 1.25 & 5.19 & 1.5 & 0.55 & $\cdots$ & 0.14 \\
\hline 33 & NGC 2718 & $8: 58: 50.47$ & $6: 17: 34.8$ & 57.4 & 9.50 & 1.74 & 4.13 & 9.5 & 0.85 & 2 & 0.31 \\
\hline 34 & NGC 2712 & $8: 59: 30.48$ & $44: 54: 50.0$ & $30.9 \pm 6.4$ & 8.72 & 2.42 & 3.08 & 12.1 & 0.54 & 3 & 0.14 \\
\hline 35 & NGC 2719 & $9: 00: 15.72$ & $35: 43: 39.5$ & 51.1 & 9.05 & 1.41 & 6.90 & 4.3 & 0.32 & 10 & 0.18 \\
\hline 36 & IRAS $08572+3915$ & $9: 00: 25.37$ & $39: 03: 53.7$ & 244.3 & 11.13 & 0.61 & 8.31 & 15.5 & 0.89 & $\ldots$ & 0.14 \\
\hline 37 & IRAS $08579+3447$ & $9: 01: 05.78$ & $34: 35: 28.6$ & 273.5 & 10.80 & 1.73 & 6.14 & 6.6 & 0.44 & $\cdots$ & 0.16 \\
\hline 38 & NGC 2731 & $9: 02: 08.40$ & $8: 18: 06.0$ & 35.0 & 9.04 & 1.67 & 4.81 & 6.9 & 0.72 & 20 & 0.34 \\
\hline 39 & NGC 2730 & $9: 02: 15.82$ & $16: 50: 17.9$ & 58.9 & 8.79 & 2.97 & 4.56 & 1.5 & 0.73 & 8 & 0.21 \\
\hline 40 & IC 2431 & $9: 04: 34.39$ & $14: 35: 39.4$ & 209.0 & 10.75 & 1.72 & 5.92 & 6.6 & 0.53 & 17 & 0.23 \\
\hline 41 & NGC 2750 & $9: 05: 47.93$ & $25: 26: 15.0$ & 37.0 & 9.22 & 1.95 & 4.58 & 8.3 & 0.72 & 5 & 0.19 \\
\hline 42 & IC 2434 & $9: 07: 16.06$ & $37: 12: 55.3$ & 104.5 & 9.69 & 2.02 & 3.89 & 7.4 & 0.59 & 20 & 0.13 \\
\hline 43 & NGC 2761 & $9: 07: 30.84$ & $18: 26: 05.1$ & 125.0 & 10.22 & 1.94 & 5.25 & 7.4 & 0.64 & $\ldots$ & 0.20 \\
\hline 44 & NGC 2773 & $9: 09: 44.16$ & $7: 10: 25.7$ & 80.4 & 9.66 & 1.85 & 4.63 & 13.2 & 0.74 & $\cdots$ & 0.30 \\
\hline 45 & NGC 2776 & $9: 12: 14.52$ & $44: 57: 17.4$ & 36.0 & 9.18 & 2.68 & 3.63 & 12.1 & 0.86 & 5 & 0.09 \\
\hline 46 & NGC 2789 & $9: 14: 59.66$ & $29: 43: 48.9$ & 93.6 & 9.70 & 2.31 & 3.76 & 3.8 & 0.77 & 0 & 0.15 \\
\hline 47 & IRAS $09121+3908$ & $9: 15: 22.15$ & $38: 56: 35.0$ & 42.5 & 10.61 & 2.24 & 6.08 & 3.5 & 0.77 & $\cdots$ & 0.08 \\
\hline 48 & NGC 2824 & $9: 19: 02.23$ & $26: 16: 11.9$ & 37.0 & 8.70 & 1.63 & 3.26 & 2.3 & 0.63 & -2 & 0.21 \\
\hline 49 & IRAS $09184+4356$ & $9: 21: 38.74$ & $43: 43: 34.1$ & 170.1 & 10.03 & 2.14 & 5.09 & 4.7 & 0.58 & $\ldots$ & 0.09 \\
\hline 50 & CGCG 238-041 & $9: 22: 25.30$ & $47: 14: 39.9$ & 131.5 & 9.45 & 3.28 & 5.48 & 1.0 & 0.76 & $\cdots$ & 0.08 \\
\hline 51 & UGC 4985 & $9: 22: 37.39$ & $21: 57: 26.8$ & 143.4 & 9.68 & 2.79 & 4.65 & 3.0 & 0.43 & $\cdots$ & 0.19 \\
\hline 52 & NGC 2854 & $9: 24: 02.83$ & 49:12:13.7 & 25.0 & 8.99 & 1.47 & 3.86 & 5.7 & 0.51 & 3 & 0.10 \\
\hline 53 & UGC 5046 & $9: 28: 06.65$ & $17: 11: 47.4$ & 64.9 & 9.48 & 1.69 & 4.82 & 6.9 & 0.53 & 20 & 0.14 \\
\hline 54 & UGC 5055 & $9: 30: 11.76$ & $55: 51: 08.7$ & 110.6 & 9.70 & 2.05 & 4.34 & 7.4 & 0.85 & 3 & 0.14 \\
\hline 55 & NGC 2893 & $9: 30: 16.97$ & $29: 32: 23.9$ & 24.0 & 8.72 & 1.51 & 4.33 & 5.7 & 0.90 & 1 & 0.12 \\
\hline 56 & MCG 3-24-062 & $9: 30: 22.99$ & 19:28:09.3 & 66.2 & 9.11 & 2.81 & 4.10 & 5.5 & 0.69 & $\cdots$ & 0.17 \\
\hline 57 & CGCG 238-066 & $9: 31: 06.77$ & 49:04:47.1 & 147.0 & 10.02 & 1.35 & 5.05 & 6.0 & 0.80 & $\cdots$ & 0.09 \\
\hline 58 & UGC 5097 & $9: 34: 10.63$ & $0: 14: 31.9$ & 72.5 & 9.55 & 1.70 & 5.34 & 8.1 & 0.71 & 15 & 0.34 \\
\hline 59 & CGCG 289-012 & $9: 36: 31.87$ & $59: 23: 54.3$ & 172.4 & 10.10 & 2.17 & 7.42 & 4.5 & 0.89 & $\ldots$ & 0.14 \\
\hline 60 & MCG 8-18-013 & $9: 36: 37.18$ & $48: 28: 28.0$ & 110.9 & 10.28 & 1.43 & 5.99 & 14.2 & 0.68 & $\cdots$ & 0.09 \\
\hline 61 & CGCG 181-068 & $9: 37: 19.22$ & $33: 49: 25.8$ & 100.6 & 9.49 & 2.74 & 4.19 & 5.5 & 0.39 & $\cdots$ & 0.07 \\
\hline 62 & NGC 2936 & $9: 37: 44.14$ & $2: 45: 39.0$ & 100.5 & 9.74 & 2.34 & 3.78 & 8.7 & 0.52 & -5 & 0.27 \\
\hline 63 & NGC 2955 & $9: 41: 16.61$ & $35: 52: 56.2$ & 103.5 & 9.65 & 2.80 & 3.63 & 4.8 & 0.55 & 3 & 0.06 \\
\hline 64 & CGCG $182-010$ & $9: 45: 15.22$ & $34: 42: 44.2$ & 175.1 & 10.22 & 1.76 & 5.11 & 7.4 & 0.72 & $\cdots$ & 0.07 \\
\hline 65 & UGC 5228 & $9: 46: 03.60$ & 1:40:06.1 & $28.2 \pm 4.0$ & 8.49 & 2.26 & 3.91 & 4.6 & 0.29 & 5 & 0.54 \\
\hline 66 & IRAS $09438+1141$ & $9: 46: 32.57$ & $11: 27: 19.5$ & 203.0 & 10.13 & 2.49 & 5.63 & 4.5 & 0.87 & $\cdots$ & 0.21 \\
\hline 67 & NGC 3015 & $9: 49: 22.92$ & 1:08:43.5 & 108.8 & 9.81 & 1.98 & 4.37 & 7.4 & 0.79 & -2 & 0.39 \\
\hline 68 & MCG 2-25-039 & $9: 49: 36.98$ & $9: 00: 18.8$ & 77.6 & 9.32 & 2.23 & 4.72 & 4.7 & 0.44 & $\ldots$ & 0.20 \\
\hline 69 & NGC 3020 & $9: 50: 06.65$ & $12: 48: 49.0$ & $18.3 \pm 2.6$ & 8.35 & 1.71 & 4.95 & 3.0 & 0.43 & 6 & 0.36 \\
\hline 70 & NGC 3049 & $9: 54: 49.56$ & $9: 16: 15.9$ & $18.3 \pm 2.6$ & 8.52 & 1.60 & 4.43 & 5.7 & 0.65 & 2 & 0.23 \\
\hline 71 & NGC 3055 & $9: 55: 18.07$ & 4:16:12.1 & 25.0 & 8.87 & 2.09 & 4.14 & 9.5 & 0.56 & 5 & 0.31 \\
\hline 72 & IC 2520 & $9: 56: 20.11$ & $27: 13: 39.3$ & $26.4 \pm 1.6$ & 8.63 & 1.98 & 4.73 & 8.3 & 0.82 & 15 & 0.09 \\
\hline 73 & UGC 5403 & 10:02:35.54 & 19:10:36.9 & 33.0 & 8.85 & 1.58 & 4.45 & 5.7 & 0.54 & 0 & 0.15 \\
\hline
\end{tabular}


TABLE $2-$ Continued

\begin{tabular}{|c|c|c|c|c|c|c|c|c|c|c|c|}
\hline SFRS & Name & $\begin{array}{r}\text { Posi } \\
\text { (J2 }\end{array}$ & $\begin{array}{l}\text { on }^{\mathrm{a}} \\
00)\end{array}$ & $\begin{array}{l}\text { Distance }^{\mathrm{b}} \\
(\mathrm{Mpc})\end{array}$ & $\begin{array}{c}\log L_{60} \\
\left(\mathrm{~L}_{\odot}\right)\end{array}$ & $F_{100} / F_{60}$ & $\begin{array}{c}K_{s}-F_{60} \\
(\mathrm{AB} \text { mag) }\end{array}$ & Weight & $\begin{array}{l}\text { Axial } \\
\text { Ratio }\end{array}$ & $\begin{array}{c}\mathrm{T} \\
\text { Type }\end{array}$ & $\begin{array}{c}\text { Extinction } \\
(B \text { mag })\end{array}$ \\
\hline 74 & UGC 5459 & 10:08:10.08 & $53: 05: 01.5$ & $25.8 \pm 1.7$ & 8.40 & 2.30 & 3.71 & 5.0 & 0.20 & 5 & 0.07 \\
\hline 75 & MCG 5-24-022 & 10:10:03.38 & $32: 04: 12.9$ & 92.1 & 9.31 & 2.68 & 6.88 & 2.3 & 0.67 & $\ldots$ & 0.17 \\
\hline 76 & IC 2551 & $10: 10: 40.32$ & $24: 24: 50.9$ & 94.9 & 9.94 & 1.10 & 5.28 & 9.8 & 0.79 & $\ldots$ & 0.20 \\
\hline 77 & IRAS $10106+2745$ & $10: 13: 29.50$ & $27: 30: 40.1$ & 215.6 & 10.03 & 2.73 & 4.77 & 2.7 & 0.55 & $\ldots$ & 0.18 \\
\hline 78 & NGC 3162 & $10: 13: 31.58$ & $22: 44: 15.2$ & $26.4 \pm 1.6$ & 8.52 & 2.21 & 3.86 & 14.5 & 0.82 & 4 & 0.15 \\
\hline 79 & IRAS $10120+1653$ & $10: 14: 47.90$ & $16: 38: 50.1$ & 517.2 & 10.89 & 2.19 & 6.51 & 3.5 & 0.71 & $\ldots$ & 0.24 \\
\hline 80 & NGC 3190 & 10:18:05.66 & $21: 49: 56.1$ & $26.4 \pm 1.6$ & 8.59 & 2.95 & 1.80 & 22.2 & 0.37 & 1 & 0.20 \\
\hline 81 & IC 602 & $10: 18: 19.73$ & $7: 02: 57.5$ & 57.6 & 9.32 & 2.01 & 4.75 & 8.3 & 0.63 & 20 & 0.18 \\
\hline 82 & NGC 3191 & $10: 19: 05.14$ & $46: 27: 14.8$ & 134.0 & 9.91 & 2.24 & 4.79 & 8.7 & 0.79 & 4 & 0.08 \\
\hline 83 & NGC 3206 & $10: 21: 47.59$ & $56: 55: 49.5$ & $25.8 \pm 1.7$ & 8.13 & 2.00 & 4.61 & 2.8 & 0.71 & 6 & 0.09 \\
\hline 84 & UGC 5613 & $10: 23: 32.54$ & $52: 20: 31.3$ & 139.8 & 10.35 & 1.97 & 5.40 & 8.4 & 0.72 & 8 & 0.06 \\
\hline 85 & UGC 5644 & $10: 25: 46.25$ & 13:43:00.7 & 137.6 & 9.52 & 2.03 & 3.26 & 1.7 & 0.86 & 20 & 0.22 \\
\hline 86 & NGC 3245 & $10: 27: 18.41$ & $28: 30: 26.6$ & $20.9 \pm 2.1$ & 8.49 & 1.60 & 1.62 & 2.0 & 0.53 & -2 & 0.16 \\
\hline 87 & IRAS 10246+2042 & $10: 27: 25.87$ & $20: 26: 51.4$ & 84.2 & 9.64 & 1.50 & 5.07 & 9.8 & 0.87 & . & 0.19 \\
\hline 88 & MCG 7-22-012 & 10:30:11.42 & $43: 21: 38.1$ & 66.0 & 9.16 & 2.63 & 7.19 & 2.3 & 0.45 & $\ldots$ & 0.09 \\
\hline 89 & IRAS $10276+1119$ & $10: 30: 14.76$ & $11: 04: 15.9$ & 271.3 & 10.23 & 3.82 & 5.05 & 2.7 & 0.78 & $\ldots$ & 0.16 \\
\hline 90 & NGC 3265 & $10: 31: 06.77$ & $28: 47: 48.0$ & $21.8 \pm 1.6$ & 8.58 & 1.54 & 4.33 & 5.7 & 0.82 & -5 & 0.14 \\
\hline 91 & UGC 5713 & $10: 31: 38.90$ & $25: 59: 02.1$ & 95.0 & 9.31 & 1.24 & 3.49 & 2.3 & 0.36 & 4 & 0.16 \\
\hline 92 & NGC 3274 & $10: 32: 31.87$ & $54: 24: 03.7$ & 10.0 & 7.04 & 1.88 & 4.05 & 1.7 & 0.58 & 7 & 0.16 \\
\hline 93 & UGC 5720 & $10: 32: 17.23$ & $27: 40: 07.7$ & 20.0 & 8.94 & 1.15 & 5.35 & 4.3 & 0.66 & 10 & 0.09 \\
\hline 94 & KUG 1031+351 & $10: 34: 02.40$ & $34: 52: 10.2$ & 298.2 & 10.86 & 1.87 & 5.29 & 1.5 & 0.95 & $\ldots$ & 0.10 \\
\hline 95 & NGC 3306 & $10: 37: 10.22$ & $12: 39: 09.3$ & $46.6 \pm 8.9$ & 9.11 & 1.82 & 4.59 & 8.3 & 0.66 & 9 & 0.16 \\
\hline 96 & NGC 3323 & 10:39:39.02 & $25: 19: 21.9$ & 79.5 & 9.35 & 2.20 & 4.92 & 4.7 & 0.64 & 20 & 0.19 \\
\hline 97 & IC 2598 & $10: 39: 42.38$ & $26: 43: 38.6$ & 89.1 & 9.80 & 1.69 & 5.31 & 9.8 & 0.73 & 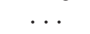 & 0.16 \\
\hline 98 & NGC 3338 & $10: 42: 07.54$ & $13: 44: 49.2$ & $21.4 \pm 4.0$ & 8.50 & 3.01 & 2.73 & 13.2 & 0.49 & 5 & 0.19 \\
\hline 99 & NGC 3353 & $10: 45: 22.06$ & $55: 57: 39.9$ & 16.0 & 8.67 & 1.28 & 5.55 & 4.3 & 0.73 & 3 & 0.08 \\
\hline 100 & UGC 5881 & $10: 46: 42.53$ & $25: 55: 53.6$ & 93.0 & 9.57 & 1.39 & 4.41 & 4.8 & 0.54 & 1 & 0.20 \\
\hline 101 & NGC 3370 & $10: 47: 04.06$ & $17: 16: 25.0$ & $20.9 \pm 1.2$ & 8.57 & 2.66 & 3.82 & 14.5 & 0.56 & 5 & 0.18 \\
\hline 102 & NGC 3381 & $10: 48: 24.82$ & $34: 42: 41.1$ & $25.7 \pm 5.3$ & 8.48 & 2.82 & 4.15 & 2.3 & 0.87 & 20 & 0.11 \\
\hline 103 & UGC 5941 & $10: 50: 21.60$ & $41: 27: 50.5$ & 107.0 & 9.87 & 1.89 & 4.78 & 13.2 & 0.88 & 17 & 0.06 \\
\hline 104 & NGC 3413 & 10:51:20.74 & $32: 45: 59.0$ & $16.2 \pm 1.6$ & 7.27 & 1.90 & 3.87 & 1.7 & 0.59 & -2 & 0.14 \\
\hline 105 & NGC 3408 & 10:52:11.69 & $58: 26: 17.3$ & 138.0 & 9.67 & 1.70 & 3.76 & 1.5 & 0.79 & 5 & 0.08 \\
\hline 106 & NGC 3430 & $10: 52: 11.40$ & $32: 57: 01.5$ & 28.4 & 8.78 & 2.90 & 3.11 & 22.2 & 0.83 & 5 & 0.11 \\
\hline 107 & CGCG 95-055 & $10: 52: 50.74$ & $16: 59: 07.6$ & $25.7 \pm 5.3$ & 10.52 & 0.91 & 5.32 & 1.5 & 0.53 & 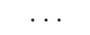 & 0.20 \\
\hline 108 & IRAS $10565+2448$ & $10: 59: 18.12$ & $24: 32: 34.7$ & 185.2 & 11.06 & 1.18 & 7.08 & 15.5 & 0.88 & 10 & 0.10 \\
\hline 109 & UGC 6074 & $10: 59: 58.25$ & $50: 54: 10.6$ & $38.0 \pm 7.1$ & 9.28 & 1.34 & 5.03 & 6.9 & 0.79 & 15 & 0.09 \\
\hline 110 & NGC 3495 & $11: 01: 16.22$ & $3: 37: 40.7$ & $17.5 \pm 2.3$ & 8.41 & 2.62 & 3.19 & 5.0 & 0.25 & 7 & 0.27 \\
\hline 111 & UGC 6103 & 11:01:58.99 & $45: 13: 40.9$ & 91.7 & 9.71 & 1.67 & 4.77 & 9.8 & 0.78 & $\cdots$ & 0.06 \\
\hline 112 & MCG 7-23-019 & 11:03:54.31 & 40:51:00.1 & 150.6 & 10.57 & 1.76 & 6.41 & 6.6 & 0.70 & 10 & 0.09 \\
\hline 113 & UGC 6135 & 11:04:36.96 & $45: 07: 30.8$ & 90.9 & 9.55 & 2.91 & 3.63 & 4.8 & 0.93 & 20 & 0.06 \\
\hline 114 & CGCG 241-078 & $11: 06: 37.37$ & $46: 02: 19.6$ & 110.9 & 9.88 & 1.40 & 5.54 & 8.1 & 0.56 & $\ldots$ & 0.06 \\
\hline 115 & IRAS $11069+2711$ & 11:09:39.36 & $26: 54: 53.9$ & 296.4 & 10.75 & 1.95 & 6.33 & 6.6 & 0.49 & $\ldots$ & 0.13 \\
\hline 116 & IC 676 & $11: 12: 39.82$ & 9:03:21.0 & $26.9 \pm 2.2$ & 8.54 & 1.56 & 4.08 & 5.7 & 0.71 & -1 & 0.18 \\
\hline 117 & IRAS $11102+3026$ & $11: 12: 57.36$ & $30: 10: 28.6$ & 129.6 & 10.00 & 1.78 & 6.02 & 7.0 & 0.66 & 20 & 0.11 \\
\hline 118 & IC 2637 & $11: 13: 49.75$ & $9: 35: 10.7$ & 128.2 & 9.91 & 1.75 & 4.28 & 7.4 & 0.94 & -4 & 0.18 \\
\hline 119 & MCG 9-19-013 & $11: 14: 49.37$ & $50: 19: 22.5$ & 201.8 & 10.02 & 2.73 & 5.14 & 2.7 & 0.78 & $\cdots$ & 0.09 \\
\hline 120 & 7ZW 384 & $11: 16: 54.41$ & $59: 31: 50.8$ & 340.4 & 10.53 & 2.43 & 5.31 & 1.5 & 0.62 & $\ldots$ & 0.07 \\
\hline 121 & IRAS $11167+5351$ & $11: 19: 34.01$ & $53: 35: 18.7$ & 447.3 & 10.96 & 1.76 & 5.54 & 6.6 & 0.90 & $\ldots$ & 0.08 \\
\hline 122 & NGC 3633 & $11: 20: 26.21$ & $3: 35: 08.2$ & 30.0 & 9.05 & 1.76 & 4.05 & 9.5 & 0.49 & 1 & 0.30 \\
\hline 123 & NGC 3652 & $11: 22: 39.02$ & $37: 45: 54.4$ & $15.5 \pm 3.2$ & 8.86 & 2.53 & 4.83 & 4.7 & 0.46 & 6 & 0.13 \\
\hline 124 & NGC 3656 & $11: 23: 38.64$ & $53: 50: 31.7$ & 37.0 & 9.12 & 2.28 & 3.27 & 12.1 & 0.84 & 90 & 0.09 \\
\hline 125 & NGC 3659 & $11: 23: 45.53$ & $17: 49: 07.2$ & $21.0 \pm 1.2$ & 8.26 & 2.39 & 3.94 & 4.6 & 0.55 & 9 & 0.18 \\
\hline 126 & NGC 3664 & $11: 24: 24.26$ & $3: 19: 31.0$ & $26.9 \pm 2.2$ & 7.99 & 2.06 & 5.41 & 2.0 & 0.92 & 9 & 0.27 \\
\hline 127 & NGC 3666 & $11: 24: 26.06$ & $11: 20: 32.0$ & $16.3 \pm 1.7$ & 8.41 & 2.94 & 3.42 & 13.2 & 0.29 & 5 & 0.25 \\
\hline 128 & IC 691 & $11: 26: 44.30$ & 59:09:19.5 & 16.0 & 8.70 & 1.33 & 5.34 & 6.9 & 0.75 & 11 & 0.07 \\
\hline 129 & NGC 3686 & $11: 27: 43.97$ & $17: 13: 27.0$ & $21.0 \pm 1.2$ & 8.47 & 2.70 & 2.88 & 13.2 & 0.79 & 4 & 0.19 \\
\hline 130 & UGC 6469 & $11: 28: 17.71$ & $2: 39: 14.3$ & 102.6 & 9.62 & 1.73 & 6.14 & 7.0 & 0.44 & 4 & 0.24 \\
\hline 131 & IC 694 & $11: 28: 31.51$ & $58: 33: 51.4$ & 52.6 & 10.81 & 1.05 & 6.56 & 15.5 & 0.84 & 9 & 0.07 \\
\hline 132 & IC 698 & 11:29:03.84 & 9:06:43.4 & 96.8 & 9.92 & 1.90 & 4.61 & 13.2 & 0.61 & -2 & 0.25 \\
\hline 133 & IRAS $11267+1558$ & $11: 29: 24.70$ & $15: 41: 41.3$ & 736.6 & 11.18 & 2.72 & 6.80 & 1.5 & 0.89 & $\ldots$ & 0.22 \\
\hline 134 & NGC 3705 & 11:30:07.03 & $9: 16: 40.8$ & $16.3 \pm 1.7$ & 7.95 & 2.82 & 2.39 & 13.2 & 0.46 & 2 & 0.25 \\
\hline 135 & MCG 3-29-061 & 11:31:03.70 & $20: 14: 08.3$ & 67.5 & 9.43 & 1.53 & 5.29 & 6.9 & 0.84 & & 0.16 \\
\hline 136 & NGC 3720 & $11: 32: 21.60$ & $0: 48: 14.4$ & 89.8 & 9.68 & 2.40 & 6.58 & 4.0 & 0.88 & 1 & 0.23 \\
\hline 137 & NGC 3729 & 11:33:49.32 & $53: 07: 32.0$ & $17.1 \pm 0.8$ & 8.48 & 2.61 & 2.81 & 5.0 & 0.67 & 1 & 0.06 \\
\hline 138 & MCG 10-17-019 & $11: 35: 24.79$ & $57: 38: 59.8$ & 127.5 & 9.78 & 2.94 & 4.29 & 5.0 & 0.76 & $\ldots$ & 0.10 \\
\hline 139 & NGC 3758 & $11: 36: 28.94$ & $21: 35: 46.5$ & 131.6 & 9.80 & 1.70 & 3.87 & 4.8 & 0.91 & $\ldots$ & 0.14 \\
\hline 140 & UGC 6583 & $11: 36: 54.36$ & 19:58:18.1 & 93.2 & 9.61 & 2.22 & 4.97 & 8.7 & 0.69 & $\ldots$ & 0.16 \\
\hline 141 & MCG 1-30-003 & $11: 37: 06.58$ & $2: 50: 44.9$ & 128.9 & 9.92 & 1.75 & 5.62 & 7.0 & 0.64 & 3 & 0.20 \\
\hline 142 & NGC 3769 & 11:37:44.11 & $47: 53: 35.1$ & $17.1 \pm 0.8$ & 8.05 & 2.67 & 3.30 & 5.0 & 0.37 & 3 & 0.13 \\
\hline 143 & NGC 3773 & $11: 38: 13.06$ & $12: 06: 44.4$ & $16.3 \pm 1.7$ & 7.53 & 1.22 & 4.40 & 2.3 & 0.85 & -2 & 0.26 \\
\hline 144 & NGC 3781 & 11:39:03.77 & $26: 21: 42.2$ & 103.5 & 10.27 & 1.41 & 5.65 & 14.2 & 0.81 & .. & 0.13 \\
\hline 145 & UGC 6625 & $11: 39: 47.54$ & 19:56:00.2 & 158.2 & 9.91 & 2.73 & 4.27 & 5.0 & 0.93 & 20 & 0.16 \\
\hline 146 & NGC 3808A & 11:40:44.64 & $22: 26: 49.0$ & 107.2 & 9.90 & 2.17 & 5.52 & 4.0 & 0.60 & 5 & 0.17 \\
\hline 147 & NGC 3811 & 11:41:16.63 & $47: 41: 27.0$ & 54.2 & 9.19 & 2.30 & 3.82 & 14.5 & 0.71 & 6 & 0.14 \\
\hline
\end{tabular}


The Star Formation Reference Survey

TABLE $2-$ Continued

\begin{tabular}{|c|c|c|c|c|c|c|c|c|c|c|c|}
\hline SFRS & Name & $\begin{array}{r}\text { Posi } \\
\text { (J2 }\end{array}$ & $\begin{array}{l}\text { on }^{\mathrm{a}} \\
00)\end{array}$ & $\begin{array}{l}\text { Distance }^{\mathrm{b}} \\
(\mathrm{Mpc})\end{array}$ & $\begin{array}{c}\log L_{60} \\
\left(\mathrm{~L}_{\odot}\right)\end{array}$ & $F_{100} / F_{60}$ & $\begin{array}{c}K_{s}-F_{60} \\
(\mathrm{AB} \text { mag) }\end{array}$ & Weight & $\begin{array}{l}\text { Axial } \\
\text { Ratio }\end{array}$ & $\begin{array}{c}\mathrm{T} \\
\text { Type }\end{array}$ & $\begin{array}{c}\text { Extinction } \\
(B \text { mag })\end{array}$ \\
\hline 148 & NGC 3822 & $11: 42: 11.08$ & $10: 16: 40.0$ & 94.6 & 9.83 & 2.18 & 3.84 & 8.7 & 0.64 & -2 & 0.40 \\
\hline 149 & UGC 6665 & $11: 42: 12.22$ & $0: 20: 04.1$ & 85.0 & 9.81 & 1.16 & 5.84 & 8.1 & 0.53 & 3 & 0.17 \\
\hline 150 & MCG 3-30-051 & $11: 42: 24.50$ & 20:07:09.5 & 90.4 & 9.55 & 2.21 & 4.68 & 8.7 & 0.60 & $\ldots$ & 0.15 \\
\hline 151 & NGC 3839 & $11: 43: 54.34$ & $10: 47: 04.9$ & 91.3 & 9.91 & 1.84 & 5.29 & 13.2 & 0.65 & 8 & 0.40 \\
\hline 152 & UGC 6732 & $11: 45: 33.14$ & $58: 58: 41.2$ & 53.6 & 8.95 & 1.42 & 3.56 & 2.3 & 0.91 & $\ldots$ & 0.15 \\
\hline 153 & IC 730 & $11: 45: 35.26$ & $3: 13: 54.6$ & 93.1 & 9.82 & 1.81 & 4.66 & 13.2 & 0.65 & $\ldots$ & 0.14 \\
\hline 154 & IC 732 & $11: 45: 59.59$ & $20: 26: 49.8$ & 110.0 & 10.01 & 1.77 & 5.42 & 8.4 & 0.62 & 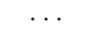 & 0.14 \\
\hline 155 & NGC 3912 & 11:50:04.46 & $26: 28: 45.3$ & $22.5 \pm 3.2$ & 8.95 & 1.93 & 4.34 & 9.5 & 0.47 & 3 & 0.16 \\
\hline 156 & NGC 3928 & $11: 51: 47.62$ & $48: 40: 59.3$ & $16.9 \pm 1.7$ & 8.42 & 2.04 & 3.81 & 4.2 & 0.90 & 3 & 0.13 \\
\hline 157 & NGC 3934 & $11: 52: 12.65$ & $16: 51: 06.7$ & 61.6 & 9.26 & 1.60 & 4.41 & 5.7 & 0.75 & $\ldots$ & 0.19 \\
\hline 158 & UGC 6865 & $11: 53: 39.96$ & $43: 27: 39.4$ & 91.2 & 9.67 & 2.14 & 4.20 & 7.4 & 0.31 & 17 & 0.12 \\
\hline 159 & UGC 6901 & $11: 55: 38.35$ & $43: 02: 45.1$ & 107.6 & 9.77 & 2.55 & 4.38 & 8.7 & 0.78 & 20 & 0.12 \\
\hline 160 & CGCG 013-010 & $11: 57: 05.93$ & 1:07:32.1 & 172.3 & 10.41 & 2.29 & 5.23 & 4.7 & 0.68 & $\cdots$ & 0.11 \\
\hline 161 & NGC 3991 & 11:57:30.96 & $32: 20: 13.3$ & 55.6 & 9.22 & 1.89 & 5.06 & 8.3 & 0.30 & 10 & 0.11 \\
\hline 162 & NGC 4004 & $11: 58: 05.23$ & $27: 52: 43.9$ & 57.9 & 9.41 & 1.85 & 5.27 & 8.3 & 0.46 & $\ldots$ & 0.13 \\
\hline 163 & NGC 4014 & $11: 58: 35.83$ & $16: 10: 38.1$ & 62.6 & 9.32 & 2.53 & 3.53 & 12.1 & 0.59 & 0 & 0.27 \\
\hline 164 & NGC 4010 & $11: 58: 37.90$ & $47: 15: 41.4$ & $17.1 \pm 0.8$ & 8.30 & 2.68 & 3.48 & 5.0 & 0.16 & 7 & 0.13 \\
\hline 165 & NGC 4018 & $11: 58: 40.78$ & $25: 18: 58.9$ & 72.6 & 9.44 & 2.92 & 4.49 & 5.5 & 0.33 & 2 & 0.12 \\
\hline 166 & NGC 4020 & $11: 58: 56.69$ & $30: 24: 42.8$ & $4.3 \pm 3.0$ & 7.26 & 2.57 & 7.02 & 1.0 & 0.47 & 7 & 0.11 \\
\hline 167 & IRAS 11571+3003 & $11: 59: 42.60$ & $29: 47: 12.5$ & 218.6 & 10.06 & 2.19 & 5.57 & 4.5 & 0.82 & & 0.10 \\
\hline 168 & UGC 7017 & $12: 02: 23.98$ & $14: 50: 37.1$ & 55.2 & 9.38 & 1.97 & 4.87 & 8.3 & 0.38 & 3 & 0.11 \\
\hline 169 & UGC 7016 & $12: 02: 22.51$ & $29: 51: 42.4$ & 110.3 & 9.64 & 2.37 & 3.51 & 3.8 & 0.34 & 2 & 0.25 \\
\hline 170 & MCG 3-31-030 & $12: 03: 35.95$ & $16: 03: 19.9$ & 13.1 & 7.11 & 2.75 & 3.83 & 1.0 & 0.62 & & 0.25 \\
\hline 171 & NGC 4062 & 12:04:03.84 & $31: 53: 44.9$ & $16.3 \pm 0.9$ & 7.73 & 3.79 & 2.22 & 13.2 & 0.38 & 5 & 0.12 \\
\hline 172 & NGC 4064 & 12:04:11.10 & $18: 26: 38.1$ & $8.5 \pm 1.53$ & 7.80 & 2.07 & 3.03 & 3.2 & 0.43 & 1 & 0.25 \\
\hline 173 & UGC 7104 & $12: 07: 09.55$ & $16: 59: 44.2$ & 102.3 & 9.93 & 2.14 & 5.09 & 8.7 & 0.82 & 3 & 0.23 \\
\hline 174 & NGC 4116 & $12: 07: 36.82$ & $2: 41: 32.3$ & $16.0 \pm 2.1$ & 8.18 & 2.94 & 4.32 & 2.3 & 0.51 & 8 & 0.15 \\
\hline 175 & NGC 4136 & $12: 09: 17.71$ & $29: 55: 39.4$ & $16.3 \pm 0.9$ & 7.40 & 2.56 & 3.69 & 1.7 & 0.98 & 5 & 0.15 \\
\hline 176 & NGC 4150 & $12: 10: 33.67$ & $30: 24: 05.8$ & $13.7 \pm 1.4$ & 6.26 & 1.98 & 2.23 & 1.0 & 0.66 & -2 & 0.11 \\
\hline 177 & IRAS $12086+1441$ & $12: 11: 14.38$ & $14: 24: 35.2$ & 13.1 & 8.04 & 1.41 & 6.18 & 5.3 & 0.70 & & 0.18 \\
\hline 178 & NGC 4162 & $12: 11: 53.04$ & $24: 07: 06.7$ & $42.5 \pm 5.0$ & 9.04 & 2.70 & 3.40 & 22.2 & 0.63 & 4 & 0.18 \\
\hline 179 & NGC 4178 & $12: 12: 46.92$ & $10: 52: 08.6$ & $16.8 \pm 0.8$ & 8.54 & 2.36 & 4.03 & 14.5 & 0.31 & 8 & 0.14 \\
\hline 180 & IRAS $12112+0305$ & $12: 13: 46.08$ & $2: 48: 41.5$ & 303.6 & 11.36 & 1.19 & 8.09 & 15.5 & 0.86 & & 0.12 \\
\hline 181 & NGC 4189 & $12: 13: 47.26$ & $13: 25: 29.3$ & $16.8 \pm 0.8$ & 8.46 & 2.64 & 3.51 & 5.0 & 0.81 & 6 & 0.18 \\
\hline 182 & NGC 4194 & $12: 14: 09.65$ & $54: 31: 35.9$ & 36.0 & 10.00 & 1.14 & 6.04 & 14.2 & 0.77 & 10 & 0.11 \\
\hline 183 & NGC 4204 & $12: 15: 14.45$ & $20: 39: 30.9$ & 10.0 & 7.26 & 2.02 & 5.15 & 1.0 & 0.40 & 8 & 0.21 \\
\hline 184 & NGC 4207 & $12: 15: 30.50$ & $9: 35: 05.6$ & $16.8 \pm 0.8$ & 8.42 & 2.46 & 3.67 & 5.0 & 0.47 & 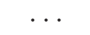 & 0.18 \\
\hline 185 & UGC 7286 & $12: 15: 59.26$ & $27: 26: 31.9$ & 115.4 & 9.47 & 1.79 & 6.79 & 3.0 & 0.41 & 2 & 0.19 \\
\hline 186 & NGC 4234 & $12: 17: 09.08$ & $3: 40: 50.2$ & 30.0 & 8.51 & 2.34 & 4.67 & 4.7 & 0.83 & 9 & 0.14 \\
\hline 187 & NGC 4237 & $12: 17: 11.42$ & $15: 19: 26.3$ & $16.8 \pm 0.8$ & 8.39 & 3.27 & 2.78 & 13.2 & 0.60 & 4 & 0.23 \\
\hline 188 & NGC 4244 & $12: 17: 29.45$ & $37: 48: 26.5$ & $4.3 \pm 0.21$ & 6.53 & 3.24 & 1.39 & 1.8 & 0.14 & 6 & 0.12 \\
\hline 189 & NGC 4253 & $12: 18: 26.52$ & $29: 48: 46.5$ & 64.9 & 9.57 & 1.05 & 4.39 & 4.8 & 0.83 & 1 & 0.20 \\
\hline 190 & MCG 3-32-005 & $12: 20: 47.23$ & $17: 00: 57.9$ & 13.1 & 8.38 & 1.92 & 5.24 & 2.8 & 0.72 & & 0.16 \\
\hline 191 & NGC 4290 & $12: 20: 47.52$ & 58:05:33.0 & 37.0 & 9.38 & 2.17 & 3.84 & 14.5 & 0.72 & 2 & 0.11 \\
\hline 192 & NGC 4294 & $12: 21: 17.81$ & $11: 30: 27.0$ & $16.8 \pm 0.8$ & 8.38 & 2.02 & 4.11 & 4.2 & 0.46 & 6 & 0.19 \\
\hline 193 & NGC 4314 & $12: 22: 32.02$ & $29: 53: 43.8$ & $16.3 \pm 0.9$ & 8.02 & 2.04 & 1.94 & 3.2 & 0.55 & 1 & 0.18 \\
\hline 194 & NGC 4385 & $12: 25: 42.79$ & $0: 34: 21.4$ & 29.0 & 9.17 & 1.32 & 4.59 & 6.9 & 0.56 & -1 & 0.13 \\
\hline 195 & NGC 4395 & $12: 25: 48.86$ & $33: 32: 48.7$ & $4.7 \pm 0.24$ & 6.79 & 3.53 & 4.60 & 1.8 & 0.73 & 9 & 0.11 \\
\hline 196 & NGC 4396 & $12: 25: 59.16$ & $15: 40: 15.6$ & $16.8 \pm 0.8$ & 8.04 & 3.02 & 4.07 & 2.3 & 0.31 & 7 & 0.21 \\
\hline 197 & NGC 4412 & $12: 26: 36.07$ & $3: 57: 52.9$ & $30.6 \pm 6.4$ & 9.02 & 1.97 & 4.01 & 9.5 & 0.91 & 3 & 0.15 \\
\hline 198 & NGC 4418 & $12: 26: 54.62$ & $-0: 52: 39.4$ & 29.0 & 10.06 & 0.77 & 7.27 & 14.2 & 0.50 & 1 & 0.14 \\
\hline 199 & NGC 4420 & $12: 26: 58.49$ & $2: 29: 39.7$ & $17.6 \pm 0.9$ & 8.55 & 2.62 & 3.78 & 12.1 & 0.50 & 4 & 0.15 \\
\hline 200 & NGC 4424 & $12: 27: 11.62$ & $9: 25: 14.4$ & 6.0 & 8.40 & 1.90 & 3.37 & 3.2 & 0.50 & 1 & 0.19 \\
\hline 201 & NGC 4435 & $12: 27: 40.46$ & $13: 04: 44.4$ & $16.7 \pm 1.7$ & 8.21 & 1.22 & 1.02 & 2.0 & 0.69 & -2 & 0.20 \\
\hline 202 & NGC 4438 & $12: 27: 45.62$ & 13:00:31.7 & $16.8 \pm 0.8$ & 8.53 & 2.52 & 1.80 & 22.2 & 0.43 & 20 & 0.20 \\
\hline 203 & NGC 4448 & $12: 28: 15.46$ & $28: 37: 13.1$ & $16.3 \pm 0.9$ & 7.33 & 3.11 & 1.33 & 2.0 & 0.36 & 2 & 0.16 \\
\hline 204 & $3 \mathrm{C} 273$ & $12: 29: 06.70$ & $2: 03: 08.6$ & 657.8 & 11.53 & 1.31 & 3.75 & 1.0 & 0.93 & & 0.12 \\
\hline 205 & NGC 4470 & $12: 29: 37.78$ & $7: 49: 27.1$ & $16.8 \pm 0.8$ & 8.21 & 2.43 & 3.83 & 4.6 & 0.64 & 1 & 0.18 \\
\hline 206 & IRAS $12274+0018$ & $12: 29: 58.85$ & $0: 01: 38.0$ & 38.1 & 8.48 & 1.33 & 5.33 & 3.0 & 0.90 & & 0.15 \\
\hline 207 & NGC 4491 & $12: 30: 57.12$ & 11:29:00.7 & $16.8 \pm 0.8$ & 8.36 & 1.25 & 4.06 & 2.3 & 0.53 & 1 & 0.19 \\
\hline 208 & NGC 4500 & $12: 31: 22.90$ & $29: 08: 11.3$ & 52.0 & 9.35 & 1.74 & 4.26 & 9.5 & 0.56 & 1 & 0.09 \\
\hline 209 & NGC 4495 & $12: 31: 22.15$ & $57: 57: 52.5$ & 74.2 & 9.59 & 2.22 & 4.43 & 8.7 & 0.53 & 2 & 0.14 \\
\hline 210 & IC 3476 & $12: 32: 41.88$ & 14:03:01.6 & $16.8 \pm 0.8$ & 8.05 & 2.42 & 4.60 & 1.0 & 0.62 & 10 & 0.24 \\
\hline 211 & NGC 4509 & $12: 33: 06.72$ & $32: 05: 34.5$ & 11.1 & 7.26 & 3.60 & 5.14 & 1.0 & 0.80 & 2 & 0.11 \\
\hline 212 & NGC 4519 & $12: 33: 30.26$ & $8: 39: 17.1$ & $16.8 \pm 0.8$ & 8.51 & 1.74 & 4.37 & 9.5 & 0.75 & 7 & 0.19 \\
\hline 213 & NGC 4548 & $12: 35: 26.45$ & $14: 29: 46.8$ & $16.2 \pm 1.6$ & 8.36 & 4.25 & 1.35 & 13.2 & 0.76 & 3 & 0.24 \\
\hline 214 & IRAS $12337+5044$ & $12: 36: 06.70$ & $50: 28: 18.7$ & 172.5 & 10.06 & 2.17 & 5.55 & 4.5 & 0.53 & & 0.13 \\
\hline 215 & IC 3581 & $12: 36: 38.06$ & $24: 25: 43.6$ & 106.1 & 10.02 & 1.27 & 5.23 & 6.0 & 0.71 & $\cdots$ & 0.09 \\
\hline 216 & NGC 4592 & $12: 39: 18.74$ & $-0: 31: 55.0$ & $11.1 \pm 1.2$ & 8.29 & 2.18 & 4.00 & 4.6 & 0.30 & 8 & 0.22 \\
\hline 217 & NGC 4607 & $12: 41: 12.22$ & $11: 53: 11.9$ & $16.8 \pm 0.8$ & 8.38 & 2.85 & 3.62 & 13.2 & 0.25 & 3 & 0.13 \\
\hline 218 & NGC 4625 & $12: 41: 52.73$ & $41: 16: 26.3$ & $9.2 \pm 0.57$ & 7.37 & 3.12 & 3.17 & 1.8 & 0.79 & 9 & 0.15 \\
\hline 219 & NGC 4630 & $12: 42: 31.13$ & $3: 57: 36.9$ & $15.6 \pm 1.0$ & 7.42 & 2.30 & 4.09 & 2.0 & 0.72 & 10 & 0.22 \\
\hline 220 & IC 3690 & $12: 42: 49.20$ & $10: 21: 26.9$ & 13.1 & 8.10 & 1.51 & 4.71 & 3.0 & 0.59 & 20 & 0.17 \\
\hline 221 & UGC 7905 & $12: 43: 47.93$ & $54: 53: 45.2$ & 78.7 & 9.43 & 1.39 & 7.46 & 4.3 & 0.58 & 17 & 0.12 \\
\hline
\end{tabular}


Ashby et al.

TABLE $2-$ Continued

\begin{tabular}{|c|c|c|c|c|c|c|c|c|c|c|c|}
\hline SFRS & Name & $\begin{array}{r}\text { Posi } \\
\text { (J2 }\end{array}$ & $\begin{array}{l}\text { on }^{\mathrm{a}} \\
00)\end{array}$ & $\begin{array}{l}\text { Distance }^{\mathrm{b}} \\
(\mathrm{Mpc})\end{array}$ & $\begin{array}{c}\log L_{60} \\
\left(\mathrm{~L}_{\odot}\right)\end{array}$ & $F_{100} / F_{60}$ & $\begin{array}{c}K_{s}-F_{60} \\
(\mathrm{AB} \text { mag) }\end{array}$ & Weight & $\begin{array}{l}\text { Axial } \\
\text { Ratio }\end{array}$ & $\begin{array}{c}\mathrm{T} \\
\text { Type }\end{array}$ & $\begin{array}{c}\text { Extinction } \\
(B \text { mag })\end{array}$ \\
\hline 222 & MCG 5-30-069 & $12: 44: 41.26$ & $26: 25: 10.5$ & 74.0 & 9.57 & 1.66 & 5.28 & 9.8 & 0.73 & $\ldots$ & 0.12 \\
\hline 223 & IC 3721 & $12: 44: 53.11$ & $18: 45: 18.9$ & 98.5 & 9.67 & 2.05 & 4.11 & 7.4 & 0.45 & 20 & 0.15 \\
\hline 224 & NGC 4670 & $12: 45: 17.26$ & $27: 07: 32.2$ & $14.3 \pm 1.1$ & 8.29 & 1.64 & 4.53 & 2.3 & 0.55 & 15 & 0.12 \\
\hline 225 & NGC 4675 & $12: 45: 31.90$ & $54: 44: 15.4$ & 76.9 & 9.54 & 1.99 & 4.66 & 13.2 & 0.38 & 3 & 0.12 \\
\hline 226 & MCG 7-26-051 & $12: 46: 56.83$ & $42: 15: 59.1$ & 146.9 & 10.22 & 2.16 & 5.17 & 4.7 & 0.60 & & 0.13 \\
\hline 227 & NGC 4689 & $12: 47: 46.51$ & $4: 20: 09.8$ & $16.8 \pm 0.8$ & 8.37 & 3.72 & 2.51 & 13.2 & 0.81 & 4 & 0.19 \\
\hline 228 & NGC 4688 & $12: 47: 45.55$ & $13: 45: 46.1$ & $15.6 \pm 1.0$ & 7.95 & 2.05 & 4.61 & 2.8 & 0.74 & 6 & 0.23 \\
\hline 229 & NGC 4704 & $12: 48: 46.44$ & $41: 55: 16.5$ & 122.8 & 9.81 & 1.36 & 4.47 & 4.8 & 0.87 & 4 & 0.14 \\
\hline 230 & NGC 4701 & $12: 49: 11.59$ & $3: 23: 19.4$ & $15.6 \pm 1.0$ & 7.44 & 2.44 & 3.97 & 2.0 & 0.82 & 6 & 0.27 \\
\hline 231 & IRAS $12468+3436$ & $12: 49: 17.16$ & $34: 19: 43.0$ & 498.3 & 10.87 & 2.41 & 7.19 & 3.5 & 0.78 & & 0.10 \\
\hline 232 & IRAS $12470+1404$ & $12: 49: 34.80$ & $13: 48: 09.8$ & 13.1 & 7.81 & 1.84 & 6.14 & 2.0 & 0.70 & & 0.17 \\
\hline 233 & MCG 8-23-097 & $12: 50: 39.84$ & $47: 56: 00.3$ & 131.2 & 10.36 & 1.59 & 8.02 & 14.2 & 0.65 & . & 0.11 \\
\hline 234 & NGC 4747 & $12: 51: 45.60$ & $25: 46: 30.1$ & $14.3 \pm 1.1$ & 8.20 & 1.99 & 4.06 & 4.2 & 0.72 & 6 & 0.08 \\
\hline 235 & UGC 8017 & $12: 52: 53.59$ & $28: 22: 16.6$ & 107.1 & 9.72 & 2.75 & 3.94 & 5.0 & 0.41 & & 0.07 \\
\hline 236 & NGC 4765 & $12: 53: 14.57$ & $4: 27: 47.7$ & $15.6 \pm 1.0$ & 7.39 & 1.87 & 4.75 & 1.0 & 0.67 & . & 0.27 \\
\hline 237 & VCC 2096 & $12: 53: 24.79$ & $11: 42: 36.4$ & 13.1 & 8.09 & 1.63 & 5.94 & 5.3 & 0.68 & .. & 0.16 \\
\hline 238 & UGC 8041 & $12: 55: 12.65$ & 0:06:59.9 & $23.0 \pm 1.6$ & 7.86 & 2.47 & 4.99 & 1.0 & 0.48 & 7 & 0.18 \\
\hline 239 & UGC 8058 & $12: 56: 14.23$ & $56: 52: 25.3$ & 179.6 & 11.48 & 0.92 & 5.62 & 15.5 & 0.95 & 5 & 0.11 \\
\hline 240 & NGC 4837 & $12: 56: 48.31$ & $48: 17: 48.9$ & 132.5 & 9.94 & 2.19 & 4.21 & 8.7 & 0.46 & & 0.13 \\
\hline 241 & UM 530 & $12: 58: 08.35$ & 1:51:44.4 & 282.7 & 10.15 & 2.92 & 5.75 & 1.5 & 0.64 & 0 & 0.18 \\
\hline 242 & NGC 4861 & 12:59:01.92 & $34: 51: 21.4$ & $18.5 \pm 1.1$ & 7.78 & 1.20 & 6.88 & 5.3 & 0.53 & 9 & 0.09 \\
\hline 243 & NGC 4868 & $12: 59: 08.90$ & $37: 18: 37.4$ & 74.0 & 9.62 & 2.56 & 3.90 & 8.7 & 0.95 & 2 & 0.10 \\
\hline 244 & NGC 4922 & $13: 01: 25.27$ & $29: 18: 49.5$ & 107.2 & 10.27 & 1.07 & 4.96 & 6.0 & 0.87 & 90 & 0.07 \\
\hline 245 & UGC 8179 & $13: 05: 14.16$ & $31: 59: 59.0$ & 222.1 & 10.10 & 2.37 & 3.74 & 1.0 & 0.46 & 3 & 0.07 \\
\hline 246 & NGC 5001 & 13:09:33.12 & $53: 29: 39.4$ & 134.8 & 10.11 & 2.32 & 4.53 & 2.7 & 0.56 & 20 & 0.14 \\
\hline 247 & IC 856 & $13: 10: 41.33$ & $20: 32: 10.6$ & 64.3 & 9.15 & 2.20 & 4.56 & 4.7 & 0.44 & . & 0.15 \\
\hline 248 & UGC 8269 & $13: 11: 15.12$ & $46: 42: 02.3$ & 124.1 & 10.06 & 2.06 & 5.63 & 8.4 & 0.44 & 20 & 0.10 \\
\hline 249 & NGC 5014 & $13: 11: 37.03$ & $22: 54: 55.8$ & $18.5 \pm 1.1$ & 8.30 & 1.85 & 4.02 & 4.2 & 0.38 & 1 & 0.05 \\
\hline 250 & NGC 5012 & $13: 11: 31.22$ & $36: 16: 55.7$ & $40.2 \pm 6.2$ & 9.02 & 2.79 & 2.94 & 22.2 & 0.54 & 5 & 0.08 \\
\hline 251 & IRAS $13116+4508$ & $13: 13: 47.88$ & $44: 52: 58.6$ & 258.3 & 10.24 & 2.19 & 5.55 & 4.5 & 0.61 & & 0.10 \\
\hline 252 & IC 860 & $13: 15: 03.50$ & $24: 37: 07.8$ & 54.5 & 10.02 & 1.06 & 6.74 & 14.2 & 0.66 & . & 0.06 \\
\hline 253 & IRAS $13144+4508$ & $13: 16: 39.74$ & $44: 52: 35.0$ & 381.8 & 10.52 & 2.04 & 4.30 & 1.0 & 0.89 & $\cdots$ & 0.09 \\
\hline 254 & NGC 5060 & $13: 17: 16.22$ & $6: 02: 14.8$ & 97.4 & 9.83 & 2.25 & 4.67 & 8.7 & 0.68 & 3 & 0.31 \\
\hline 255 & UGC 8357 & $13: 17: 58.80$ & $-0: 18: 42.0$ & 146.9 & 10.14 & 1.83 & 5.06 & 7.4 & 0.49 & 17 & 0.32 \\
\hline 256 & UGC 8361 & $13: 18: 18.58$ & $6: 20: 07.4$ & 106.3 & 9.87 & 1.60 & 4.87 & 9.8 & 0.49 & 2 & 0.28 \\
\hline 257 & IC 883 & $13: 20: 35.40$ & $34: 08: 21.6$ & 104.7 & 10.60 & 1.61 & 6.87 & 15.5 & 0.84 & 10 & 0.06 \\
\hline 258 & NGC 5100 & $13: 20: 59.59$ & $8: 58: 41.9$ & 142.2 & 10.04 & 2.21 & 4.27 & 2.7 & 0.65 & 17 & 0.28 \\
\hline 259 & NGC 5104 & 13:21:23.09 & $0: 20: 32.7$ & 87.8 & 10.08 & 1.82 & 4.87 & 7.4 & 0.56 & 1 & 0.32 \\
\hline 260 & NGC 5107 & $13: 21: 24.70$ & $38: 32: 15.4$ & $18.5 \pm 1.1$ & 7.76 & 1.88 & 4.79 & 2.8 & 0.34 & 7 & 0.07 \\
\hline 261 & NGC 5112 & $13: 21: 56.40$ & $38: 44: 05.0$ & $18.5 \pm 1.1$ & 8.22 & 2.46 & 4.38 & 4.6 & 0.62 & 6 & 0.07 \\
\hline 262 & NGC 5123 & $13: 23: 10.51$ & $43: 05: 10.5$ & 123.4 & 9.81 & 3.14 & 3.69 & 4.8 & 0.94 & 6 & 0.12 \\
\hline 263 & IRAS $13218+0552$ & $13: 24: 19.90$ & $5: 37: 04.7$ & 850.4 & 11.54 & 0.80 & 5.28 & 1.0 & 0.96 & & 0.25 \\
\hline 264 & IRAS $13232+1731$ & $13: 25: 43.87$ & $17: 15: 52.8$ & 331.8 & 10.34 & 3.06 & 4.49 & 1.0 & 0.79 & . & 0.18 \\
\hline 265 & NGC 5147 & $13: 26: 19.73$ & $2: 06: 03.1$ & 18.0 & 8.40 & 2.34 & 4.10 & 4.6 & 0.75 & 8 & 0.22 \\
\hline 266 & NGC 5204 & $13: 29: 36.58$ & $58: 25: 13.3$ & $3.2 \pm 0.16$ & 7.29 & 1.76 & 4.20 & 1.7 & 0.60 & 9 & 0.12 \\
\hline 267 & UGC 8502 & $13: 30: 39.36$ & $31: 17: 02.6$ & 149.9 & 9.97 & 1.74 & 6.26 & 7.0 & 0.53 & 17 & 0.09 \\
\hline 268 & UGC 8561 & $13: 34: 57.26$ & $34: 02: 38.7$ & 107.5 & 9.89 & 2.49 & 4.84 & 8.7 & 0.84 & 6 & 0.07 \\
\hline 259 & NGC 5230 & $13: 35: 31.87$ & $13: 40: 34.2$ & 105.6 & 9.62 & 3.17 & 3.57 & 4.8 & 0.93 & 5 & 0.17 \\
\hline 270 & IRAS $13349+2438$ & $13: 37: 18.72$ & $24: 23: 03.4$ & 453.5 & 10.66 & 1.45 & 2.99 & 1.0 & 0.83 & & 0.09 \\
\hline 271 & NGC 5256 & $13: 38: 23.47$ & $6: 53: 15.6$ & 125.2 & 10.47 & 1.68 & 5.27 & 6.0 & 0.58 & . & 0.10 \\
\hline 272 & UGC 8626 & $13: 38: 17.11$ & $48: 16: 36.1$ & 108.8 & 9.60 & 1.55 & 4.35 & 4.8 & 0.45 & 4 & 0.24 \\
\hline 273 & NGC 5263 & $13: 39: 55.66$ & $28: 24: 02.7$ & 77.5 & 9.69 & 2.03 & 4.58 & 13.2 & 0.33 & 20 & 0.10 \\
\hline 274 & MCG 1-35-028 & $13: 40: 27.19$ & $4: 46: 25.8$ & 105.1 & 9.85 & 1.54 & 4.89 & 9.8 & 0.74 & $\ldots$ & 0.24 \\
\hline 275 & IC 910 & $13: 41: 07.85$ & $23: 16: 55.4$ & 120.3 & 10.26 & 1.24 & 7.62 & 14.2 & 0.89 & $\ldots$ & 0.13 \\
\hline 276 & MK 268 & 13:41:11.14 & $30: 22: 41.3$ & 173.7 & 10.06 & 1.25 & 4.35 & 1.7 & 0.65 & 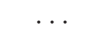 & 0.12 \\
\hline 277 & NGC 5278 & $13: 41: 39.24$ & $55: 40: 14.1$ & 114.4 & 9.70 & 2.91 & 3.74 & 4.8 & 0.71 & 3 & 0.12 \\
\hline 278 & NGC 5273 & $13: 42: 08.38$ & $35: 39: 15.5$ & $16.5 \pm 1.6$ & 7.83 & 1.40 & 1.55 & 2.0 & 0.91 & -2 & 0.10 \\
\hline 279 & UGC 8685 & $13: 43: 08.83$ & $30: 20: 15.9$ & 152.6 & 10.14 & 2.25 & 4.85 & 4.7 & 0.78 & 4 & 0.11 \\
\hline 280 & UGC 8686 & $13: 43: 40.13$ & $3: 53: 47.3$ & 105.4 & 9.69 & 2.37 & 4.47 & 8.7 & 0.33 & 20 & 0.24 \\
\hline 281 & UGC 8696 & $13: 44: 42.17$ & $55: 53: 13.6$ & 163.0 & 11.23 & 0.94 & 7.32 & 15.5 & 0.45 & . & 0.12 \\
\hline 282 & NGC 5297 & $13: 46: 49.46$ & $14: 24: 01.7$ & $30.9 \pm 1.9$ & 8.92 & 3.05 & 2.93 & 22.2 & 0.32 & 5 & 0.08 \\
\hline 283 & MK 796 & $13: 46: 23.66$ & $43: 52: 20.4$ & 98.5 & 9.85 & 1.72 & 5.34 & 7.0 & 0.88 & & 0.15 \\
\hline 284 & IRAS 13446+1121 & $13: 47: 04.37$ & $11: 06: 22.6$ & 104.5 & 9.95 & 1.05 & 5.57 & 8.1 & 0.81 & $\cdots$ & 0.16 \\
\hline 285 & NGC 5303 & $13: 47: 45.00$ & $38: 18: 16.7$ & 23.0 & 8.61 & 2.10 & 4.46 & 9.5 & 0.57 & 15 & 0.12 \\
\hline 286 & NGC 5313 & $13: 49: 44.35$ & $39: 59: 05.2$ & $30.9 \pm 1.9$ & 9.07 & 3.41 & 3.06 & 22.2 & 0.53 & 3 & 0.08 \\
\hline 287 & MCG 3-35-034 & 13:53:09.67 & $14: 39: 20.9$ & 178.6 & 10.01 & 2.33 & 5.78 & 4.5 & 0.73 & 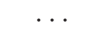 & 0.17 \\
\hline 288 & NGC 5347 & $13: 53: 17.78$ & $33: 29: 27.1$ & 39.0 & 8.69 & 1.88 & 3.16 & 4.3 & 0.78 & 2 & 0.12 \\
\hline 289 & NGC 5350 & $13: 53: 21.62$ & $40: 21: 50.2$ & $30.9 \pm 1.9$ & 8.94 & 2.98 & 2.78 & 22.2 & 0.75 & 3 & 0.10 \\
\hline 290 & NGC 5368 & 13:54:31.18 & $15: 02: 38.9$ & 74.6 & 9.23 & 2.08 & 3.56 & 4.3 & 0.76 & 2 & 0.12 \\
\hline 291 & UGC 8827 & $13: 54: 29.16$ & $54: 19: 50.4$ & 85.4 & 9.80 & 1.49 & 4.82 & 9.8 & 0.96 & -2 & 0.12 \\
\hline 292 & UGC 8850 & $13: 56: 02.62$ & $18: 22: 17.8$ & 216.5 & 10.45 & 0.86 & 3.90 & 1.7 & 0.88 & 15 & 0.12 \\
\hline 293 & UGC 8856 & $13: 56: 07.90$ & $30: 04: 52.9$ & 137.9 & 9.99 & 2.15 & 5.44 & 4.0 & 0.48 & 17 & 0.11 \\
\hline 294 & NGC 5374 & $13: 57: 29.64$ & $6: 05: 49.2$ & 68.9 & 9.45 & 2.49 & 3.94 & 14.5 & 0.77 & 4 & 0.23 \\
\hline 295 & UGC 8902 & $13: 59: 02.81$ & $15: 33: 56.7$ & 114.4 & 9.60 & 2.59 & 3.24 & 3.8 & 0.39 & 3 & 0.11 \\
\hline
\end{tabular}


The Star Formation Reference Survey

TABLE $2-$ Continued

\begin{tabular}{|c|c|c|c|c|c|c|c|c|c|c|c|}
\hline \multirow{2}{*}{$\begin{array}{l}\text { SFRS } \\
296\end{array}$} & \multirow{2}{*}{$\begin{array}{l}\text { Name } \\
\text { NGC } 5403\end{array}$} & \multicolumn{2}{|c|}{$\begin{array}{l}\text { Position }^{\mathrm{a}} \\
(\mathrm{J} 2000)\end{array}$} & \multirow{2}{*}{$\begin{array}{r}\begin{array}{c}\text { Distance } \\
(\mathrm{Mpc})\end{array} \\
37.0\end{array}$} & \multirow{2}{*}{$\begin{array}{r}\begin{array}{c}\log L_{60} \\
\left(\mathrm{~L}_{\odot}\right)\end{array} \\
9.11\end{array}$} & \multirow{2}{*}{$\begin{array}{c}F_{100} / F_{60} \\
3.68\end{array}$} & $\begin{array}{c}K_{s}-F_{60} \\
(\mathrm{AB} \text { mag) }\end{array}$ & Weight & $\begin{array}{l}\text { Axial } \\
\text { Ratio }\end{array}$ & $\begin{array}{c}\mathrm{T} \\
\text { Type }\end{array}$ & $\begin{array}{c}\text { Extinction } \\
(B \text { mag })\end{array}$ \\
\hline & & $13: 59: 50.76$ & $38: 10: 56.2$ & & & & 4.68 & 1.5 & 0.32 & 3 & 0.10 \\
\hline 297 & MCG 7-29-036 & 14:00:57.84 & $42: 51: 20.4$ & 144.6 & 10.13 & 1.72 & 6.10 & 8.4 & 0.45 & $\ldots$ & 0.07 \\
\hline 298 & NGC 5414 & $14: 02: 03.53$ & $9: 55: 45.6$ & 68.3 & 9.40 & 1.66 & 5.23 & 6.9 & 0.67 & 15 & 0.14 \\
\hline 299 & MCG 5-33-046 & 14:04:48.00 & $30: 44: 37.3$ & 116.4 & 9.96 & 1.54 & 5.16 & 9.8 & 0.59 & 20 & 0.11 \\
\hline 300 & NGC 5474 & 14:05:01.42 & $53: 39: 44.4$ & $5.6 \pm 0.43$ & 7.32 & 2.45 & 3.70 & 1.7 & 0.58 & 6 & 0.13 \\
\hline 301 & NGC 5480 & $14: 06: 21.58$ & $50: 43: 30.4$ & $30.5 \pm 3.6$ & 9.00 & 2.77 & 4.05 & 5.5 & 0.79 & 5 & 0.12 \\
\hline 302 & MCG 6-31-070 & $14: 06: 49.08$ & $33: 46: 18.3$ & 155.8 & 10.22 & 1.87 & 5.35 & 8.4 & 0.67 & & 0.13 \\
\hline 303 & CGCG 74-129 & $14: 10: 41.35$ & $13: 33: 28.8$ & 76.5 & 9.67 & 0.81 & 5.86 & 8.1 & 0.74 & $\cdots$ & 0.12 \\
\hline 304 & NGC 5520 & $14: 12: 38.16$ & $39: 18: 36.6$ & $30.5 \pm 3.6$ & 8.62 & 2.49 & 3.94 & 14.5 & 0.55 & 3 & 0.11 \\
\hline 305 & NGC 5515 & $14: 12: 22.80$ & $50: 20: 54.4$ & 114.1 & 9.76 & 2.67 & 3.58 & 3.8 & 0.60 & 2 & 0.10 \\
\hline 306 & NGC 5526 & $14: 13: 53.76$ & $57: 46: 16.8$ & $27.9 \pm 2.3$ & 8.84 & 2.33 & 6.33 & 2.3 & 0.23 & 4 & 0.11 \\
\hline 307 & NGC 5522 & $14: 14: 50.38$ & $15: 08: 48.8$ & 72.1 & 9.36 & 2.08 & 3.71 & 4.3 & 0.31 & 3 & 0.12 \\
\hline 308 & NGC 5541 & $14: 16: 31.80$ & $39: 35: 20.6$ & 115.4 & 9.90 & 2.23 & 3.96 & 8.7 & 0.74 & 20 & 0.10 \\
\hline 309 & IC 4395 & $14: 17: 21.07$ & $26: 51: 26.8$ & 160.4 & 10.29 & 1.95 & 7.12 & 8.4 & 0.90 & 20 & 0.11 \\
\hline 310 & UGC 9165 & $14: 18: 47.78$ & $24: 56: 25.9$ & 81.3 & 9.66 & 2.32 & 4.51 & 8.7 & 0.37 & 20 & 0.15 \\
\hline 311 & MK 1490 & $14: 19: 43.22$ & $49: 14: 11.9$ & 116.2 & 10.33 & 1.35 & 6.27 & 14.2 & 0.92 & .. & 0.13 \\
\hline 312 & NGC 5585 & $14: 19: 48.19$ & $56: 43: 45.6$ & $5.6 \pm 0.43$ & 7.33 & 2.59 & 3.63 & 1.7 & 0.57 & 7 & 0.12 \\
\hline 313 & IC 4408 & $14: 21: 13.10$ & $29: 59: 36.6$ & 134.9 & 9.93 & 2.55 & 4.25 & 8.7 & 0.48 & 20 & 0.10 \\
\hline 314 & NGC 5584 & $14: 22: 23.76$ & $-0: 23: 15.6$ & $23.1 \pm 4.8$ & 8.67 & 2.39 & 3.93 & 14.5 & 0.70 & 6 & 0.33 \\
\hline 315 & NGC 5633 & $14: 27: 28.39$ & $46: 08: 47.5$ & $36.5 \pm 7.6$ & 9.02 & 2.94 & 3.67 & 22.2 & 0.67 & 3 & 0.16 \\
\hline 316 & NGC 5660 & $14: 29: 49.82$ & $49: 37: 21.6$ & $38.9 \pm 3.0$ & 9.21 & 2.21 & 4.22 & 14.5 & 0.92 & 5 & 0.14 \\
\hline 317 & NGC 5656 & $14: 30: 25.51$ & $35: 19: 15.7$ & 53.7 & 9.13 & 1.73 & 3.95 & 22.2 & 0.83 & 3 & 0.10 \\
\hline 318 & NGC 5657 & $14: 30: 43.15$ & $29: 10: 49.9$ & 64.4 & 9.19 & 2.93 & 3.45 & 9.5 & 0.47 & 2 & 0.08 \\
\hline 319 & CGCG 133-083 & $14: 31: 54.10$ & $21: 56: 18.3$ & 190.6 & 10.34 & 1.79 & 5.94 & 8.4 & 0.85 & & 0.24 \\
\hline 320 & MCG 7-30-028 & $14: 33: 48.36$ & $40: 05: 38.9$ & 116.1 & 9.79 & 2.68 & 4.76 & 8.7 & 0.95 & 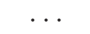 & 0.10 \\
\hline 321 & MCG 6-32-070 & $14: 35: 18.38$ & $35: 07: 07.2$ & 127.0 & 10.07 & 2.03 & 4.88 & 7.4 & 0.94 & $\cdots$ & 0.07 \\
\hline 322 & UGC 9412 & $14: 36: 22.08$ & $58: 47: 39.3$ & 138.7 & 10.06 & 1.02 & 4.22 & 1.7 & 0.80 & 11 & 0.11 \\
\hline 323 & NGC 5698 & $14: 37: 14.69$ & $38: 27: 15.4$ & 60.9 & 8.99 & 2.06 & 3.73 & 4.3 & 0.53 & 3 & 0.08 \\
\hline 324 & NGC 5691 & $14: 37: 53.33$ & $-0: 23: 55.9$ & $19.8 \pm 1.0$ & 8.90 & 1.92 & 4.37 & 9.5 & 0.69 & 1 & 0.33 \\
\hline 325 & MCG 9-24-035 & $14: 45: 45.12$ & $51: 34: 50.9$ & 137.4 & 10.19 & 1.30 & 4.82 & 6.0 & 0.70 & $\ldots$ & 0.13 \\
\hline 326 & MCG 9-24-038 & $14: 46: 37.08$ & $56: 13: 58.7$ & 166.6 & 10.01 & 2.40 & 4.73 & 4.7 & 0.60 & $\cdots$ & 0.11 \\
\hline 327 & UGC 9560 & $14: 50: 56.57$ & $35: 34: 19.6$ & 23.0 & 7.85 & 2.06 & 5.55 & 2.0 & 0.54 & $\ldots$ & 0.10 \\
\hline 328 & IC 1076 & $14: 54: 59.62$ & 18:02:14.4 & 92.5 & 9.51 & 2.14 & 4.57 & 8.7 & 0.60 & $\ldots$ & 0.20 \\
\hline 329 & IRAS $14538+1730$ & $14: 56: 08.54$ & $17: 18: 34.4$ & 432.9 & 10.92 & 2.04 & 5.82 & 6.6 & 0.61 & $\ldots$ & 0.17 \\
\hline 330 & NGC 5795 & $14: 56: 19.34$ & $49: 23: 55.4$ & $38.2 \pm 7.9$ & 9.02 & 2.64 & 3.94 & 14.5 & 0.26 & 20 & 0.16 \\
\hline 331 & UGC 9618 & $14: 57: 00.79$ & $24: 37: 02.2$ & 145.8 & 10.55 & 2.41 & 5.28 & 1.5 & 0.54 & 17 & 0.21 \\
\hline 332 & UGC 9639 & $14: 58: 36.00$ & $44: 53: 01.0$ & 157.7 & 10.21 & 2.12 & 4.52 & 1.5 & 0.82 & 2 & 0.14 \\
\hline 333 & MCG 6-33-022 & $15: 08: 05.98$ & $34: 23: 27.2$ & 194.5 & 10.43 & 2.03 & 5.76 & 8.4 & 0.58 & 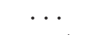 & 0.13 \\
\hline 334 & NGC 5879 & 15:09:46.78 & $57: 00: 00.8$ & $15.5 \pm 0.9$ & 8.37 & 2.76 & 3.11 & 13.2 & 0.38 & 4 & 0.12 \\
\hline 335 & MCG 9-25-036 & $15: 12: 52.33$ & $51: 23: 55.0$ & 160.1 & 9.65 & 2.94 & 5.68 & 2.0 & 0.64 & $\ldots$ & 0.16 \\
\hline 336 & NGC 5899 & $15: 15: 03.22$ & $42: 02: 59.5$ & $43.5 \pm 4.4$ & 9.09 & 3.60 & 2.87 & 22.2 & 0.34 & 5 & 0.16 \\
\hline 337 & NGC 5905 & $15: 15: 23.33$ & $55: 31: 02.3$ & 58.7 & 9.32 & 2.39 & 3.71 & 12.1 & 0.79 & 3 & 0.11 \\
\hline 338 & MK 848 & $15: 18: 06.14$ & $42: 44: 45.1$ & 173.9 & 10.89 & 1.09 & 7.02 & 15.5 & 0.61 & -2 & 0.16 \\
\hline 339 & IC 4553 & $15: 34: 57.22$ & $23: 30: 13.2$ & 83.5 & 11.21 & 1.13 & 7.94 & 15.5 & 0.86 & 15 & 0.26 \\
\hline 340 & UGC 9922 & $15: 35: 53.88$ & $38: 40: 31.8$ & 86.7 & 9.61 & 1.86 & 5.26 & 13.2 & 0.53 & 17 & 0.13 \\
\hline 341 & IC 4567 & $15: 37: 13.27$ & $43: 17: 53.9$ & 88.6 & 9.61 & 2.89 & 3.89 & 5.0 & 0.71 & 6 & 0.17 \\
\hline 342 & MCG 4-37-016 & $15: 39: 27.50$ & $24: 56: 51.4$ & 102.9 & 9.76 & 1.27 & 6.60 & 8.1 & 0.74 & $\ldots$ & 0.28 \\
\hline 343 & NGC 5975 & $15: 39: 57.96$ & $21: 28: 14.3$ & 69.3 & 9.60 & 1.87 & 4.41 & 7.4 & 0.51 & 20 & 0.29 \\
\hline 344 & NGC 5980 & $15: 41: 30.43$ & $15: 47: 15.6$ & 65.2 & 9.47 & 2.58 & 3.75 & 12.1 & 0.41 & 20 & 0.22 \\
\hline 345 & NGC 5992 & $15: 44: 21.50$ & 41:05:10.9 & 140.2 & 9.89 & 2.85 & 5.00 & 3.0 & 0.90 & 20 & 0.16 \\
\hline 346 & NGC 5996 & $15: 46: 58.87$ & $17: 53: 03.0$ & 54.0 & 9.37 & 1.85 & 5.15 & 8.3 & 0.80 & $\cdots$ & 0.21 \\
\hline 347 & IRAS 15519+3537 & $15: 53: 48.86$ & $35: 28: 02.2$ & 354.1 & 10.53 & 2.26 & 5.72 & 3.5 & 0.76 & $\ldots$ & 0.17 \\
\hline 348 & UGC 10099 & $15: 56: 36.41$ & $41: 52: 50.5$ & 152.2 & 10.06 & 1.85 & 5.31 & 7.4 & 0.97 & 11 & 0.13 \\
\hline 349 & MCG 5-38-006 & $15: 58: 43.70$ & $26: 49: 05.3$ & 69.6 & 9.51 & 1.00 & 4.94 & 9.8 & 0.87 & $\ldots$ & 0.27 \\
\hline 350 & UGC 10120 & $15: 59: 11.18$ & $20: 45: 16.8$ & 138.9 & 9.50 & 2.09 & 3.63 & 1.7 & 0.82 & 3 & 0.17 \\
\hline 351 & NGC 6027A & 15:59:09.62 & $35: 01: 47.5$ & 70.6 & 9.04 & 2.14 & 3.65 & 4.3 & 0.69 & 1 & 0.27 \\
\hline 352 & NGC 6040A & $16: 04: 26.52$ & $17: 44: 31.2$ & 177.0 & 9.81 & 1.74 & 3.69 & 1.7 & 0.54 & 5 & 0.29 \\
\hline 353 & UGC 10200 & $16: 05: 45.89$ & $41: 20: 41.1$ & $31.2 \pm 6.5$ & 8.55 & 1.27 & 5.03 & 6.9 & 0.88 & $\cdots$ & 0.12 \\
\hline 354 & IRAS $16052+5334$ & $16: 06: 33.00$ & $53: 26: 32.1$ & 366.1 & 10.52 & 2.96 & 5.38 & 1.5 & 0.76 & $\ldots$ & 0.11 \\
\hline 355 & IRAS $16053+1836$ & $16: 07: 38.52$ & $18: 28: 48.3$ & 161.4 & 10.16 & 1.57 & 5.12 & 6.0 & 0.89 & $\ldots$ & 0.26 \\
\hline 356 & NGC 6090 & $16: 11: 40.32$ & $52: 27: 23.1$ & 131.2 & 10.47 & 1.54 & 6.16 & 14.2 & 0.69 & 17 & 0.17 \\
\hline 357 & UGC 10273 & $16: 12: 44.69$ & $28: 17: 10.0$ & 111.3 & 9.73 & 2.46 & 5.76 & 4.0 & 0.23 & 15 & 0.26 \\
\hline 358 & IRAS $16150+2233$ & $16: 17: 08.95$ & $22: 26: 28.0$ & 278.1 & 10.14 & 7.02 & 5.38 & 1.5 & 0.62 & 0 & 0.37 \\
\hline 359 & UGC 10322 & $16: 18: 07.85$ & $22: 13: 32.3$ & 69.1 & 9.30 & 2.85 & 4.29 & 5.5 & 0.36 & 20 & 0.37 \\
\hline 360 & NGC 6120 & $16: 19: 48.12$ & $37: 46: 27.7$ & 134.9 & 10.28 & 2.06 & 4.98 & 7.4 & 0.83 & $\cdots$ & 0.12 \\
\hline 361 & MCG 3-42-004 & $16: 24: 15.17$ & $20: 11: 00.8$ & 171.9 & 10.02 & 2.19 & 4.63 & 4.7 & 0.87 & $\ldots$ & 0.34 \\
\hline 362 & UGC 10407 & $16: 28: 27.89$ & $41: 13: 03.5$ & 124.7 & 9.84 & 1.80 & 5.65 & 7.0 & 0.79 & $\ldots$ & 0.10 \\
\hline 363 & IRAS $16320+3922$ & $16: 33: 49.63$ & $39: 15: 47.5$ & 139.4 & 9.53 & 2.96 & 6.18 & 2.0 & 0.98 & $\ldots$ & 0.08 \\
\hline 364 & NGC 6186 & $16: 34: 25.49$ & $21: 32: 27.2$ & 162.8 & 10.31 & 2.15 & 3.83 & 2.7 & 0.56 & 1 & 0.30 \\
\hline 365 & MCG 9-27-053 & $16: 35: 15.41$ & $52: 46: 49.9$ & 129.2 & 9.99 & 2.30 & 5.34 & 4.0 & 0.45 & 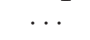 & 0.22 \\
\hline 366 & UGC 10514 & $16: 42: 23.66$ & $25: 05: 11.5$ & 100.5 & 9.81 & 1.69 & 5.74 & 8.1 & 0.41 & 8 & 0.32 \\
\hline 367 & IRAS $16435+2154$ & $16: 45: 40.68$ & 21:49:19.0 & 142.3 & 10.04 & 1.84 & 5.88 & 8.4 & 0.52 & $\ldots$ & 0.34 \\
\hline 368 & IC 4623 & $16: 51: 05.33$ & $22: 31: 38.6$ & 138.5 & 9.90 & 2.18 & 4.67 & 8.7 & 0.62 & $\cdots$ & 0.36 \\
\hline 369 & IRAS 16516+3030 & $16: 53: 37.18$ & $30: 26: 09.7$ & 306.1 & 10.66 & 1.97 & 5.34 & 1.5 & 0.85 & $\cdots$ & 0.26 \\
\hline
\end{tabular}


TABLE 2 - Continued

\begin{tabular}{|c|c|c|c|c|c|c|c|c|c|c|}
\hline SFRS & Name & $\begin{array}{l}\text { Positiona }^{\mathrm{a}} \\
(\mathrm{J} 2000)\end{array}$ & $\begin{array}{l}\text { Distance } \\
(\mathrm{Mpc})\end{array}$ & $\begin{array}{c}\log L_{60} \\
\left(\mathrm{~L}_{\odot}\right)\end{array}$ & $F_{100} / F_{60}$ & $\begin{array}{l}K_{s}-F_{60} \\
\text { (AB mag) }\end{array}$ & Weight & $\begin{array}{l}\text { Axial } \\
\text { Ratio }\end{array}$ & $\begin{array}{c}\mathrm{T} \\
\text { Type }\end{array}$ & $\begin{array}{c}\text { Extinction } \\
(B \text { mag })\end{array}$ \\
\hline
\end{tabular}

Note. - Morphological T types and B-band Milky Way extinction (in magnitudes, estimated from the $100 \mu \mathrm{m}$ dust maps) were taken from the PSCz catalog (Saunders et al. 2000), except as noted. Axial ratios were measured directly from the IRAC mosaics using SExtractor and are estimated to be accurate to $0.05(1 \sigma) . L_{60}$ is a monochromatic $60 \mu \mathrm{m}$ luminosity calculated for purposes of sample selection. The more useful $\mathrm{L}$ (TIR) is given in Table 7 . Weights are the ratio of the total number of PSCz galaxies within a given bin to the number of SFRS sample galaxies taken from that same bin, subject to the area restrictions noted in Sec. 2.

a Coordinates are based on IRAC imaging.

b Quality distances from R. B. Tully (priv. comm.) are given with $1 \sigma$ error estimates. All other distances are derived from the PSCz velocities after correcting for local bulk flow as described in Sec. 3.1. 
TABLE 4

Spitzer/IRAC Photometry For SFRS Galaxies

\begin{tabular}{|c|c|c|c|c|c|}
\hline SFRS & Name & $3.6 \mu \mathrm{m}$ & $4.5 \mu \mathrm{m}$ & $5.8 \mu \mathrm{m}$ & $8.0 \mu \mathrm{m}$ \\
\hline 1 & IC 486 & 12.58 & 12.71 & 12.37 & 11.81 \\
\hline 2 & IC 2217 & 13.06 & 13.44 & 11.95 & 10.78 \\
\hline 3 & NGC 2500 & 11.78 & 12.25 & 11.48 & 10.95 \\
\hline 4 & NGC 2512 & 12.25 & 12.64 & 11.67 & 10.63 \\
\hline 5 & MCG 6-18-009 & 13.14 & 13.50 & 12.80 & 11.51 \\
\hline 6 & MK 1212 & 13.65 & 13.90 & 12.85 & 11.37 \\
\hline 7 & IRAS $08072+1847$ & 13.81 & 13.35 & 12.11 & 10.94 \\
\hline 8 & NGC 2532 & $\ldots$ & 12.22 & $\ldots$ & 9.97 \\
\hline 9 & UGC 4261 & 14.07 & 14.48 & 13.31 & 12.17 \\
\hline 10 & NGC 2535 & 12.52 & 12.94 & 11.94 & 10.96 \\
\hline 11 & NGC 2543 & 11.92 & 12.32 & 11.67 & 10.72 \\
\hline 12 & NGC 2537 & 12.09 & 12.57 & 12.07 & 11.48 \\
\hline 13 & IC 2233 & 13.09 & 13.48 & 13.49 & 13.54 \\
\hline 14 & IC 2239 & 12.82 & 13.27 & 12.58 & 11.53 \\
\hline 15 & UGC 4286 & 13.27 & 13.69 & 12.68 & 11.63 \\
\hline 16 & UGC 4306 & 12.51 & 12.91 & 11.50 & 10.40 \\
\hline 17 & NGC 2552 & 12.78 & 13.27 & $14.01 \pm 0.04$ & 12.83 \\
\hline 18 & IC 2339 & 13.61 & 14.01 & 13.17 & 12.24 \\
\hline 19 & IRAS $08234+1054 \mathrm{~B}$ & 14.83 & 15.04 & 14.44 & 12.72 \\
\hline 20 & IRAS $08269+1514$ & 14.51 & 14.82 & 13.52 & 12.19 \\
\hline 21 & NGC 2604 & 12.87 & 13.23 & 12.83 & 11.76 \\
\hline 22 & NGC 2608 & 11.89 & 12.31 & 11.70 & 10.74 \\
\hline 23 & MK 92 & 13.86 & 14.22 & 12.73 & 11.56 \\
\hline 24 & NGC 2623 & 12.69 & 12.78 & 11.72 & 10.70 \\
\hline 25 & CGCG 120-018 & 13.68 & 13.99 & 12.73 & 11.48 \\
\hline 26 & NGC 2644 & 12.66 & 13.08 & 12.23 & 11.42 \\
\hline 27 & UGC 4572 & 13.04 & 13.52 & 12.70 & 11.73 \\
\hline 28 & UGC 4653 & 13.75 & 14.20 & 13.91 & 12.67 \\
\hline 29 & IRAS $08512+2727$ & 14.03 & 14.38 & 13.81 & 11.80 \\
\hline 30 & OJ 287 & 12.04 & 11.76 & 11.35 & 10.95 \\
\hline 31 & IRAS $08538+4256$ & 13.85 & 14.13 & 12.92 & 11.67 \\
\hline 32 & IRAS $08550+3908$ & 14.19 & 14.18 & 13.64 & 12.68 \\
\hline 33 & NGC 2718 & 11.90 & 12.37 & 11.48 & 10.55 \\
\hline 34 & NGC 2712 & 11.69 & 12.12 & 11.44 & 10.64 \\
\hline 35 & NGC 2719 & 13.99 & 14.41 & 13.76 & 12.98 \\
\hline 36 & IRAS $08572+3915$ & 12.26 & 11.16 & 10.13 & 9.49 \\
\hline 37 & IRAS $08579+3447$ & 14.21 & 14.44 & 13.87 & 12.15 \\
\hline 38 & NGC 2731 & 12.74 & 13.13 & 11.71 & 10.64 \\
\hline 39 & NGC 2730 & 13.10 & 13.55 & 12.78 & 11.73 \\
\hline 40 & IC 2431 & 13.81 & 14.16 & 13.36 & 11.88 \\
\hline 41 & NGC 2750 & 12.00 & 12.37 & 11.56 & 10.49 \\
\hline 42 & IC 2434 & 12.71 & 13.13 & 12.51 & 11.58 \\
\hline 43 & NGC 2761 & 12.86 & 13.18 & 11.85 & 10.56 \\
\hline 44 & NGC 2773 & 12.68 & 13.07 & 11.74 & 10.56 \\
\hline 45 & NGC 2776 & 11.65 & 11.98 & 11.09 & 10.05 \\
\hline 46 & NGC 2789 & 12.24 & 12.67 & 11.93 & 11.01 \\
\hline 47 & IRAS $09121+3908$ & 14.94 & 15.03 & 14.44 & 12.50 \\
\hline 48 & NGC 2824 & 12.66 & 13.14 & 12.89 & 12.45 \\
\hline 49 & IRAS $09184+4356$ & 13.77 & 14.09 & 13.02 & 11.54 \\
\hline 50 & CGCG 238-041 & 14.89 & 15.21 & 13.76 & 12.46 \\
\hline 51 & UGC 4985 & 13.92 & 14.27 & 13.06 & 11.87 \\
\hline 52 & NGC 2854 & 12.45 & 12.87 & 11.83 & 10.89 \\
\hline 53 & UGC 5046 & 12.84 & 13.22 & 11.92 & 10.79 \\
\hline 54 & UGC 5055 & 13.05 & 13.50 & 12.68 & 11.61 \\
\hline 55 & NGC 2893 & 12.65 & 13.12 & 12.18 & 11.15 \\
\hline 56 & MCG 3-24-062 & 13.05 & 13.46 & 12.21 & 11.11 \\
\hline 57 & CGCG 238-066 & 13.50 & 13.61 & 12.77 & 11.66 \\
\hline 58 & UGC 5097 & 13.18 & 13.56 & 12.08 & 10.96 \\
\hline 59 & CGCG 289-012 & 13.73 & 14.12 & 12.95 & 11.45 \\
\hline 60 & MCG 8-18-013 & 13.08 & 13.35 & 11.97 & 10.33 \\
\hline 61 & CGCG 181-068 & 13.20 & 13.60 & 12.57 & 11.48 \\
\hline 62 & NGC 2936 & 12.24 & 12.67 & 11.99 & 11.03 \\
\hline 63 & NGC 2955 & 12.40 & 12.82 & 11.93 & 10.88 \\
\hline 64 & CGCG $182-010$ & 13.49 & 13.72 & 12.71 & 11.28 \\
\hline 65 & UGC 5228 & 12.62 & 13.00 & 12.14 & 11.30 \\
\hline 66 & IRAS $09438+1141$ & 14.45 & 14.83 & 13.93 & 12.25 \\
\hline 67 & NGC 3015 & 12.76 & 13.16 & 11.95 & 10.73 \\
\hline 68 & MCG 2-25-039 & 13.32 & 13.71 & 12.30 & 11.12 \\
\hline 69 & NGC 3020 & 13.03 & 13.50 & 12.77 & 12.06 \\
\hline 70 & NGC 3049 & 12.38 & 12.84 & 11.92 & 11.16 \\
\hline 71 & NGC 3055 & 11.90 & 12.33 & 11.18 & 10.26 \\
\hline 72 & IC 2520 & 12.44 & 12.83 & 11.23 & 10.13 \\
\hline 73 & UGC 5403 & 12.68 & 13.10 & 11.99 & 10.94 \\
\hline 74 & UGC 5459 & 12.12 & 12.51 & 11.76 & 11.04 \\
\hline
\end{tabular}


Ashby et al.

TABLE $4-$ Continued

\begin{tabular}{|c|c|c|c|c|c|}
\hline SFRS & Name & $3.6 \mu \mathrm{m}$ & $4.5 \mu \mathrm{m}$ & $5.8 \mu \mathrm{m}$ & $8.0 \mu \mathrm{m}$ \\
\hline 75 & MCG 5-24-022 & 13.12 & 13.57 & 12.85 & 11.81 \\
\hline 76 & IC 2551 & 12.90 & 13.29 & 11.89 & 10.74 \\
\hline 77 & IRAS $10106+2745$ & 14.06 & 14.40 & 13.51 & 11.77 \\
\hline 78 & NGC 3162 & 11.79 & 12.27 & 11.26 & 10.33 \\
\hline 79 & IRAS $10120+1653$ & 15.98 & 16.06 & $15.72 \pm 0.04$ & 13.81 \\
\hline 80 & NGC 3190 & 10.10 & 10.60 & 10.50 & 10.20 \\
\hline 81 & IC 602 & 12.78 & 13.19 & 11.85 & 10.79 \\
\hline 82 & NGC 3191 & 13.30 & 13.66 & 12.46 & 11.21 \\
\hline 83 & NGC 3206 & 12.96 & 13.41 & $13.40 \pm 0.04$ & 12.62 \\
\hline 84 & UGC 5613 & 12.62 & 12.35 & 13.02 & 10.64 \\
\hline 85 & UGC 5644 & 13.02 & 13.48 & 12.98 & 12.07 \\
\hline 86 & NGC 3245 & 10.54 & 11.07 & 11.18 & 11.37 \\
\hline 87 & IRAS $10246+2042$ & 13.22 & 13.62 & 12.33 & 11.15 \\
\hline 88 & MCG 7-22-012 & 13.05 & 13.44 & 12.19 & 11.11 \\
\hline 89 & IRAS $10276+1119$ & 14.31 & 14.48 & 13.95 & 12.24 \\
\hline 90 & NGC 3265 & 12.82 & 13.27 & 12.20 & 11.18 \\
\hline 91 & UGC 5713 & 12.82 & 12.85 & 12.26 & 11.73 \\
\hline 92 & NGC 3274 & 13.16 & 13.58 & 13.32 & 13.03 \\
\hline 93 & UGC 5720 & 12.86 & 13.19 & 12.02 & 11.03 \\
\hline 94 & KUG $1031+351$ & 13.24 & 13.56 & 12.81 & 10.67 \\
\hline 95 & NGC 3306 & 12.63 & 13.05 & 11.67 & 10.58 \\
\hline 96 & NGC 3323 & 13.41 & 13.83 & 12.47 & 11.40 \\
\hline 97 & IC 2598 & 13.04 & 13.39 & 11.80 & 10.58 \\
\hline 98 & NGC 3338 & 10.91 & 11.39 & 10.67 & 9.81 \\
\hline 99 & NGC 3353 & 12.77 & 13.10 & 11.99 & 10.99 \\
\hline 100 & UGC 5881 & 12.88 & 13.18 & 12.48 & 11.60 \\
\hline 101 & NGC 3370 & 11.75 & 12.20 & 11.04 & 10.13 \\
\hline 102 & NGC 3381 & 12.55 & 12.97 & 11.99 & 11.17 \\
\hline 103 & UGC 5941 & 13.05 & 13.34 & 12.40 & 11.28 \\
\hline 104 & NGC 3413 & 12.24 & 12.67 & 12.24 & 11.80 \\
\hline 105 & NGC 3408 & 13.19 & 13.60 & 12.83 & 11.76 \\
\hline 106 & NGC 3430 & 13.96 & 14.06 & 13.58 & 12.64 \\
\hline 107 & CGCG 95-055 & 10.53 & 10.98 & 10.08 & 9.17 \\
\hline 108 & IRAS $10565+2448 \mathrm{~W}$ & 13.20 & 13.21 & 12.03 & 10.51 \\
\hline 109 & UGC 6074 & 12.97 & 13.38 & 12.52 & 11.47 \\
\hline 110 & NGC 3495 & 11.42 & 11.81 & 11.27 & 10.33 \\
\hline 111 & UGC 6103 & 12.95 & 13.30 & 12.06 & 10.92 \\
\hline 112 & MCG 7-23-019 & 13.37 & 13.63 & 12.29 & 10.92 \\
\hline 113 & UGC 6135 & 12.41 & 12.89 & 11.93 & 10.79 \\
\hline 114 & CGCG 241-078 & 13.67 & 13.90 & 12.74 & 11.60 \\
\hline 115 & IRAS $11069+2711$ & 15.60 & 15.94 & $15.26 \pm 0.04$ & 13.10 \\
\hline 116 & IC 676 & 12.19 & 12.64 & 11.83 & 10.99 \\
\hline 117 & IRAS $11102+3026$ & 14.23 & 14.48 & 13.42 & 12.23 \\
\hline 118 & IC 2637 & 12.64 & 12.96 & 11.94 & 10.86 \\
\hline 119 & MCG 9-19-013 & 14.17 & 14.48 & 13.40 & 11.77 \\
\hline 120 & 7ZW 384 & 14.52 & 14.77 & 14.31 & 12.43 \\
\hline 121 & IRAS $11167+5351$ & 13.60 & 13.31 & 12.82 & 11.91 \\
\hline 122 & NGC 3633 & 12.14 & 12.58 & 11.43 & 10.37 \\
\hline 123 & NGC 3652 & 12.68 & 13.08 & 12.05 & 11.22 \\
\hline 124 & NGC 3656 & 11.78 & 12.22 & 11.90 & 11.18 \\
\hline 125 & NGC 3659 & 12.61 & 13.03 & 11.97 & 11.07 \\
\hline 126 & NGC 3664 & 13.44 & 13.85 & $13.54 \pm 0.04$ & 12.84 \\
\hline 127 & NGC 3666 & 11.68 & 12.19 & 11.31 & 10.47 \\
\hline 128 & IC 691 & 12.72 & 12.82 & 11.84 & 10.84 \\
\hline 129 & NGC 3686 & 11.08 & 11.58 & 10.72 & 9.81 \\
\hline 130 & UGC 6469 & 13.44 & 13.83 & 12.61 & 11.41 \\
\hline 131 & NGC 3690 & 10.38 & 10.42 & 9.38 & 8.33 \\
\hline 132 & IC 698 & 12.42 & 12.82 & 11.50 & 10.33 \\
\hline 133 & IRAS $11267+1558$ & 16.15 & 16.07 & $15.75 \pm 0.04$ & 13.70 \\
\hline 134 & NGC 3705 & 10.50 & 10.99 & 10.49 & 9.84 \\
\hline 135 & MCG 3-29-061 & 13.40 & 13.78 & 12.58 & 11.49 \\
\hline 136 & NGC 3720 & 15.11 & 15.62 & $15.62 \pm 0.04$ & 15.16 \\
\hline 137 & NGC 3729 & 11.11 & 11.49 & 11.28 & 10.43 \\
\hline 138 & MCG 10-17-019 & 13.10 & 13.49 & 12.20 & 10.94 \\
\hline 139 & NGC 3758 & 12.03 & 12.06 & 11.54 & 10.92 \\
\hline 140 & UGC 6583 & 13.24 & 13.64 & 12.17 & 11.00 \\
\hline 141 & MCG 1-30-003 & 13.84 & 14.16 & 12.72 & 11.41 \\
\hline 142 & NGC 3769 & 11.68 & 12.13 & 11.37 & 10.59 \\
\hline 143 & NGC 3773 & 13.04 & 13.51 & 12.86 & 12.13 \\
\hline 144 & NGC 3781 & 12.85 & 13.12 & 12.24 & 11.22 \\
\hline 145 & UGC 6625 & 13.28 & 13.68 & 12.60 & 11.26 \\
\hline 146 & NGC 3808 & 13.44 & 13.78 & 12.41 & 11.22 \\
\hline 147 & NGC 3811 & 12.12 & 12.57 & 11.65 & 10.72 \\
\hline 148 & NGC 3822 & 11.87 & 12.23 & 11.23 & 10.12 \\
\hline 149 & UGC 6665 & 13.30 & 13.58 & 12.37 & 11.38 \\
\hline 150 & MCG 3-30-051 & 13.11 & 13.50 & 12.10 & 10.90 \\
\hline
\end{tabular}


TABLE 4 - Continued

\begin{tabular}{|c|c|c|c|c|c|}
\hline SFRS & Name & $3.6 \mu \mathrm{m}$ & $4.5 \mu \mathrm{m}$ & $5.8 \mu \mathrm{m}$ & $8.0 \mu \mathrm{m}$ \\
\hline 151 & NGC 3839 & 12.75 & 13.10 & 11.53 & 10.33 \\
\hline 152 & UGC 6732 & 12.33 & 12.52 & 12.11 & 11.50 \\
\hline 153 & IC 730 & 12.63 & 13.02 & 11.80 & 10.62 \\
\hline 154 & IC 732 & 13.77 & 14.12 & 12.91 & 11.71 \\
\hline 155 & NGC 3912 & 12.32 & 12.73 & 11.60 & 10.69 \\
\hline 156 & NGC 3928 & 12.11 & 12.53 & 11.68 & 10.73 \\
\hline 157 & NGC 3934 & 12.77 & 13.15 & 12.22 & 11.22 \\
\hline 158 & UGC 6865 & 12.45 & & 11.70 & 10.67 \\
\hline 159 & UGC 6901 & 12.78 & 13.18 & 11.86 & 10.67 \\
\hline 160 & CGCG 013-010 & 13.11 & 13.43 & 12.32 & 10.88 \\
\hline 161 & NGC 3991 & 12.30 & 12.65 & 11.58 & 10.70 \\
\hline 162 & NGC 4004 & 12.91 & 13.27 & 11.90 & 10.91 \\
\hline 163 & NGC 4014 & 11.95 & 12.38 & 11.41 & 10.41 \\
\hline 164 & NGC 4010 & 11.83 & 12.22 & 11.46 & 10.65 \\
\hline 165 & NGC 4018 & 12.65 & 13.04 & 11.72 & 10.66 \\
\hline 166 & NGC 4020 & 12.70 & 13.14 & 12.53 & 11.80 \\
\hline 167 & IRAS $11571+3003$ & 14.83 & 15.19 & 14.53 & 12.93 \\
\hline 168 & UGC 7017 & 12.49 & 12.87 & 11.37 & 10.25 \\
\hline 169 & UGC 7016 & 12.49 & 12.92 & 12.34 & 11.32 \\
\hline 170 & MCG 3-31-030 & 13.53 & 13.96 & 12.87 & 11.88 \\
\hline 171 & NGC 4062 & 10.69 & 11.19 & 10.55 & 9.84 \\
\hline 172 & NGC 4064 & 11.13 & 11.59 & 11.21 & 10.62 \\
\hline 173 & CGCG 098-059 & 12.86 & 13.13 & 11.84 & 10.65 \\
\hline 174 & NGC 4116 & 12.22 & 12.65 & 11.98 & 11.00 \\
\hline 175 & NGC 4136 & 11.78 & 12.28 & 11.65 & 10.97 \\
\hline 176 & NGC 4150 & 11.37 & 11.85 & 11.88 & 11.79 \\
\hline 177 & IRAS $12086+1441$ & 14.68 & 14.97 & 13.78 & 12.12 \\
\hline 178 & NGC 4162 & 11.81 & 12.28 & 11.48 & 10.53 \\
\hline 179 & NGC 4178 & 11.33 & 11.76 & 11.00 & 10.32 \\
\hline 180 & IRAS $12112+0305$ & 14.28 & 14.43 & 13.57 & 11.88 \\
\hline 181 & NGC 4189 & 11.53 & 12.01 & 11.23 & 10.20 \\
\hline 182 & NGC 4194 & 11.42 & 11.64 & 10.12 & 8.83 \\
\hline 183 & NGC 4204 & 13.47 & 13.63 & $14.34 \pm 0.05$ & 12.47 \\
\hline 184 & NGC 4207 & 11.86 & 12.26 & 11.31 & 10.41 \\
\hline 185 & UGC 7286 & 13.39 & 13.85 & 13.21 & 12.21 \\
\hline 186 & NGC 4234 & 12.70 & 13.13 & 12.24 & 11.22 \\
\hline 187 & NGC 4237 & 11.18 & 11.64 & 11.15 & 10.26 \\
\hline 188 & NGC 4244 & 10.31 & 10.72 & 10.66 & 10.48 \\
\hline 189 & NGC 4253 & 12.35 & 12.23 & 11.68 & 11.03 \\
\hline 190 & MCG 3-32-005 & 13.15 & 13.48 & 11.95 & 10.67 \\
\hline 191 & NGC 4290 & 11.65 & 12.08 & 11.49 & 10.64 \\
\hline 192 & NGC 4294 & 12.13 & 12.54 & 11.79 & 11.12 \\
\hline 193 & NGC 4314 & 10.26 & 10.79 & 10.87 & 10.83 \\
\hline 194 & NGC 4385 & 12.26 & 12.69 & 11.83 & 10.79 \\
\hline 195 & NGC 4395 & $10.13 \pm 0.05$ & $10.51 \pm 0.05$ & $10.28 \pm 0.05$ & $9.94 \pm 0.05$ \\
\hline 196 & NGC 4396 & 12.46 & 12.91 & 12.09 & 11.37 \\
\hline 197 & NGC 4412 & 12.16 & 12.60 & 11.72 & 10.78 \\
\hline 198 & NGC 4418 & 12.64 & 12.52 & 10.69 & 9.87 \\
\hline 199 & NGC 4420 & 12.03 & 12.43 & 11.40 & 10.48 \\
\hline 200 & NGC 4424 & 11.46 & 11.90 & 11.44 & 10.88 \\
\hline 201 & NGC 4435 & 10.36 & 11.06 & 11.18 & 11.26 \\
\hline 202 & NGC 4438 & 9.91 & 10.43 & 10.46 & 10.42 \\
\hline 203 & NGC 4448 & 10.42 & 11.01 & 11.01 & 10.77 \\
\hline 204 & $3 \mathrm{C} 273$ & 10.69 & 10.51 & 10.21 & 10.02 \\
\hline 205 & NGC 4470 & 12.56 & $13.04 \pm 0.04$ & 11.87 & 11.02 \\
\hline 206 & IRAS $12274+0018$ & 14.57 & 14.90 & 13.40 & 12.24 \\
\hline 207 & NGC 4491 & 12.42 & $\ldots$ & 12.69 & $\ldots$ \\
\hline 208 & NGC 4500 & 12.10 & 12.47 & 11.49 & 10.54 \\
\hline 209 & NGC 4495 & 12.40 & 12.77 & 11.57 & 10.57 \\
\hline 210 & IC 3476 & 12.70 & & 10.38 & $\ldots$ \\
\hline 211 & NGC 4509 & 14.63 & 15.01 & $14.92 \pm 0.04$ & 14.34 \\
\hline 212 & NGC 4519 & 12.09 & 12.51 & 11.96 & 10.71 \\
\hline 213 & NGC 4548 & 9.99 & 10.49 & 10.32 & 9.82 \\
\hline 214 & IRAS $12337+5044$ & 13.93 & 14.24 & 13.04 & 11.58 \\
\hline 215 & IC 3581 & 13.08 & 13.45 & 12.45 & 11.28 \\
\hline 216 & NGC 4592 & 12.19 & 12.63 & 12.36 & 11.54 \\
\hline 217 & NGC 4607 & 11.87 & 12.25 & 11.31 & 10.48 \\
\hline 218 & NGC 4625 & 12.40 & 12.87 & 12.11 & 11.22 \\
\hline 219 & NGC 4630 & 12.18 & 12.61 & 11.76 & 10.84 \\
\hline 220 & IC 3690 & 13.73 & 14.11 & 13.28 & 12.17 \\
\hline 221 & UGC 7905 & 13.77 & 14.14 & 12.86 & 11.81 \\
\hline 222 & MCG 5-30-069 & 13.27 & 13.64 & 12.29 & 11.15 \\
\hline 223 & IC 3721 & 12.69 & 13.10 & 12.18 & 11.07 \\
\hline 224 & NGC 4670 & 12.62 & 13.11 & 12.54 & 11.77 \\
\hline 225 & NGC 4675 & 12.96 & 13.35 & 12.37 & 11.34 \\
\hline 226 & MCG 7-26-051 & 12.99 & 13.29 & 11.98 & 10.64 \\
\hline
\end{tabular}


Ashby et al.

TABLE 4 - Continued

\begin{tabular}{|c|c|c|c|c|c|}
\hline SFRS & Name & $3.6 \mu \mathrm{m}$ & $4.5 \mu \mathrm{m}$ & $5.8 \mu \mathrm{m}$ & $8.0 \mu \mathrm{m}$ \\
\hline 227 & NGC 4689 & 10.93 & 11.36 & 10.71 & 9.84 \\
\hline 228 & NGC 4688 & 13.27 & 13.54 & $13.82 \pm 0.04$ & 12.53 \\
\hline 229 & NGC 4704 & 13.12 & 13.48 & 12.84 & 11.80 \\
\hline 230 & NGC 4701 & 12.24 & 12.66 & 11.66 & 10.75 \\
\hline 231 & IRAS $12468+3436$ & 15.88 & 15.94 & 15.47 & 13.18 \\
\hline 232 & IRAS $12470+1404$ & $\ldots$ & 15.42 & $\cdots$ & 13.02 \\
\hline 233 & MCG 8-23-097 & 13.30 & 13.61 & 12.57 & 11.36 \\
\hline 234 & NGC 4747 & 12.92 & 13.32 & 12.53 & 11.73 \\
\hline 235 & UGC 8017 & 12.52 & 12.95 & 11.80 & 10.66 \\
\hline 236 & NGC 4765 & 13.10 & 13.50 & 12.62 & 11.76 \\
\hline 237 & VCC 2096 & 14.02 & 14.29 & 13.69 & 11.81 \\
\hline 238 & UGC 8041 & 12.66 & 13.04 & $13.29 \pm 0.04$ & 11.94 \\
\hline 239 & UGC 8058 & 9.81 & 9.52 & 9.06 & 8.45 \\
\hline 240 & NGC 4837 & 12.96 & 13.32 & 12.27 & 11.18 \\
\hline 241 & UM 530 & 15.24 & 15.45 & $14.83 \pm 0.04$ & 12.91 \\
\hline 242 & NGC 4861 & $13.00 \pm 0.05$ & $13.35 \pm 0.05$ & $12.08 \pm 0.05$ & $12.75 \pm 0.05$ \\
\hline 243 & NGC 4868 & 11.89 & 12.33 & 11.31 & 10.33 \\
\hline 244 & NGC 4922 & 12.79 & 12.59 & 11.72 & 10.81 \\
\hline 245 & UGC 8179 & 13.13 & 13.54 & 12.88 & 11.58 \\
\hline 246 & NGC 5001 & 12.69 & 13.07 & 11.98 & 10.74 \\
\hline 247 & IC 856 & 13.20 & 13.60 & 12.31 & 11.24 \\
\hline 248 & UGC 8269 & 13.64 & 13.95 & 12.99 & 11.86 \\
\hline 249 & NGC 5014 & 12.48 & 12.91 & 11.92 & 10.99 \\
\hline 250 & NGC 5012 & 11.28 & 11.77 & 10.97 & 10.12 \\
\hline 251 & IRAS $13116+4508$ & 14.84 & 15.17 & $14.68 \pm 0.04$ & 12.75 \\
\hline 252 & IC 860 & 13.07 & 13.53 & 12.98 & 12.13 \\
\hline 253 & IRAS $13144+4508$ & $\ldots$ & 12.03 & $\ldots$ & 10.96 \\
\hline 254 & NGC 5060 & 12.60 & 13.01 & 12.09 & 11.00 \\
\hline 255 & UGC 8357 & 13.12 & 13.48 & 12.27 & 10.98 \\
\hline 256 & UGC 8361 & 13.01 & 13.39 & 12.26 & 11.08 \\
\hline 257 & IC 883 & 12.75 & 12.88 & 11.38 & 10.20 \\
\hline 258 & NGC 5100 & 12.69 & 12.93 & 12.03 & 10.92 \\
\hline 259 & NGC 5104 & 12.10 & 12.45 & 11.49 & 10.46 \\
\hline 260 & NGC 5107 & 13.53 & 13.97 & $13.81 \pm 0.04$ & 13.39 \\
\hline 261 & NGC 5112 & 12.11 & 12.61 & 11.96 & 11.22 \\
\hline 262 & NGC 5123 & 12.46 & 12.89 & 11.93 & 10.82 \\
\hline 263 & IRAS $13218+0552$ & 11.90 & 11.44 & 10.85 & 10.29 \\
\hline 264 & IRAS $13232+1731$ & 14.07 & 14.42 & 13.96 & 11.83 \\
\hline 265 & NGC 5147 & 11.98 & 12.41 & 11.43 & 10.68 \\
\hline 266 & NGC 5204 & 11.97 & 12.39 & 12.21 & 11.70 \\
\hline 267 & UGC 8502 & 13.97 & 14.28 & 13.10 & 11.78 \\
\hline 268 & UGC 8561 & 12.77 & 13.16 & 11.81 & 10.69 \\
\hline 269 & NGC 5230 & 12.25 & 12.70 & 12.00 & 10.86 \\
\hline 270 & IRAS $13349+2438$ & $\ldots$ & 11.51 & 10.59 & 10.12 \\
\hline 271 & NGC 5256 & 12.62 & 12.86 & 11.85 & 10.74 \\
\hline 272 & UGC 8626 & 13.24 & 13.66 & 12.87 & 11.76 \\
\hline 273 & NGC 5263 & 12.40 & 12.80 & 11.50 & 10.40 \\
\hline 274 & MCG 1-35-028 & 13.08 & 13.43 & 12.46 & 11.34 \\
\hline 275 & IC 910 & 13.34 & 13.48 & 12.40 & 11.23 \\
\hline 276 & MK 268 & 13.24 & 13.20 & 12.61 & 11.97 \\
\hline 277 & NGC 5278 & 12.56 & 13.00 & 12.42 & 11.45 \\
\hline 278 & NGC 5273 & 11.26 & 11.73 & 11.91 & 12.22 \\
\hline 279 & UGC 8685 & 13.13 & 13.49 & 12.69 & 11.53 \\
\hline 280 & UGC 8686 & 12.99 & 13.35 & 12.35 & 11.22 \\
\hline 281 & UGC 8696 & 12.69 & 12.37 & 11.40 & 10.49 \\
\hline 282 & NGC 5297 & 11.37 & 11.82 & 11.10 & 10.36 \\
\hline 283 & MK 796 & 12.67 & 12.56 & 11.82 & 10.93 \\
\hline 284 & IRAS $13446+1121$ & 12.76 & 12.66 & 11.89 & 10.96 \\
\hline 285 & NGC 5303 & 12.45 & 12.85 & 11.43 & 10.35 \\
\hline 286 & NGC 5313 & 11.33 & 11.77 & 10.91 & 9.99 \\
\hline 287 & MCG 3-35-034 & 13.25 & 13.62 & 12.89 & 11.62 \\
\hline 288 & NGC 5347 & 12.64 & 12.71 & 12.11 & 11.39 \\
\hline 289 & NGC 5350 & 11.07 & 11.42 & 11.07 & 10.24 \\
\hline 290 & NGC 5368 & 12.67 & 13.15 & 12.55 & 11.57 \\
\hline 291 & UGC 8827 & 12.59 & 12.93 & 11.96 & 10.89 \\
\hline 292 & UGC 8850 & 10.80 & 10.48 & 10.09 & 9.72 \\
\hline 293 & UGC 8856 & 13.61 & 13.93 & 12.47 & 11.19 \\
\hline 294 & NGC 5374 & 12.22 & 12.60 & 11.62 & 10.58 \\
\hline 295 & UGC 8902 & 12.45 & 12.91 & 12.38 & 11.46 \\
\hline 296 & NGC 5403 & 11.50 & 11.91 & 11.16 & 10.40 \\
\hline 297 & MCG 7-29-036 & 13.97 & 14.30 & 13.27 & 11.98 \\
\hline 298 & NGC 5414 & 13.19 & 13.55 & 12.30 & 11.23 \\
\hline 299 & MCG 5-33-046 & 13.35 & 13.70 & 12.72 & 11.61 \\
\hline 300 & NGC 5474 & 12.16 & 12.62 & 12.46 & 12.05 \\
\hline 301 & NGC 5480 & 11.03 & 11.45 & 10.19 & 9.21 \\
\hline 302 & MCG 6-31-070 & 13.40 & 13.72 & 12.54 & 11.23 \\
\hline
\end{tabular}


TABLE 4 - Continued

\begin{tabular}{|c|c|c|c|c|c|}
\hline SFRS & Name & $3.6 \mu \mathrm{m}$ & $4.5 \mu \mathrm{m}$ & $5.8 \mu \mathrm{m}$ & $8.0 \mu \mathrm{m}$ \\
\hline 303 & CGCG 074-129 & 13.37 & 13.29 & 12.27 & 11.34 \\
\hline 304 & NGC 5520 & 12.33 & 12.75 & 11.75 & 10.72 \\
\hline 305 & NGC 5515 & 12.29 & 12.69 & 11.85 & 10.80 \\
\hline 306 & NGC 5526 & 12.63 & 13.03 & 11.94 & 10.94 \\
\hline 307 & NGC 5522 & 12.33 & 12.78 & 12.03 & 11.04 \\
\hline 308 & NGC 5541 & 12.69 & 13.10 & 11.96 & 10.80 \\
\hline 309 & IC 4395 & 12.80 & 13.20 & 12.36 & 11.13 \\
\hline 310 & UGC 9165 & 12.49 & 12.86 & 11.57 & 10.44 \\
\hline 311 & MK 1490 & 13.35 & 13.58 & 12.27 & 10.94 \\
\hline 312 & NGC 5585 & 11.75 & 12.29 & 12.04 & 11.71 \\
\hline 313 & IC 4408 & 12.78 & 13.17 & 12.12 & 10.86 \\
\hline 314 & NGC 5584 & 11.83 & 12.25 & 11.54 & 10.75 \\
\hline 315 & NGC 5633 & 11.95 & 12.37 & 11.25 & 10.26 \\
\hline 316 & NGC 5660 & 11.80 & 12.22 & 11.33 & 10.25 \\
\hline 317 & NGC 5656 & 11.75 & 12.21 & 11.31 & 10.43 \\
\hline 318 & NGC 5657 & $\ldots$ & 13.25 & $\ldots$ & 11.33 \\
\hline 319 & CGCG 133-083 & 13.95 & 14.19 & 13.01 & 11.44 \\
\hline 320 & MCG 7-30-028 & 13.24 & 13.66 & 12.44 & 11.21 \\
\hline 321 & MCG 6-32-070 & 12.86 & 13.24 & 11.78 & 10.52 \\
\hline 322 & UGC 9412 & 10.93 & 10.71 & 10.36 & 9.62 \\
\hline 323 & NGC 5698 & 12.60 & 13.04 & 12.42 & 11.52 \\
\hline 324 & NGC 5691 & 12.18 & 12.65 & 11.61 & 10.74 \\
\hline 325 & MCG 9-24-035 & 13.00 & 13.43 & 12.79 & 11.72 \\
\hline 326 & MCG 9-24-038 & 13.46 & 13.80 & 12.63 & 11.20 \\
\hline 327 & UGC 9560 & 15.83 & 15.23 & 15.92 & 14.07 \\
\hline 328 & IC 1076 & 13.15 & 13.53 & 12.21 & 11.09 \\
\hline 329 & IRAS $14538+1730$ & 14.56 & 14.68 & 14.29 & 12.20 \\
\hline 330 & NGC 5795 & 12.21 & 12.64 & 11.52 & 10.47 \\
\hline 331 & UGC 9618 & 12.31 & 12.57 & 11.38 & 10.09 \\
\hline 332 & UGC 9639 & 12.69 & 12.96 & 12.00 & 10.67 \\
\hline 333 & MCG 6-33-022 & 13.42 & 13.71 & 12.62 & 11.10 \\
\hline 334 & NGC 5879 & 11.19 & 11.66 & 10.84 & 10.08 \\
\hline 335 & MCG 9-25-036 & 14.10 & 14.52 & 13.54 & 12.22 \\
\hline 336 & NGC 5899 & 11.12 & 11.50 & 10.75 & 9.89 \\
\hline 337 & NGC 5905 & 10.89 & 11.34 & 10.59 & 9.66 \\
\hline 338 & MK 848 & 13.31 & 13.44 & 12.20 & 10.69 \\
\hline 339 & IC 4553 & 12.11 & 12.25 & 11.08 & 10.07 \\
\hline 340 & UGC 9922 & 13.41 & 13.79 & 12.28 & 11.14 \\
\hline 341 & IC 4567 & 12.38 & 12.79 & 11.72 & 10.62 \\
\hline 342 & MCG 4-37-016 & 13.61 & 13.96 & 13.09 & 12.03 \\
\hline 343 & NGC 5975 & 12.30 & 12.73 & 11.79 & 10.75 \\
\hline 344 & NGC 5980 & 11.81 & 12.22 & 11.20 & 10.22 \\
\hline 345 & NGC 5992 & 13.45 & 13.79 & 12.38 & 11.08 \\
\hline 346 & NGC 5996 & 12.33 & 12.72 & 11.61 & 10.65 \\
\hline 347 & IRAS $15519+3537$ & 14.55 & 14.86 & 14.28 & 12.00 \\
\hline 348 & UGC 10099 & 13.59 & 13.94 & 12.66 & 11.32 \\
\hline 349 & MCG 5-38-006 & 12.93 & 13.37 & 12.24 & 11.15 \\
\hline 350 & UGC 10120 & 12.55 & 12.51 & 12.13 & 11.61 \\
\hline 351 & NGC 6027A & 12.72 & 13.18 & 13.06 & 12.54 \\
\hline 352 & NGC 6040 & 13.12 & 13.52 & 13.32 & 12.36 \\
\hline 353 & UGC 10200 & 13.81 & 14.10 & 13.15 & 12.35 \\
\hline 354 & IRAS $16052+5334$ & 14.43 & 14.63 & 14.10 & 11.84 \\
\hline 355 & IRAS $16053+1836$ & 13.40 & 13.73 & 12.60 & 11.21 \\
\hline 356 & NGC 6090 & 12.84 & 13.14 & 11.62 & 10.34 \\
\hline 357 & UGC 10273 & 13.88 & 14.24 & 12.93 & 11.81 \\
\hline 358 & IRAS $16150+2233$ & 14.75 & 14.55 & 13.91 & 12.77 \\
\hline 359 & UGC 10322 & 12.70 & 13.11 & 11.78 & 10.67 \\
\hline 360 & NGC 6120 & 12.62 & 12.98 & 11.64 & 10.31 \\
\hline 361 & MCG 3-42-004 & 13.43 & 13.79 & 13.02 & 11.65 \\
\hline 362 & UGC 10407 & 13.78 & 14.13 & 12.73 & 11.55 \\
\hline 363 & IRAS $16320+3922$ & 13.40 & 13.86 & 13.20 & 12.15 \\
\hline 364 & NGC 6186 & 12.00 & 12.43 & 11.73 & 10.78 \\
\hline 365 & MCG 9-27-053 & 13.71 & 14.05 & 13.10 & 11.94 \\
\hline 366 & UGC 10514 & 13.36 & 13.74 & 12.27 & 11.10 \\
\hline 367 & IRAS $16435+2154$ & 14.00 & 14.33 & 12.94 & 11.61 \\
\hline 368 & IC 4623 & 13.36 & 13.74 & 12.69 & 11.43 \\
\hline 369 & IRAS $16516+3030$ & 13.91 & 14.18 & 13.60 & 11.48 \\
\hline
\end{tabular}

Note. - Table 4 is published in its entirety in the electronic edition of Publications of the Astronomical Society of the Pacific. All magnitudes are expressed on the AB system. Where uncertainties are not given, they are dominated by the uncertainty in the cryogenic IRAC absolute flux calibration $(3 \%, 1 \sigma)$. 
TABLE 5

1.4 GHz Flux Measurements for SFRS Galaxies

\begin{tabular}{|c|c|c|c|c|c|c|}
\hline SFRS & Name & $\begin{array}{c}\text { Position }^{\mathrm{a}} \\
(\mathrm{J} 2000)\end{array}$ & $\begin{array}{l}\text { Offset }^{\mathrm{b}} \\
(\operatorname{arcsec})\end{array}$ & $\begin{array}{c}\mathrm{F}(1.4 \mathrm{GHz}) \\
(\mathrm{mJy})\end{array}$ & References ${ }^{\mathrm{c}}$ & Remarks $^{\mathrm{d}}$ \\
\hline 1 & IC 486 & $08: 00: 20.98+26: 36: 50.2$ & 1.5 & $10.2 \pm 0.5$ & 1 & \\
\hline 2 & IC 2217 & $08: 00: 49.89+27: 30: 01.9$ & 2.2 & $18.8 \pm 0.7$ & 1 & \\
\hline 3 & NGC 2500 & $08: 01: 52.41+50: 44: 25.6$ & 14.0 & $14.7 \pm 3.2$ & 1 & $\mathrm{E}^{*}$ \\
\hline 4 & NGC 2512 & $08: 03: 07.86+23: 23: 31.6$ & 1.1 & $18.6 \pm 0.7$ & 1 & \\
\hline 5 & MCG 6-18-009 & $08: 03: 28.82+33: 27: 44.4$ & 1.5 & $17.9 \pm 0.7$ & 1 & \\
\hline 6 & MK 1212 & $08: 07: 05.61+27: 07: 33.8$ & 1.2 & $12.4 \pm 0.6$ & 1 & \\
\hline 7 & IRAS $08072+1847$ & $08: 10: 07.18+18: 38: 17.8$ & 2.4 & $6.8 \pm 0.4$ & 1 & \\
\hline 8 & NGC 2532 & $08: 10: 15.43+33: 57: 25.9$ & 3.9 & $46.5 \pm 2.1$ & 1 & \\
\hline 9 & UGC 4261 & $08: 10: 56.46+36: 49: 47.3$ & 6.7 & $8.2 \pm 0.5$ & 1 & \\
\hline 10 & NGC 2535 & $08: 11: 13.63+25: 12: 27.1$ & 13.9 & $18.2 \pm 1.9$ & 1,2 & $\mathrm{E}^{*}$ \\
\hline 11 & NGC 2543 & $08: 12: 57.84+36: 15: 15.2$ & 1.7 & $12.2 \pm 0.9$ & 1 & \\
\hline 12 & NGC 2537 & $08: 13: 14.03+45: 59: 37.3$ & 17.1 & $10.5 \pm 1.7$ & 1 & $\mathrm{E}^{*}$ \\
\hline 13 & IC 2233 & $08: 13: 58.82+45: 44: 43.7$ & $\ldots$ & $<2.0$ & 2 & \\
\hline 14 & IC 2239 & $08: 14: 07.01+23: 51: 59.7$ & 3.1 & $12.5 \pm 0.6$ & 1 & \\
\hline 15 & UGC 4286 & $08: 14: 16.50+18: 26: 26.0$ & $\cdots$ & $3.9 \pm 0.6$ & 3 & $\mathrm{D}$ \\
\hline 16 & UGC 4306 & $08: 17: 36.83+35: 26: 45.0$ & 6.4 & $27.1 \pm 0.9$ & 1 & \\
\hline 17 & NGC 2552 & $08: 19: 19.58+50: 00: 20.8$ & $\ldots$ & $<0.4$ & 3 & \\
\hline 18 & IC 2339 & $08: 23: 33.04+21: 20: 27.0$ & 29.4 & $6.0 \pm 0.9$ & 2 & $\mathrm{D}, \mathrm{E}^{*}$ \\
\hline 19 & IRAS $08234+1054 \mathrm{~B}$ & $08: 26: 07.73+10: 44: 52.5$ & 2.8 & $8.9 \pm 0.5$ & 1 & \\
\hline 20 & IRAS $08269+1514$ & $08: 29: 45.30+15: 04: 38.1$ & 2.1 & $6.5 \pm 0.5$ & 1 & \\
\hline 21 & NGC 2604 & $08: 33: 22.54+29: 32: 14.9$ & 9.1 & $9.4 \pm 1.0$ & 1 & \\
\hline 22 & NGC 2608 & $08: 35: 17.19+28: 28: 23.2$ & 7.8 & $15.2 \pm 1.4$ & 1 & \\
\hline 23 & MK 92 & $08: 35: 40.17+46: 29: 33.8$ & 6.1 & $24.3 \pm 1.1$ & 1 & \\
\hline 24 & NGC 2623 & $08: 38: 24.10+25: 45: 16.3$ & 1.3 & $95.7 \pm 2.9$ & 1 & \\
\hline 25 & CGCG $120-018$ & $08: 39: 50.61+23: 08: 37.5$ & 2.5 & $12.3 \pm 0.5$ & 1 & \\
\hline 26 & NGC 2644 & $08: 41: 32.07+04: 58: 53.3$ & 5.3 & $11.8 \pm 1.0$ & 1 & \\
\hline 27 & UGC 4572 & $08: 45: 37.83+36: 56: 04.4$ & 0.5 & $8.1 \pm 0.5$ & 1 & \\
\hline 28 & UGC 4653 & $08: 53: 54.40+35: 08: 56.4$ & 12.6 & $38.0 \pm 1.5$ & 1 & \\
\hline 29 & IRAS $08512+2727$ & $08: 54: 16.83+27: 16: 01.0$ & 1.7 & $8.1 \pm 0.5$ & 1 & \\
\hline 30 & OJ 287 & $08: 54: 48.87+20: 06: 30.7$ & 0.1 & $1511.8 \pm 5.4$ & 1 & \\
\hline 31 & IRAS $08538+4256$ & $08: 57: 10.48+42: 45: 24.2$ & 2.1 & $21.0 \pm 0.7$ & 1 & \\
\hline 32 & IRAS $08550+3908$ & $08: 58: 13.98+38: 56: 30.8$ & 2.9 & $24.0 \pm 0.8$ & 1 & \\
\hline 33 & NGC 2718 & $08: 58: 50.63+06: 17: 33.7$ & 2.7 & $29.6 \pm 1.6$ & 1 & \\
\hline 34 & NGC 2712 & $08: 59: 30.62+44: 54: 48.9$ & 1.9 & $12.4 \pm 1.0$ & 1 & \\
\hline 35 & NGC 2719 & $09: 00: 15.72+35: 43: 28.6$ & 10.8 & $29.7 \pm 1.6$ & 1 & $\mathrm{~B}$ \\
\hline 36 & IRAS $08572+3915$ & $09: 00: 25.58+39: 03: 52.8$ & 2.6 & $4.3 \pm 0.4$ & 1 & \\
\hline 37 & IRAS $08579+3447$ & $09: 01: 05.81+34: 35: 28.5$ & 0.3 & $24.4 \pm 0.8$ & 1 & \\
\hline 38 & NGC 2731 & $09: 02: 08.38+08: 18: 02.7$ & 3.3 & $23.9 \pm 1.1$ & 1 & \\
\hline 39 & NGC 2730 & $09: 02: 15.80+16: 50: 15.4$ & 2.5 & $8.9 \pm 1.3$ & 1 & \\
\hline 40 & IC 2431 & $09: 04: 34.84+14: 35: 38.8$ & 6.6 & $104.1 \pm 3.7$ & 1 & \\
\hline 41 & NGC 2750 & $09: 05: 47.77+25: 26: 21.2$ & 6.6 & $25.0 \pm 1.7$ & 1 & \\
\hline 42 & IC 2434 & $09: 07: 15.96+37: 12: 55.8$ & 1.3 & $21.2 \pm 1.0$ & 1 & \\
\hline 43 & NGC 2761 & $09: 07: 30.91+18: 26: 04.7$ & 1.1 & $28.0 \pm 0.9$ & 1 & \\
\hline 44 & NGC 2773 & $09: 09: 44.15+07: 10: 25.6$ & 0.1 & $22.6 \pm 0.8$ & 1 & \\
\hline 45 & NGC 2776 & $09: 12: 14.26+44: 57: 22.8$ & 6.0 & $31.1 \pm 2.5$ & 1 & \\
\hline 46 & NGC 2789 & $09: 14: 59.62+29: 43: 49.1$ & 0.7 & $18.3 \pm 0.7$ & 1 & \\
\hline 47 & IRAS 09121+3908 & $09: 15: 22.15+38: 56: 35.0$ & $\ldots$ & $5.4 \pm 0.7$ & 2 & $\mathrm{D}^{*}$ \\
\hline 48 & NGC 2824 & $09: 19: 02.27+26: 16: 12.7$ & 0.9 & $8.9 \pm 0.5$ & 1 & \\
\hline 49 & IRAS $09184+4356$ & $09: 21: 38.62+43: 43: 36.0$ & 2.2 & $9.8 \pm 0.5$ & 1 & \\
\hline 50 & CGCG 238-041 & $09: 22: 25.58+47: 14: 40.8$ & 3.0 & $8.4 \pm 0.9$ & 1 & \\
\hline 51 & UGC 4985 & $09: 22: 37.65+21: 57: 28.9$ & 4.2 & $6.1 \pm 0.5$ & 1 & \\
\hline 52 & NGC 2854 & $09: 24: 02.92+49: 12: 13.4$ & 0.9 & $18.8 \pm 1.0$ & 1 & \\
\hline 53 & UGC 5046 & $09: 28: 06.54+17: 11: 46.8$ & 1.7 & $22.1 \pm 0.8$ & 1 & \\
\hline 54 & UGC 5055 & $09: 30: 11.76+55: 51: 09.9$ & 1.2 & $11.4 \pm 0.5$ & 1 & \\
\hline 55 & NGC 2893 & $09: 30: 17.00+29: 32: 25.6$ & 1.7 & $7.4 \pm 0.5$ & 1 & \\
\hline 56 & MCG 3-24-062 & $09: 30: 23.02+19: 28: 11.7$ & 2.5 & $11.5 \pm 0.5$ & 1 & \\
\hline 57 & CGCG 238-066 & $09: 31: 06.67+49: 04: 48.5$ & 1.7 & $8.9 \pm 0.5$ & 1 & \\
\hline 58 & UGC 5097 & $09: 34: 10.68+00: 14: 31.9$ & 0.8 & $27.7 \pm 1.2$ & 1 & \\
\hline 59 & CGCG 289-012 & $09: 36: 31.92+59: 23: 53.8$ & 0.6 & $13.6 \pm 0.6$ & 1 & \\
\hline 60 & MCG 8-18-013 & $09: 36: 36.98+48: 28: 21.2$ & 7.1 & $34.1 \pm 1.5$ & 1 & \\
\hline 61 & CGCG 181-068 & $09: 37: 19.34+33: 49: 27.2$ & 2.0 & $12.6 \pm 0.6$ & 1 & \\
\hline 62 & NGC 2936 & $09: 37: 44.02+02: 45: 33.4$ & 5.8 & $29.2 \pm 1.5$ & 1 & \\
\hline 63 & NGC 2955 & $09: 41: 16.78+35: 52: 58.1$ & 2.9 & $12.9 \pm 0.6$ & 1 & \\
\hline 64 & CGCG 182-010 & $09: 45: 15.06+34: 42: 46.5$ & 3.0 & $12.1 \pm 0.5$ & 1 & \\
\hline 65 & UGC 5228 & $09: 46: 03.60+01: 40: 06.1$ & ... & $13.7 \pm 2.1$ & 2 & $\mathrm{D}, \mathrm{E}^{*}$ \\
\hline 66 & IRAS $09438+1141$ & $09: 46: 32.33+11: 27: 20.5$ & 3.6 & $9.6 \pm 1.1$ & 1 & \\
\hline 67 & NGC 3015 & $09: 49: 22.97+01: 08: 43.4$ & 0.7 & $20.5 \pm 0.8$ & 1 & \\
\hline 68 & MCG 2-25-039 & $09: 49: 37.05+09: 00: 18.2$ & 1.1 & $14.7 \pm 0.6$ & 1 & \\
\hline 69 & NGC 3020 & $09: 50: 06.65+12: 48: 49.0$ & $\ldots$ & $44.0 \pm 4.0$ & 2 & $\mathrm{E}$ \\
\hline 70 & NGC 3049 & $09: 54: 49.49+09: 16: 13.6$ & 2.6 & $9.1 \pm 1.1$ & 1 & \\
\hline 71 & NGC 3055 & $09: 55: 17.99+04: 16: 11.2$ & 1.6 & $36.6 \pm 1.8$ & 1 & \\
\hline 72 & IC 2520 & $09: 56: 20.22+27: 13: 39.9$ & 1.6 & $24.1 \pm 1.4$ & 1 & \\
\hline 73 & UGC 5403 & $10: 02: 35.57+19: 10: 36.7$ & 0.4 & $11.8 \pm 0.5$ & 1 & \\
\hline
\end{tabular}


TABLE $5-$ Continued

\begin{tabular}{|c|c|c|c|c|c|c|}
\hline SFRS & Name & $\begin{array}{l}\text { Position }^{\mathrm{a}} \\
(\mathrm{J} 2000)\end{array}$ & $\begin{array}{l}\text { Offset }^{\mathrm{b}} \\
(\operatorname{arcsec})\end{array}$ & 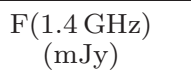 & References $^{\mathrm{c}}$ & Remarks $^{\mathrm{d}}$ \\
\hline 74 & UGC 5459 & $10: 08: 08.87+53: 05: 12.2$ & 15.3 & $16.6 \pm 2.0$ & 1,2 & $\mathrm{E}^{*}$ \\
\hline 75 & MCG 5-24-022 & $10: 10: 02.78+32: 04: 09.1$ & 8.6 & $5.6 \pm 0.4$ & 1 & \\
\hline 76 & IC 2551 & $10: 10: 40.30+24: 24: 50.0$ & 1.0 & $25.8 \pm 0.9$ & 1 & \\
\hline 77 & IRAS $10106+2745$ & $10: 13: 29.51+27: 30: 41.0$ & 0.9 & $8.1 \pm 0.5$ & 1 & \\
\hline 78 & NGC 3162 & $10: 13: 31.68+22: 44: 17.4$ & 2.5 & $18.0 \pm 1.9$ & 1 & \\
\hline 79 & IRAS $10120+1653$ & $10: 14: 47.95+16: 38: 48.6$ & 1.6 & $9.7 \pm 0.5$ & 1 & \\
\hline 80 & NGC 3190 & $10: 18: 06.60+21: 49: 27.4$ & $\cdots$ & $5.0 \pm 2.0$ & 2 & $\mathrm{D}, \mathrm{E}^{*}$ \\
\hline 81 & IC 602 & $10: 18: 19.75+07: 02: 55.0$ & 2.6 & $29.7 \pm 1.5$ & 1 & \\
\hline 82 & NGC 3191 & $10: 19: 05.05+46: 27: 16.9$ & 2.3 & $12.8 \pm 0.6$ & 1 & \\
\hline 83 & NGC 3206 & $10: 21: 43.30+56: 55: 16.0$ & $\cdots$ & $0.8 \pm 0.2$ & 3 & $\mathrm{E}^{*}$ \\
\hline 84 & UGC 5613 & $10: 23: 32.53+52: 20: 31.5$ & 0.2 & $49.6 \pm 1.5$ & 1 & \\
\hline 85 & UGC 5644 & $10: 25: 46.10+13: 43: 04.1$ & 4.0 & $13.1 \pm 0.9$ & 1 & \\
\hline 86 & NGC 3245 & $10: 27: 18.18+28: 30: 27.2$ & 3.1 & $6.7 \pm 0.5$ & 1 & \\
\hline 87 & IRAS $10246+2042$ & $10: 27: 25.76+20: 26: 54.8$ & 3.7 & $12.3 \pm 0.9$ & 1 & \\
\hline 88 & MCG 7-22-012 & $10: 30: 11.16+43: 21: 37.9$ & 2.9 & $12.1 \pm 0.5$ & 1 & \\
\hline 89 & IRAS $10276+1119$ & $10: 30: 14.81+11: 04: 11.8$ & 4.1 & $5.8 \pm 0.4$ & 1 & \\
\hline 90 & NGC 3265 & $10: 31: 06.87+28: 47: 51.9$ & 4.2 & $10.1 \pm 0.9$ & 1 & \\
\hline 91 & UGC 5713 & $10: 31: 41.38+25: 59: 16.6$ & $\cdots$ & $<1.0$ & 2 & $\mathrm{D}$ \\
\hline 92 & NGC 3274 & $10: 32: 17.49+27: 40: 10.5$ & 4.4 & $4.3 \pm 0.7$ & 1 & \\
\hline 93 & UGC 5720 & $10: 32: 31.98+54: 24: 02.4$ & 1.6 & $16.6 \pm 0.6$ & 1 & \\
\hline 94 & KUG $1031+351$ & $10: 34: 02.44+34: 52: 10.0$ & 0.6 & $38.3 \pm 1.5$ & 1 & \\
\hline 95 & NGC 3306 & $10: 37: 10.26+12: 39: 08.8$ & 0.7 & $23.3 \pm 0.8$ & 1 & \\
\hline 96 & NGC 3323 & $10: 39: 39.05+25: 19: 20.3$ & 1.6 & $13.4 \pm 0.6$ & 1 & \\
\hline 97 & IC 2598 & $10: 39: 42.38+26: 43: 38.4$ & 0.2 & $27.2 \pm 0.9$ & 1 & \\
\hline 98 & NGC 3338 & $10: 42: 06.58+13: 44: 47.5$ & ... & $42.7 \pm 4.0$ & 2 & $\mathrm{D}, \mathrm{E}^{*}$ \\
\hline 99 & NGC 3353 & $10: 45: 22.26+55: 57: 40.9$ & 2.0 & $15.5 \pm 0.9$ & 1 & \\
\hline 100 & UGC 5881 & $10: 46: 42.55+25: 55: 52.7$ & 0.9 & $10.7 \pm 0.5$ & 1 & \\
\hline 101 & NGC 3370 & $10: 47: 04.14+17: 16: 30.3$ & 5.3 & $24.9 \pm 1.9$ & 1 & \\
\hline 102 & NGC 3381 & $10: 48: 24.96+34: 42: 30.4$ & 10.8 & $7.6 \pm 0.6$ & 2 & $\mathrm{E}^{*}$ \\
\hline 103 & UGC 5941 & $10: 50: 21.60+41: 27: 50.5$ & $\ldots$ & $12.5 \pm 1.0$ & 2 & $\mathrm{D}, \mathrm{E}^{*}$ \\
\hline 104 & NGC 3413 & $10: 51: 20.48+32: 46: 01.7$ & 4.2 & $5.1 \pm 0.5$ & 1 & \\
\hline 105 & NGC 3408 & $10: 52: 11.96+58: 26: 18.4$ & 2.4 & $6.1 \pm 0.5$ & 1 & \\
\hline 106 & NGC 3430 & $10: 52: 11.18+32: 57: 02.2$ & 2.8 & $32.7 \pm 2.2$ & 1 & * \\
\hline 107 & CGCG 95-055 & $10: 52: 50.51+16: 59: 07.6$ & 3.3 & $10.5 \pm 0.5$ & 1 & $\mathrm{D}^{*}$ \\
\hline 108 & IRAS $10565+2448 \mathrm{~W}$ & $10: 59: 18.20+24: 32: 35.0$ & 1.1 & $57.0 \pm 2.1$ & 1 & $\mathrm{E}$ \\
\hline 109 & UGC 6074 & $10: 59: 58.17+50: 54: 11.7$ & 1.3 & $8.8 \pm 0.5$ & 1 & \\
\hline 110 & NGC 3495 & $11: 01: 16.05+03: 37: 42.6$ & $\cdots$ & $25.8 \pm 2.0$ & 2 & $\mathrm{E}^{*}$ \\
\hline 111 & UGC 6103 & $11: 01: 58.92+45: 13: 43.3$ & 2.5 & $14.6 \pm 0.6$ & 1 & \\
\hline 112 & MCG 7-23-019 & $11: 03: 53.89+40: 50: 59.9$ & 4.7 & $36.4 \pm 1.2$ & 1 & \\
\hline 113 & UGC 6135 & $11: 04: 36.82+45: 07: 31.4$ & 1.6 & $17.4 \pm 1.0$ & 1 & \\
\hline 114 & CGCG 241-078 & $11: 06: 37.37+46: 02: 19.2$ & 0.4 & $16.3 \pm 0.6$ & 1 & \\
\hline 115 & IRAS 11069+2711 & $11: 09: 38.90+26: 54: 56.0$ & $\cdots$ & $2.4 \pm 0.8$ & 2 & $\mathrm{D}^{*}$ \\
\hline 116 & IC 676 & $11: 12: 39.82+09: 03: 22.5$ & 1.5 & $9.7 \pm 0.5$ & 1 & \\
\hline 117 & IRAS $11102+3026$ & $11: 12: 57.50+30: 10: 30.0$ & 2.3 & $10.5 \pm 0.5$ & 1 & \\
\hline 118 & IC 2637 & $11: 13: 49.71+09: 35: 10.5$ & 0.7 & $42.2 \pm 1.3$ & 1 & \\
\hline 119 & MCG 9-19-013 & $11: 14: 49.37+50: 19: 22.5$ & $\ldots$ & $6.2 \pm 1.2$ & 2 & $\mathrm{D}^{*}$ \\
\hline 120 & 7ZW 384 & $11: 16: 54.03+59: 31: 48.7$ & $\cdots$ & $17.8 \pm 1.8$ & 2 & $\mathrm{D}^{*}$ \\
\hline 121 & IRAS $11167+5351$ & $11: 19: 34.31+53: 35: 18.3$ & 2.7 & $16.1 \pm 0.6$ & 1 & \\
\hline 122 & NGC 3633 & $11: 20: 26.27+03: 35: 08.5$ & 1.0 & $19.4 \pm 1.0$ & 1 & \\
\hline 123 & NGC 3652 & $11: 22: 38.66+37: 45: 55.3$ & 4.4 & $22.9 \pm 1.5$ & 1 & \\
\hline 124 & NGC 3656 & $11: 23: 38.43+53: 50: 32.9$ & 2.3 & $19.8 \pm 0.7$ & 1 & \\
\hline 125 & NGC 3659 & $11: 23: 45.45+17: 49: 03.2$ & 4.1 & $12.8 \pm 1.4$ & 1 & \\
\hline 126 & NGC 3664 & $11: 24: 25.16+03: 19: 32.8$ & 13.5 & $9.4 \pm 1.9$ & 2 & $\mathrm{E}^{*}$ \\
\hline 127 & NGC 3666 & $11: 24: 26.28+11: 20: 27.9$ & 5.2 & $16.8 \pm 1.7$ & 1 & \\
\hline 128 & IC 691 & $11: 26: 44.48+59: 09: 00.2$ & 19.4 & $37.8 \pm 1.8$ & 1 & $\mathrm{~B}, \mathrm{E}^{*}$ \\
\hline 129 & NGC 3686 & $11: 27: 43.99+17: 13: 29.5$ & 2.5 & $15.8 \pm 2.0$ & 1 & \\
\hline 130 & UGC 6469 & $11: 28: 17.77+02: 39: 14.7$ & 1.0 & $18.1 \pm 1.0$ & 1 & \\
\hline 131 & NGC 3690 & $11: 28: 32.61+58: 33: 46.8$ & 9.8 & $677.1 \pm 5.4$ & 1 & \\
\hline 132 & IC 698 & $11: 29: 03.78+09: 06: 42.7$ & 1.1 & $31.1 \pm 1.0$ & 1 & \\
\hline 133 & IRAS $11267+1558$ & $11: 29: 24.80+15: 41: 41.9$ & 1.6 & $3.1 \pm 0.4$ & 1 & \\
\hline 134 & NGC 3705 & $11: 30: 08.19+09: 16: 26.4$ & $\cdots$ & $20.6 \pm 0.7$ & 2 & $\mathrm{E}^{*}$ \\
\hline 135 & MCG 3-29-061 & $11: 31: 03.67+20: 14: 08.3$ & 0.4 & $8.2 \pm 0.5$ & 1 & \\
\hline 136 & NGC 3720 & $11: 32: 21.76+00: 48: 14.9$ & 2.5 & $11.1 \pm 0.6$ & 1 & \\
\hline 137 & NGC 3729 & $11: 33: 49.36+53: 07: 33.5$ & 1.6 & $21.0 \pm 1.5$ & 1 & \\
\hline 138 & MCG 10-17-019 & $11: 35: 24.67+57: 38: 59.7$ & 1.0 & $14.1 \pm 0.6$ & 1 & \\
\hline 139 & NGC 3758 & $11: 36: 29.17+21: 35: 49.1$ & 4.1 & $10.0 \pm 0.5$ & 1 & \\
\hline 140 & UGC 6583 & $11: 36: 54.25+19: 58: 19.4$ & 2.0 & $18.6 \pm 0.7$ & 1 & \\
\hline 141 & MCG 1-30-003 & $11: 37: 06.84+02: 50: 44.7$ & $\ldots$ & $9.7 \pm 0.5$ & 2 & $\mathrm{D}^{*}$ \\
\hline 142 & NGC 3769 & $11: 37: 44.27+47: 53: 27.9$ & 7.4 & $19.7 \pm 1.9$ & 1 & \\
\hline 143 & NGC 3773 & $11: 38: 12.90+12: 06: 45.5$ & 2.6 & $5.8 \pm 0.5$ & 1 & \\
\hline 144 & NGC 3781 & $11: 39: 03.72+26: 21: 43.0$ & 1.0 & $50.1 \pm 1.6$ & 1 & \\
\hline 145 & UGC 6625 & $11: 39: 47.55+19: 55: 58.5$ & 1.8 & $17.4 \pm 1.3$ & 1 & \\
\hline 146 & NGC 3808 & $11: 40: 44.53+22: 26: 42.9$ & 6.3 & $25.5 \pm 1.3$ & 1 & \\
\hline 147 & NGC 3811 & $11: 41: 13.17+47: 41: 45.7$ & $\ldots$ & $16.0 \pm 2.0$ & 2 & $\mathrm{D}, \mathrm{E}^{*}$ \\
\hline
\end{tabular}


TABLE $5-$ Continued

\begin{tabular}{|c|c|c|c|c|c|c|}
\hline SFRS & Name & $\begin{array}{l}\text { Position }^{\mathrm{a}} \\
\text { (J2000) }\end{array}$ & $\begin{array}{l}\text { Offset }^{\mathrm{b}} \\
(\operatorname{arcsec})\end{array}$ & $\underset{\substack{\mathrm{F}(1.4 \mathrm{GHz}) \\
(\mathrm{mJy})}}{\mathrm{F}}$ & References $^{\mathrm{c}}$ & Remarks $^{\mathrm{d}}$ \\
\hline 148 & NGC 3822 & $11: 42: 11.12+10: 16: 39.0$ & 1.2 & $34.0 \pm 1.1$ & 1 & \\
\hline 149 & UGC 6665 & $11: 42: 12.34+00: 20: 03.2$ & 2.1 & $32.6 \pm 1.4$ & 1 & \\
\hline 150 & MCG 3-30-051 & $11: 42: 24.49+20: 07: 11.8$ & 2.3 & $12.2 \pm 0.6$ & 1 & \\
\hline 151 & NGC 3839 & $11: 43: 54.33+10: 47: 04.7$ & 0.1 & $47.4 \pm 1.8$ & 1 & \\
\hline 152 & UGC 6732 & $11: 45: 32.92+58: 58: 42.1$ & 2.0 & $5.1 \pm 0.5$ & 1 & \\
\hline 153 & IC 730 & $11: 45: 35.24+03: 13: 54.6$ & 0.2 & $22.8 \pm 0.8$ & 1 & \\
\hline 154 & IC 732 & $11: 45: 59.83+20: 26: 28.7$ & 21.4 & $18.8 \pm 1.0$ & 1 & $\mathrm{~B}^{*}$ \\
\hline 155 & NGC 3912 & $11: 50: 04.40+26: 28: 44.7$ & 1.0 & $21.3 \pm 1.1$ & 1 & \\
\hline 156 & NGC 3928 & $11: 51: 47.44+48: 41: 01.3$ & 2.7 & $12.2 \pm 0.6$ & 1 & \\
\hline 157 & NGC 3934 & $11: 52: 12.70+16: 51: 01.8$ & 4.9 & $28.0 \pm 1.3$ & 1 & \\
\hline 158 & UGC 6865 & $11: 53: 40.08+43: 27: 37.3$ & 2.4 & $19.4 \pm 1.1$ & 1 & \\
\hline 159 & UGC 6901 & $11: 55: 38.36+43: 02: 44.4$ & 0.7 & $16.9 \pm 0.7$ & 1 & \\
\hline 160 & CGCG 013-010 & $11: 57: 06.01+01: 07: 32.1$ & 1.2 & $39.0 \pm 1.2$ & 1 & \\
\hline 161 & NGC 3991 & $11: 57: 31.08+32: 20: 16.0$ & 3.1 & $34.4 \pm 1.5$ & 1 & \\
\hline 162 & NGC 4004 & $11: 58: 05.10+27: 52: 41.7$ & 2.8 & $29.2 \pm 1.6$ & 1 & \\
\hline 163 & NGC 4014 & $11: 58: 35.82+16: 10: 38.3$ & 0.3 & $20.0 \pm 1.0$ & 1 & \\
\hline 164 & NGC 4010 & $11: 58: 36.54+47: 15: 33.0$ & 16.1 & $20.3 \pm 2.3$ & 1 & $\mathrm{E}^{*}$ \\
\hline 165 & NGC 4018 & $11: 58: 40.51+25: 19: 02.3$ & 5.0 & $29.9 \pm 1.3$ & 1 & \\
\hline 166 & NGC 4020 & $11: 58: 58.26+30: 24: 55.2$ & 23.8 & $6.8 \pm 1.7$ & 1 & $\mathrm{~B}, \mathrm{E}^{*}$ \\
\hline 167 & IRAS $11571+3003$ & $11: 59: 42.72+29: 47: 17.6$ & $\ldots$ & $1.4 \pm 0.2$ & 3 & $*$ \\
\hline 168 & UGC 7017 & $12: 02: 22.37+29: 51: 43.0$ & 2.0 & $35.6 \pm 1.5$ & 1 & \\
\hline 169 & UGC 7016 & $12: 02: 24.11+14: 50: 36.5$ & 2.0 & $11.9 \pm 0.6$ & 1 & \\
\hline 170 & MCG 3-31-030 & $12: 03: 35.71+16: 03: 15.6$ & 5.6 & $2.7 \pm 0.5$ & 1 & \\
\hline 171 & NGC 4062 & $12: 04: 02.42+31: 53: 41.6$ & 18.4 & $7.3 \pm 1.5$ & 1 & $\mathrm{E}^{*}$ \\
\hline 172 & NGC 4064 & $12: 04: 10.95+18: 26: 40.6$ & 3.3 & $9.5 \pm 0.5$ & 1 & \\
\hline 173 & CGCG 098-059 & $12: 07: 09.52+16: 59: 42.2$ & 2.0 & $18.3 \pm 1.0$ & 1 & \\
\hline 174 & NGC 4116 & $12: 07: 36.88+02: 41: 30.4$ & 2.1 & $17.3 \pm 2.7$ & 1 & \\
\hline 175 & NGC 4136 & $12: 09: 17.71+29: 55: 39.4$ & $\ldots$ & $14.6 \pm 1.7$ & 2 & $\mathrm{E}$ \\
\hline 176 & NGC 4150 & $12: 10: 33.71+30: 24: 55.2$ & $\ldots$ & $<2.0$ & 2 & $\mathrm{~B}$ \\
\hline 177 & IRAS $12086+1441$ & $12: 11: 14.43+14: 24: 41.6$ & 6.4 & $6.3 \pm 0.4$ & 1 & \\
\hline 178 & NGC 4162 & $12: 11: 53.50+24: 07: 09.7$ & 6.9 & $34.2 \pm 1.9$ & 1 & \\
\hline 179 & NGC 4178 & $12: 12: 46.13+10: 52: 06.2$ & 11.8 & $30.3 \pm 2.7$ & 4 & $\mathrm{E}^{*}$ \\
\hline 180 & IRAS $12112+0305$ & $12: 13: 45.94+02: 48: 40.8$ & 2.2 & $23.3 \pm 0.8$ & 1 & \\
\hline 181 & NGC 4189 & $12: 13: 48.85+13: 25: 30.2$ & 23.2 & $17.8 \pm 2.0$ & 4 & $\mathrm{E}^{*}$ \\
\hline 182 & NGC 4194 & $12: 14: 09.67+54: 31: 35.9$ & 0.2 & $100.7 \pm 3.0$ & 1 & \\
\hline 183 & NGC 4204 & $12: 15: 14.45+20: 39: 30.9$ & $\ldots$ & $<0.4$ & 3 & \\
\hline 184 & NGC 4207 & $12: 15: 30.42+09: 35: 05.4$ & 1.3 & $18.7 \pm 1.4$ & 1 & \\
\hline 185 & UGC 7286 & $12: 15: 59.03+27: 26: 35.1$ & 4.3 & $2.9 \pm 0.4$ & 1 & \\
\hline 186 & NGC 4234 & $12: 17: 08.89+03: 40: 55.2$ & 5.7 & $4.9 \pm 0.4$ & 1 & $\mathrm{E}^{*}$ \\
\hline 187 & NGC 4237 & $12: 17: 11.51+15: 19: 21.4$ & 5.1 & $6.0 \pm 0.5$ & 1 & \\
\hline 188 & NGC 4244 & $12: 17: 27.57+37: 47: 45.6$ & $\ldots$ & $27.7 \pm 2.8$ & 2 & $\mathrm{E}^{*}$ \\
\hline 189 & NGC 4253 & $12: 18: 26.43+29: 48: 47.3$ & 1.4 & $38.1 \pm 1.2$ & 1 & \\
\hline 190 & MCG 3-32-005 & $12: 20: 47.22+17: 00: 57.4$ & 0.5 & $25.4 \pm 0.9$ & 1 & \\
\hline 191 & NGC 4290 & $12: 20: 47.33+58: 05: 34.0$ & 1.8 & $18.9 \pm 1.3$ & 1 & \\
\hline 192 & NGC 4294 & $12: 21: 17.68+11: 30: 41.0$ & 14.1 & $21.1 \pm 1.6$ & 1 & $\mathrm{E}^{*}$ \\
\hline 193 & NGC 4314 & $12: 22: 31.94+29: 53: 45.2$ & 1.7 & $13.1 \pm 1.0$ & 1 & \\
\hline 194 & NGC 4385 & $12: 25: 42.83+00: 34: 23.4$ & 2.1 & $13.1 \pm 0.6$ & 1 & \\
\hline 195 & NGC 4395 & $12: 25: 48.86+33: 32: 48.7$ & $\cdots$ & $78.0 \pm 8.0$ & 2 & $\mathrm{E}^{*}$ \\
\hline 196 & NGC 4396 & $12: 25: 58.94+15: 40: 12.7$ & 4.3 & $20.3 \pm 2.1$ & 1 & \\
\hline 197 & NGC 4412 & $12: 26: 36.09+03: 57: 52.8$ & 0.3 & $14.6 \pm 0.6$ & 1 & \\
\hline 198 & NGC 4418 & $12: 26: 54.54-00: 52: 38.8$ & 1.4 & $40.8 \pm 1.3$ & 1 & \\
\hline 199 & NGC 4420 & $12: 26: 58.50+02: 29: 40.0$ & $\ldots$ & $<4.0$ & 2,3 & * \\
\hline 200 & NGC 4424 & $12: 27: 10.53+09: 25: 02.8$ & 19.8 & $3.9 \pm 0.6$ & 1 & $*$ \\
\hline 201 & NGC 4435 & $12: 27: 40.46+13: 04: 44.4$ & $\cdots$ & $<1.5$ & 2 & \\
\hline 202 & NGC 4438 & $12: 27: 45.56+13: 00: 31.0$ & 1.2 & $63.3 \pm 2.7$ & 1 & \\
\hline 203 & NGC 4448 & $12: 28: 15.46+28: 37: 13.1$ & $\ldots$ & $5.1 \pm 0.8$ & 2 & $\mathrm{E}^{*}$ \\
\hline 204 & $3 \mathrm{C} 273$ & $12: 29: 06.41+02: 03: 05.1$ & 5.5 & $54991.2 \pm 1900.3$ & 1 & \\
\hline 205 & NGC 4470 & $12: 29: 38.01+07: 49: 18.4$ & 9.4 & $15.5 \pm 1.6$ & 1 & \\
\hline 206 & IRAS $12274+0018$ & $12: 29: 58.80+00: 01: 38.0$ & $\ldots$ & $<0.9$ & 3 & $*$ \\
\hline 207 & NGC 4491 & $12: 30: 57.10+11: 29: 01.0$ & $\ldots$ & $<4.5$ & 3 & $*$ \\
\hline 208 & NGC 4500 & $12: 31: 22.14+57: 57: 52.9$ & 0.5 & $28.2 \pm 1.2$ & 4 & \\
\hline 209 & NGC 4495 & $12: 31: 22.82+29: 08: 11.4$ & 0.9 & $30.1 \pm 1.0$ & 1 & \\
\hline 210 & IC 3476 & $12: 32: 41.64+14: 03: 03.1$ & 3.8 & $7.5 \pm 1.1$ & 1 & \\
\hline 211 & NGC 4509 & $12: 33: 06.21+32: 05: 27.0$ & 9.9 & $2.9 \pm 0.4$ & 1 & \\
\hline 212 & NGC 4519 & $12: 33: 30.62+08: 39: 11.2$ & $\ldots$ & $25.6 \pm 1.6$ & 2 & \\
\hline 213 & NGC 4548 & $12: 35: 26.45+14: 29: 46.8$ & $\ldots$ & $5.0 \pm 2.0$ & 2 & $\mathrm{E}$ \\
\hline 214 & IRAS $12337+5044$ & $12: 36: 06.96+50: 28: 16.8$ & 3.1 & $13.0 \pm 0.5$ & 1 & \\
\hline 215 & IC 3581 & $12: 36: 37.93+24: 25: 44.1$ & 1.9 & $13.9 \pm 0.6$ & 1 & \\
\hline 216 & NGC 4592 & 12:39:18.68 -00:31:53.1 & 2.1 & $6.7 \pm 1.4$ & 1 & \\
\hline 217 & NGC 4607 & $12: 41: 12.75+11: 53: 12.1$ & 7.8 & $22.9 \pm 1.6$ & 1 & \\
\hline 218 & NGC 4625 & $12: 41: 52.73+41: 16: 26.2$ & $\ldots$ & $5.6 \pm 2.0$ & 2 & $\mathrm{D}, \mathrm{E}^{*}$ \\
\hline 219 & NGC 4630 & $12: 42: 31.98+03: 57: 39.6$ & 13.1 & $12.6 \pm 1.2$ & 1 & $\mathrm{E}^{*}$ \\
\hline 220 & IC 3690 & $12: 42: 49.29+10: 21: 22.3$ & 4.8 & $6.9 \pm 0.5$ & 1 & \\
\hline 221 & UGC 7905 & $12: 43: 47.88+54: 53: 48.0$ & 2.8 & $16.9 \pm 0.6$ & 1 & \\
\hline
\end{tabular}


TABLE $5-$ Continued

\begin{tabular}{|c|c|c|c|c|c|c|}
\hline SFRS & Name & $\begin{array}{l}\text { Position }^{\mathrm{a}} \\
(\mathrm{J} 2000)\end{array}$ & $\begin{array}{l}\text { Offset }^{\mathrm{b}} \\
(\operatorname{arcsec})\end{array}$ & $\begin{array}{c}\mathrm{F}(1.4 \mathrm{GHz}) \\
(\mathrm{mJy})\end{array}$ & References $^{\mathrm{c}}$ & Remarks $^{\mathrm{d}}$ \\
\hline 222 & MCG 5-30-069 & $12: 44: 41.27+26: 25: 10.7$ & 0.3 & $9.8 \pm 0.5$ & 1 & \\
\hline 223 & IC 3721 & $12: 44: 53.16+18: 45: 18.4$ & 0.9 & $23.5 \pm 1.1$ & 1 & \\
\hline 224 & NGC 4670 & $12: 45: 17.11+27: 07: 30.4$ & 2.6 & $13.7 \pm 1.0$ & 1 & \\
\hline 225 & NGC 4675 & $12: 45: 32.02+54: 44: 15.4$ & 1.1 & $14.2 \pm 0.6$ & 1 & \\
\hline 226 & MCG 7-26-051 & $12: 46: 56.70+42: 16: 00.4$ & 2.0 & $31.3 \pm 1.0$ & 1 & \\
\hline 227 & NGC 4689 & $12: 47: 44.61+13: 45: 44.1$ & 13.9 & $12.5 \pm 1.8$ & 1 & $\mathrm{E}$ \\
\hline 228 & NGC 4688 & $12: 47: 44.35+04: 19: 04.4$ & $\ldots$ & $<2.6$ & 2 & $*$ \\
\hline 229 & NGC 4704 & $12: 48: 46.57+41: 55: 17.7$ & 1.9 & $9.8 \pm 0.9$ & 1 & \\
\hline 230 & NGC 4701 & $12: 49: 11.68+03: 23: 21.5$ & 2.5 & $18.5 \pm 1.6$ & 1 & \\
\hline 231 & IRAS $12468+3436$ & $12: 49: 17.27+34: 19: 52.1$ & $\cdots$ & $5.1 \pm 0.2$ & 1,3 & * \\
\hline 232 & IRAS $12470+1404$ & $12: 49: 34.78+13: 48: 11.4$ & $\ldots$ & $2.3 \pm 0.2$ & 1,3 & * \\
\hline 233 & MCG 8-23-097 & $12: 50: 39.80+47: 56: 00.6$ & 0.5 & $29.7 \pm 1.0$ & 1 & \\
\hline 234 & NGC 4747 & $12: 51: 45.59+25: 46: 26.1$ & 4.0 & $7.4 \pm 0.5$ & 1 & \\
\hline 235 & UGC 8017 & $12: 52: 53.59+28: 22: 16.1$ & 0.5 & $28.9 \pm 1.2$ & 1 & \\
\hline 236 & NGC 4765 & $12: 53: 14.43+04: 27: 50.8$ & 3.7 & $17.6 \pm 1.3$ & 1 & \\
\hline 237 & VCC 2096 & $12: 53: 24.79+11: 42: 38.1$ & 1.7 & $19.9 \pm 0.7$ & 1 & \\
\hline 238 & UGC 8041 & $12: 55: 12.60+00: 07: 00.0$ & $\ldots$ & $<1.5$ & 3 & $\mathrm{E}$ \\
\hline 239 & UGC 8058 & $12: 56: 14.13+56: 52: 23.8$ & 1.8 & $308.9 \pm 2.1$ & 1 & \\
\hline 240 & NGC 4837 & $12: 56: 48.30+48: 17: 50.7$ & 1.7 & $25.5 \pm 1.1$ & 1 & \\
\hline 241 & UM 530 & $12: 58: 08.45+01: 51: 43.1$ & $\ldots$ & $4.3 \pm 0.5$ & 3 & $\mathrm{~A}^{*}$ \\
\hline 242 & NGC 4861 & $12: 59: 00.25+34: 50: 48.3$ & $\ldots$ & $14.4 \pm 1.4$ & 2 & $\mathrm{D}, \mathrm{E}^{*}$ \\
\hline 243 & NGC 4868 & $12: 59: 08.92+37: 18: 36.8$ & 0.7 & $26.5 \pm 1.4$ & 1 & \\
\hline 244 & NGC 4922 & $13: 01: 25.23+29: 18: 50.3$ & 1.0 & $38.8 \pm 1.2$ & 1 & \\
\hline 245 & UGC 8179 & $13: 05: 14.13+31: 59: 58.0$ & 1.1 & $16.6 \pm 1.0$ & 1 & \\
\hline 246 & NGC 5001 & $13: 09: 33.47+53: 29: 39.2$ & 3.1 & $24.6 \pm 1.1$ & 1 & \\
\hline 247 & IC 856 & $13: 10: 41.67+20: 32: 13.5$ & 5.6 & $12.6 \pm 0.6$ & 1 & \\
\hline 248 & UGC 8269 & $13: 11: 15.11+46: 42: 00.6$ & 1.8 & $18.7 \pm 0.7$ & 1 & \\
\hline 249 & NGC 5014 & $13: 11: 31.16+36: 16: 55.1$ & 1.0 & $11.4 \pm 0.5$ & 1 & \\
\hline 250 & NGC 5012 & $13: 11: 37.05+22: 54: 53.0$ & 2.8 & $29.7 \pm 1.9$ & 1 & \\
\hline 251 & IRAS $13116+4508$ & $13: 13: 47.90+44: 52: 59.0$ & & $<0.5$ & 3 & \\
\hline 252 & IC 860 & $13: 15: 03.47+24: 37: 07.2$ & 0.7 & $30.8 \pm 1.0$ & 1 & \\
\hline 253 & IRAS $13144+4508$ & $13: 16: 39.64+44: 52: 35.1$ & 1.2 & $5.9 \pm 0.4$ & 1 & \\
\hline 254 & NGC 5060 & $13: 17: 16.29+06: 02: 14.0$ & 1.2 & $16.2 \pm 0.6$ & 1 & \\
\hline 255 & UGC 8357 & $13: 17: 58.74-00: 18: 42.5$ & 1.0 & $20.4 \pm 1.0$ & 1 & \\
\hline 256 & UGC 8361 & $13: 18: 18.60+06: 20: 06.4$ & 1.0 & $17.3 \pm 0.7$ & 1 & \\
\hline 257 & IC 883 & $13: 20: 35.38+34: 08: 22.6$ & 1.1 & $104.4 \pm 3.2$ & 1 & \\
\hline 258 & NGC 5100 & $13: 20: 59.58+08: 58: 41.5$ & 0.5 & $102.3 \pm 3.1$ & 1 & \\
\hline 259 & NGC 5104 & $13: 21: 23.16+00: 20: 33.4$ & 1.3 & $39.9 \pm 1.3$ & 1 & \\
\hline 260 & NGC 5107 & $13: 21: 23.40+38: 32: 18.5$ & 15.5 & $4.5 \pm 0.5$ & 1 & * \\
\hline 261 & NGC 5112 & $13: 21: 59.25+38: 44: 17.0$ & $\ldots$ & $22.4 \pm 3.0$ & 2 & $\mathrm{E}^{*}$ \\
\hline 262 & NGC 5123 & $13: 23: 10.45+43: 05: 13.0$ & 2.6 & $18.7 \pm 1.3$ & 1 & \\
\hline 263 & IRAS $13218+0552$ & $13: 24: 19.96+05: 37: 01.2$ & $\ldots$ & $2.8 \pm 0.2$ & 3 & * \\
\hline 264 & IRAS $13232+1731$ & $13: 25: 43.79+17: 15: 53.2$ & 1.3 & $11.2 \pm 0.5$ & 1 & \\
\hline 265 & NGC 5147 & $13: 26: 20.29+02: 06: 02.9$ & 8.4 & $25.2 \pm 2.6$ & 1 & \\
\hline 266 & NGC 5204 & $13: 29: 36.38+58: 24: 46.2$ & $\cdots$ & $18.8 \pm 4.4$ & 2 & $\mathrm{E}$ \\
\hline 267 & UGC 8502 & $13: 30: 38.79+31: 17: 06.7$ & 8.3 & $7.7 \pm 0.5$ & 1 & \\
\hline 268 & UGC 8561 & $13: 34: 57.16+34: 02: 38.9$ & 1.3 & $29.3 \pm 1.5$ & 1 & \\
\hline 269 & NGC 5230 & $13: 35: 31.05+13: 40: 33.4$ & 12.0 & $14.5 \pm 1.7$ & 1 & * \\
\hline 270 & IRAS $13349+2438$ & $13: 37: 18.73+24: 23: 02.8$ & 0.6 & $19.6 \pm 0.7$ & 1 & \\
\hline 271 & NGC 5256 & $13: 38: 17.60+48: 16: 38.4$ & 5.3 & $126.3 \pm 4.5$ & 1 & \\
\hline 272 & UGC 8626 & $13: 38: 23.52+06: 53: 14.7$ & 1.2 & $6.3 \pm 0.4$ & 1 & \\
\hline 273 & NGC 5263 & $13: 39: 55.53+28: 23: 59.4$ & 3.7 & $38.0 \pm 1.8$ & 1 & \\
\hline 274 & MCG 1-35-028 & $13: 40: 27.31+04: 46: 27.8$ & 2.7 & $15.8 \pm 0.6$ & 1 & \\
\hline 275 & IC 910 & $13: 41: 07.85+23: 16: 56.2$ & 0.8 & $38.7 \pm 1.2$ & 1 & \\
\hline 276 & MK 268 & $13: 41: 11.09+30: 22: 41.3$ & 0.6 & $42.5 \pm 1.3$ & 1 & \\
\hline 277 & NGC 5278 & $13: 41: 39.81+55: 40: 14.7$ & 4.8 & $23.1 \pm 1.1$ & 1 & \\
\hline 278 & NGC 5273 & $13: 42: 08.92+35: 39: 12.4$ & 7.3 & $3.5 \pm 0.4$ & 1 & \\
\hline 279 & UGC 8685 & $13: 43: 08.76+30: 20: 14.7$ & 1.5 & $15.4 \pm 0.6$ & 1 & \\
\hline 280 & UGC 8686 & $13: 43: 40.34+03: 53: 48.5$ & 3.4 & $14.7 \pm 0.6$ & 1 & \\
\hline 281 & UGC 8696 & $13: 44: 42.21+55: 53: 13.1$ & 0.6 & $158.8 \pm 6.9$ & 2,4 & $\mathrm{E}$ \\
\hline 282 & NGC 5297 & $13: 46: 23.79+43: 52: 08.7$ & 11.8 & $23.0 \pm 2.4$ & 1 & $\mathrm{E}^{*}$ \\
\hline 283 & MK 796 & $13: 46: 49.47+14: 24: 03.1$ & 1.5 & $190.6 \pm 6.7$ & 1 & \\
\hline 284 & IRAS $13446+1121$ & $13: 47: 04.43+11: 06: 22.9$ & 0.9 & $20.4 \pm 0.7$ & 1 & \\
\hline 285 & NGC 5303 & $13: 47: 45.07+38: 18: 19.6$ & 3.0 & $24.9 \pm 1.5$ & 1 & \\
\hline 286 & NGC 5313 & $13: 49: 44.15+39: 59: 05.7$ & 2.3 & $44.3 \pm 2.0$ & 1 & \\
\hline 287 & MCG 3-35-034 & $13: 53: 09.52+14: 39: 22.2$ & 2.5 & $20.8 \pm 0.7$ & 1 & \\
\hline 288 & NGC 5347 & $13: 53: 18.04+33: 29: 25.6$ & 3.6 & $5.6 \pm 0.5$ & 1 & \\
\hline 289 & NGC 5350 & $13: 53: 21.28+40: 21: 48.5$ & 4.3 & $16.8 \pm 1.7$ & 1 & \\
\hline 290 & NGC 5368 & $13: 54: 29.31+54: 19: 50.3$ & 1.3 & $9.1 \pm 0.5$ & 1 & \\
\hline 291 & UGC 8827 & $13: 54: 31.24+15: 02: 39.1$ & 1.0 & $23.0 \pm 1.1$ & 1 & \\
\hline 292 & UGC 8850 & $13: 56: 02.86+18: 22: 19.0$ & 3.6 & $380.5 \pm 1.4$ & 1 & \\
\hline 293 & UGC 8856 & $13: 56: 07.83+30: 04: 52.3$ & 1.0 & $21.7 \pm 0.8$ & 1 & \\
\hline 294 & NGC 5374 & $13: 57: 29.73+06: 05: 49.7$ & 1.5 & $24.2 \pm 1.4$ & 1 & \\
\hline 295 & UGC 8902 & $13: 59: 02.80+15: 33: 54.7$ & 1.9 & $12.4 \pm 1.0$ & 1 & \\
\hline
\end{tabular}


TABLE $5-$ Continued

\begin{tabular}{|c|c|c|c|c|c|c|}
\hline SFRS & Name & $\begin{array}{l}\text { Position }^{\mathrm{a}} \\
\text { (J2000) }\end{array}$ & $\begin{array}{l}\text { Offset }^{\mathrm{b}} \\
\text { (arcsec) }\end{array}$ & $\begin{array}{c}\mathrm{F}(1.4 \mathrm{GHz}) \\
(\mathrm{mJy})\end{array}$ & References $^{\mathrm{c}}$ & Remarks $^{\mathrm{d}}$ \\
\hline 296 & NGC 5403 & $13: 59: 51.04+38: 10: 56.3$ & 3.3 & $37.4 \pm 1.8$ & 1 & \\
\hline 297 & MCG 7-29-036 & $14: 00: 57.84+42: 51: 20.4$ & $\ldots$ & $5.2 \pm 0.6$ & 2 & $\mathrm{D}^{*}$ \\
\hline 298 & NGC 5414 & $14: 02: 03.42+09: 55: 44.2$ & 2.1 & $19.6 \pm 1.3$ & 1 & \\
\hline 299 & MCG 5-33-046 & $14: 04: 47.88+30: 44: 36.2$ & 1.8 & $10.4 \pm 0.5$ & 1 & \\
\hline 300 & NGC 5474 & $14: 05: 01.42+53: 39: 44.4$ & $\ldots$ & $13.0 \pm 3.0$ & 2 & $\mathrm{E}^{*}$ \\
\hline 301 & NGC 5480 & $14: 06: 21.86+50: 43: 31.0$ & 2.8 & $29.0 \pm 1.5$ & 1 & \\
\hline 302 & MCG 6-31-070 & $14: 06: 48.99+33: 46: 16.2$ & 2.4 & $18.1 \pm 0.7$ & 1 & \\
\hline 303 & CGCG 074-129 & $14: 10: 41.42+13: 33: 26.1$ & 2.9 & $7.6 \pm 0.5$ & 1 & \\
\hline 304 & NGC 5520 & $14: 12: 22.87+50: 20: 54.2$ & 0.7 & $14.8 \pm 0.6$ & 1 & \\
\hline 305 & NGC 5515 & $14: 12: 38.17+39: 18: 36.3$ & 0.4 & $28.8 \pm 1.0$ & 1 & \\
\hline 306 & NGC 5526 & $14: 13: 53.78+57: 46: 17.5$ & 0.7 & $15.9 \pm 0.9$ & 1 & \\
\hline 307 & NGC 5522 & $14: 14: 50.33+15: 08: 52.6$ & 3.9 & $17.6 \pm 1.0$ & 1 & \\
\hline 308 & NGC 5541 & $14: 16: 31.88+39: 35: 23.3$ & 2.9 & $33.0 \pm 1.4$ & 1 & \\
\hline 309 & IC 4395 & $14: 17: 21.09+26: 51: 28.4$ & 1.6 & $22.6 \pm 0.8$ & 1 & \\
\hline 310 & UGC 9165 & $14: 18: 47.66+24: 56: 24.9$ & 2.0 & $30.6 \pm 1.3$ & 1 & \\
\hline 311 & MK 1490 & $14: 19: 43.28+49: 14: 12.3$ & 0.7 & $20.2 \pm 0.7$ & 1 & \\
\hline 312 & NGC 5585 & $14: 19: 48.19+56: 43: 45.6$ & $\cdots$ & $18.7 \pm 2.9$ & 2 & $\mathrm{E}^{*}$ \\
\hline 313 & IC 4408 & $14: 21: 12.96+29: 59: 36.2$ & 2.0 & $20.1 \pm 0.7$ & 1 & \\
\hline 314 & NGC 5584 & $14: 22: 25.36-00: 23: 31.2$ & $\ldots$ & $19.4 \pm 2.1$ & 4 & $\mathrm{E}$ \\
\hline 315 & NGC 5633 & $14: 27: 28.39+46: 08: 49.3$ & 1.7 & $27.9 \pm 1.5$ & 1 & \\
\hline 316 & NGC 5660 & $14: 29: 49.79+49: 37: 25.5$ & 3.9 & $33.4 \pm 2.1$ & 1 & \\
\hline 317 & NGC 5656 & $14: 30: 25.35+35: 19: 16.2$ & 2.1 & $20.8 \pm 1.3$ & 1 & \\
\hline 318 & NGC 5657 & $14: 30: 43.68+29: 10: 47.5$ & 7.3 & $10.3 \pm 0.9$ & 1,2 & \\
\hline 319 & CGCG 133-083 & $14: 31: 54.03+21: 56: 20.2$ & 2.1 & $11.1 \pm 0.5$ & 1 & * \\
\hline 320 & MCG 7-30-028 & $14: 33: 48.36+40: 05: 38.9$ & $\ldots$ & $12.6 \pm 0.5$ & 2 & $\mathrm{D}$ \\
\hline 321 & MCG 6-32-070 & $14: 35: 18.28+35: 07: 08.5$ & 1.8 & $22.0 \pm 0.8$ & 1 & \\
\hline 322 & UGC 9412 & $14: 36: 22.07+58: 47: 41.6$ & 2.3 & $11.2 \pm 0.5$ & 1 & \\
\hline 323 & NGC 5698 & $14: 37: 14.47+38: 27: 18.1$ & 3.8 & $4.9 \pm 0.5$ & 1 & \\
\hline 324 & NGC 5691 & $14: 37: 53.36-00: 23: 54.2$ & 1.8 & $19.0 \pm 1.3$ & 1 & \\
\hline 325 & MCG 9-24-035 & $14: 45: 45.04+51: 34: 50.0$ & 1.2 & $11.5 \pm 0.5$ & 1 & \\
\hline 326 & MCG 9-24-038 & $14: 46: 37.29+56: 14: 00.8$ & 2.8 & $13.8 \pm 0.6$ & 1 & \\
\hline 327 & UGC 9560 & $14: 50: 56.18+35: 34: 13.9$ & 7.4 & $5.9 \pm 0.5$ & 1 & \\
\hline 328 & IC 1076 & $14: 54: 59.56+18: 02: 14.3$ & 0.8 & $20.7 \pm 0.7$ & 1 & \\
\hline 329 & IRAS $14538+1730$ & $14: 56: 08.47+17: 18: 35.4$ & 1.4 & $12.2 \pm 0.5$ & 1 & * \\
\hline 330 & NGC 5795 & $14: 56: 19.35+49: 23: 56.1$ & 0.7 & $18.3 \pm 1.0$ & 1,2 & $\mathrm{E}$ \\
\hline 331 & UGC 9618 & $14: 57: 00.69+24: 37: 01.8$ & 1.4 & $93.1 \pm 3.3$ & 1 & \\
\hline 332 & UGC 9639 & $14: 58: 36.00+44: 53: 02.2$ & 1.1 & $35.7 \pm 1.1$ & 1 & \\
\hline 333 & MCG 6-33-022 & $15: 08: 05.64+34: 23: 23.2$ & 5.8 & $133.1 \pm 4.0$ & 1 & \\
\hline 334 & NGC 5879 & $15: 09: 46.73+56: 59: 56.5$ & 4.2 & $19.5 \pm 1.6$ & 1 & \\
\hline 335 & MCG 9-25-036 & $15: 12: 52.33+51: 23: 55.0$ & .. & $5.0 \pm 0.5$ & 2 & $\mathrm{D}^{*}$ \\
\hline 336 & NGC 5899 & $15: 15: 03.60+42: 03: 05.1$ & 7.0 & $41.9 \pm 2.0$ & 1 & \\
\hline 337 & NGC 5905 & $15: 15: 23.30+55: 31: 00.6$ & 1.7 & $20.1 \pm 1.5$ & 1 & \\
\hline 338 & MK 848 & $15: 18: 06.20+42: 44: 42.5$ & 2.6 & $50.3 \pm 1.6$ & 1 & \\
\hline 339 & IC 4553 & $15: 34: 57.26+23: 30: 11.1$ & 2.2 & $326.3 \pm 9.8$ & 1 & \\
\hline 340 & UGC 9922 & $15: 35: 54.00+38: 40: 29.8$ & 2.4 & $22.0 \pm 1.0$ & 1 & \\
\hline 341 & IC 4567 & $15: 37: 13.42+43: 17: 55.9$ & 2.5 & $23.9 \pm 1.3$ & 1 & \\
\hline 342 & MCG 4-37-016 & $15: 39: 26.77+24: 56: 45.6$ & 11.6 & $20.1 \pm 1.0$ & 1 & $\mathrm{~B}^{*}$ \\
\hline 343 & NGC 5975 & $15: 39: 57.99+21: 28: 13.2$ & 1.2 & $22.1 \pm 0.8$ & 1 & \\
\hline 344 & NGC 5980 & $15: 41: 30.44+15: 47: 16.5$ & 0.9 & $25.3 \pm 1.2$ & 1 & \\
\hline 345 & NGC 5992 & $15: 44: 21.63+41: 05: 10.3$ & 1.5 & $16.0 \pm 0.6$ & 1 & \\
\hline 346 & NGC 5996 & $15: 46: 58.87+17: 53: 04.1$ & 1.0 & $29.6 \pm 1.6$ & 1 & \\
\hline 347 & IRAS $15519+3537$ & $15: 53: 48.80+35: 28: 10.4$ & 8.2 & $11.4 \pm 0.8$ & 1 & \\
\hline 348 & UGC 10099 & $15: 56: 36.40+41: 52: 50.6$ & 0.1 & $14.8 \pm 0.6$ & 1 & \\
\hline 349 & MCG 5-38-006 & $15: 58: 43.54+26: 49: 04.1$ & 2.5 & $10.8 \pm 0.5$ & 1 & \\
\hline 350 & UGC 10120 & $15: 59: 10.07+35: 01: 45.6$ & 5.8 & $5.5 \pm 0.4$ & 1 & \\
\hline 351 & NGC 6027A & $15: 59: 11.18+20: 45: 16.8$ & $\ldots$ & $11.3 \pm 1.0$ & 2 & $\mathrm{E}^{*}$ \\
\hline 352 & NGC 6040 & $16: 04: 26.52+17: 44: 33.3$ & 2.1 & $95.9 \pm 2.9$ & 1 & \\
\hline 353 & UGC 10200 & $16: 05: 45.61+41: 20: 43.8$ & 4.2 & $11.2 \pm 0.9$ & 1 & \\
\hline 354 & IRAS $16052+5334$ & $16: 06: 32.84+53: 26: 32.3$ & 1.4 & $10.2 \pm 0.5$ & 1 & \\
\hline 355 & IRAS $16053+1836$ & $16: 07: 38.39+18: 28: 46.9$ & 2.3 & $14.0 \pm 0.6$ & 1 & \\
\hline 356 & NGC 6090 & $16: 11: 40.75+52: 27: 26.5$ & 5.3 & $48.0 \pm 1.5$ & 1 & \\
\hline 357 & UGC 10273 & $16: 12: 44.93+28: 17: 11.5$ & 3.6 & $22.2 \pm 1.1$ & 1 & \\
\hline 358 & IRAS $16150+2233$ & $16: 17: 08.79+22: 26: 28.4$ & $\ldots$ & $3.9 \pm 0.3$ & 1.3 & * \\
\hline 359 & UGC 10322 & $16: 18: 08.10+22: 13: 32.0$ & 3.5 & $24.4 \pm 1.1$ & 1 & \\
\hline 360 & NGC 6120 & $16: 19: 48.16+37: 46: 28.6$ & 1.0 & $33.7 \pm 1.1$ & 1 & \\
\hline 361 & MCG 3-42-004 & $16: 24: 15.13+20: 10: 59.0$ & 1.8 & $10.3 \pm 0.5$ & 1 & \\
\hline 362 & UGC 10407 & $16: 28: 28.19+41: 13: 04.6$ & 3.7 & $18.4 \pm 0.7$ & 1 & \\
\hline 363 & IRAS $16320+3922$ & $16: 33: 49.62+39: 15: 49.0$ & $\ldots$ & $6.5 \pm 0.5$ & 1,3 & * \\
\hline 364 & NGC 6186 & $16: 34: 25.49+21: 32: 27.2$ & $\ldots$ & $17.4 \pm 1.1$ & 2 & $\mathrm{D}, \mathrm{E}^{*}$ \\
\hline 365 & MCG 9-27-053 & $16: 35: 15.46+52: 46: 49.8$ & 0.4 & $20.0 \pm 0.7$ & 1 & \\
\hline 366 & UGC 10514 & $16: 42: 23.66+25: 05: 10.7$ & 0.8 & $31.9 \pm 1.3$ & 1 & \\
\hline 367 & IRAS $16435+2154$ & $16: 45: 40.71+21: 49: 18.4$ & 0.7 & $11.6 \pm 0.5$ & 1 & \\
\hline 368 & IC 4623 & $16: 51: 05.29+22: 31: 40.9$ & 2.3 & $11.0 \pm 0.5$ & 1 & \\
\hline 369 & IRAS $16516+3030$ & $16: 53: 37.34+30: 26: 09.7$ & 2.2 & $13.1 \pm 0.6$ & 1 & \\
\hline
\end{tabular}


TABLE 5 - Continued

\begin{tabular}{|c|c|c|c|c|c|c|}
\hline SFRS & Name & $\begin{array}{l}\text { Position }^{\mathrm{a}} \\
(\mathrm{J} 2000)\end{array}$ & $\begin{array}{l}\text { Offset }^{\mathrm{b}} \\
(\text { arcsec) }\end{array}$ & $\begin{array}{c}\mathrm{F}(1.4 \mathrm{GHz}) \\
(\mathrm{mJy})\end{array}$ & References $^{\mathrm{c}}$ & Remarks $^{\mathrm{d}}$ \\
\hline
\end{tabular}

Note. - Table 5 is published in its entirety in the electronic edition of Publications of the Astronomical Society of the Pacific. A portion is shown here for guidance regarding its form and content.

a Coordinates correspond to the centroids measured in the NVSS or AA319 images.

b Offset measured relative to the IRAC position.

c References. 1. NVSS query; 2. NVSS image; 3. AA319 data; 4. Condon et al. (2002).

d Remarks. $\mathrm{A}=$ Radio source double or triple; $\mathrm{B}=$ likely blended; $\mathrm{D}=$ deconvolved blend; $\mathrm{E}=$ extended source. Further details for individual sources marked with an asterisk (*): 3. high integral, $110^{\prime \prime} \times 105^{\prime \prime}$ at $30^{\circ}$ position angle $(\mathrm{PA}) ; 10$. $55^{\prime \prime} \times 43^{\prime \prime}$ at $29^{\circ} \mathrm{PA}$, excludes $4.4 \mathrm{mJy}$ point source at 08:11:16.1 +25:10:55; 12. extended with off-center bright spot, $58^{\prime \prime} \times 43^{\prime \prime}$ at $51^{\circ} \mathrm{PA} ; \mathbf{1 8}$. $58^{\prime \prime} \times 40^{\prime \prime}$ blended with IC $2338(6.9 \mathrm{mJy}) ; \mathbf{4 7}$. blended with $5.2 \mathrm{mJy}$ source at 09:15:22.4 +38:55:42; 65. blended with $z=0.22$ QSO (7.8 mJy at 09:46:03.94 +01:39:23.7), cf. 9.4 mJy for this galaxy in reference $4 ; \mathbf{7 4}$. needle galaxy in IR, $101^{\prime \prime} \times<38^{\prime \prime}$ at $-47^{\circ} \mathrm{PA} ; \mathbf{8 0}$. two point sources $16 \mathrm{mJy}$ plus $6 \mathrm{mJy}$ diffuse attributed to galaxy; 83 . radio source is star-forming region in galaxy outskirts; 98. $179^{\prime \prime} \times 111^{\prime \prime}$ at $106^{\circ} \mathrm{PA}$, flux excludes $4.4 \mathrm{mJy}$ point source at 10:42:02.5 $+13: 45: 04$; 102. $41^{\prime \prime} \times 34^{\prime \prime}$ at $153^{\circ} \mathrm{PA} ; 103$. excludes two point sources $\left(23,37 \mathrm{mJy}\right.$ at $30-50^{\prime \prime}$ separation); 106. $88^{\prime \prime} \times 46^{\prime \prime}$ at $35^{\circ} \mathrm{PA} ; \mathbf{1 0 7}$. excludes $9.4 \mathrm{mJy}$ source $1^{\prime}$ distant; 110. $183^{\prime \prime} \times 46^{\prime \prime}$ at $24^{\circ} \mathrm{PA}$, cf. $19.4 \mathrm{mJy}$ in reference 4 ; 115. excludes $22 \mathrm{mJy}$ source at 11:09:37.4 + 26:55:16, flux uncertain; 119. excludes $>4.6$ mJy, possibly extended, source to $\mathrm{S} ; \mathbf{1 2 0}$. excludes $8.9 \mathrm{mJy}$ point source at 11:16:52.4, $+59: 30: 56$; 126. extended $>60^{\prime \prime}$, position uncertainty $11^{\prime \prime} ;$ 128. galaxy compact with bright nucleus in IR, radio $53^{\prime \prime} \times 21^{\prime \prime}$ at $-4^{\circ} \mathrm{PA}$ and offset from galaxy; $\mathbf{1 3 4}$. $107^{\prime \prime} \times 47^{\prime \prime}$ at $134^{\circ} \mathrm{PA} ; 1$ 141. excludes UGC 6587 (4.0 mJy at 11:37:09.53 +02:50:20.4); 147. $57^{\prime \prime} \times 40^{\prime \prime}$ at $144^{\circ} \mathrm{PA}$, excludes $19.2 \mathrm{mJy}$ source $50^{\prime \prime}$ to $\mathrm{NW}$; 154 . two galaxies blended, IRAS flux is sum; 164. needle galaxy in reference $1,118^{\prime \prime} \times 36^{\prime \prime}$ at $62^{\circ} \mathrm{PA}$, high integral; 166 . major axis $>75^{\prime \prime}$, radio offset from IR; 167. cf. $2.8 \pm 0.5 \mathrm{mJy}$ in reference 1 ; 171. major axis $>60^{\prime \prime} ; \mathbf{1 7 9}$. $126^{\prime \prime} \times 53^{\prime \prime}$ at $43^{\circ}$ PA, radio image shows four point sources plus diffuse emission; 181. $86^{\prime \prime} \times 42^{\prime \prime}$ at $72^{\circ} \mathrm{PA}$, off-nuclear star-forming regions; 186. radio diameter $<36^{\prime \prime}$, clumpy and extended in IR; $\mathbf{1 8 8}$. needle galaxy $>11^{\prime}$, measured flux in polygon; 192. $60^{\prime \prime} \times 29^{\prime \prime}$ at $-34^{\circ} \mathrm{PA}$, clumpy in IR; 195. clumpy in IR and in radio image (reference 2 ); 199. noise affected by $3 \mathrm{C} 273$ sidelobes; 200. high integral, radio image shows linear artifacts (reference 2 ); 203. $76^{\prime \prime} \times 49^{\prime \prime}$ at $125^{\circ} \mathrm{PA} ; \mathbf{2 0 6}$. cf. $2.6 \pm 0.4$ in reference 1, noise mildly affected by $3 \mathrm{C} 273$ sidelobes; 207. noise affected by M87 sidelobes; 218. assumed Gaussian diameter 40"; 219. major axis $56^{\prime \prime}$; 228. $56^{\prime \prime} \times 56^{\prime \prime}$ high integral; 231. cf. $4.8 \pm 0.4$ in reference 1 ; 232. cf. $2.3 \pm 0.4$ in reference 1 ; 241. lobes $41,9.4$ mJy; cf. $6.4 \pm 0.6$ in reference 1; 242. elongated galaxy, radio flux includes 10.5 mJy point source (12:59:00.4, 34:50:44); 260. radio diameter <70", clumpy in IR; 261. measured in polygon, cf. $17.3 \mathrm{mJy}$ in reference $4 ; \mathbf{2 6 3}$. cf. $4.9 \pm 0.5$ in reference $1 ; 269$. $59^{\prime \prime} \times 44^{\prime \prime}$ at $71^{\circ} \mathrm{PA}$, excludes $5 \mathrm{mJy}$ point source $1^{\prime}$ to $\mathrm{NE} ; \mathbf{2 8 3}$. $107^{\prime \prime} \times 53^{\prime \prime}$ at $-45^{\circ} \mathrm{PA}$, high integral, excludes $5 \mathrm{mJy}$ point source to NW; 297. excludes $5.7 \mathrm{mJy}$ point source at $14: 00: 58.77$ $+42: 50: 42.4 ; 300$. position is brightest visible/IR clump, radio flux measured in circle based on IR image; 312 . galaxy clumpy and extended in IR, radio flux measured in circle based on IR image; 319. excludes $6.5 \mathrm{mJy}$ point source UGC 9376 at 14:33:46.83 +40:04:51.3; 329. major axis $27^{\prime \prime}$, flux includes $1 \mathrm{mJy} \mathrm{H} \mathrm{II} \mathrm{reg} \mathrm{at} \mathrm{14:56:22.42} \mathrm{+49:24:06.4;} 335$. excludes 0.8 mJy point source galaxy at 15:12:50.66 +51:23:28.9; $\mathbf{3 4 2}$. includes companion $24^{\prime \prime} \mathrm{SW}$, IRAS flux is sum; 351. $48^{\prime \prime} \times 24^{\prime \prime}$ at $17^{\circ} \mathrm{PA}$, excludes $6.7 \mathrm{mJy}$ point source at $15: 59: 10.2+20: 46: 22$; cf. $9.3 \pm 1.0 \mathrm{mJy}$ in reference 1 ; 358. cf. $3.1 \pm 0.4 \mathrm{mJy}$ in reference 1 ; 363. cf. $9.4 \pm 0.6 \mathrm{mJy}$ in reference $1 ; 364.58^{\prime \prime} \times 48^{\prime \prime}$ at $45^{\circ} \mathrm{PA}$, excludes $7.1 \mathrm{mJy}$ point source at $16: 34: 28.7+21: 32: 15$. 
TABLE 7

Far-Infrared Photometry for SFRS Galaxies

\begin{tabular}{|c|c|c|c|c|c|c|c|c|}
\hline SFRS & Name & $\begin{array}{l}\mathrm{L}(\mathrm{TIR}) \\
\left(\mathrm{L}_{\odot}\right)\end{array}$ & $\begin{array}{c}\operatorname{MIPS} \mathrm{F}(24)^{\mathrm{a}} \\
(\mathrm{Jy})\end{array}$ & $\begin{array}{c}\text { IRAS F(60) } \\
(\mathrm{Jy})\end{array}$ & $\begin{array}{c}\text { IRAS F(100) } \\
(\mathrm{Jy})\end{array}$ & $\begin{array}{c}\text { Planck F(350) } \\
(\mathrm{Jy})\end{array}$ & $\begin{array}{c}\text { Planck F(550) } \\
(\mathrm{Jy})\end{array}$ & $\begin{array}{c}\text { Planck (F850) } \\
(\mathrm{Jy})\end{array}$ \\
\hline 1 & IC 486 & 10.90 & $0.44^{\mathrm{b}}$ & 0.99 & $<1.51$ & $\ldots$ & $\ldots$ & $\ldots$ \\
\hline 2 & IC 2217 & 10.74 & $<0.32$ & 2.46 & 4.81 & $\cdots$ & $\cdots$ & $\cdots$ \\
\hline 3 & NGC 2500 & 9.34 & 0.22 & 2.81 & 5.75 & $3.02 \pm 0.14$ & $\cdots$ & $\cdots$ \\
\hline 4 & NGC 2512 & 10.88 & $0.66^{\mathrm{b}}$ & 3.80 & 7.31 & $\ldots$ & $\ldots$ & $\ldots$ \\
\hline 5 & MCG 6-18-009 & 11.25 & $<0.27$ & 1.65 & 3.09 & $\cdots$ & $\cdots$ & $\cdots$ \\
\hline 6 & MK 1212 & 11.35 & $<0.33$ & 1.87 & 3.36 & $\cdots$ & $\cdots$ & $\cdots$ \\
\hline 7 & IRAS $08072+1847$ & 10.72 & $0.74^{\mathrm{b}}$ & 2.79 & 3.08 & $\ldots$ & $\ldots$ & $\cdots$ \\
\hline 8 & NGC 2532 & 11.09 & 0.63 & 3.61 & 10.34 & $4.02 \pm 0.21$ & $1.43 \pm 0.08$ & $\cdots$ \\
\hline 9 & UGC 4261 & 10.65 & $<0.35$ & 1.22 & 1.56 & $\ldots$ & $\ldots$ & $\ldots$ \\
\hline 10 & NGC 2535 & 10.62 & 0.28 & 2.13 & 5.97 & $\cdots$ & $\cdots$ & $\cdots$ \\
\hline 11 & NGC 2543 & 9.90 & $0.33^{\mathrm{b}}$ & 2.78 & 6.14 & $3.04 \pm 0.31$ & $\ldots$ & $\cdots$ \\
\hline 12 & NGC 2537 & 9.36 & 0.27 & 3.12 & 5.78 & $\ldots$ & $\ldots$ & $\cdots$ \\
\hline 13 & IC 2233 & 8.62 & 0.037 & 0.93 & 1.47 & $\cdots$ & $\ldots$ & $\ldots$ \\
\hline 14 & IC 2239 & 10.98 & $<0.57$ & 2.94 & 5.30 & $\ldots$ & $\cdots$ & $\ldots$ \\
\hline 15 & UGC 4286 & 10.63 & $<0.59$ & 0.87 & 1.81 & $\ldots$ & $\ldots$ & $\ldots$ \\
\hline 16 & UGC 4306 & 10.20 & $<0.36$ & 3.59 & 6.77 & $\ldots$ & $\ldots$ & $\cdots$ \\
\hline 17 & NGC 2552 & 8.53 & 0.056 & 0.61 & 1.50 & $\cdots$ & $\ldots$ & $\cdots$ \\
\hline 18 & IC 2339 & 10.51 & 0.15 & 1.65 & 2.88 & $\ldots$ & $0.85 \pm 0.13$ & $\ldots$ \\
\hline 19 & IRAS $08234+1054 B$ & 11.73 & $<0.32$ & 1.82 & 3.19 & $\ldots$ & $\ldots$ & $\cdots$ \\
\hline 20 & IRAS $08269+1514$ & 11.05 & $<0.32$ & 0.80 & 2.30 & $\cdots$ & $\cdots$ & $\cdots$ \\
\hline 21 & NGC 2604 & 10.00 & $<0.40$ & 1.31 & 2.85 & $\cdots$ & $\cdots$ & $\cdots$ \\
\hline 22 & NGC 2608 & 10.15 & $0.25^{\mathrm{b}}$ & 2.30 & 5.97 & $2.19 \pm 0.14$ & $\cdots$ & $\cdots$ \\
\hline 23 & MK 92 & 10.59 & $0.35^{\mathrm{b}}$ & 2.53 & 3.88 & $\ldots$ & $\ldots$ & $\ldots$ \\
\hline 24 & NGC 2623 & 11.49 & 1.35 & 24.33 & 28.24 & $\cdots$ & $\cdots$ & $\cdots$ \\
\hline 25 & CGCG 120-018 & 10.99 & $0.28^{\mathrm{b}}$ & 3.18 & 4.51 & $\ldots$ & $\ldots$ & $\cdots$ \\
\hline 26 & NGC 2644 & 9.69 & $<0.31$ & 1.53 & 3.62 & $\cdots$ & $\cdots$ & $\cdots$ \\
\hline 27 & UGC 4572 & 10.29 & $<0.27$ & 1.01 & 2.09 & $\cdots$ & $\cdots$ & $\cdots$ \\
\hline 28 & UGC 4653 & 11.69 & $<0.30$ & 2.14 & 4.55 & $1.58 \pm 0.20$ & $\cdots$ & $\cdots$ \\
\hline 29 & IRAS $08512+2727$ & 11.59 & $<0.32$ & 0.67 & 1.81 & $\ldots$ & $\cdots$ & $\cdots$ \\
\hline 30 & OJ $287^{\mathrm{c}}$ & 13.19 & $<0.71$ & 0.94 & 1.31 & $2.62 \pm 0.15$ & $3.62 \pm 0.10$ & $3.92 \pm 0.11$ \\
\hline 31 & IRAS $08538+4256$ & 11.24 & $0.39^{\mathrm{b}}$ & 4.65 & 6.44 & $\ldots$ & $\ldots$ & $\ldots$ \\
\hline 32 & IRAS $08550+3908$ & 11.80 & $<0.30$ & 1.23 & 1.54 & $\cdots$ & $\cdots$ & $\cdots$ \\
\hline 33 & NGC 2718 & 10.68 & $0.58^{\mathrm{b}}$ & 4.03 & 7.01 & $\cdots$ & $\cdots$ & $\cdots$ \\
\hline 34 & NGC 2712 & 9.98 & $0.30^{\mathrm{b}}$ & 2.19 & 5.29 & $2.26 \pm 0.11$ & $0.69 \pm 0.09$ & $\ldots$ \\
\hline 35 & NGC 2719 & 10.02 & 0.08 & 1.85 & 2.61 & $\ldots$ & $\ldots$ & $\ldots$ \\
\hline 36 & IRAS $08572+3915$ & 12.02 & 1.44 & 7.53 & 4.59 & $\cdots$ & $\cdots$ & $\cdots$ \\
\hline 37 & IRAS $08579+3447$ & 11.82 & $<0.27$ & 2.78 & 4.82 & $\cdots$ & $\cdots$ & $\cdots$ \\
\hline 38 & NGC 2731 & 10.16 & $<0.55$ & 3.11 & 5.20 & $\cdots$ & $\cdots$ & $\ldots$ \\
\hline 39 & NGC 2730 & 10.34 & $<0.34$ & 0.74 & 2.20 & $\ldots$ & $\cdots$ & $\cdots$ \\
\hline 40 & IC 2431 & 11.82 & $0.54^{\mathrm{b}}$ & 4.43 & 7.63 & $\ldots$ & $\ldots$ & $\cdots$ \\
\hline 41 & NGC 2750 & 10.33 & $0.59^{\mathrm{b}}$ & 3.93 & 7.68 & $2.66 \pm 0.15$ & $0.86 \pm 0.08$ & $\ldots$ \\
\hline 42 & IC 2434 & 10.88 & $<0.25$ & 1.70 & 3.44 & $\ldots$ & $\ldots$ & $\cdots$ \\
\hline 43 & NGC 2761 & 11.36 & $0.46^{\mathrm{b}}$ & 3.97 & 7.70 & $1.61 \pm 0.11$ & $\ldots$ & $\ldots$ \\
\hline 44 & NGC 2773 & 10.84 & $0.39^{\mathrm{b}}$ & 2.84 & 5.26 & $\cdots$ & $\cdots$ & $\cdots$ \\
\hline 45 & NGC 2776 & 10.31 & $0.32^{\mathrm{b}}$ & 3.42 & 9.17 & $3.72 \pm 0.13$ & $1.20 \pm 0.11$ & $\ldots$ \\
\hline 46 & NGC 2789 & 10.95 & $<0.33$ & 2.23 & 5.16 & $1.57 \pm 0.12$ & $\ldots$ & $\ldots$ \\
\hline 47 & IRAS $09121+3908$ & 10.03 & $<0.25$ & 1.18 & 2.64 & $\ldots$ & $\cdots$ & $\ldots$ \\
\hline 48 & NGC 2824 & 9.87 & $<0.33$ & 1.12 & 1.83 & $\ldots$ & $\cdots$ & $\cdots$ \\
\hline 49 & IRAS $09184+4356$ & 11.25 & $<0.25$ & 1.32 & 2.83 & $\ldots$ & $\ldots$ & $\ldots$ \\
\hline 50 & CGCG 238-041 & 10.99 & $<0.31$ & 0.60 & 1.97 & $\cdots$ & $\cdots$ & $\cdots$ \\
\hline 51 & UGC 4985 & 11.12 & $<0.33$ & 0.84 & 2.34 & $\ldots$ & $\cdots$ & $\cdots$ \\
\hline 52 & NGC 2854 & 9.54 & 0.20 & 2.00 & 2.93 & $2.57 \pm 0.24$ & $\cdots$ & $\cdots$ \\
\hline 53 & UGC 5046 & 10.67 & $<0.49$ & 3.01 & 5.09 & $\ldots$ & $\cdots$ & $\cdots$ \\
\hline 54 & UGC 5055 & 10.95 & $<0.33$ & 1.53 & 3.14 & $\cdots$ & $\cdots$ & $\cdots$ \\
\hline 55 & NGC 2893 & 9.78 & $0.59^{\mathrm{b}}$ & 2.54 & 3.83 & $\cdots$ & $\cdots$ & $\cdots$ \\
\hline 56 & MCG 3-24-062 & 10.52 & $<0.29$ & 1.24 & 3.49 & $\cdots$ & $\cdots$ & $\cdots$ \\
\hline 57 & CGCG 238-066 & 11.20 & $0.46^{\mathrm{b}}$ & 1.75 & 2.36 & $\ldots$ & $\cdots$ & $\cdots$ \\
\hline 58 & UGC 5097 & 10.68 & $0.31^{\mathrm{b}}$ & 2.83 & 4.81 & $\ldots$ & $\ldots$ & $\cdots$ \\
\hline 59 & CGCG 289-012 & 11.31 & $<0.26$ & 1.49 & 3.24 & $\ldots$ & $\ldots$ & $\cdots$ \\
\hline 60 & MCG 8-18-013 & 11.29 & 0.56 & 5.87 & 8.40 & $\cdots$ & $\cdots$ & $\cdots$ \\
\hline 61 & CGCG 181-068 & 10.86 & $<0.28$ & 1.19 & 3.26 & $\ldots$ & $\ldots$ & $\ldots$ \\
\hline 62 & NGC 2936 & 10.95 & 0.20 & 2.15 & 5.03 & $\ldots$ & $\ldots$ & $\ldots$ \\
\hline 63 & NGC 2955 & 10.99 & $<0.31$ & 1.61 & 4.51 & $1.10 \pm 0.10$ & $\cdots$ & $\cdots$ \\
\hline 64 & CGCG 182-010 & 11.40 & $0.40^{\mathrm{b}}$ & 1.94 & 3.41 & $\cdots$ & $\ldots$ & $\ldots$ \\
\hline 65 & UGC 5228 & 9.78 & $<0.27$ & 1.63 & 3.69 & $\ldots$ & $\ldots$ & $\ldots$ \\
\hline 66 & IRAS $09438+1141$ & 11.46 & $<0.32$ & 1.16 & 2.89 & $\ldots$ & $\cdots$ & $\cdots$ \\
\hline 67 & NGC 3015 & 11.02 & $<0.33$ & 2.16 & 4.28 & $\cdots$ & $\cdots$ & $\cdots$ \\
\hline 68 & MCG 2-25-039 & 10.64 & $<0.30$ & 1.45 & 3.23 & $\ldots$ & $\ldots$ & $\cdots$ \\
\hline 69 & NGC 3020 & 9.37 & $0.28^{\mathrm{b}}$ & 1.98 & 3.38 & $\ldots$ & $\ldots$ & $\ldots$ \\
\hline 70 & NGC 3049 & 9.50 & 0.42 & 2.71 & 4.34 & $1.91 \pm 0.17$ & $1.06 \pm 0.11$ & $\cdots$ \\
\hline 71 & NGC 3055 & 10.04 & $0.64^{\mathrm{b}}$ & 4.05 & 8.48 & $\ldots$ & $\ldots$ & $\ldots$ \\
\hline
\end{tabular}


TABLE 7 - Continued

\begin{tabular}{|c|c|c|c|c|c|c|c|c|}
\hline SFRS & Name & $\begin{array}{c}\mathrm{L}(\mathrm{TIR}) \\
\left(\mathrm{L}_{\odot}\right)\end{array}$ & $\underset{(\mathrm{Jy})}{\operatorname{MIPS~F}(24)^{\mathrm{a}}}$ & $\begin{array}{c}\text { IRAS F(60) } \\
(\mathrm{Jy})\end{array}$ & $\begin{array}{c}\text { IRAS F(100) } \\
(\mathrm{Jy})\end{array}$ & $\underset{(\mathrm{Jy})}{\text { Planck F(350) }}$ & $\underset{(\mathrm{Jy})}{\text { Planck F(550) }}$ & $\begin{array}{c}\text { Planck (F850) } \\
(\mathrm{Jy})\end{array}$ \\
\hline 72 & IC 2520 & 9.97 & $0.48^{\mathrm{b}}$ & 3.42 & 6.77 & $\ldots$ & $\ldots$ & $\ldots$ \\
\hline 73 & UGC 5403 & 10.00 & $0.39^{\mathrm{b}}$ & 2.71 & 4.28 & $\ldots$ & $\ldots$ & $\ldots$ \\
\hline 74 & UGC 5459 & 9.79 & $0.27^{\mathrm{b}}$ & 2.17 & 5.00 & $2.95 \pm 0.16$ & $1.02 \pm 0.10$ & $\ldots$ \\
\hline 75 & MCG 5-24-022 & 10.71 & $<0.26$ & 0.97 & 2.60 & $\begin{array}{c}2.00+0 \\
\ldots\end{array}$ & $\begin{array}{c}1.02 \pm 0 \\
\ldots\end{array}$ & $\ldots$ \\
\hline 76 & IC 2551 & 11.06 & $0.82^{\mathrm{b}}$ & 3.84 & 4.24 & $\ldots$ & $\ldots$ & $\ldots$ \\
\hline 77 & IRAS $10106+2745$ & 11.41 & $<0.26$ & 0.81 & 2.21 & $2.18 \pm 0.28$ & $\ldots$ & $\ldots$ \\
\hline 78 & NGC 3162 & 9.98 & $0.52^{\mathrm{b}}$ & 2.93 & 6.48 & $2.69 \pm 0.13$ & $1.08 \pm 0.08$ & $\ldots$ \\
\hline 79 & IRAS $10120+1653$ & 12.21 & $<0.37$ & 0.86 & 1.88 & $\ldots$ & $\ldots$ & $\ldots$ \\
\hline 80 & NGC 3190 & 10.05 & 0.25 & 3.33 & 9.84 & $4.45 \pm 0.28$ & $1.52 \pm 0.12$ & $\ldots$ \\
\hline 81 & IC 602 & 10.55 & $<0.33$ & 2.84 & 5.71 & $1.29 \pm 0.12$ & $\ldots$ & $\ldots$ \\
\hline 82 & NGC 3191 & 11.15 & $<0.29$ & 1.70 & 3.81 & $1.00 \pm 0.14$ & $\ldots$ & $\ldots$ \\
\hline 83 & NGC 3206 & 9.40 & $<0.10$ & 1.07 & 2.14 & $\ldots$ & $\ldots$ & $\ldots$ \\
\hline 84 & UGC 5613 & 11.44 & 0.32 & 4.20 & 8.27 & $\ldots$ & $\ldots$ & $\ldots$ \\
\hline 85 & UGC 5644 & 10.67 & 0.07 & 0.66 & 1.34 & $\ldots$ & $\ldots$ & $\ldots$ \\
\hline 86 & NGC 3245 & 9.40 & $0.18^{\mathrm{b}}$ & 1.98 & 3.17 & $\ldots$ & $\ldots$ & $\ldots$ \\
\hline 87 & IRAS $10246+2042$ & 10.78 & $<0.39$ & 2.58 & 3.86 & $\ldots$ & $\ldots$ & $\ldots$ \\
\hline 88 & MCG 7-22-012 & 10.41 & 0.07 & 1.43 & 3.76 & $0.86 \pm 0.11$ & $\ldots$ & $\ldots$ \\
\hline 89 & IRAS $10276+1119$ & 11.83 & $<0.51$ & 0.79 & $<3.02$ & $\begin{array}{c}.00 \\
\ldots\end{array}$ & $\ldots$ & $\ldots$ \\
\hline 90 & NGC 3265 & 9.52 & 0.28 & 2.21 & 3.40 & $\ldots$ & $\ldots$ & $\ldots$ \\
\hline 91 & UGC 5713 & 10.66 & $<0.39$ & 0.91 & 1.13 & $\ldots$ & $\ldots$ & $\ldots$ \\
\hline 92 & NGC 3274 & 8.48 & 0.06 & 0.99 & 1.86 & $\ldots$ & $\ldots$ & $\ldots$ \\
\hline 93 & UGC 5720 & 9.76 & 0.84 & 4.79 & 5.49 & $\ldots$ & $\ldots$ & $\ldots$ \\
\hline 94 & KUG $1031+351$ & 11.93 & $0.31^{\mathrm{b}}$ & 2.68 & 5.02 & $1.06 \pm 0.10$ & $\ldots$ & $\ldots$ \\
\hline 95 & NGC 3306 & 10.37 & $0.41^{\mathrm{b}}$ & 2.89 & 5.26 & $\ldots$ & $\ldots$ & $\ldots$ \\
\hline 96 & NGC 3323 & 10.65 & $<0.27$ & 1.50 & 3.30 & $\ldots$ & $\ldots$ & $\ldots$ \\
\hline 97 & IC 2598 & 10.92 & $0.35^{\mathrm{b}}$ & 3.24 & 5.49 & $\ldots$ & $\ldots$ & $\ldots$ \\
\hline 98 & NGC 3338 & 9.91 & 0.37 & $\begin{array}{l}3.44 \\
3.43\end{array}$ & $\begin{array}{r}0.49 \\
10.32\end{array}$ & $6.21 \pm 0.23$ & $2.55 \pm 0.14$ & $0.55 \pm 0.06$ \\
\hline 99 & NGC 3353 & 9.63 & 0.91 & 5.18 & 6.65 & $1.20 \pm 0.11$ & $\ldots$ & $\ldots$ \\
\hline 100 & UGC 5881 & 10.71 & $<0.30$ & 1.76 & 2.45 & $\ldots$ & $\ldots$ & $\ldots$ \\
\hline 101 & NGC 3370 & 9.85 & 0.38 & 3.43 & 9.13 & $3.73 \pm 0.16$ & $1.41 \pm 0.09$ & $\ldots$ \\
\hline 102 & NGC 3381 & 9.71 & $<0.25$ & 1.40 & 3.95 & $1.48 \pm 0.11$ & $\ldots$ & $\ldots$ \\
\hline 103 & UGC 5941 & 11.02 & $<0.31$ & 2.52 & 4.76 & $\ldots$ & $\ldots$ & $\ldots$ \\
\hline 104 & NGC 3413 & 8.92 & 0.06 & 1.03 & 1.96 & $\ldots$ & $\ldots$ & $\ldots$ \\
\hline 105 & NGC 3408 & 9.41 & 0.12 & 0.91 & 1.55 & $0.81 \pm 0.10$ & $\ldots$ & $\ldots$ \\
\hline 106 & NGC 3430 & 11.40 & 0.17 & 2.87 & 8.32 & $5.99 \pm 0.55$ & $1.60 \pm 0.20$ & $\ldots$ \\
\hline 107 & CGCG 95-055 & 9.42 & 0.22 & 1.88 & 1.72 & $\ldots$ & $\ldots$ & $\ldots$ \\
\hline 108 & IRAS $10565+2448$ & 11.95 & 0.95 & 12.17 & 14.34 & $1.84 \pm 0.10$ & $\ldots$ & $\ldots$ \\
\hline 109 & UGC 6074 & 10.28 & $0.78^{\mathrm{b}}$ & 3.48 & 4.68 & $\ldots$ & $\ldots$ & $\ldots$ \\
\hline 110 & NGC 3495 & 9.74 & $0.60^{\mathrm{b}}$ & 3.36 & 8.80 & $4.99 \pm 0.26$ & $1.80 \pm 0.12$ & $\ldots$ \\
\hline 111 & UGC 6103 & 10.88 & $0.38^{\mathrm{b}}$ & 2.47 & 4.12 & $\ldots$ & $\ldots$ & $\ldots$ \\
\hline 112 & MCG 7-23-019 & 11.57 & 0.25 & 6.06 & 10.66 & $0.91 \pm 0.14$ & $\ldots$ & $\ldots$ \\
\hline 113 & UGC 6135 & 10.89 & $<0.25$ & 1.73 & 5.04 & $1.01 \pm 0.09$ & $\ldots$ & $\ldots$ \\
\hline 114 & CGCG 241-078 & 10.93 & $<0.28$ & 2.42 & 3.38 & $\ldots$ & $\ldots$ & $\ldots$ \\
\hline 115 & IRAS $11069+2711$ & 11.76 & 0.11 & 2.14 & 4.18 & $\ldots$ & $\ldots$ & $\ldots$ \\
\hline 116 & IC 676 & 9.90 & 0.52 & 3.05 & 4.76 & $\ldots$ & $\ldots$ & $\ldots$ \\
\hline 117 & IRAS $11102+3026$ & 11.02 & 0.10 & 2.27 & 4.04 & $\ldots$ & $\ldots$ & $\ldots$ \\
\hline 118 & IC 2637 & 11.00 & 0.17 & 1.95 & 3.42 & $\ldots$ & $\ldots$ & $\ldots$ \\
\hline 119 & MCG 9-19-013 & 11.37 & $<0.25$ & 0.90 & 2.46 & $\ldots$ & $\ldots$ & $\ldots$ \\
\hline 120 & 7ZW 384 & 11.66 & 0.09 & 0.94 & 2.28 & $\ldots$ & $\ldots$ & $\ldots$ \\
\hline 121 & IRAS $11167+5351$ & 11.95 & 0.13 & 1.39 & 2.44 & $\ldots$ & $\ldots$ & $\ldots$ \\
\hline 122 & NGC 3633 & 10.09 & $0.70^{\mathrm{b}}$ & 3.04 & 5.36 & $\ldots$ & $\ldots$ & $\ldots$ \\
\hline 123 & NGC 3652 & 9.39 & 0.19 & 2.38 & 6.03 & $1.60 \pm 0.15$ & $\ldots$ & $\ldots$ \\
\hline 124 & NGC 3656 & 10.08 & 0.15 & 2.39 & 5.45 & $1.50 \pm 0.12$ & $\ldots$ & $\ldots$ \\
\hline 125 & NGC 3659 & 9.47 & 0.13 & 1.65 & 3.95 & $1.55 \pm 0.12$ & $\ldots$ & $\ldots$ \\
\hline 126 & NGC 3664 & 9.37 & 0.08 & 0.90 & 1.85 & $2.83 \pm 0.54$ & $1.66 \pm 0.25$ & $\ldots$ \\
\hline 127 & NGC 3666 & 9.57 & 0.22 & 2.92 & 8.59 & $2.84 \pm 0.12$ & $0.99 \pm 0.07$ & $\ldots$ \\
\hline 128 & IC 691 & 9.42 & 0.46 & 3.61 & 4.79 & $\begin{array}{c}2.0+1 . \\
\ldots\end{array}$ & $\begin{array}{c}0.00+0 \\
\ldots\end{array}$ & $\ldots$ \\
\hline 129 & NGC 3686 & 9.92 & 0.48 & 3.82 & 10.31 & $4.09 \pm 0.12$ & $1.06 \pm 0.10$ & $\ldots$ \\
\hline 130 & UGC 6469 & 10.71 & 0.12 & $\begin{array}{l}.02 \\
1.66\end{array}$ & $\begin{array}{r}10.01 \\
2.88\end{array}$ & & & $\ldots$ \\
\hline 131 & IC 694 & 11.91 & 17.32 & 105.80 & 111.20 & $9.62 \pm 0.17$ & $2.56 \pm 0.09$ & $0.64 \pm 0.06$ \\
\hline 132 & IC 698 & 11.07 & 0.32 & 3.74 & 7.11 & $\ldots$ & $\ldots$ & $\ldots$ \\
\hline 133 & IRAS $11267+1558$ & 12.25 & 0.039 & 0.76 & $<2.07$ & $\ldots$ & $\ldots$ & $\ldots$ \\
\hline 134 & NGC 3705 & 9.68 & 0.38 & 3.69 & 10.42 & $5.83 \pm 0.31$ & $2.08 \pm 0.13$ & $\ldots$ \\
\hline 135 & MCG 3-29-061 & 10.54 & 0.26 & 2.64 & 4.05 & & $\begin{array}{c}2.00+0.00 \\
\ldots\end{array}$ & $\ldots$ \\
\hline 136 & NGC 3720 & 10.91 & 0.18 & 2.55 & 6.11 & $1.67 \pm 0.17$ & $\ldots$ & $\ldots$ \\
\hline 137 & NGC 3729 & 9.62 & 0.40 & 2.85 & 7.44 & $2.32 \pm 0.13$ & $0.60 \pm 0.07$ & $\ldots$ \\
\hline 138 & MCG 10-17-019 & 11.05 & 0.12 & 1.39 & 4.08 & $1.17 \pm 0.11$ & $\ldots$ & $\ldots$ \\
\hline 139 & NGC 3758 & 10.97 & 0.24 & 1.43 & 2.43 & $\ldots$ & $\ldots$ & $\ldots$ \\
\hline 140 & UGC 6583 & 10.97 & $<0.51$ & 1.98 & 4.39 & $1.14 \pm 0.10$ & $\ldots$ & $\ldots$ \\
\hline 141 & MCG 1-30-003 & 11.05 & 0.24 & 1.97 & 3.45 & $\ldots$ & $\ldots$ & $\ldots$ \\
\hline 142 & NGC 3769 & 9.52 & 0.22 & 2.53 & 6.76 & $3.03 \pm 0.11$ & $1.05 \pm 0.07$ & $\ldots$ \\
\hline 143 & NGC 3773 & 8.97 & 0.14 & 1.51 & 1.84 & $\ldots$ & $\ldots$ & $\ldots$ \\
\hline 144 & NGC 3781 & 11.31 & 0.71 & 7.07 & 9.99 & $1.85 \pm 0.19$ & $0.55 \pm 0.08$ & $\ldots$ \\
\hline
\end{tabular}


Ashby et al.

TABLE 7 Continued

\begin{tabular}{|c|c|c|c|c|c|c|c|c|}
\hline SFRS & Name & $\begin{array}{c}\mathrm{L}(\mathrm{TIR}) \\
\left(\mathrm{L}_{\odot}\right)\end{array}$ & $\begin{array}{c}\operatorname{MIPS~F}(24)^{\mathrm{a}} \\
(\mathrm{Jy})\end{array}$ & $\begin{array}{c}\text { IRAS F(60) } \\
(\mathrm{Jy})\end{array}$ & $\begin{array}{c}\text { IRAS F(100) } \\
\text { (Jy) }\end{array}$ & $\begin{array}{c}\text { Planck F(350) } \\
\text { (Jy) }\end{array}$ & $\underset{(J y)}{\text { Planck F }}(550)$ & $\underset{(\mathrm{Jy})}{\text { Planck (F850) }}$ \\
\hline 145 & UGC 6625 & 11.16 & 0.12 & 1.23 & 3.36 & & $\cdots$ & $\cdots$ \\
\hline 146 & NGC 3808A & 11.06 & 0.20 & 2.78 & 6.02 & $1.15 \pm 0.17$ & $\cdots$ & $\ldots$ \\
\hline 147 & NGC 3811 & 10.45 & 0.20 & 2.49 & 5.73 & $1.92 \pm 0.11$ & $0.71 \pm 0.09$ & $\ldots$ \\
\hline 148 & NGC 3822 & 11.03 & 0.28 & 3.19 & 6.97 & $3.39 \pm 0.39$ & $\ldots$ & $\ldots$ \\
\hline 149 & UGC 6665 & 10.91 & 0.62 & 3.89 & 4.51 & $\ldots$ & $\ldots$ & $\ldots$ \\
\hline 150 & MCG 3-30-051 & 10.77 & 0.18 & 1.82 & 4.02 & $\ldots$ & $\ldots$ & $\ldots$ \\
\hline 151 & NGC 3839 & 11.04 & 0.31 & 4.18 & 7.68 & $\ldots$ & $\ldots$ & $\ldots$ \\
\hline 152 & UGC 6732 & 10.16 & 0.27 & 1.41 & 2.00 & $\ldots$ & $\ldots$ & $\ldots$ \\
\hline 153 & IC 730 & 10.93 & 0.23 & 3.24 & 5.86 & $\cdots$ & $\cdots$ & $\ldots$ \\
\hline 154 & IC 732 & 11.13 & $0.33^{\mathrm{b}}$ & 3.43 & 6.08 & $1.63 \pm 0.12$ & $0.78 \pm 0.08$ & $\ldots$ \\
\hline 155 & NGC 3912 & 9.75 & 0.26 & 3.40 & 6.56 & $1.49 \pm 0.08$ & $\ldots$ & $\ldots$ \\
\hline 156 & NGC 3928 & 9.47 & 0.31 & 2.67 & 5.46 & $\ldots$ & $\ldots$ & $\ldots$ \\
\hline 157 & NGC 3934 & 10.33 & 0.11 & 2.25 & 3.60 & $\ldots$ & $\ldots$ & $\ldots$ \\
\hline 158 & UGC 6865 & 10.86 & 0.21 & 2.31 & 4.94 & $1.52 \pm 0.12$ & $\ldots$ & $\ldots$ \\
\hline 159 & UGC 6901 & 11.00 & 0.17 & 2.01 & 5.13 & $1.15 \pm 0.11$ & $\ldots$ & $\ldots$ \\
\hline 160 & CGCG 013-010 & 11.57 & $<0.25$ & 3.29 & 7.53 & $1.45 \pm 0.11$ & $\ldots$ & $\ldots$ \\
\hline 161 & NGC 3991 & 10.43 & 0.24 & 2.56 & 4.84 & $3.56 \pm 0.17$ & $1.10 \pm 0.07$ & $\ldots$ \\
\hline 162 & NGC 4004 & 10.61 & 0.36 & 3.63 & 6.71 & $1.84 \pm 0.11$ & $0.62 \pm 0.08$ & $\ldots$ \\
\hline 163 & NGC 4014 & 10.62 & 0.23 & 2.46 & 6.22 & $\ldots$ & $\ldots$ & $\ldots$ \\
\hline 164 & NGC 4010 & 9.50 & 0.21 & 2.40 & 6.42 & $4.08 \pm 0.24$ & $1.53 \pm 0.11$ & $0.38 \pm 0.05$ \\
\hline 165 & NGC 4018 & 10.77 & 0.17 & 2.35 & 6.87 & $1.16 \pm 0.09$ & $\ldots$ & $\ldots$ \\
\hline 166 & NGC 4020 & 8.94 & 0.09 & 0.96 & 2.47 & $1.10 \pm 0.09$ & $\ldots$ & $\ldots$ \\
\hline 167 & IRAS $11571+3003$ & 11.16 & 0.050 & 0.86 & 1.88 & $\ldots$ & $\ldots$ & $\ldots$ \\
\hline 168 & UGC 7017 & 10.59 & 0.29 & 3.78 & 7.46 & $1.59 \pm 0.11$ & $\cdots$ & $\ldots$ \\
\hline 169 & UGC 7016 & 10.85 & 0.12 & 1.46 & 3.46 & $\ldots$ & $\ldots$ & $\ldots$ \\
\hline 170 & MCG 3-31-030 & 8.72 & 0.054 & 0.67 & 1.84 & $\ldots$ & $\ldots$ & $\ldots$ \\
\hline 171 & NGC 4062 & 9.67 & 0.35 & 2.68 & 10.16 & $5.91 \pm 0.14$ & $1.76 \pm 0.09$ & $0.39 \pm 0.06$ \\
\hline 172 & NGC 4064 & 8.95 & 0.34 & 3.29 & 6.81 & $\ldots$ & $\ldots$ & $\ldots$ \\
\hline 173 & CGCG 098-059 & 11.14 & 0.34 & 3.41 & 7.31 & $\ldots$ & $\ldots$ & $\ldots$ \\
\hline 174 & NGC 4116 & 9.38 & 0.18 & 1.89 & 5.56 & $3.33 \pm 0.14$ & $1.28 \pm 0.10$ & $\ldots$ \\
\hline 175 & NGC 4136 & 9.36 & 0.12 & 2.12 & 5.42 & $3.13 \pm 0.10$ & $1.12 \pm 0.08$ & $0.59 \pm 0.07$ \\
\hline 176 & NGC 4150 & 8.87 & 0.07 & 1.24 & 2.46 & $\ldots$ & $\ldots$ & $\ldots$ \\
\hline 177 & IRAS $12086+1441$ & 9.06 & $<0.47$ & 1.26 & 1.78 & $\ldots$ & $\ldots$ & $\ldots$ \\
\hline 178 & NGC 4162 & 10.31 & 0.23 & 2.45 & 6.61 & $3.61 \pm 0.35$ & $1.05 \pm 0.18$ & $\ldots$ \\
\hline 179 & NGC 4178 & 9.67 & 0.39 & 4.02 & 9.47 & $6.19 \pm 0.15$ & $1.75 \pm 0.11$ & $\ldots$ \\
\hline 180 & IRAS $12112+0305$ & 12.15 & 0.34 & 8.36 & 9.91 & $\cdots$ & $\cdots$ & $\ldots$ \\
\hline 181 & NGC 4189 & 9.65 & 0.39 & 3.29 & 8.70 & $3.05 \pm 0.16$ & $\ldots$ & $\ldots$ \\
\hline 182 & NGC 4194 & 10.94 & 3.83 & 22.79 & 25.94 & $2.02 \pm 0.11$ & $\ldots$ & $\ldots$ \\
\hline 183 & NGC 4204 & 8.54 & 0.08 & 1.01 & 2.04 & $2.01 \pm 0.21$ & $\ldots$ & $\ldots$ \\
\hline 184 & NGC 4207 & 9.54 & 0.21 & 3.03 & 7.46 & $1.41 \pm 0.11$ & $\ldots$ & $\ldots$ \\
\hline 185 & UGC 7286 & 10.55 & 0.06 & 0.90 & 1.61 & $\ldots$ & $\ldots$ & $\ldots$ \\
\hline 186 & NGC 4234 & 9.77 & 0.14 & 1.66 & 3.88 & $0.96 \pm 0.13$ & $\ldots$ & $\ldots$ \\
\hline 187 & NGC 4237 & 9.64 & 0.28 & 2.81 & 9.19 & $4.08 \pm 0.15$ & $0.81 \pm 0.08$ & $\ldots$ \\
\hline 188 & NGC $4244^{\mathrm{d}}$ & 8.69 & 0.40 & 4.20 & 16.06 & $7.92 \pm 0.53$ & $3.54 \pm 0.19$ & $0.90 \pm 0.09$ \\
\hline 189 & NGC 4253 & 10.83 & 1.19 & 4.06 & 4.25 & $\ldots$ & $\ldots$ & $\ldots$ \\
\hline 190 & MCG 3-32-005 & 9.21 & 0.24 & 2.78 & 5.35 & $\ldots$ & $\cdots$ & $\ldots$ \\
\hline 191 & NGC 4290 & 10.33 & 0.45 & 3.90 & 8.46 & $2.62 \pm 0.17$ & $0.80 \pm 0.08$ & $\ldots$ \\
\hline 192 & NGC 4294 & 9.43 & 0.21 & 2.75 & 5.56 & $2.22 \pm 0.21$ & $0.96 \pm 0.10$ & $\ldots$ \\
\hline 193 & NGC 4314 & 9.51 & 0.29 & 3.44 & 7.02 & $1.93 \pm 0.23$ & $\ldots$ & $\ldots$ \\
\hline 194 & NGC 4385 & 10.14 & 0.96 & 4.66 & 6.13 & $1.91 \pm 0.13$ & $\ldots$ & $\ldots$ \\
\hline 195 & NGC 4395 & 8.71 & $0.51^{\mathrm{e}}$ & 4.21 & 12.90 & $6.20 \pm 0.34$ & $2.87 \pm 0.19$ & $0.81 \pm 0.10$ \\
\hline 196 & NGC 4396 & 9.27 & 0.13 & 1.27 & 3.84 & $2.58 \pm 0.13$ & $\ldots$ & $\ldots$ \\
\hline 197 & NGC 4412 & 10.03 & 0.37 & 3.00 & 5.90 & $\ldots$ & $\ldots$ & $\ldots$ \\
\hline 198 & NGC 4418 & 11.02 & $9.57^{\mathrm{b}}$ & 43.86 & 33.78 & $3.33 \pm 0.16$ & $1.24 \pm 0.11$ & $\ldots$ \\
\hline 199 & NGC 4420 & 9.57 & 0.23 & 2.70 & 7.08 & $2.12 \pm 0.18$ & $\ldots$ & $\ldots$ \\
\hline 200 & NGC 4424 & 8.56 & 0.30 & 2.89 & 5.48 & $\ldots$ & $\ldots$ & $\ldots$ \\
\hline 201 & NGC 4435 & 9.03 & 0.11 & 1.88 & 2.29 & $4.56 \pm 0.26$ & $1.07 \pm 0.13$ & $\ldots$ \\
\hline 202 & NGC $4438^{\mathrm{d}}$ & 9.73 & 0.23 & 4.28 & 12.05 & $4.56 \pm 0.26$ & $1.07 \pm 0.13$ & $\ldots$ \\
\hline 203 & NGC 4448 & 9.36 & 0.18 & 1.60 & 4.97 & $3.20 \pm 0.17$ & $0.82 \pm 0.11$ & $\ldots$ \\
\hline 204 & $3 \mathrm{C} 273$ & 12.56 & 0.52 & 2.22 & 2.91 & $4.07 \pm 0.13$ & $6.94 \pm 0.17$ & $8.60 \pm 0.16$ \\
\hline 205 & NGC 4470 & 9.32 & 0.14 & 1.85 & 4.49 & $1.18 \pm 0.17$ & $\cdots$ & $\cdots$ \\
\hline 206 & IRAS $12274+0018$ & 9.44 & 0.06 & 0.81 & 1.08 & $\cdots$ & $\cdots$ & $\cdots$ \\
\hline 207 & NGC 4491 & 9.29 & 0.30 & 2.64 & 3.31 & & $\ldots$ & $\ldots$ \\
\hline 208 & NGC 4500 & 10.55 & 0.45 & 3.92 & 6.84 & $0.97 \pm 0.13$ & $\ldots$ & $\ldots$ \\
\hline 209 & NGC 4495 & 10.82 & 0.26 & 3.16 & 7.00 & $1.84 \pm 0.13$ & $\ldots$ & $\ldots$ \\
\hline 210 & IC 3476 & 9.20 & 0.14 & 1.30 & 3.15 & $\ldots$ & $\ldots$ & $\ldots$ \\
\hline 211 & NGC 4509 & 8.65 & 0.06 & 0.60 & $<2.16$ & $\ldots$ & $\ldots$ & $\ldots$ \\
\hline 212 & NGC 4519 & 9.58 & 0.51 & 3.76 & 6.56 & $2.85 \pm 0.14$ & $1.10 \pm 0.10$ & $\ldots$ \\
\hline 213 & NGC 4548 & 9.73 & 0.48 & 2.61 & 11.10 & $6.46 \pm 0.25$ & $2.01 \pm 0.11$ & $\ldots$ \\
\hline 214 & IRAS $12337+5044$ & 11.19 & 0.11 & 1.41 & 3.06 & & $\ldots$ & $\ldots$ \\
\hline 215 & IC 3581 & 10.99 & 0.29 & 3.80 & 4.82 & $1.33 \pm 0.17$ & $\ldots$ & $\ldots$ \\
\hline 216 & NGC 4592 & 8.97 & 0.12 & 2.18 & 4.76 & $2.32 \pm 0.13$ & $1.08 \pm 0.12$ & $\ldots$ \\
\hline 217 & NGC 4607 & 9.57 & 0.25 & 2.75 & 7.84 & $2.79 \pm 0.31$ & $\ldots$ & $\ldots$ \\
\hline 218 & NGC 4625 & 8.75 & 0.12 & 1.22 & 3.81 & $\ldots$ & $\ldots$ & $\ldots$ \\
\hline
\end{tabular}


TABLE 7 - Continued

\begin{tabular}{|c|c|c|c|c|c|c|c|c|}
\hline SFRS & Name & $\begin{array}{c}\mathrm{L}(\mathrm{TIR}) \\
\left(\mathrm{L}_{\odot}\right)\end{array}$ & $\underset{(\mathrm{Jy})}{\operatorname{MIPS~F}(24)^{\mathrm{a}}}$ & $\underset{(\mathrm{Jy})}{\operatorname{IRAS~F}(60)}$ & $\begin{array}{c}\text { IRAS F(100) } \\
\text { (Jy) }\end{array}$ & $\begin{array}{c}\text { Planck F(350) } \\
\text { (Jy) }\end{array}$ & $\underset{(\mathrm{Jy})}{\operatorname{Planck} \mathrm{F}(550)}$ & $\begin{array}{c}\text { Planck (F850) } \\
(\mathrm{Jy})\end{array}$ \\
\hline 219 & NGC 4630 & 9.39 & 0.26 & 2.42 & 5.56 & $2.19 \pm 0.12$ & $\ldots$ & $\ldots$ \\
\hline 220 & IC 3690 & 8.83 & 0.13 & 1.44 & 2.17 & $\ldots$ & $\ldots$ & $\ldots$ \\
\hline 221 & UGC 7905 & 10.45 & 0.15 & 1.82 & 2.53 & $\ldots$ & $\ldots$ & $\ldots$ \\
\hline 222 & MCG 5-30-069 & 10.70 & 0.27 & 3.04 & 5.05 & $\ldots$ & $\ldots$ & $\ldots$ \\
\hline 223 & IC 3721 & 10.85 & 0.18 & 2.02 & 4.14 & $\ldots$ & $\ldots$ & $\ldots$ \\
\hline 224 & NGC 4670 & 9.19 & 0.25 & 2.44 & 4.01 & $\ldots$ & $\ldots$ & $\ldots$ \\
\hline 225 & NGC 4675 & 10.71 & 0.22 & 2.50 & 4.98 & $1.07 \pm 0.14$ & $\ldots$ & $\ldots$ \\
\hline 226 & MCG 7-26-051 & 11.35 & 0.20 & 2.89 & 6.23 & $1.07 \pm 0.19$ & $\ldots$ & $\ldots$ \\
\hline 227 & NGC 4689 & 9.71 & 0.40 & 2.69 & 10.02 & $\ldots$ & $\ldots$ & $\ldots$ \\
\hline 228 & NGC 4688 & 8.97 & 0.12 & 1.00 & 2.05 & $1.71 \pm 0.14$ & $\ldots$ & $\ldots$ \\
\hline 229 & NGC 4704 & 10.96 & 0.33 & 1.70 & 2.32 & $\ldots$ & $\ldots$ & $\ldots$ \\
\hline 230 & NGC 4701 & 9.43 & 0.21 & 2.68 & 6.54 & $2.04 \pm 0.13$ & $\ldots$ & $\ldots$ \\
\hline 231 & IRAS $12468+3436$ & 11.92 & 0.037 & 0.90 & 2.17 & $\ldots$ & $\ldots$ & $\ldots$ \\
\hline 232 & IRAS $12470+1404$ & 8.66 & 0.09 & 0.74 & $<1.36$ & $\ldots$ & $\ldots$ & $\ldots$ \\
\hline 233 & MCG 8-23-097 & 11.36 & 0.29 & 5.08 & 8.10 & $1.37 \pm 0.11$ & $\cdots$ & $\ldots$ \\
\hline 234 & NGC 4747 & 9.24 & $0.30^{\mathrm{b}}$ & 2.16 & 4.29 & $1.86 \pm 0.11$ & $\ldots$ & $\ldots$ \\
\hline 235 & UGC 8017 & 11.01 & 0.17 & 1.90 & 5.22 & $1.18 \pm 0.11$ & $\ldots$ & $\ldots$ \\
\hline 236 & NGC 4765 & 9.24 & 0.17 & 2.23 & 4.18 & $\ldots$ & $\ldots$ & $\ldots$ \\
\hline 237 & VCC 2096 & 8.83 & 0.11 & 1.40 & 2.28 & $\ldots$ & $\ldots$ & $\ldots$ \\
\hline 238 & UGC 8041 & 9.33 & 0.07 & 1.01 & 2.49 & $1.89 \pm 0.11$ & $1.00 \pm 0.11$ & $\ldots$ \\
\hline 239 & UGC 8058 & 12.57 & $8.52^{\mathrm{b}}$ & 33.60 & 30.89 & $1.87 \pm 0.08$ & $\begin{array}{c}1.00 \pm 0.11 \\
\ldots\end{array}$ & $\ldots$ \\
\hline 240 & NGC 4837 & 11.10 & 0.16 & 1.92 & 4.20 & $0.99 \pm 0.13$ & $\ldots$ & $\ldots$ \\
\hline 241 & UM 530 & 11.40 & 0.07 & 0.61 & $<1.78$ & $\ldots$ & $\ldots$ & $\ldots$ \\
\hline 242 & NGC 4861 & 9.32 & 0.34 & 1.98 & 2.38 & $\ldots$ & $\ldots$ & $\ldots$ \\
\hline 243 & NGC 4868 & 10.92 & 0.33 & 3.41 & 8.72 & $2.49 \pm 0.13$ & $0.68 \pm 0.08$ & $\ldots$ \\
\hline 244 & NGC 4922 & 11.34 & 1.16 & 6.61 & 7.08 & $0.90 \pm 0.17$ & $\ldots$ & $\ldots$ \\
\hline 245 & UGC 8179 & 11.25 & 0.07 & 0.92 & 2.18 & $0.89 \pm 0.11$ & $\ldots$ & $\ldots$ \\
\hline 246 & NGC 5001 & 11.28 & 0.22 & 2.67 & 6.19 & $\ldots$ & $\ldots$ & $\ldots$ \\
\hline 247 & IC 856 & 10.40 & 0.13 & 1.62 & 3.56 & $\ldots$ & $\ldots$ & $\ldots$ \\
\hline 248 & UGC 8269 & 11.13 & 0.08 & 2.88 & 5.93 & $2.03 \pm 0.19$ & $0.87 \pm 0.09$ & $\ldots$ \\
\hline 249 & NGC 5014 & 9.44 & $<0.27$ & 2.28 & 4.21 & $0.88 \pm 0.14$ & $\ldots$ & $\ldots$ \\
\hline 250 & NGC 5012 & 10.36 & 0.30 & 2.89 & 8.06 & $3.81 \pm 0.10$ & $\ldots$ & $\ldots$ \\
\hline 251 & IRAS $13116+4508$ & 11.29 & 0.03 & 0.90 & $<1.97$ & $\ldots$ & $\ldots$ & $\ldots$ \\
\hline 252 & IC 860 & 10.95 & 0.89 & 17.60 & 18.70 & $2.05 \pm 0.16$ & $\ldots$ & $\ldots$ \\
\hline 253 & IRAS $13144+4508$ & 11.89 & 0.36 & 0.72 & 1.47 & & $\ldots$ & $\ldots$ \\
\hline 254 & NGC 5060 & 11.06 & 0.29 & 3.06 & 6.87 & $1.52 \pm 0.16$ & $\ldots$ & $\ldots$ \\
\hline 255 & UGC 8357 & 11.25 & 0.23 & 2.52 & 4.62 & $1.42 \pm 0.19$ & $\ldots$ & $\ldots$ \\
\hline 256 & UGC 8361 & 10.95 & 0.23 & 2.79 & 4.46 & $\ldots$ & $\ldots$ & $\ldots$ \\
\hline 257 & IC 883 & 11.66 & 1.06 & 15.10 & 24.38 & $2.55 \pm 0.15$ & $\ldots$ & $\ldots$ \\
\hline 258 & NGC 5100 & 11.21 & 0.17 & 2.12 & 4.68 & $1.43 \pm 0.17$ & $\ldots$ & $\ldots$ \\
\hline 259 & NGC 5104 & 11.23 & 0.56 & 6.99 & 12.74 & $1.57 \pm 0.13$ & $\ldots$ & $\ldots$ \\
\hline 260 & NGC 5107 & 8.97 & 0.06 & 0.88 & 1.65 & & $\ldots$ & $\ldots$ \\
\hline 261 & NGC 5112 & 9.53 & 0.16 & 2.46 & 6.06 & $2.25 \pm 0.16$ & $0.63 \pm 0.10$ & $\ldots$ \\
\hline 262 & NGC 5123 & 11.14 & 0.17 & 1.68 & 5.28 & $1.77 \pm 0.12$ & $\ldots$ & $\ldots$ \\
\hline 263 & IRAS $13218+0552$ & 12.37 & 0.23 & 1.25 & $<1.00$ & & $\ldots$ & $\ldots$ \\
\hline 264 & IRAS $13232+1731$ & 11.59 & 0.07 & 0.66 & 2.02 & $1.23 \pm 0.18$ & $\ldots$ & $\ldots$ \\
\hline 265 & NGC 5147 & 9.57 & 0.23 & 2.88 & 6.74 & $2.62 \pm 0.13$ & $0.90 \pm 0.14$ & $\ldots$ \\
\hline 266 & NGC 5204 & 7.95 & 0.18 & 2.86 & 5.02 & $2.50 \pm 0.13$ & $1.05 \pm 0.07$ & $0.32 \pm 0.05$ \\
\hline 267 & UGC 8502 & 11.09 & 0.19 & 1.61 & 2.80 & $\cdots$ & $\ldots$ & $\cdots$ \\
\hline 268 & UGC 8561 & 11.14 & 0.25 & 2.78 & 6.92 & $\ldots$ & $\ldots$ & $\ldots$ \\
\hline 259 & NGC 5230 & 10.99 & 0.19 & 1.56 & 4.95 & $1.50 \pm 0.18$ & $\ldots$ & $\ldots$ \\
\hline 270 & IRAS $13349+2438$ & 12.29 & 0.87 & 0.69 & $<1.00$ & $\ldots$ & $\ldots$ & $\ldots$ \\
\hline 271 & NGC 5256 & 11.58 & 0.86 & 7.29 & 12.25 & $\ldots$ & $\ldots$ & $\ldots$ \\
\hline 272 & UGC 8626 & 10.66 & 0.11 & 1.41 & 2.18 & $\ldots$ & $\ldots$ & $\ldots$ \\
\hline 273 & NGC 5263 & 10.88 & 0.30 & 3.65 & 7.40 & $1.88 \pm 0.09$ & $\ldots$ & $\ldots$ \\
\hline 274 & MCG 1-35-028 & 10.98 & 0.35 & 2.71 & 4.17 & $\cdots$ & $\ldots$ & $\cdots$ \\
\hline 275 & IC 910 & 11.24 & 0.47 & 5.05 & 6.28 & $\ldots$ & $\ldots$ & $\ldots$ \\
\hline 276 & MK 268 & 11.13 & 0.24 & 1.43 & 1.79 & $\ldots$ & $\ldots$ & $\ldots$ \\
\hline 277 & NGC 5278 & 10.97 & 0.11 & 1.51 & 4.39 & $1.46 \pm 0.13$ & $\ldots$ & $\ldots$ \\
\hline 278 & NGC 5273 & 8.81 & 0.10 & 0.81 & 1.13 & $\ldots$ & $\ldots$ & $\ldots$ \\
\hline 279 & UGC 8685 & 11.31 & 0.18 & 2.30 & 5.17 & $0.90 \pm 0.12$ & $\ldots$ & $\ldots$ \\
\hline 280 & UGC 8686 & 10.94 & 0.18 & 1.88 & 4.45 & $1.13 \pm 0.13$ & $\ldots$ & $\cdots$ \\
\hline 281 & UGC 8696 & 12.10 & $2.33^{\mathrm{b}}$ & 23.70 & 22.31 & $1.56 \pm 0.12$ & $\ldots$ & $\ldots$ \\
\hline 282 & NGC 5297 & 10.08 & 0.23 & 2.43 & 7.40 & $3.62 \pm 0.14$ & $1.13 \pm 0.09$ & $0.45 \pm 0.07$ \\
\hline 283 & MK 796 & 11.09 & 0.58 & 3.17 & 5.44 & $\cdots$ & $\ldots$ & $\cdots$ \\
\hline 284 & IRAS $13446+1121$ & 11.02 & 0.57 & 3.43 & 3.59 & $\ldots$ & $\ldots$ & $\ldots$ \\
\hline 285 & NGC 5303 & 9.78 & 0.28 & 3.09 & 6.50 & $\ldots$ & $\ldots$ & $\ldots$ \\
\hline 286 & NGC 5313 & 10.24 & 0.33 & 3.09 & 10.53 & $3.11 \pm 0.13$ & $0.93 \pm 0.09$ & $\ldots$ \\
\hline 287 & MCG 3-35-034 & 11.16 & 0.08 & 1.20 & 2.80 & & $\ldots$ & $\ldots$ \\
\hline 288 & NGC 5347 & 10.24 & 0.84 & 1.44 & 2.71 & $1.18 \pm 0.17$ & $\ldots$ & $\ldots$ \\
\hline 289 & NGC 5350 & 10.16 & 0.39 & 2.72 & 8.11 & $\ldots$ & $\cdots$ & $\ldots$ \\
\hline 290 & NGC 5368 & 10.43 & 0.12 & 1.32 & 2.74 & $\ldots$ & $\ldots$ & $\ldots$ \\
\hline 291 & UGC 8827 & 10.94 & 0.49 & 3.90 & 5.82 & $\ldots$ & $\ldots$ & $\ldots$ \\
\hline 292 & UGC 8850 & 11.85 & 1.39 & 2.18 & 1.87 & $\ldots$ & $\ldots$ & $\ldots$ \\
\hline
\end{tabular}


Ashby et al.

TABLE 7 Continued

\begin{tabular}{|c|c|c|c|c|c|c|c|c|}
\hline SFRS & Name & $\begin{array}{c}\mathrm{L}(\mathrm{TIR}) \\
\left(\mathrm{L}_{\odot}\right)\end{array}$ & $\begin{array}{c}\operatorname{MIPS~F}(24)^{\mathrm{a}} \\
(\mathrm{Jy})\end{array}$ & $\begin{array}{c}\text { IRAS F(60) } \\
(\mathrm{Jy})\end{array}$ & $\begin{array}{c}\text { IRAS F(100) } \\
(\mathrm{Jy})\end{array}$ & $\begin{array}{c}\text { Planck F(350) } \\
\text { (Jy) }\end{array}$ & $\underset{(J y)}{\operatorname{Planck~F}(550)}$ & $\underset{(\mathrm{Jy})}{\text { Planck (F850) }}$ \\
\hline 293 & UGC 8856 & 11.14 & 0.14 & 2.02 & 4.35 & . & $\ldots$ & $\ldots$ \\
\hline 294 & NGC 5374 & 10.77 & 0.29 & 2.81 & 6.99 & $1.34 \pm 0.16$ & $\ldots$ & $\ldots$ \\
\hline 295 & UGC 8902 & 10.86 & 0.11 & 1.26 & 3.26 & $1.13 \pm 0.14$ & $\ldots$ & $\ldots$ \\
\hline 296 & NGC 5403 & 10.36 & 0.18 & 2.88 & 10.60 & $3.95 \pm 0.15$ & $1.03 \pm 0.10$ & $\ldots$ \\
\hline 297 & MCG 7-29-036 & 11.19 & 0.19 & 2.47 & 4.25 & $1.14 \pm 0.11$ & $\ldots$ & $\ldots$ \\
\hline 298 & NGC 5414 & 10.56 & 0.24 & 2.55 & 4.23 & $\ldots$ & $\ldots$ & $\ldots$ \\
\hline 299 & MCG 5-33-046 & 11.02 & 0.26 & 2.71 & 4.16 & $\ldots$ & $\ldots$ & $\ldots$ \\
\hline 300 & NGC 5474 & 8.44 & 0.14 & 2.22 & 5.45 & $3.01 \pm 0.20$ & $1.31 \pm 0.08$ & $\ldots$ \\
\hline 301 & NGC 5480 & 10.21 & 0.37 & 3.66 & 10.13 & $2.85 \pm 0.18$ & $0.86 \pm 0.10$ & $\ldots$ \\
\hline 302 & MCG 6-31-070 & 11.31 & 0.21 & 2.61 & 4.89 & $0.86 \pm 0.10$ & ... & $\ldots$ \\
\hline 303 & CGCG 74-129 & 10.81 & 0.83 & 3.69 & 2.98 & $\ldots$ & $\ldots$ & $\ldots$ \\
\hline 304 & NGC 5520 & 9.95 & 0.19 & 2.31 & 5.75 & $1.39 \pm 0.11$ & $\ldots$ & $\ldots$ \\
\hline 305 & NGC 5515 & 11.02 & 0.16 & 1.79 & 4.78 & $\ldots$ & $\ldots$ & $\ldots$ \\
\hline 306 & NGC 5526 & 9.82 & 0.17 & 2.16 & 5.04 & $1.69 \pm 0.14$ & $\ldots$ & $\ldots$ \\
\hline 307 & NGC 5522 & 10.57 & 0.15 & 2.06 & 4.28 & $1.80 \pm 0.12$ & $\ldots$ & $\ldots$ \\
\hline 308 & NGC 5541 & 11.09 & 0.22 & 2.39 & 5.34 & $1.60 \pm 0.21$ & $\ldots$ & $\ldots$ \\
\hline 309 & IC 4395 & 11.39 & 0.22 & 2.87 & 5.59 & $\begin{array}{c}1.000 .21 \\
\ldots\end{array}$ & $\ldots$ & $\ldots$ \\
\hline 310 & UGC 9165 & 10.90 & 0.23 & 3.10 & 7.18 & $1.41 \pm 0.14$ & $\ldots$ & $\ldots$ \\
\hline 311 & MK 1490 & 11.36 & 0.70 & 6.21 & 8.39 & $1.63 \pm 0.18$ & $\ldots$ & $\ldots$ \\
\hline 312 & NGC 5585 & 8.35 & 0.12 & 1.70 & 4.40 & $2.63 \pm 0.12$ & $0.96 \pm 0.09$ & $\ldots$ \\
\hline 313 & IC 4408 & 11.17 & 0.17 & 1.83 & 4.67 & $1.16 \pm 0.10$ & & $\ldots$ \\
\hline 314 & NGC 5584 & 9.77 & 0.28 & 2.56 & 6.11 & $3.31 \pm 0.17$ & $1.04 \pm 0.12$ & $\ldots$ \\
\hline 315 & NGC 5633 & 10.26 & 0.27 & 2.67 & 7.84 & $1.76 \pm 0.09$ & $\ldots$ & $\ldots$ \\
\hline 316 & NGC 5660 & 10.37 & 0.34 & 4.14 & 9.16 & $3.07 \pm 0.18$ & $0.96 \pm 0.11$ & $\ldots$ \\
\hline 317 & NGC 5656 & 10.22 & 0.24 & 1.54 & 2.66 & $\ldots$ & $\ldots$ & $\ldots$ \\
\hline 318 & NGC 5657 & 10.76 & $0.29^{\mathrm{b}}$ & 2.70 & 7.92 & $2.72 \pm 0.15$ & $1.00 \pm 0.09$ & $\ldots$ \\
\hline 319 & CGCG 133-083 & 11.41 & 0.19 & 2.25 & 4.03 & & $\ldots$ & $\ldots$ \\
\hline 320 & MCG 7-30-028 & 11.04 & 0.14 & 1.82 & 4.88 & $0.99 \pm 0.10$ & $\ldots$ & $\ldots$ \\
\hline 321 & MCG 6-32-070 & 11.21 & 0.25 & 2.86 & 5.81 & $1.00 \pm 0.11$ & $\ldots$ & $\ldots$ \\
\hline 322 & UGC 9412 & 11.34 & 0.92 & 2.24 & 2.28 & & $\ldots$ & $\ldots$ \\
\hline 323 & NGC 5698 & 10.33 & 0.24 & 1.27 & 2.61 & $0.82 \pm 0.10$ & $\ldots$ & $\ldots$ \\
\hline 324 & NGC 5691 & 9.65 & 0.28 & 3.43 & 6.60 & $\ldots$ & $\ldots$ & $\ldots$ \\
\hline 325 & MCG 9-24-035 & 11.18 & 0.32 & 3.13 & 4.07 & $\ldots$ & $\ldots$ & $\ldots$ \\
\hline 326 & MCG 9-24-038 & 11.17 & 0.10 & 1.34 & 3.21 & $\ldots$ & $\ldots$ & $\ldots$ \\
\hline 327 & UGC 9560 & 9.19 & 0.10 & 0.71 & 1.46 & $\ldots$ & $\ldots$ & $\ldots$ \\
\hline 328 & IC 1076 & 10.73 & 0.15 & 1.66 & 3.56 & $\ldots$ & $\ldots$ & $\ldots$ \\
\hline 329 & IRAS $14538+1730$ & 11.93 & 0.08 & 1.39 & 2.84 & $\ldots$ & $\ldots$ & $\ldots$ \\
\hline 330 & NGC 5795 & 10.23 & 0.22 & 2.65 & 6.99 & $1.43 \pm 0.12$ & $\ldots$ & $\ldots$ \\
\hline 331 & UGC 9618 & 11.73 & 0.40 & 6.45 & 15.52 & $3.08 \pm 0.17$ & $0.84 \pm 0.10$ & $\ldots$ \\
\hline 332 & UGC 9639 & 11.40 & 0.32 & 2.47 & 5.23 & $1.19 \pm 0.14$ & $\ldots$ & $\ldots$ \\
\hline 333 & MCG 6-33-022 & 11.50 & 0.15 & 2.58 & 5.25 & $1.05 \pm 0.12$ & $\ldots$ & $\ldots$ \\
\hline 334 & NGC 5879 & 9.58 & 0.33 & 3.30 & 9.11 & $\ldots$ & $\ldots$ & $0.28 \pm 0.04$ \\
\hline 335 & MCG 9-25-036 & 10.86 & 0.013 & 0.65 & 1.91 & $\ldots$ & $\ldots$ & $\ldots$ \\
\hline 336 & NGC 5899 & 10.61 & 0.55 & 3.20 & 11.53 & $4.59 \pm 0.23$ & $1.28 \pm 0.11$ & \\
\hline 337 & NGC 5905 & 10.63 & 0.33 & 2.82 & 6.73 & $2.78 \pm 0.10$ & $0.82 \pm 0.07$ & $0.26 \pm 0.04$ \\
\hline 338 & MK 848 & 11.85 & 1.19 & 9.39 & 10.26 & & $\ldots$ & \\
\hline 339 & IC 4553 & 12.12 & 5.42 & 104.10 & 117.70 & $13.94 \pm 0.20$ & $3.64 \pm 0.12$ & $0.94 \pm 0.08$ \\
\hline 340 & UGC 9922 & 10.76 & 0.20 & 2.31 & 4.30 & $\ldots$ & $\ldots$ & $\ldots$ \\
\hline 341 & IC 4567 & 10.93 & 0.21 & 2.18 & 6.29 & $1.73 \pm 0.20$ & $\ldots$ & $\ldots$ \\
\hline 342 & MCG 4-37-016 & 10.91 & 0.45 & 2.27 & 2.88 & $\cdots$ & $\ldots$ & $\ldots$ \\
\hline 343 & NGC 5975 & 10.77 & 0.29 & 3.86 & 7.23 & $\ldots$ & $\ldots$ & $\ldots$ \\
\hline 344 & NGC 5980 & 10.80 & 0.32 & 3.33 & 8.60 & $2.31 \pm 0.11$ & $\ldots$ & $\ldots$ \\
\hline 345 & NGC 5992 & 11.19 & 0.21 & 1.50 & $<4.27$ & $\cdots$ & $\ldots$ & $\ldots$ \\
\hline 346 & NGC 5996 & 10.63 & 0.47 & 4.10 & 7.60 & $\ldots$ & $\ldots$ & $\ldots$ \\
\hline 347 & IRAS $15519+3537$ & 11.60 & 0.049 & 0.88 & 1.99 & $\ldots$ & $\ldots$ & $\ldots$ \\
\hline 348 & UGC 10099 & 11.15 & 0.16 & 1.86 & 3.45 & $\ldots$ & $\ldots$ & $\ldots$ \\
\hline 349 & MCG 5-38-006 & 10.64 & 0.58 & 3.06 & 3.07 & $\ldots$ & $\ldots$ & $\ldots$ \\
\hline 350 & UGC 10120 & 10.79 & 0.15 & 0.64 & 1.34 & $\ldots$ & $\ldots$ & $\ldots$ \\
\hline 351 & NGC 6027A & 10.21 & 0.033 & 1.03 & 2.20 & $\ldots$ & $\ldots$ & $\ldots$ \\
\hline 352 & NGC 6039 & 11.11 & $<0.25$ & 0.77 & 1.34 & $\ldots$ & $\ldots$ & $\ldots$ \\
\hline 353 & UGC 10200 & 9.62 & 0.21 & 1.46 & 1.86 & $\ldots$ & $\ldots$ & $\ldots$ \\
\hline 354 & IRAS $16052+5334$ & 11.70 & 0.044 & 0.80 & 2.37 & $\ldots$ & $\ldots$ & $\ldots$ \\
\hline 355 & IRAS $16053+1836$ & 11.19 & 0.18 & 2.11 & 3.32 & $\ldots$ & $\ldots$ & $\ldots$ \\
\hline 356 & NGC 6090 & 11.57 & 0.93 & 6.49 & 9.98 & $\ldots$ & $\ldots$ & $\ldots$ \\
\hline 357 & UGC 10273 & 10.97 & 0.16 & 1.77 & 4.36 & $2.26 \pm 0.26$ & $\ldots$ & $\ldots$ \\
\hline 358 & IRAS $16150+2233$ & 11.76 & 0.12 & 0.61 & $4.28^{\mathrm{d}}$ & $\ldots$ & $\ldots$ & $\ldots$ \\
\hline 359 & UGC 10322 & 10.66 & 0.19 & 1.96 & 5.58 & $\ldots$ & $\ldots$ & $\ldots$ \\
\hline 360 & NGC 6120 & 11.39 & 0.27 & 4.01 & 8.26 & $1.31 \pm 0.18$ & $\ldots$ & $\ldots$ \\
\hline 361 & MCG 3-42-004 & 11.18 & 0.12 & 1.34 & 2.94 & $\ldots$ & $\ldots$ & $\ldots$ \\
\hline 362 & UGC 10407 & 10.93 & 0.15 & 1.74 & 3.13 & $\ldots$ & $\ldots$ & $\ldots$ \\
\hline 363 & IRAS $16320+3922$ & 10.75 & 0.014 & 0.67 & 1.98 & $\ldots$ & $\ldots$ & $\ldots$ \\
\hline 364 & NGC 6186 & 11.46 & 0.26 & 2.92 & 6.27 & $\ldots$ & $\ldots$ & $\ldots$ \\
\hline 365 & MCG 9-27-053 & 11.10 & 0.07 & 2.22 & 5.10 & $\ldots$ & $\ldots$ & $\ldots$ \\
\hline 366 & UGC 10514 & 10.90 & 0.22 & 2.65 & 4.47 & $\ldots$ & $\ldots$ & $\ldots$ \\
\hline
\end{tabular}


TABLE $7-$ Continued

\begin{tabular}{|c|c|c|c|c|c|c|c|c|}
\hline SFRS & Name & $\begin{array}{c}\mathrm{L}(\mathrm{TIR}) \\
\left(\mathrm{L}_{\odot}\right)\end{array}$ & $\underset{(\mathrm{Jy})}{\operatorname{MIPS} \mathrm{F}(24)^{\mathrm{a}}}$ & $\underset{(\mathrm{Jy})}{\operatorname{IRAS~F}(60)}$ & $\begin{array}{c}\text { IRAS F(100) } \\
(\mathrm{Jy})\end{array}$ & $\underset{\text { (Jy) }}{\text { Planck F }}$ & $\underset{(\mathrm{Jy})}{\operatorname{Planck} \mathrm{F}(550)}$ & $\begin{array}{c}\text { Planck (F850) } \\
(\text { Jy) }\end{array}$ \\
\hline 367 & IRAS $16435+2154$ & 11.09 & 0.11 & 2.07 & 3.81 & $\cdots$ & $\cdots$ & $\cdots$ \\
\hline 368 & IC 4623 & 11.07 & 0.14 & 1.62 & 3.53 & $\ldots$ & $\ldots$ & $\ldots$ \\
\hline 369 & IRAS $16516+3030$ & 11.71 & 0.12 & 1.63 & 3.21 & $\ldots$ & $\ldots$ & $\ldots$ \\
\hline
\end{tabular}

Note. -

a The MIPS $24 \mu \mathrm{m}$ photometry has been aperture corrected using the correction factors listed in Table 8 . The uncertainties in total $24 \mu \mathrm{m}$ flux densities are dominated by the uncertainty in the absolute calibration, which is estimated as 4-8\% according to the MIPS Instrument Handbook, version 2. Where upper limits are given, the photometry is from IRAS with quality flag $=1$.

b IRAS flux density (MIPS not available). Typical uncertainties are 10-20\%.

c OJ 287 is highly variable. Flux densities here are from IRAS survey coadds (Impey \& Neugebauer 1988), but many more measurements exist in the literature.

d IRAS flux densities taken from Rice et al. 1988.

e Measurement taken from Dale et al. 2005. 
Ashby et al.

TABLE 8

Spitzer/Mips Aperture Correction Factors for SFRS Galaxies

\begin{tabular}{|c|c|c|}
\hline SFRS & Name & Factor \\
\hline 24 & NGC 2623 & 1.01 \\
\hline 35 & NGC 2719 & 1.01 \\
\hline 36 & IRAS $08572+3915$ & 1.04 \\
\hline 52 & NGC 2854 & 1.05 \\
\hline 60 & MCG 8-18-013 & 1.05 \\
\hline 70 & NGC 3049 & 1.01 \\
\hline 85 & UGC 5644 & 1.07 \\
\hline 90 & NGC 3265 & 1.03 \\
\hline 93 & UGC 5720 & 1.03 \\
\hline 104 & NGC 3413 & 1.07 \\
\hline 106 & CGCG 95-055 & 1.08 \\
\hline 108 & IRAS $10565+2448$ & 1.05 \\
\hline 112 & MCG 7-23-019 & 1.07 \\
\hline 115 & IRAS $11069+2711$ & 1.02 \\
\hline 116 & IC 676 & 1.06 \\
\hline 117 & IRAS $11102+3026$ & 1.08 \\
\hline 118 & IC 2637 & 1.08 \\
\hline 120 & 7ZW 384 & 1.06 \\
\hline 121 & IRAS $11167+5351$ & 1.07 \\
\hline 124 & NGC 3656 & 1.11 \\
\hline 125 & NGC 3659 & 1.02 \\
\hline 128 & IC 691 & 1.06 \\
\hline 130 & UGC 6469 & 1.05 \\
\hline 132 & IC 698 & 1.06 \\
\hline 133 & IRAS $11267+1558$ & 1.12 \\
\hline 135 & MCG 3-29-061 & 1.07 \\
\hline 136 & NGC 3720 & 1.08 \\
\hline 138 & MCG 10-17-019 & 1.07 \\
\hline 139 & NGC 3758 & 1.07 \\
\hline 143 & NGC 3773 & 1.05 \\
\hline 144 & NGC 3781 & 1.05 \\
\hline 145 & UGC 6625 & 1.05 \\
\hline 146 & NGC 3808A & 1.09 \\
\hline 148 & NGC 3822 & 1.06 \\
\hline 150 & MCG 3-30-051 & 1.06 \\
\hline 151 & NGC 3839 & 1.04 \\
\hline 152 & UGC 6732 & 1.05 \\
\hline 153 & IC 730 & 1.08 \\
\hline 155 & NGC 3912 & 1.03 \\
\hline 156 & NGC 3928 & 1.05 \\
\hline 157 & NGC 3934 & 1.05 \\
\hline 158 & UGC 6865 & 1.01 \\
\hline 159 & UGC 6901 & 1.04 \\
\hline 162 & NGC 4004 & 1.02 \\
\hline 163 & NGC 4014 & 1.03 \\
\hline 165 & NGC 4018 & 1.03 \\
\hline 167 & IRAS $11571+3003$ & 1.07 \\
\hline 168 & UGC 7017 & 1.05 \\
\hline 169 & UGC 7016 & 1.04 \\
\hline 170 & MCG 3-31-030 & 1.08 \\
\hline 176 & NGC 4150 & 1.07 \\
\hline 180 & IRAS $12112+0305$ & 1.02 \\
\hline 184 & NGC 4207 & 1.01 \\
\hline 185 & UGC 7286 & 1.09 \\
\hline 186 & NGC 4234 & 1.04 \\
\hline 190 & MCG 3-32-005 & 1.07 \\
\hline 191 & NGC 4290 & 1.02 \\
\hline 193 & NGC 4314 & 1.04 \\
\hline 194 & NGC 4385 & 1.05 \\
\hline 197 & NGC 4412 & 1.04 \\
\hline 206 & IRAS $12265+0219$ & 1.07 \\
\hline 206 & IRAS $12274+0018$ & 1.11 \\
\hline 207 & NGC 4491 & 1.04 \\
\hline 208 & NGC 4500 & 1.04 \\
\hline 209 & NGC 4495 & 1.06 \\
\hline 211 & NGC 4509 & 1.08 \\
\hline 214 & IRAS $12337+5044$ & 1.06 \\
\hline 215 & IC 3581 & 1.05 \\
\hline 219 & NGC 4630 & 1.02 \\
\hline 221 & UGC 7905 & 1.02 \\
\hline 222 & MCG 5-30-069 & 1.07 \\
\hline 223 & IC 3721 & 1.08 \\
\hline 224 & NGC 4670 & 1.05 \\
\hline 229 & NGC 4704 & 1.04 \\
\hline
\end{tabular}


TABLE 8 - Continued

\begin{tabular}{|c|c|c|}
\hline SFRS & Name & Factor \\
\hline 230 & NGC 4701 & 1.01 \\
\hline 231 & IRAS $12468+3436$ & 1.05 \\
\hline 232 & IRAS $12470+1404$ & 1.08 \\
\hline 233 & MCG 8-23-097 & 1.06 \\
\hline 235 & UGC 8017 & 1.04 \\
\hline 236 & NGC 4765 & 1.05 \\
\hline 237 & VCC2096 & 1.01 \\
\hline 239 & UGC 8058 & 1.02 \\
\hline 240 & NGC 4837 & 1.05 \\
\hline 241 & UM 530 & 1.10 \\
\hline 242 & NGC 4861 & 1.01 \\
\hline 243 & NGC 4868 & 1.04 \\
\hline 244 & NGC 4922 & 1.05 \\
\hline 245 & UGC 8179 & 1.05 \\
\hline 246 & NGC 5001 & 1.06 \\
\hline 247 & IC 856 & 1.07 \\
\hline 248 & UGC 8269 & 1.09 \\
\hline 251 & IRAS $13116+4508$ & 1.10 \\
\hline 252 & IC 860 & 1.05 \\
\hline 253 & IRAS $13144+4508$ & 1.04 \\
\hline 254 & NGC 5060 & 1.06 \\
\hline 255 & UGC 8357 & 1.07 \\
\hline 256 & UGC 8361 & 1.08 \\
\hline 257 & IC 883 & 1.06 \\
\hline 258 & NGC 5100 & 1.04 \\
\hline 259 & NGC 5104 & 1.07 \\
\hline 262 & NGC 5123 & 1.03 \\
\hline 263 & IRAS $13218+0552$ & 1.08 \\
\hline 264 & IRAS $13232+1731$ & 1.06 \\
\hline 267 & UGC 8502 & 1.06 \\
\hline 268 & UGC 8561 & 1.05 \\
\hline 271 & NGC 5256 & 1.02 \\
\hline 272 & UGC 8626 & 1.08 \\
\hline 273 & NGC 5263 & 1.03 \\
\hline 274 & MCG 1-35-028 & 1.05 \\
\hline 275 & IC 910 & 1.05 \\
\hline 276 & MK 268 & 1.05 \\
\hline 277 & NGC 5278 & 1.05 \\
\hline 278 & NGC 5273 & 1.07 \\
\hline 279 & UGC 8685 & 1.06 \\
\hline 283 & MK 796 & 1.02 \\
\hline 284 & IRAS $13446+1121$ & 1.04 \\
\hline 285 & NGC 5303 & 1.04 \\
\hline 287 & MCG 3-35-034 & 1.07 \\
\hline 290 & NGC 5368 & 1.04 \\
\hline 291 & UGC 8827 & 1.04 \\
\hline 293 & UGC 8856 & 1.08 \\
\hline 294 & NGC 5374 & 1.03 \\
\hline 295 & UGC 8902 & 1.03 \\
\hline 298 & NGC 5414 & 1.07 \\
\hline 299 & MCG 5-33-046 & 1.06 \\
\hline 301 & NGC 5480 & 1.01 \\
\hline 302 & MCG 6-31-070 & 1.07 \\
\hline 303 & CGCG 74-129 & 1.05 \\
\hline 304 & NGC 5520 & 1.03 \\
\hline 305 & NGC 5515 & 1.06 \\
\hline 306 & NGC 5526 & 1.02 \\
\hline 307 & NGC 5522 & 1.04 \\
\hline 308 & NGC 5541 & 1.05 \\
\hline 309 & IC 4395 & 1.06 \\
\hline 310 & UGC 9165 & 1.04 \\
\hline 311 & MK 1490 & 1.06 \\
\hline 312 & IC 4408 & 1.01 \\
\hline 315 & NGC 5633 & 1.03 \\
\hline 317 & NGC 5656 & 1.02 \\
\hline 319 & CGCG 133-083 & 1.08 \\
\hline 321 & MCG 6-32-070 & 1.05 \\
\hline 322 & UGC 9412 & 1.04 \\
\hline 323 & NGC 5698 & 1.04 \\
\hline 324 & NGC 5691 & 1.03 \\
\hline 325 & MCG 9-24-035 & 1.07 \\
\hline 326 & MCG 9-24-038 & 1.06 \\
\hline 327 & UGC 9560 & 1.07 \\
\hline 328 & IC 1076 & 1.05 \\
\hline 329 & IRAS $14538+1730$ & 1.11 \\
\hline 330 & NGC 5795 & 1.03 \\
\hline
\end{tabular}


Ashby et al.

TABLE 8 - Continued

\begin{tabular}{rrr}
\hline \hline SFRS & \multicolumn{1}{c}{ Name } & Factor \\
& & \\
\hline 332 & UGC 9639 & 1.05 \\
333 & MCG 6-33-022 & 1.05 \\
335 & MCG 9-25-036 & 1.10 \\
338 & MK 848 & 1.02 \\
340 & UGC 9922 & 1.05 \\
341 & IC 4567 & 1.03 \\
342 & MCG 4-37-016 & 1.03 \\
343 & NGC 5975 & 1.06 \\
344 & NGC 5980 & 1.01 \\
345 & NGC 5992 & 1.07 \\
347 & IRAS 15519+3537 & 1.10 \\
348 & UGC 10099 & 1.06 \\
349 & MCG 5-38-006 & 1.05 \\
350 & UGC 10120 & 1.08 \\
351 & NGC6027A & 1.03 \\
353 & UGC 10200 & 1.03 \\
355 & IRAS 16053+1836 & 1.07 \\
356 & NGC 6090 & 1.02 \\
357 & UGC 10273 & 1.01 \\
358 & IRAS 16150+2233 & 1.10 \\
359 & UGC 10322 & 1.05 \\
360 & NGC 6120 & 1.05 \\
361 & MCG 3-42-004 & 1.08 \\
362 & UGC 10407 & 1.04 \\
363 & IRAS 16320+3922 & 1.10 \\
364 & NGC 6186 & 1.03 \\
365 & MCG 9-27-053 & 1.08 \\
366 & UGC 10514 & 1.06 \\
367 & IRAS 16435+2154 & 1.08 \\
368 & IC 4623 & 1.02 \\
369 & IRAS 16516+3030 & 1.06 \\
& & \\
& &
\end{tabular}

Note. - MIPS $24 \mu \mathrm{m}$ aperture correction factors based on Version 2 of the MIPS Instrument Handbook, page 94 . These factors have been applied to the photometry presented in Table 7 . When not given, the correction factor was taken to be unity. 
TABLE 9

Visible and Near-Infrared Photometry for SFRS Galaxies

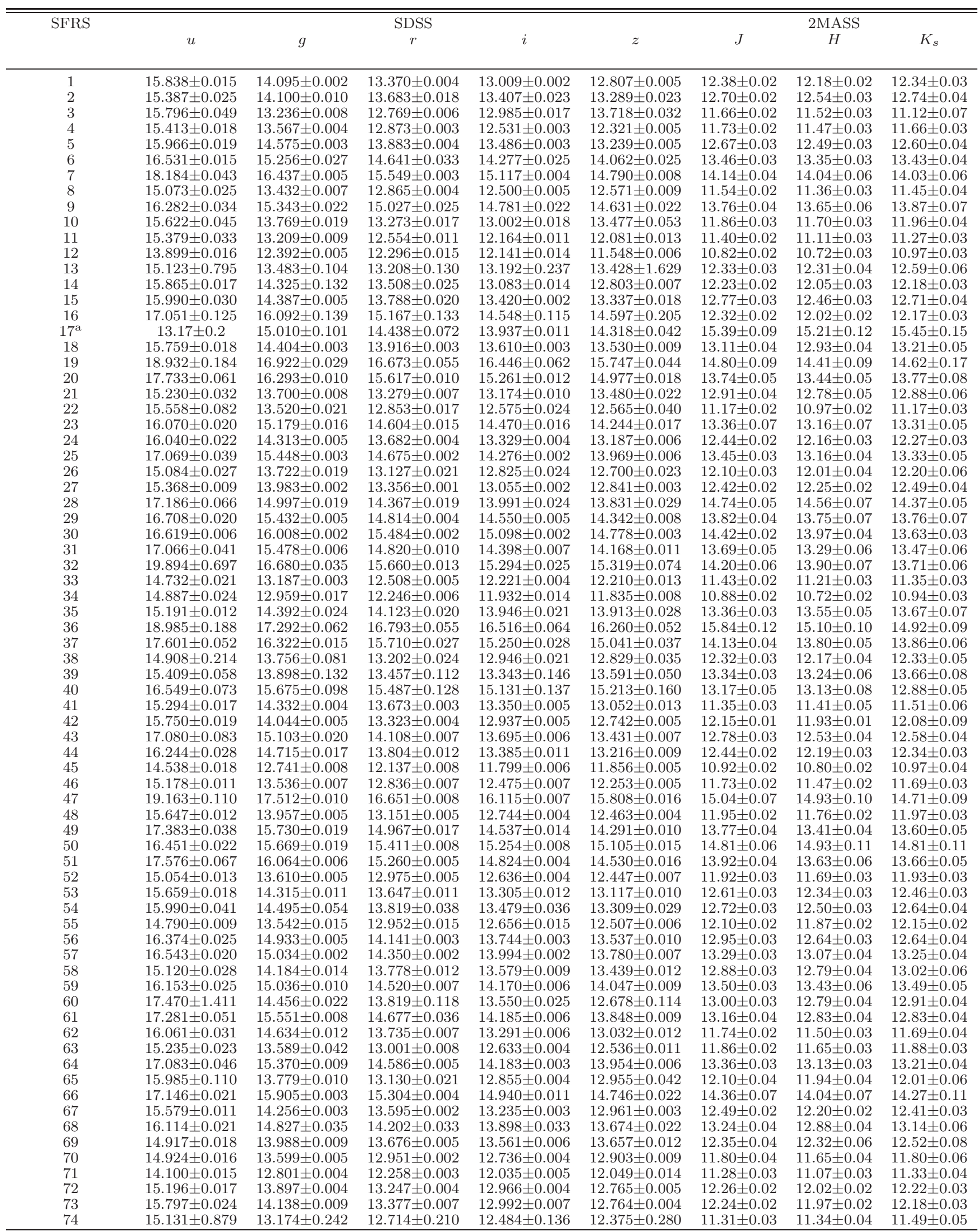


TABLE 9 - Continued

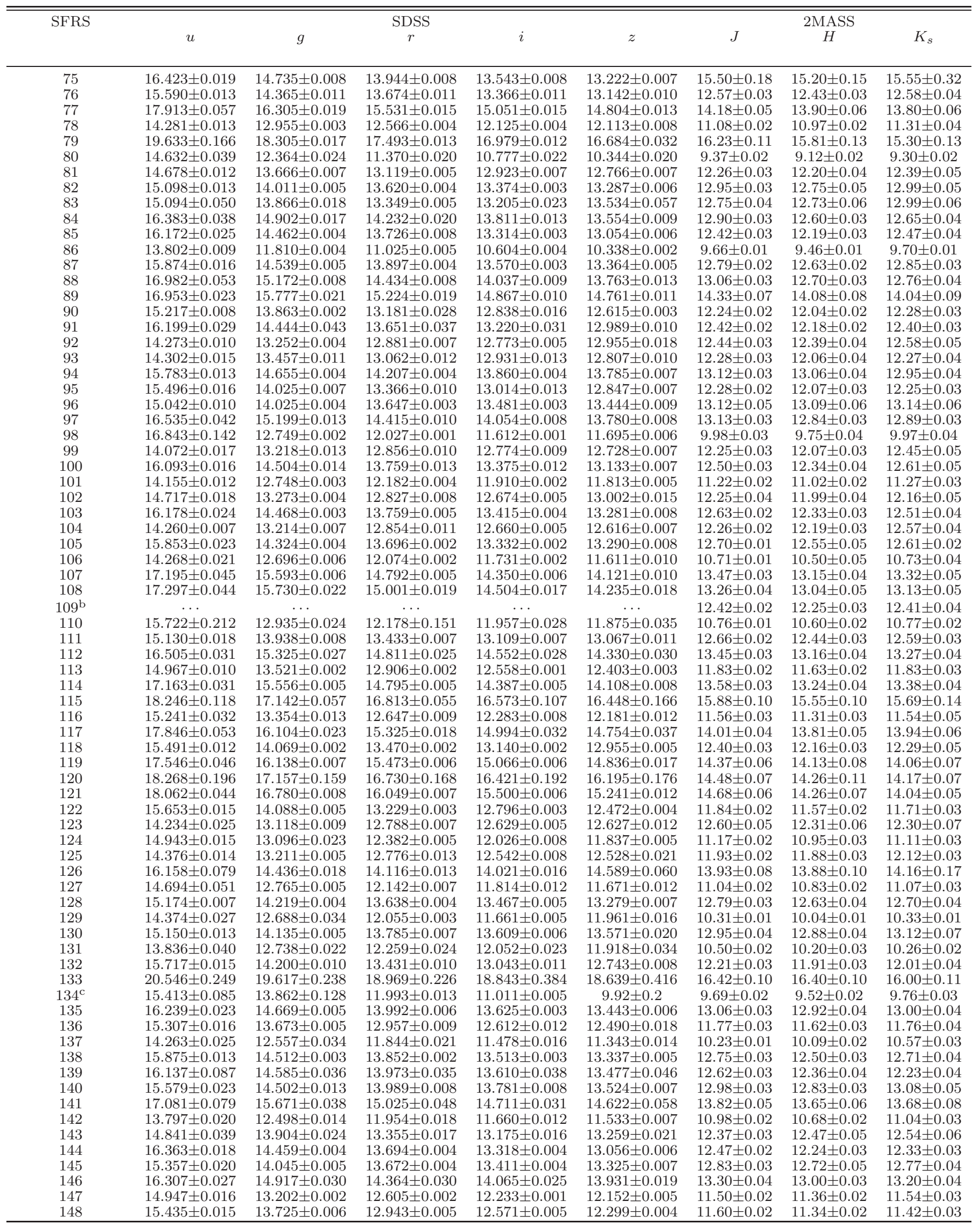


TABLE $9-$ Continued

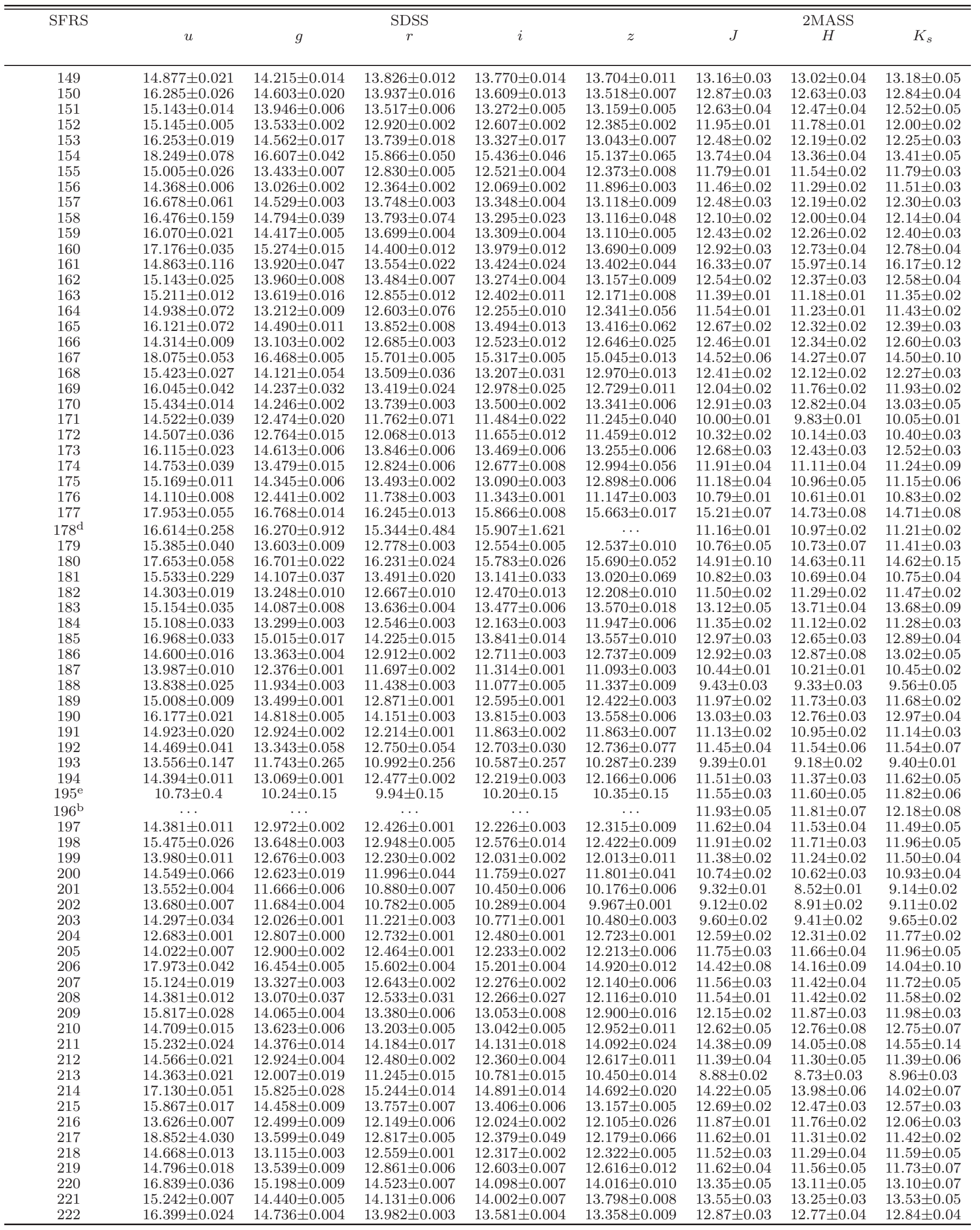


TABLE 9 - Continued

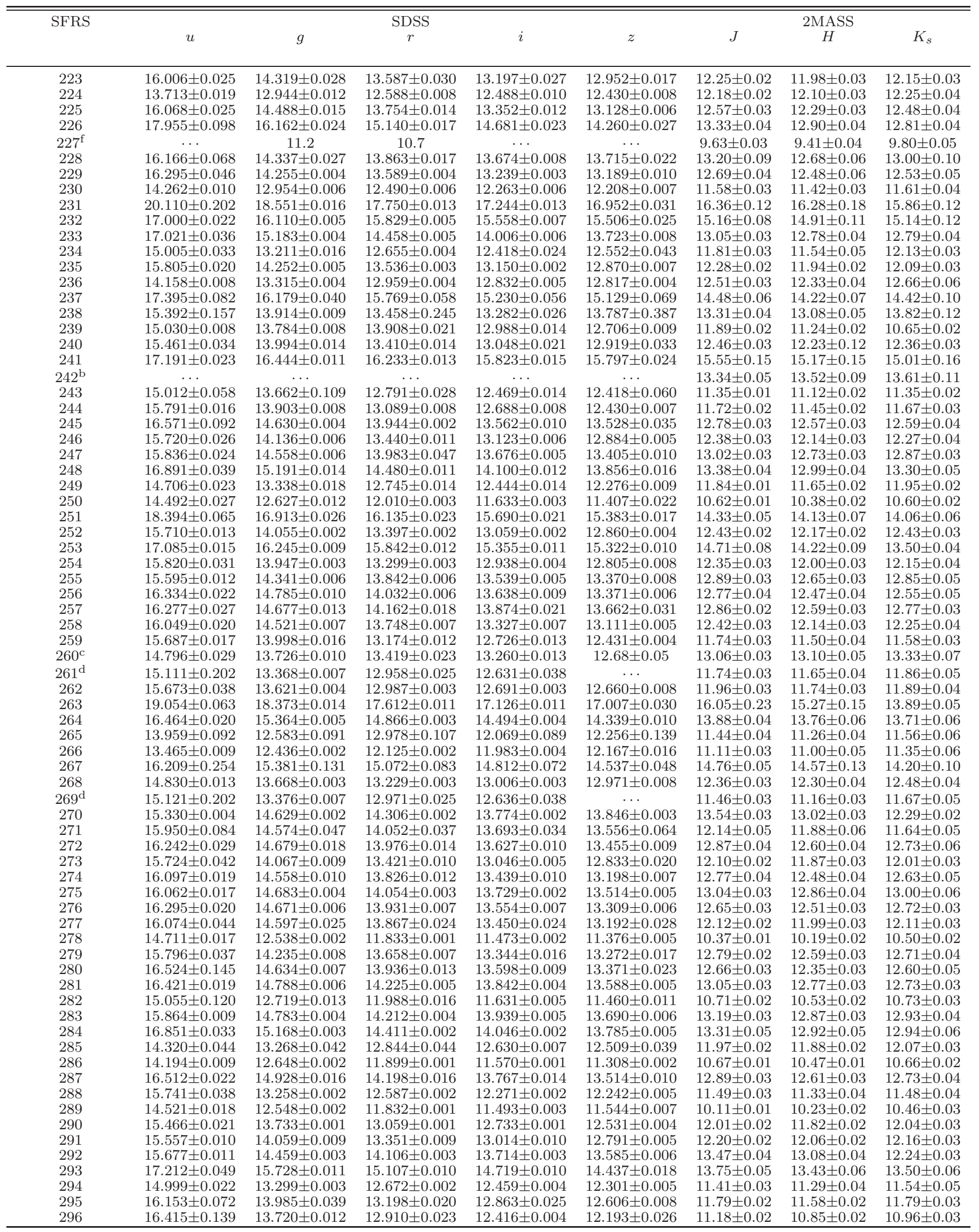


TABLE $9-$ Continued

\begin{tabular}{|c|c|c|c|c|c|c|c|c|}
\hline \multirow[t]{2}{*}{ SFRS } & \multicolumn{5}{|c|}{ SDSS } & \multicolumn{3}{|c|}{ 2MASS } \\
\hline & $u$ & $g$ & $r$ & $i$ & $z$ & $J$ & $H$ & $K_{s}$ \\
\hline 297 & $17.570 \pm 0.062$ & $15.960 \pm 0.016$ & $15.151 \pm 0.014$ & $14.700 \pm 0.014$ & \pm 0.010 & $13.84 \pm 0.04$ & $13.62 \pm 0.05$ & \pm 0.06 \\
\hline 299 & $16.764 \pm 0.051$ & $14.907 \pm 0.038$ & $14.142 \pm 0.030$ & $13.735 \pm 0.025$ & $13.517 \pm 0.023$ & $12.81 \pm 0.03$ & $12.54 \pm 0.03$ & $12.83 \pm 0.05$ \\
\hline 300 & $14.395 \pm 0.008$ & $13.238 \pm 0.055$ & $12.791 \pm 0.006$ & $12.581 \pm 0.007$ & $12.497 \pm 0.006$ & $11.10 \pm 0.02$ & $10.92 \pm 0.03$ & $11.32 \pm 0.04$ \\
\hline 301 & $14.137 \pm 0.009$ & $12.820 \pm 0.002$ & $12.266 \pm 0.006$ & $12.034 \pm 0.002$ & $11.989 \pm 0.005$ & $11.24 \pm 0.03$ & $11.09 \pm 0.03$ & $11.30 \pm 0.04$ \\
\hline 302 & $17.176 \pm 0.036$ & $15.532 \pm 0.007$ & $14.710 \pm 0.004$ & $14.311 \pm 0.004$ & $14.070 \pm 0.009$ & $13.41 \pm 0.03$ & $12.91 \pm 0.04$ & $13.11 \pm 0.04$ \\
\hline 305 & $15.337 \pm 0.013$ & $13.767 \pm 0.017$ & $13.107 \pm 0.013$ & $12.743 \pm 0.014$ & $12.489 \pm 0.004$ & $11.93 \pm 0.02$ & $11.64 \pm 0.02$ & $11.77 \pm 0.02$ \\
\hline 306 & $15.348 \pm 0.043$ & $14.078 \pm 0.012$ & $13.415 \pm 0.005$ & $13.087 \pm 0.019$ & $12.883 \pm 0.027$ & $12.28 \pm 0.02$ & $12.03 \pm 0.03$ & $12.16 \pm 0.03$ \\
\hline 307 & $15.408 \pm 0.032$ & $13.728 \pm 0.052$ & $13.049 \pm 0.068$ & $12.649 \pm 0.035$ & $12.395 \pm 0.013$ & $11.86 \pm 0.02$ & $11.62 \pm 0.03$ & $11.75 \pm 0.03$ \\
\hline 308 & $15.028 \pm 0.011$ & $13.668 \pm 0.011$ & $13.116 \pm 0.021$ & $12.802 \pm 0.023$ & $12.622 \pm 0.012$ & $12.00 \pm 0.02$ & $11.75 \pm 0.02$ & $11.86 \pm 0.02$ \\
\hline 309 & $16.234 \pm 0.091$ & $14.387 \pm 0.030$ & $13.711 \pm 0.025$ & $13.322 \pm 0.022$ & $13.203 \pm 0.021$ & $12.42 \pm 0.02$ & $12.19 \pm 0.02$ & $12.29 \pm 0.02$ \\
\hline 310 & $16.619 \pm 0.052$ & $14.900 \pm 0.011$ & $13.967 \pm 0.006$ & $13.508 \pm 0.007$ & $13.193 \pm 0.023$ & $12.41 \pm 0.02$ & $12.08 \pm 0.02$ & $12.13 \pm 0.03$ \\
\hline 311 & $16.931 \pm 0.020$ & $15.327 \pm 0.002$ & $14.579 \pm 0.002$ & $14.181 \pm 0.002$ & $13.884 \pm 0.005$ & $13.42 \pm 0.03$ & $13.08 \pm 0.04$ & $13.14 \pm 0.04$ \\
\hline 312 & $14.186 \pm 0.017$ & $12.882 \pm 0.002$ & $12.395 \pm 0.002$ & $12.153 \pm 0.002$ & $12.515 \pm 0.024$ & $11.10 \pm 0.03$ & $10.93 \pm 0.04$ & $11.34 \pm 0.05$ \\
\hline 318 & $15.741 \pm 0.030$ & $14.084 \pm 0.042$ & $13.393 \pm 0.035$ & $13.062 \pm 0.030$ & $12.859 \pm 0.016$ & $12.23 \pm 0.01$ & $12.00 \pm 0.02$ & $12.22 \pm 0.02$ \\
\hline 319 & $16.909 \pm 0.029$ & $15.502 \pm 0.006$ & $14.969 \pm 0.008$ & $14.611 \pm 0.008$ & $14.436 \pm 0.010$ & $14.01 \pm 0.05$ & $13.70 \pm 0.06$ & $13.73 \pm 0.07$ \\
\hline 320 & $16.642 \pm 0.030$ & $14.706 \pm 0.027$ & $14.069 \pm 0.029$ & $13.732 \pm 0.026$ & $13.501 \pm 0.016$ & $11.70 \pm 0.02$ & $11.41 \pm 0.02$ & $11.79 \pm 0.07$ \\
\hline 321 & $15.254 \pm 0.013$ & $14.029 \pm 0.005$ & $13.524 \pm 0.004$ & $13.244 \pm 0.003$ & $13.086 \pm 0.005$ & $12.56 \pm 0.02$ & $12.41 \pm 0.04$ & $12.53 \pm 0.03$ \\
\hline 322 & $14.271 \pm 0.003$ & $13.913 \pm 0.003$ & $13.546 \pm 0.005$ & $13.303 \pm 0.005$ & $13.121 \pm 0.005$ & $12.43 \pm 0.02$ & $12.16 \pm 0.03$ & $12.33 \pm 0.02$ \\
\hline 323 & $15.392 \pm 0.020$ & $13.615 \pm 0.003$ & $12.963 \pm 0.001$ & $12.619 \pm 0.002$ & $12.655 \pm 0.006$ & $12.11 \pm 0.03$ & $11.94 \pm 0.03$ & $12.13 \pm 0.05$ \\
\hline 324 & $14.432 \pm 0.021$ & $13.141 \pm 0.005$ & $12.645 \pm 0.005$ & $12.421 \pm 0.005$ & $12.466 \pm 0.009$ & $11.71 \pm 0.03$ & $11.50 \pm 0.03$ & $11.81 \pm 0.05$ \\
\hline 325 & $16.010 \pm 0.016$ & $14.340 \pm 0.004$ & $13.630 \pm 0.004$ & $13.269 \pm 0.004$ & $13.037 \pm 0.004$ & $12.53 \pm 0.03$ & $12.22 \pm 0.03$ & $12.35 \pm 0.04$ \\
\hline 326 & $16.893 \pm 0.038$ & $15.429 \pm 0.007$ & $14.705 \pm 0.004$ & $14.293 \pm 0.004$ & $14.014 \pm 0.009$ & $13.45 \pm 0.03$ & $13.18 \pm 0.04$ & $13.19 \pm 0.04$ \\
\hline 327 & $15.061 \pm 0.012$ & $14.695 \pm 0.017$ & $14.562 \pm 0.019$ & $14.687 \pm 0.023$ & $14.576 \pm 0.027$ & $14.47 \pm 0.06$ & $14.42 \pm 0.08$ & $14.64 \pm 0.12$ \\
\hline 328 & $15.225 \pm 0.012$ & $14.109 \pm 0.005$ & $13.625 \pm 0.006$ & $13.382 \pm 0.005$ & $13.245 \pm 0.006$ & $12.76 \pm 0.02$ & $12.54 \pm 0.03$ & $12.82 \pm 0.04$ \\
\hline 329 & $19.105 \pm 0.108$ & $17.684 \pm 0.025$ & $16.726 \pm 0.014$ & $16.256 \pm 0.015$ & $15.885 \pm 0.021$ & $15.04 \pm 0.10$ & $14.37 \pm 0.09$ & $14.25 \pm 0.07$ \\
\hline 330 & $16.116 \pm 0.868$ & $14.139 \pm 0.121$ & $13.455 \pm 0.067$ & $13.024 \pm 0.108$ & $12.515 \pm 0.354$ & $11.93 \pm 0.03$ & $11.64 \pm 0.02$ & $11.74 \pm 0.02$ \\
\hline 331 & $16.757 \pm 0.120$ & $15.037 \pm 0.149$ & $14.196 \pm 0.103$ & $13.734 \pm 0.085$ & $13.440 \pm 0.023$ & $12.50 \pm 0.03$ & $12.12 \pm 0.03$ & $12.08 \pm 0.03$ \\
\hline 332 & $15.762 \pm 0.015$ & $14.209 \pm 0.007$ & $13.560 \pm 0.006$ & $13.184 \pm 0.007$ & $13.034 \pm 0.006$ & $12.43 \pm 0.03$ & $12.16 \pm 0.03$ & $12.33 \pm 0.03$ \\
\hline 343 & $16.115 \pm 0.051$ & $14.199 \pm 0.004$ & $13.289 \pm 0.002$ & $12.831 \pm 0.002$ & $12.540 \pm 0.008$ & $11.90 \pm 0.02$ & $11.66 \pm 0.02$ & $11.79 \pm 0.03$ \\
\hline 344 & $14.925 \pm 0.030$ & $13.247 \pm 0.004$ & $12.543 \pm 0.006$ & $12.167 \pm 0.002$ & $11.980 \pm 0.008$ & $11.39 \pm 0.02$ & $11.10 \pm 0.02$ & $11.28 \pm 0.02$ \\
\hline 345 & $15.435 \pm 0.015$ & $14.263 \pm 0.022$ & $13.912 \pm 0.014$ & $13.714 \pm 0.012$ & $13.658 \pm 0.008$ & $13.08 \pm 0.04$ & $13.15 \pm 0.06$ & $13.14 \pm 0.06$ \\
\hline 346 & $14.475 \pm 0.013$ & $13.387 \pm 0.006$ & $13.009 \pm 0.005$ & $12.805 \pm 0.004$ & $12.798 \pm 0.008$ & $12.37 \pm 0.03$ & $12.17 \pm 0.04$ & $12.25 \pm 0.04$ \\
\hline 347 & $18.439 \pm 0.109$ & $17.023 \pm 0.062$ & $16.538 \pm 0.119$ & $16.089 \pm 0.108$ & $16.018 \pm 0.182$ & $14.74 \pm 0.07$ & $14.49 \pm 0.10$ & $14.22 \pm 0.07$ \\
\hline 348 & $15.263 \pm 0.005$ & $14.400 \pm 0.002$ & $14.104 \pm 0.002$ & $13.886 \pm 0.002$ & $13.735 \pm 0.004$ & $13.54 \pm 0.03$ & $13.25 \pm 0.04$ & $13.46 \pm 0.06$ \\
\hline 349 & $15.929 \pm 0.012$ & $14.386 \pm 0.007$ & $13.631 \pm 0.007$ & $13.259 \pm 0.007$ & $12.984 \pm 0.006$ & $12.22 \pm 0.03$ & $12.13 \pm 0.03$ & $12.54 \pm 0.05$ \\
\hline 350 & $15.554 \pm 0.017$ & $14.509 \pm 0.003$ & $13.960 \pm 0.003$ & $13.661 \pm 0.002$ & $13.577 \pm 0.012$ & $13.01 \pm 0.04$ & $12.88 \pm 0.05$ & $12.82 \pm 0.04$ \\
\hline $351^{f}$ & & 14.5 & 13.7 & & & $12.37 \pm 0.02$ & $12.16 \pm 0.03$ & $12.37 \pm 0.04$ \\
\hline 352 & $16.968 \pm 0.025$ & $14.977 \pm 0.003$ & $13.990 \pm 0.002$ & $13.490 \pm 0.002$ & $13.150 \pm 0.004$ & $12.77 \pm 0.03$ & $12.55 \pm 0.03$ & $12.79 \pm 0.04$ \\
\hline 353 & $14.383 \pm 0.010$ & $13.611 \pm 0.005$ & $13.424 \pm 0.004$ & $13.377 \pm 0.002$ & $13.302 \pm 0.005$ & $13.11 \pm 0.03$ & $13.06 \pm 0.04$ & $13.38 \pm 0.06$ \\
\hline 354 & $18.942 \pm 0.107$ & $17.274 \pm 0.009$ & $16.445 \pm 0.007$ & $15.912 \pm 0.008$ & $15.655 \pm 0.030$ & $14.96 \pm 0.09$ & $14.36 \pm 0.07$ & $14.48 \pm 0.09$ \\
\hline 355 & $15.102 \pm 0.873$ & $13.842 \pm 0.945$ & $13.228 \pm 0.860$ & $13.995 \pm 1.052$ & $11.806 \pm 0.559$ & $13.26 \pm 0.04$ & $13.04 \pm 0.05$ & $13.08 \pm 0.05$ \\
\hline $356^{\mathrm{g}}$ & 15.0 & 14.3 & 13.9 & & & $12.83 \pm 0.05$ & $12.68 \pm 0.06$ & $12.70 \pm 0.07$ \\
\hline 357 & $16.763 \pm 0.111$ & $15.572 \pm 0.055$ & $15.088 \pm 0.132$ & $14.732 \pm 0.053$ & $14.519 \pm 0.024$ & $14.11 \pm 0.05$ & $14.70 \pm 0.00$ & $13.95 \pm 0.07$ \\
\hline 358 & $18.800 \pm 0.118$ & $17.284 \pm 0.035$ & $16.480 \pm 0.030$ & $16.033 \pm 0.039$ & $15.635 \pm 0.034$ & $14.86 \pm 0.07$ & $14.59 \pm 0.08$ & $14.66 \pm 0.09$ \\
\hline 359 & $15.953 \pm 0.041$ & $14.427 \pm 0.005$ & $13.724 \pm 0.004$ & $13.340 \pm 0.008$ & $13.064 \pm 0.017$ & $12.50 \pm 0.03$ & $12.27 \pm 0.03$ & $12.37 \pm 0.03$ \\
\hline 360 & $15.508 \pm 0.035$ & $14.115 \pm 0.010$ & $13.465 \pm 0.010$ & $13.128 \pm 0.019$ & $12.918 \pm 0.005$ & $12.35 \pm 0.02$ & $12.14 \pm 0.02$ & $12.31 \pm 0.03$ \\
\hline 361 & $16.665 \pm 0.045$ & $14.926 \pm 0.020$ & $14.265 \pm 0.025$ & $13.875 \pm 0.021$ & $13.655 \pm 0.023$ & $13.13 \pm 0.03$ & $13.00 \pm 0.05$ & $12.98 \pm 0.05$ \\
\hline 362 & $15.972 \pm 0.068$ & $15.090 \pm 0.045$ & $14.626 \pm 0.027$ & $14.478 \pm 0.026$ & $14.477 \pm 0.036$ & $13.86 \pm 0.07$ & $13.84 \pm 0.11$ & $13.65 \pm 0.10$ \\
\hline 363 & $15.762 \pm 0.013$ & $14.398 \pm 0.002$ & $13.786 \pm 0.002$ & $13.464 \pm 0.002$ & $13.300 \pm 0.004$ & $12.85 \pm 0.03$ & $12.58 \pm 0.04$ & $12.79 \pm 0.04$ \\
\hline 364 & $15.399 \pm 0.038$ & $13.409 \pm 0.016$ & $12.631 \pm 0.027$ & $12.208 \pm 0.012$ & $12.139 \pm 0.017$ & $11.50 \pm 0.02$ & $11.22 \pm 0.02$ & $11.44 \pm 0.02$ \\
\hline 365 & $17.474 \pm 0.058$ & $15.744 \pm 0.012$ & $14.875 \pm 0.016$ & $14.429 \pm 0.008$ & $14.110 \pm 0.012$ & $13.41 \pm 0.03$ & $13.19 \pm 0.04$ & $13.31 \pm 0.04$ \\
\hline 366 & $16.263 \pm 0.049$ & $14.935 \pm 0.009$ & $14.494 \pm 0.006$ & $14.249 \pm 0.015$ & $14.410 \pm 0.072$ & $13.54 \pm 0.04$ & $13.31 \pm 0.06$ & $13.45 \pm 0.06$ \\
\hline 367 & $17.624 \pm 0.060$ & $16.000 \pm 0.030$ & $15.272 \pm 0.029$ & $14.883 \pm 0.030$ & $14.601 \pm 0.016$ & $14.02 \pm 0.04$ & $13.84 \pm 0.05$ & $13.96 \pm 0.07$ \\
\hline 368 & $16.678 \pm 0.028$ & $15.129 \pm 0.003$ & $14.363 \pm 0.002$ & $13.947 \pm 0.002$ & $13.665 \pm 0.009$ & $13.13 \pm 0.03$ & $12.83 \pm 0.03$ & $12.94 \pm 0.04$ \\
\hline 369 & $17.781 \pm 0.028$ & $16.217 \pm 0.005$ & $15.440 \pm 0.004$ & $14.926 \pm 0.004$ & $14.662 \pm 0.008$ & $14.03 \pm 0.03$ & $13.72 \pm 0.04$ & $13.64 \pm 0.03$ \\
\hline
\end{tabular}


Ashby et al.

TABLE 9 - Continued

\begin{tabular}{|c|c|c|c|c|c|c|c|c|}
\hline \multirow{2}{*}{ SFRS } & \multicolumn{5}{|c|}{ SDSS } & \multicolumn{3}{|c|}{ 2MASS } \\
\hline & $u$ & $g$ & $r$ & $i$ & $z$ & $J$ & $H$ & $K_{s}$ \\
\hline
\end{tabular}

Note. - SDSS DR7 Petrosian magnitudes and 2MASS total magnitudes for the SFRS galaxies. Where the SDSS photometry is unreliable due to shredding, no values are given. Uncertainties are taken from the 2MASS extended source catalog and reflect measurement errors only. Issues pertaining to systematic errors in the 2MASS data are discussed in Sec. 4.3.2 Uncertainties are taken from the 2MASS extended source catalog and reflect measurement errors only. Issues pertaining to systematic errors in the 2MASS data are discussed in Sec. 4.3.2 All magnitudes are expressed on the AB system.

${ }^{\mathrm{a}}$ SDSS $u$ photometry was replaced by an estimate based on applying the Jester et al. (2005) filter transformations to the global $B V R I$ photometry from Hyperleda.

b Available SDSS ugriz photometry corresponds only to a portion of this source.

${ }^{\mathrm{c}}$ SDSS $z$ photometry was replaced by an estimate based on applying the Jester et al. (2005) filter transformations to the global $B V R I$ photometry from Hyperleda.

d Available SDSS $z$ photometry corresponds only to a portion of this source.

e SDSS ugriz photometry was replaced by an estimate based on applying the Jester et al. (2005) filter transformations to the global BVRI photometry from Hyperleda.

${ }^{\mathrm{f}}$ SDSS gr photometry was replaced by an estimate based on applying the Jester et al. (2005) filter transformations to the global $B V R I$ photometry from Hyperleda.

g SDSS ugr photometry was replaced by an estimate based on applying the Jester et al. (2005) filter transformations to the global $B V R I$ photometry from Hyperleda. 